\title{
Changes in Climate Extremes and their Impacts on the Natural Physical Environment
}

\author{
Coordinating Lead Authors: \\ Sonia I. Seneviratne (Switzerland), Neville Nicholls (Australia)
}

\section{Lead Authors:}

David Easterling (USA), Clare M. Goodess (United Kingdom), Shinjiro Kanae (Japan), James Kossin (USA), Yali Luo (China), Jose Marengo (Brazil), Kathleen Mclnnes (Australia), Mohammad Rahimi (Iran), Markus Reichstein (Germany), Asgeir Sorteberg (Norway), Carolina Vera (Argentina), Xuebin Zhang (Canada)

\section{Review Editors:}

Matilde Rusticucci (Argentina), Vladimir Semenov (Russia)

\section{Contributing Authors:}

Lisa V. Alexander (Australia), Simon Allen (Switzerland), Gerardo Benito (Spain), Tereza Cavazos (Mexico), John Clague (Canada), Declan Conway (United Kingdom), Paul M. Della-Marta (Switzerland), Markus Gerber (Switzerland), Sunling Gong (Canada), B. N. Goswami (India), Mark Hemer (Australia), Christian Huggel (Switzerland), Bart van den Hurk (Netherlands), Viatcheslav V. Kharin (Canada), Akio Kitoh (Japan), Albert M.G. Klein Tank (Netherlands), Guilong Li (Canada), Simon Mason (USA), William McGuire (United Kingdom), Geert Jan van Oldenborgh (Netherlands), Boris Orlowsky (Switzerland), Sharon Smith (Canada), Wassila Thiaw (USA), Adonis Velegrakis (Greece), Pascal Yiou (France), Tingjun Zhang (USA), Tianjun Zhou (China), Francis W. Zwiers (Canada)

This chapter should be cited as:

Seneviratne, S.I., N. Nicholls, D. Easterling, C.M. Goodess, S. Kanae, J. Kossin, Y. Luo, J. Marengo, K. McInnes, M. Rahimi, M. Reichstein, A. Sorteberg, C. Vera, and X. Zhang, 2012: Changes in climate extremes and their impacts on the natural physical environment. In: Managing the Risks of Extreme Events and Disasters to Advance Climate Change Adaptation [Field, C.B., V. Barros, T.F. Stocker, D. Qin, D.J. Dokken, K.L. Ebi, M.D. Mastrandrea, K.J. Mach, G.-K. Plattner, S.K. Allen, M. Tignor, and P.M. Midgley (eds.)]. A Special Report of Working Groups I and II of the Intergovernmental Panel on Climate Change (IPCC). Cambridge University Press, Cambridge, UK, and New York, NY, USA, pp. 109-230. 


\section{Table of Contents}

Executive Summary

3.1. Weather and Climate Events Related to Disasters ..........................................................115

3.1.1. Categories of Weather and Climate Events Discussed in this Chapter .................................................................115

3.1.2. Characteristics of Weather and Climate Events Relevant to Disasters .................................................................115

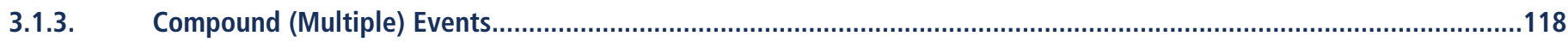

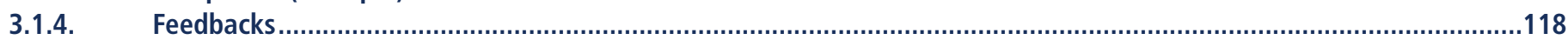

3.1.5. Confidence and Likelihood of Assessed Changes in Extremes ............................................................................120

3.1.6. Changes in Extremes and Their Relationship to Changes in Regional and Global Mean Climate...............................121

3.1.7. Surprises / Abrupt Climate Change .........................................................................................................................122

3.2. Requirements and Methods for Analyzing Changes in Extremes......................................122

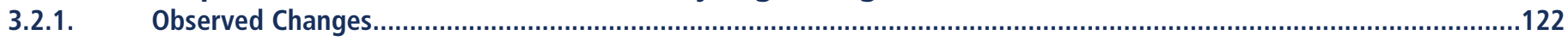

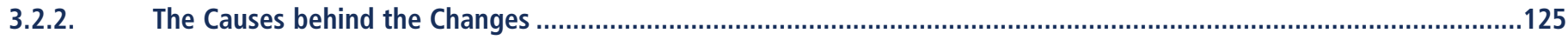

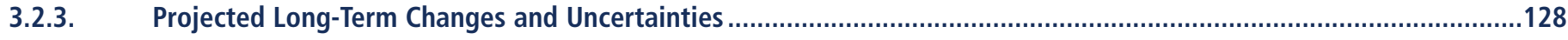

3.3. Observed and Projected Changes in Weather and Climate Extremes .................................133

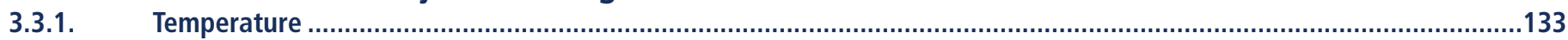

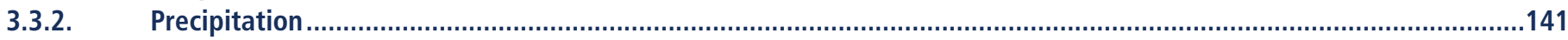

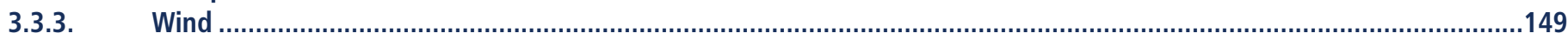

3.4. Observed and Projected Changes in Phenomena Related to Weather and Climate Extremes ...................................................152

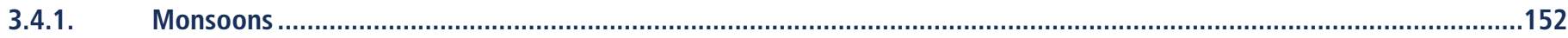

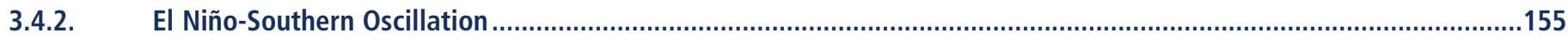

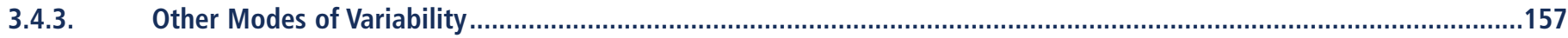

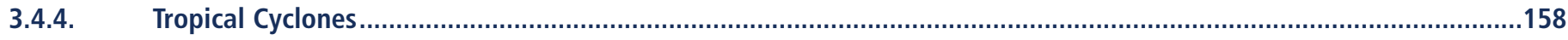

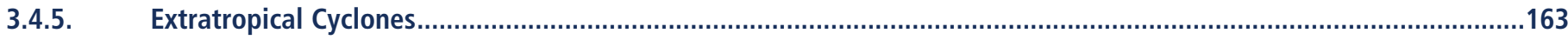

3.5. Observed and Projected Impacts on the Natural Physical Environment..............................167

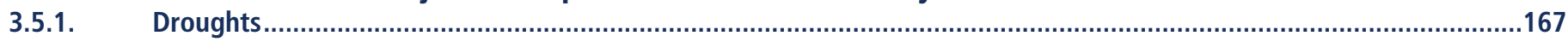

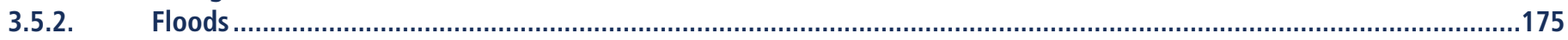

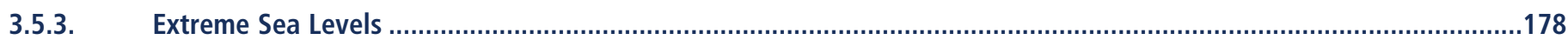

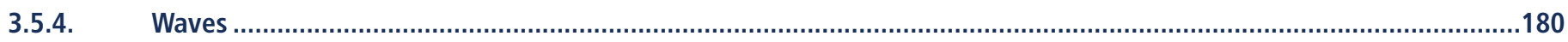

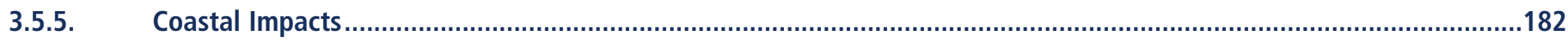

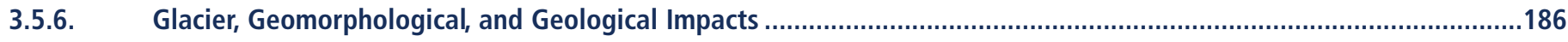

3.5.7. High-latitude Changes Including Permafrost.......................................................................................................189

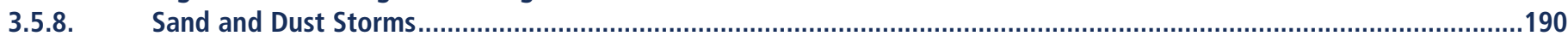

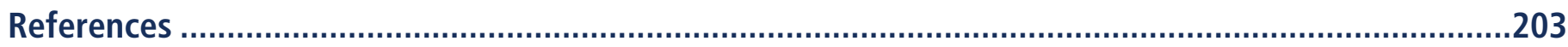

Boxes and Frequently Asked Questions

Box 3-1. Definition and Analysis of Climate Extremes in the Scientific Literature ...................................................................116

FAQ 3.1. Is the Climate Becoming More Extreme? …………………………………………………………………..124

FAQ 3.2. Has Climate Change Affected Individual Extreme Events? ……………............................................................127

Box 3-2. Variations in Confidence in Projections of Climate Change: Mean versus Extremes, Variables, Scale ..........................132

Box 3-3. The Definition of Drought........................................................................................................................................167

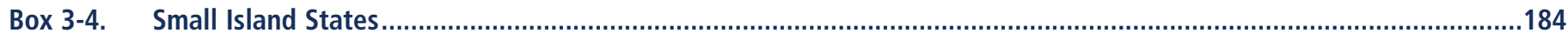




\section{Executive Summary}

This chapter addresses changes in weather and climate events relevant to extreme impacts and disasters. An extreme (weather or climate) event is generally defined as the occurrence of a value of a weather or climate variable above (or below) a threshold value near the upper (or lower) ends ('tails') of the range of observed values of the variable. Some climate extremes (e.g., droughts, floods) may be the result of an accumulation of weather or climate events that are, individually, not extreme themselves (though their accumulation is extreme). As well, weather or climate events, even if not extreme in a statistical sense, can still lead to extreme conditions or impacts, either by crossing a critical threshold in a social, ecological, or physical system, or by occurring simultaneously with other events. A weather system such as a tropical cyclone can have an extreme impact, depending on where and when it approaches landfall, even if the specific cyclone is not extreme relative to other tropical cyclones. Conversely, not all extremes necessarily lead to serious impacts. [3.1]

Many weather and climate extremes are the result of natural climate variability (including phenomena such as El Niño), and natural decadal or multi-decadal variations in the climate provide the backdrop for anthropogenic climate changes. Even if there were no anthropogenic changes in climate, a wide variety of natural weather and climate extremes would still occur. [3.1]

A changing climate leads to changes in the frequency, intensity, spatial extent, duration, and timing of weather and climate extremes, and can result in unprecedented extremes. Changes in extremes can also be directly related to changes in mean climate, because mean future conditions in some variables are projected to lie within the tails of present-day conditions. Nevertheless, changes in extremes of a climate or weather variable are not always related in a simple way to changes in the mean of the same variable, and in some cases can be of opposite sign to a change in the mean of the variable. Changes in phenomena such as the El Niño-Southern Oscillation or monsoons could affect the frequency and intensity of extremes in several regions simultaneously. [3.1]

Many factors affect confidence in observed and projected changes in extremes. Our confidence in observed changes in extremes depends on the quality and quantity of available data and the availability of studies analyzing these data. It consequently varies between regions and for different extremes. Similarly, our confidence in projecting changes (including the direction and magnitude of changes in extremes) varies with the type of extreme, as well as the considered region and season, depending on the amount and quality of relevant observational data and model projections, the level of understanding of the underlying processes, and the reliability of their simulation in models (assessed from expert judgment, model validation, and model agreement). Global-scale trends in a specific extreme may be either more reliable (e.g., for temperature extremes) or less reliable (e.g., for droughts) than some regionalscale trends, depending on the geographical uniformity of the trends in the specific extreme. 'Low confidence' in observed or projected changes in a specific extreme neither implies nor excludes the possibility of changes in this extreme. [3.1.5, 3.1.6, 3.2.3; Box 3-2; Figures 3-3, 3-4, 3-5, 3-6, 3-7, 3-8, 3-10]

There is evidence from observations gathered since 1950 of change in some extremes. It is very likely that there has been an overall decrease in the number of cold days and nights, and an overall increase in the number of warm days and nights, at the global scale, that is, for most land areas with sufficient data. It is likely that these changes have also occurred at the continental scale in North America, Europe, and Australia. There is medium confidence of a warming trend in daily temperature extremes in much of Asia. Confidence in observed trends in daily temperature extremes in Africa and South America generally varies from low to medium depending on the region. Globally, in many (but not all) regions with sufficient data there is medium confidence that the length or number of warm spells or heat waves has increased since the middle of the 20th century. It is likely that there have been statistically significant increases in the number of heavy precipitation events (e.g., 95th percentile) in more regions than there have been statistically significant decreases, but there are strong regional and subregional variations in the trends. There is low confidence that any observed long-term (i.e., 40 years or more) increases in tropical cyclone activity are robust, after accounting for past changes in observing capabilities. It is likely that there has been a poleward shift in the main Northern and Southern Hemisphere extratropical storm tracks. There is low confidence in observed trends in 
small-scale phenomena such as tornadoes and hail because of data inhomogeneities and inadequacies in monitoring systems. There is medium confidence that since the 1950s some regions of the world have experienced a trend to more intense and longer droughts, in particular in southern Europe and West Africa, but in some regions droughts have become less frequent, less intense, or shorter, for example, in central North America and northwestern Australia. There is limited to medium evidence available to assess climate-driven observed changes in the magnitude and frequency of floods at regional scales because the available instrumental records of floods at gauge stations are limited in space and time, and because of confounding effects of changes in land use and engineering. Furthermore, there is low agreement in this evidence, and thus overall low confidence at the global scale regarding even the sign of these changes. It is likely that there has been an increase in extreme coastal high water related to increases in mean sea level in the late 20th century. [3.2.1, 3.3.1, 3.3.2, 3.3.3, 3.4.4, 3.4.5, 3.5.1, 3.5.2, 3.5.3; Tables 3-1, 3-2]

There is evidence that some extremes have changed as a result of anthropogenic influences, including increases in atmospheric concentrations of greenhouse gases. It is likely that anthropogenic influences have led to warming of extreme daily minimum and maximum temperatures at the global scale. There is medium confidence that anthropogenic influences have contributed to intensification of extreme precipitation at the global scale. It is likely that there has been an anthropogenic influence on increasing extreme coastal high water due to an increase in mean sea level. The uncertainties in the historical tropical cyclone records, the incomplete understanding of the physical mechanisms linking tropical cyclone metrics to climate change, and the degree of tropical cyclone variability provide only low confidence for the attribution of any detectable changes in tropical cyclone activity to anthropogenic influences. Attribution of single extreme events to anthropogenic climate change is challenging. [3.2.2, 3.3.1, 3.3.2, 3.4.4, 3.5.3; Table 3-1]

The following assessments of the likelihood of and/or confidence in projections are generally for the end of the 21st century and relative to the climate at the end of the 20th century. There are three main sources of uncertainty in the projections: the natural variability of climate; uncertainties in climate model parameters and structure; and projections of future emissions. Projections for differing emissions scenarios generally do not strongly diverge in the coming two to three decades, but uncertainty in the sign of change is relatively large over this time frame because climate change signals are expected to be relatively small compared to natural climate variability. For certain extremes (e.g., precipitation-related extremes), the uncertainty in projected changes by the end of the 21st century is more the result of uncertainties in climate models rather than uncertainties in future emissions. For other extremes (in particular temperature extremes at the global scale and in most regions), the emissions uncertainties are the main source of uncertainty in projections for the end of the 21st century. In the assessments provided in this chapter, uncertainties in projections from the direct evaluation of multi-model ensemble projections are modified by taking into account the past performance of models in simulating extremes (for instance, simulations of late 20thcentury changes in extreme temperatures appear to overestimate the observed warming of warm extremes and underestimate the warming of cold extremes), the possibility that some important processes relevant to extremes may be missing or be poorly represented in models, and the limited number of model projections and corresponding analyses currently available of extremes. For these reasons the assessed uncertainty is generally greater than would be assessed from the model projections alone. Low-probability, high-impact changes associated with the crossing of poorly understood climate thresholds cannot be excluded, given the transient and complex nature of the climate system. Feedbacks play an important role in either damping or enhancing extremes in several climate variables. $[3.1 .4,3.1 .7,3.2 .3,3.3 .1,3.3 .2 ;$ Box 3-2]

Models project substantial warming in temperature extremes by the end of the 21 st century. It is virtually certain that increases in the frequency and magnitude of warm daily temperature extremes and decreases in cold extremes will occur through the 21st century at the global scale. It is very likely that the length, frequency, and/or intensity of warm spells or heat waves will increase over most land areas. For the Special Report on Emissions Scenarios (SRES) A2 and A1B emission scenarios, a 1-in-20 year annual hottest day is likely to become a 1-in-2 year annual extreme by the end of the 21st century in most regions, except in the high latitudes of the Northern Hemisphere where it is likely to become a 1 -in-5 year annual extreme. In terms of absolute values, 20-year extreme annual daily maximum temperature (i.e., return value) will likely increase by about 1 to $3^{\circ} \mathrm{C}$ by mid-21st century and by about 2 to $5^{\circ} \mathrm{C}$ by the late 21 st century, depending on the region and emissions scenario (considering the $\mathrm{B} 1, \mathrm{~A} 1 \mathrm{~B}$, 
and A2 scenarios). Regional changes in temperature extremes will often differ from the mean global temperature change. [3.3.1; Table 3-3; Figure 3-5]

It is likely that the frequency of heavy precipitation or the proportion of total rainfall from heavy rainfalls will increase in the 21st century over many areas of the globe. This is particularly the case in the high latitudes and tropical regions, and in winter in the northern mid-latitudes. Heavy rainfalls associated with tropical cyclones are likely to increase with continued warming induced by enhanced greenhouse gas concentrations. There is medium confidence that, in some regions, increases in heavy precipitation will occur despite projected decreases in total precipitation. For a range of emission scenarios (SRES A2, A1B, and B1), a 1-in-20 year annual maximum 24-hour precipitation rate is likely to become a 1 -in-5 to 1 -in-15 year event by the end of the 21 st century in many regions, and in most regions the higher emissions scenarios (A1B and A2) lead to a greater projected decrease in return period. Nevertheless, increases or statistically non-significant changes in return periods are projected in some regions. [3.3.2; Table 3-3; Figure 3-7]

There is generally low confidence in projections of changes in extreme winds because of the relatively few studies of projected extreme winds, and shortcomings in the simulation of these events. An exception is mean tropical cyclone maximum wind speed, which is likely to increase, although increases may not occur in all ocean basins. It is likely that the global frequency of tropical cyclones will either decrease or remain essentially unchanged. There is low confidence in projections of small-scale phenomena such as tornadoes because competing physical processes may affect future trends and because climate models do not simulate such phenomena. There is medium confidence that there will be a reduction in the number of mid-latitude cyclones averaged over each hemisphere due to future anthropogenic climate change. There is low confidence in the detailed geographical projections of mid-latitude cyclone activity. There is medium confidence in a projected poleward shift of mid-latitude storm tracks due to future anthropogenic forcings. [3.3.3, 3.4.4, 3.4.5]

Uncertainty in projections of changes in large-scale patterns of natural climate variability remains large. There is low confidence in projections of changes in monsoons (rainfall, circulation), because there is little consensus in climate models regarding the sign of future change in the monsoons. Model projections of changes in El NiñoSouthern Oscillation variability and the frequency of El Niño episodes as a consequence of increased greenhouse gas concentrations are not consistent, and so there is low confidence in projections of changes in the phenomenon. However, most models project an increase in the relative frequency of central equatorial Pacific events (which typically exhibit different patterns of climate variations than do the classical East Pacific events). There is low confidence in the ability to project changes in other natural climate modes including the North Atlantic Oscillation, the Southern Annular Mode, and the Indian Ocean Dipole. [3.4.1, 3.4.2, 3.4.3]

It is very likely that mean sea level rise will contribute to upward trends in extreme coastal high water levels in the future. There is high confidence that locations currently experiencing adverse impacts such as coastal erosion and inundation will continue to do so in the future due to increasing sea levels, all other contributing factors being equal. There is low confidence in wave height projections because of the small number of studies, the lack of consistency of the wind projections between models, and limitations in the models' ability to simulate extreme winds. Future negative or positive changes in significant wave height are likely to reflect future changes in storminess and associated patterns of wind change. [3.5.3, 3.5.4, 3.5.5]

Projected precipitation and temperature changes imply possible changes in floods, although overall there is low confidence in projections of changes in fluvial floods. Confidence is low due to limited evidence and because the causes of regional changes are complex, although there are exceptions to this statement. There is medium confidence (based on physical reasoning) that projected increases in heavy rainfall would contribute to increases in local flooding, in some catchments or regions. Earlier spring peak flows in snowmelt and glacier-fed rivers are very likely. [3.5.2]

There is medium confidence that droughts will intensify in the 21st century in some seasons and areas, due to reduced precipitation and/or increased evapotranspiration. This applies to regions including southern 
Europe and the Mediterranean region, central Europe, central North America, Central America and Mexico, northeast Brazil, and southern Africa. Definitional issues, lack of observational data, and the inability of models to include all the factors that influence droughts preclude stronger confidence than medium in the projections. Elsewhere there is overall low confidence because of inconsistent projections of drought changes (dependent both on model and dryness index). There is low confidence in projected future changes in dust storms although an increase could be expected where aridity increases. [3.5.1, 3.5.8; Box 3-3; Table 3-3; Figure 3-10]

There is high confidence that changes in heat waves, glacial retreat, and/or permafrost degradation will affect high-mountain phenomena such as slope instabilities, mass movements, and glacial lake outburst floods. There is also high confidence that changes in heavy precipitation will affect landslides in some regions. There is low confidence regarding future locations and timing of large rock avalanches, as these depend on local geological conditions and other non-climatic factors. There is low confidence in projections of an anthropogenic effect on phenomena such as shallow landslides in temperate and tropical regions, because these are strongly influenced by human activities such as land use practices, deforestation, and overgrazing. [3.5.6, 3.5.7]

The small land area and often low elevation of small island states make them particularly vulnerable to rising sea levels and impacts such as inundation, shoreline change, and saltwater intrusion into underground aquifers. Short record lengths and the inadequate resolution of current climate models to represent small island states limit the assessment of changes in extremes. There is insufficient evidence to assess observed trends and future projections in rainfall across the small island regions considered here. There is medium confidence in projected temperature increases across the Caribbean. The very likely contribution of mean sea level rise to increased extreme coastal high water levels, coupled with the likely increase in tropical cyclone maximum wind speed, is a specific issue for tropical small island states. [3.4.4, 3.5.3; Box 3-4]

This chapter does not provide assessments of projected changes in extremes at spatial scales smaller than for large regions. These large-region projections provide a wider context for national or local projections, where these exist, and where they do not exist, a first indication of expected changes, their associated uncertainties, and the evidence available. [3.2.3.1] 


\subsection{Weather and Climate Events Related to Disasters}

A changing climate leads to changes in the frequency, intensity, spatial extent, duration, and timing of weather and climate extremes, and can result in unprecedented extremes (Sections 3.1.7, 3.3, 3.4, and 3.5). As well, weather or climate events, even if not extreme in a statistical sense, can still lead to extreme conditions or impacts, either by crossing a critical threshold in a social, ecological, or physical system, or by occurring simultaneously with other events (Sections 3.1.2, 3.1.3, 3.1.4, 3.3, 3.4, and 3.5). Some climate extremes (e.g., droughts, floods) may be the result of an accumulation of weather or climate events that are, individually, not extreme themselves (though their accumulation is extreme, e.g., Section 3.1.2). A weather system such as a tropical cyclone can have an extreme impact, depending on where and when it approaches landfall, even if the specific cyclone is not extreme relative to other tropical cyclones. Conversely, not all extremes necessarily lead to serious impacts. Changes in extremes can also be directly related to changes in mean climate, because mean future conditions in some variables are projected to lie within the tails of present-day conditions (Section 3.1.6). Hence, the definition of extreme weather and climate events is complex (Section 3.1.2 and Box 3-1) and the assessment of changes in climate that are relevant to extreme impacts and disasters needs to consider several aspects. Those related to vulnerability and exposure are addressed in Chapters 2 and 4 of this report, while we focus here on the physical dimension of these events.

Many weather and climate extremes are the result of natural climate variability (including phenomena such as El Niño), and natural decadal or multi-decadal variations in the climate provide the backdrop for anthropogenic climate changes. Even if there were no anthropogenic changes in climate, a wide variety of natural weather and climate extremes would still occur.

\subsubsection{Categories of Weather and Climate Events Discussed in this Chapter}

This chapter addresses changes in weather and climate events relevant to extreme impacts and disasters grouped into the following categories:

1) Extremes of atmospheric weather and climate variables (temperature, precipitation, wind)

2) Weather and climate phenomena that influence the occurrence of extremes in weather or climate variables or are extremes themselves (monsoons, El Niño and other modes of variability, tropical and extratropical cyclones)

3) Impacts on the natural physical environment (droughts, floods, extreme sea level, waves, and coastal impacts, as well as other physical impacts, including cryosphere-related impacts, landslides, and sand and dust storms).

The distinction between these three categories is somewhat arbitrary, and the categories are also related. In the case of the third category, 'impacts on the natural physical environment,' a specific distinction between these events and those considered under 'extremes of atmospheric weather and climate variables' is that they are not caused by variations in a single atmospheric weather and climate variable, but are generally the result of specific conditions in several variables, as well as of some surface properties or states. For instance, both floods and droughts are related to precipitation extremes, but are also impacted by other atmospheric and surface conditions (and are thus often better viewed as compound events, see Section 3.1.3). Most of the impacts on the natural physical environment discussed in the third category are extremes themselves, as well as often being caused or affected by atmospheric weather or climate extremes. Another arbitrary choice made here is the separate category for phenomena (or climate or weather systems) that are related to weather and climate extremes, such as monsoons, El Niño, and other modes of variability. These phenomena affect the large-scale environment that, in turn, influences extremes. For instance, El Niño episodes typically lead to droughts in some regions with, simultaneously, heavy rains and floods occurring elsewhere. This means that all occurrences of El Niño are relevant to extremes and not only extreme El Niño episodes. A change in the frequency or nature of El Niño episodes (or in their relationships with climate in specific regions) would affect extremes in many locations simultaneously. Similarly, changes in monsoon patterns could affect several countries simultaneously. This is especially important from an international disaster perspective because coping with disasters in several regions simultaneously may be challenging (see also Section 3.1.3 and Chapters 7 and 8).

This section provides background material on the characterization and definition of extreme events, the definition and analysis of compound events, the relevance of feedbacks for extremes, the approach used for the assignment of confidence and likelihood assessments in this chapter, and the possibility of 'surprises' regarding future changes in extremes. Requirements and methods for analyzing changes in climate extremes are addressed in Section 3.2. Assessments regarding changes in the climate variables, phenomena, and impacts considered in this chapter are provided in Sections 3.3 to 3.5. Table 3-1 provides summaries of these assessments for changes at the global scale. Tables 3-2 and 3-3 (found on pages 191-202) provide more regional detail on observed and projected changes in temperature extremes, heavy precipitation, and dryness (with regions as defined in Figure 3-1). Note that impacts on ecosystems (e.g., bushfires) and human systems (e.g., urban flooding) are addressed in Chapter 4.

\subsubsection{Characteristics of Weather and Climate Events Relevant to Disasters}

The identification and definition of weather and climate events that are relevant from a risk management perspective are complex and depend on the stakeholders involved (Chapters 1 and 2). In this chapter, we focus on the assessment of changes in 'extreme climate or weather events' (also referred to herein as 'climate extremes' see below and Glossary), which generally correspond to the 'hazards' discussed in Chapter 1. 


\section{Box 3-1 | Definition and Analysis of Climate Extremes in the Scientific Literature}

This box provides some details on the definition of climate extremes in the scientific literature and on common approaches employed for their investigation.

A large amount of the available scientific literature on climate extremes is based on the use of so-called 'extreme indices,' which can either be based on the probability of occurrence of given quantities or on threshold exceedances (Section 3.1.2). Typical indices that are seen in the scientific literature include the number, percentage, or fraction of days with maximum temperature (Tmax) or minimum temperature (Tmin), below the 1st, 5th, or 10th percentile, or above the 90th, 95th, or 99th percentile, generally defined for given time frames (days, month, season, annual) with respect to the 1961-1990 reference time period. Commonly, indices for 10th and 90th percentiles of Tmax/Tmin computed on daily time frames are referred to as 'cold/warm days/nights' (e.g., Figures 3-3 and 3-4; Tables 3-1 to 3-3, and Section 3.3.1; see also Glossary). Other definitions relate to, for example, the number of days above specific absolute temperature or precipitation thresholds, or more complex definitions related to the length or persistence of climate extremes. Some advantages of using predefined extreme indices are that they allow some comparability across modelling and observational studies and across regions (although with limitations noted below). Moreover, in the case of observations, derived indices may be easier to obtain than is the case with daily temperature and precipitation data, which are not always distributed by meteorological services. Peterson and Manton (2008) discuss collaborative international efforts to monitor extremes by employing extreme indices. Typically, although not exclusively, extreme indices used in the scientific literature reflect 'moderate extremes,' for example, events occurring as often as 5 or $10 \%$ of the time. More extreme 'extremes' are often investigated using Extreme Value Theory (EVT) due to sampling issues (see below). Extreme indices are often defined for daily temperature and precipitation characteristics, and are also sometimes applied to seasonal characteristics of these variables, to other weather and climate variables, such as wind speed, humidity, or to physical impacts and phenomena. Beside analyses for temperature and precipitation indices (see Sections 3.3.1 and 3.3.2; Tables 3-2 and 3-3), other studies are, for instance, available in the literature for wind-based (Della-Marta et al., 2009) and pressure-based (Beniston, 2009a) indices, for health-relevant indices (e.g., 'heat index') combining temperature and relative humidity characteristics (e.g., Diffenbaugh et al., 2007; Fischer and Schär, 2010; Sherwood and Huber, 2010), and for a range of dryness indices (see Box 3-3).

Extreme Value Theory is an approach used for the estimation of extreme values (e.g., Coles, 2001), which aims at deriving a probability distribution of events from the tail of a probability distribution, that is, at the far end of the upper or lower ranges of the probability distributions (typically occurring less frequently than once per year or per period of interest, i.e., generally less than 1 to $5 \%$ of the considered overall sample). EVT is used to derive a complete probability distribution for such low-probability events, which can also help analyzing the probability of occurrence of events that are outside of the observed data range (with limitations). Two different approaches can be used to estimate the parameters for such probability distributions. In the block maximum approach, the probability distribution parameters are estimated for maximum values of consecutive blocks of a time series (e.g., years). In the second approach, instead of the block maxima the estimation is based on events that exceed a high threshold (peaks over threshold approach). Both approaches are used in climate research.

Hence, the present chapter does not directly consider the dimensions of vulnerability or exposure, which are critical in determining the human and ecosystem impacts of climate extremes (Chapters 1, 2, and 4).

This report defines an 'extreme climate or weather event' or 'climate extreme' as "the occurrence of a value of a weather or climate variable above (or below) a threshold value near the upper (or lower) ends of the range of observed values of the variable" (see Glossary). Several aspects of this definition can be clarified thus:

- Definitions of thresholds vary, but values with less than $10,5,1 \%$, or even lower chance of occurrence for a given time of the year (day, month, season, whole year) during a specified reference period (generally 1961-1990) are often used. In some circumstances, information from sources other than observations, such as model projections, can be used as a reference.
- Absolute thresholds (rather than these relative thresholds based on the range of observed values of a variable) can also be used to identify extreme events (e.g., specific critical temperatures for health impacts).

- What is called an extreme weather or climate event will vary from place to place in an absolute sense (e.g., a hot day in the tropics will be a different temperature than a hot day in the mid-latitudes), and possibly in time given some adaptation from society (see Box 3-1).

- Some climate extremes (e.g., droughts, floods) may be the result of an accumulation of moderate weather or climate events (this accumulation being itself extreme). Compound events (see Section 3.1.3), that is, two or more events occurring simultaneously, can lead to high impacts, even if the two single events are not extreme per se (only their combination). 
Recent publications have used other approaches for evaluating characteristics of extremes or changes in extremes, for instance, analyzing trends in record events or investigating whether records in observed time series are being set more or less frequently than would be expected in an unperturbed climate (Benestad, 2003, 2006; Zorita et al., 2008; Meehl et al., 2009c; Trewin and Vermont, 2010). Furthermore, besides the actual magnitude of extremes (quantified in terms of probability/return frequency or absolute threshold), other relevant aspects for the definition of climate extremes from an impact perspective include the event's duration, the spatial area affected, timing, frequency, onset date, continuity (i.e., whether there are 'breaks' within a spell), and preconditioning (e.g., rapid transition from a slowly developing meteorological drought into an agricultural drought, see Box 3-3). These aspects, together with seasonal variations in climate extremes, are not as frequently examined in climate models or observational analyses, and thus can only be partly assessed within this chapter.

As noted in the discussion of 'extreme weather or climate events' in Section 3.1.2, thresholds, percentiles, or return values used for the definition of climate extremes are generally defined with respect to a given reference period (generally historical, i.e., 1961-1990, but possibly also based on climate model data). In some cases, a transient baseline can also be considered (i.e., the baseline uses data from the period under examination and changes as the period being considered changes, rather than using a standard period such as 1961-1990). The choice of the reference period may be relevant for the magnitude of the assessed changes as highlighted, for example, in Lorenz et al. (2010). The choice of the reference period (static or transient) could also affect the assessment of the respective role of changes in mean versus changes in variability for changes in extremes discussed in Section 3.1.6. If extremes are based on the probability distribution from which they are drawn, then a simple change in the mean (and keeping the same distribution) would, strictly speaking, produce no relative change in extremes at all. The question of the choice of an appropriate reference period is tied to the notion of adaptation. Events that are considered extreme nowadays in some regions could possibly be adapted to if the vulnerability and exposure to these extremes is reduced (Chapters 1,2, and 4 through 7). However, there are also some limits to adaptation as highlighted in Chapter 8. These considerations are difficult to include in the statistical analyses of climate scenarios because of the number of (mostly non-physical) aspects that would need to be taken into account.

To conclude, there is no precise definition of an extreme (e.g., D.B. Stephenson et al., 2008). In particular, we note limitations in the definition of both probability-based or threshold-based climate extremes and their relations to impacts, which apply independently of the chosen method of analysis:

- An event from the extreme tails of probability distributions is not necessarily extreme in terms of impact.

- Impact-related thresholds can vary in space and time, that is, single absolute thresholds (e.g., a daily rainfall exceeding $25 \mathrm{~mm}$ or the number of frost days) will not reflect extremes in all locations and time periods (e.g., season, decade).

As an illustration, projected patterns (in the magnitude but not the sign) of changes in annual heat wave length were shown to be highly dependent on the choice of index used for the assessment of heat wave or warm spell duration (using the mean and maximum Heat Wave Duration Indices, HWDImean and HWDImax, and the Warm Spell Duration Index, WSDI; see Orlowsky and Seneviratne, 2011), because of large geographical variations in the variability of daily temperature (Alexander et al., 2006). Similar definition issues apply to other types of extremes, especially those characterizing dryness (see Section 3.5.1 and Box 3-3).

- Not all extreme weather and climate events necessarily have extreme impacts.

- The distinction between extreme weather events and extreme climate events is not precise, but is related to their specific time scales:

- An extreme weather event is typically associated with changing weather patterns, that is, within time frames of less than a day to a few weeks.

- An extreme climate event happens on longer time scales. It can be the accumulation of several (extreme or non-extreme) weather events (e.g., the accumulation of moderately belowaverage rainy days over a season leading to substantially belowaverage cumulated rainfall and drought conditions).

For simplicity, we collectively refer to both extreme weather events and extreme climate events with the term 'climate extremes' in this chapter.
From this definition, it can be seen that climate extremes can be defined quantitatively in two ways:

1) Related to their probability of occurrence

2) Related to a specific (possibly impact-related) threshold.

The first type of definition can either be expressed with respect to given percentiles of the distribution functions of the variables, or with respect to specific return frequencies (e.g., '100-year event'). Compound events can be viewed as a special category of climate extremes, which result from the combination of two or more events, and which are again 'extreme' either from a statistical perspective (tails of distribution functions of climate variables) or associated with a specific threshold (Section 3.1.3.). These two definitions of climate extremes, probability-based or threshold-based, are not necessarily antithetic. Indeed, hazards for society and ecosystems are often extreme both from a probability and 
threshold perspective (e.g., a $40^{\circ} \mathrm{C}$ threshold for midday temperature in the mid-latitudes).

In the scientific literature, several aspects are considered in the definition and analysis of climate extremes (Box 3-1).

\subsubsection{Compound (Multiple) Events}

In climate science, compound events can be (1) two or more extreme events occurring simultaneously or successively, (2) combinations of extreme events with underlying conditions that amplify the impact of the events, or (3) combinations of events that are not themselves extremes but lead to an extreme event or impact when combined. The contributing events can be of similar (clustered multiple events) or different type(s). There are several varieties of clustered multiple events, such as tropical cyclones generated a few days apart with the same path and/or intensities, which may occur if there is a tendency for persistence in atmospheric circulation and genesis conditions. Examples of compound events resulting from events of different types are varied - for instance, high sea level coinciding with tropical cyclone landfall (Section 3.4.4), or cold and dry conditions (e.g., the Mongolian Dzud, see Case Study 9.2.4), or the impact of hot events and droughts on wildfire (Case Study 9.2.2), or a combined risk of flooding from sea level surges and precipitation-induced high river discharge (Svensson and Jones, 2002; Van den Brink et al., 2005). Compound events can even result from 'contrasting extremes', for example, the projected occurrence of both droughts and heavy precipitation events in future climate in some regions (Table 3-3).

Impacts on the physical environment (Section 3.5) are often the result of compound events. For instance, floods will more likely occur over saturated soils (Section 3.5.2), which means that both soil moisture status and precipitation intensity play a role. The wet soil may itself be the result of a number of above-average but not necessarily extreme precipitation events, or of enhanced snow melt associated with temperature anomalies in a given season. Similarly, droughts are the result of pre-existing soil moisture deficits and of the accumulation of precipitation deficits and/or evapotranspiration excesses (Box 3-3), not all (or none) of which are necessarily extreme for a particular drought event when considered in isolation. Also, impacts on human systems or ecosystems (Chapter 4) can be the results of compound events, for example, in the case of health-related impacts associated with combined temperature and humidity conditions (Box 3-1).

Although compound events can involve causally unrelated events, the following causes may lead to a correlation between the occurrence of extremes (or their impacts):

1) A common external forcing factor for changing the probability of the two events (e.g., regional warming, change in frequency or intensity of El Niño events)

2) Mutual reinforcement of one event by the other and vice versa due to system feedbacks (Section 3.1.4)
3) Conditional dependence of the occurrence or impact of one event on the occurrence of another event (e.g., extreme soil moisture levels and precipitation conditions for floods, droughts, see above).

Changes in one or more of these factors would be required for a changing climate to induce changes in the occurrence of compound events. Unfortunately, investigation of possible changes in these factors has received little attention. Also, much of the analysis of changes of extremes has, up to now, focused on individual extremes of a single variable. However, recent literature in climate research is starting to consider compound events and explore appropriate methods for their analysis (e.g., Coles, 2001; Beirlant et al., 2004; Benestad and Haugen, 2007; Renard and Lang, 2007; Schölzel and Friederichs, 2008; Beniston, 2009b; Tebaldi and Sanso, 2009; Durante and Salvadori, 2010).

\subsubsection{Feedbacks}

A special case of compound events is related to the presence of feedbacks within the climate system, that is, mutual interaction between several climate processes, which can either lead to a damping (negative feedback) or enhancement (positive feedback) of the initial response to a given forcing (see also 'climate feedback' in the Glossary). Feedbacks can play an important role in the development of extreme events, and in some cases two (or more) climate extremes can mutually strengthen one another. One example of positive feedback between two extremes is the possible mutual enhancement of droughts and heat waves in transitional regions between dry and wet climates. This feedback has been identified as having an influence on projected changes in temperature variability and heat wave occurrence in Central and Eastern Europe and the Mediterranean (Seneviratne et al., 2006a; Diffenbaugh et al., 2007), and possibly also in Britain, Eastern North America, the Amazon, and East Asia (Brabson et al., 2005; Clark et al., 2006). Further results also suggest that it is a relevant factor for past heat waves and temperature extremes in Europe and the United States (Durre et al., 2000; Fischer et al., 2007a,b; Hirschi et al., 2011). Two main mechanisms that have been suggested to underlie this feedback are: (1) enhanced soil drying during heat waves due to increased evapotranspiration (as a consequence of higher vapor pressure deficit and higher incoming radiation); and (2) higher relative heating of the air from sensible heat flux when soil moisture deficit starts limiting evapotranspiration/latent heat flux (e.g., Seneviratne et al., 2010). Additionally, there may also be indirect and/or non-local effects of dryness on heat waves through, for example, changes in circulation patterns or dry air advection (e.g., Fischer et al., 2007a; Vautard et al., 2007; Haarsma et al., 2009). However, the strength of these feedbacks is still uncertain in current climate models (e.g., Clark et al., 2010), in particular if additional feedbacks with precipitation (e.g., Koster et al., 2004b; Seneviratne et al., 2010) and with land use and land cover state and changes (e.g., Lobell et al., 2008; Pitman et al., 2009; Teuling et al., 2010) are considered. Also, feedbacks between trends in snow cover and changes in temperature extremes have been highlighted as being relevant for projections (e.g., Kharin et al., 2007; Orlowsky and Seneviratne, 2011). Feedbacks with soil moisture 
Table 3-1 | Overview of considered extremes and summary of observed and projected changes at a global scale. Regional details on observed and projected changes in temperature and precipitation extremes are provided in Tables 3-2 and 3-3. Extremes (e.g., cold/warm days/nights, heat waves, heavy precipitation events) are defined with respect to late 20thcentury climate (see also Box 3-1 for discussion of reference period).

\begin{tabular}{|c|c|c|c|c|}
\hline & & Observed Changes (since 1950) & $\begin{array}{l}\text { Attribution of Observed } \\
\text { Changes }\end{array}$ & $\begin{array}{l}\text { Projected Changes (up to 2100) with } \\
\text { Respect to Late 20th Century }\end{array}$ \\
\hline \multirow[t]{3}{*}{$\begin{array}{l}\text { Weather } \\
\text { and } \\
\text { Climate } \\
\text { Variables }\end{array}$} & $\begin{array}{l}\text { Temperature } \\
\text { (Section 3.3.1) }\end{array}$ & $\begin{array}{l}\text { Very likely decrease in number of unusually cold days } \\
\text { and nights at the global scale. Very likely increase in } \\
\text { number of unusually warm days and nights at the } \\
\text { global scale. Medium confidence in increase in length } \\
\text { or number of warm spells or heat waves in many (but } \\
\text { not all) regions. Low or medium confidence in trends in } \\
\text { temperature extremes in some subregions due either } \\
\text { to lack of observations or varying signal within } \\
\text { subregions. [Regional details in Table 3-2] }\end{array}$ & $\begin{array}{l}\text { Likely anthropogenic influence on } \\
\text { trends in warm/cold days/nights at } \\
\text { the global scale. No attribution of } \\
\text { trends at a regional scale with a } \\
\text { few exceptions. }\end{array}$ & $\begin{array}{l}\text { Virtually certain decrease in frequency and magnitude } \\
\text { of unusually cold days and nights at the global scale. } \\
\text { Virtually certain increase in frequency and magnitude } \\
\text { of unusually warm days and nights at the global scale. } \\
\text { Very likely increase in length, frequency, and/or } \\
\text { intensity of warm spells or heat waves over most land } \\
\text { areas. [Regional details in Table 3-3] }\end{array}$ \\
\hline & $\begin{array}{l}\text { Precipitation } \\
\text { (Section 3.3.2) }\end{array}$ & $\begin{array}{l}\text { Likely statistically significant increases in the number } \\
\text { of heavy precipitation events (e.g., 95th percentile) in } \\
\text { more regions than those with statistically significant } \\
\text { decreases, but strong regional and subregional } \\
\text { variations in the trends. [Regional details in Table 3-2] }\end{array}$ & $\begin{array}{l}\text { Medium confidence that } \\
\text { anthropogenic influences have } \\
\text { contributed to intensification of } \\
\text { extreme precipitation at the global } \\
\text { scale. }\end{array}$ & $\begin{array}{l}\text { Likely increase in frequency of heavy precipitation } \\
\text { events or increase in proportion of total rainfall from } \\
\text { heavy falls over many areas of the globe, in particular } \\
\text { in the high latitudes and tropical regions, and in } \\
\text { winter in the northern mid-latitudes. [Regional details } \\
\text { in Table 3-3] }\end{array}$ \\
\hline & $\begin{array}{l}\text { Winds } \\
\text { (Section 3.3.3) }\end{array}$ & Low confidence in trends due to insufficient evidence. & $\begin{array}{l}\text { Low confidence in the causes of } \\
\text { trends due to insufficient evidence. }\end{array}$ & $\begin{array}{l}\text { Low confidence in projections of extreme winds (with } \\
\text { the exception of wind extremes associated with } \\
\text { tropical cyclones). }\end{array}$ \\
\hline \multirow{4}{*}{$\begin{array}{l}\text { Phenomena } \\
\text { Related to } \\
\text { Weather and } \\
\text { Climate } \\
\text { Extremes }\end{array}$} & $\begin{array}{l}\text { Monsoons } \\
\text { (Section 3.4.1) }\end{array}$ & $\begin{array}{l}\text { Low confidence in trends because of insufficient } \\
\text { evidence. }\end{array}$ & $\begin{array}{l}\text { Low confidence due to insufficient } \\
\text { evidence. }\end{array}$ & $\begin{array}{l}\text { Low confidence in projected changes in monsoons, } \\
\text { because of insufficient agreement between climate } \\
\text { models. }\end{array}$ \\
\hline & $\begin{array}{l}\text { El Niño and } \\
\text { other Modes of } \\
\text { Variability } \\
\text { (Sections 3.4.2 } \\
\text { and 3.4.3) }\end{array}$ & $\begin{array}{l}\text { Medium confidence in past trends toward more } \\
\text { frequent central equatorial Pacific El Niño-Southern } \\
\text { Oscillation (ENSO) events. } \\
\text { Insufficient evidence for more specific statements on } \\
\text { ENSO trends. } \\
\text { Likely trends in Southern Annular Mode (SAM). }\end{array}$ & $\begin{array}{l}\text { Likely anthropogenic influence on } \\
\text { identified trends in SAM. }^{1} \\
\text { Anthropogenic influence on trends } \\
\text { in North Atlantic Oscillation (NAO) } \\
\text { are about as likely as not. No } \\
\text { attribution of changes in ENSO. }\end{array}$ & $\begin{array}{l}\text { Low confidence in projections of changes in behavior } \\
\text { of ENSO and other modes of variability because of } \\
\text { insufficient agreement of model projections. }\end{array}$ \\
\hline & $\begin{array}{l}\text { Tropical } \\
\text { Cyclones } \\
\text { (Section 3.4.4) }\end{array}$ & $\begin{array}{l}\text { Low confidence that any observed long-term (i.e., } 40 \\
\text { years or more) increases in tropical cyclone activity are } \\
\text { robust, after accounting for past changes in observing } \\
\text { capabilities. }\end{array}$ & $\begin{array}{l}\text { Low confidence in attribution of } \\
\text { any detectable changes in tropical } \\
\text { cyclone activity to anthropogenic } \\
\text { influences (due to uncertainties in } \\
\text { historical tropical cyclones record, } \\
\text { incomplete understanding of } \\
\text { physical mechanisms, and degree } \\
\text { of tropical cyclone variability). }\end{array}$ & $\begin{array}{l}\text { Likely decrease or no change in frequency of tropical } \\
\text { cyclones. } \\
\text { Likely increase in mean maximum wind speed, but } \\
\text { possibly not in all basins. } \\
\text { Likely increase in heavy rainfall associated with } \\
\text { tropical cyclones. }\end{array}$ \\
\hline & $\begin{array}{l}\text { Extratropical } \\
\text { Cyclones } \\
\text { (Section 3.4.5) }\end{array}$ & $\begin{array}{l}\text { Likely poleward shift in extratropical cyclones. } \\
\text { Low confidence in regional changes in intensity. }\end{array}$ & $\begin{array}{l}\text { Medium confidence in an } \\
\text { anthropogenic influence on } \\
\text { poleward shift. }\end{array}$ & $\begin{array}{l}\text { Likely impacts on regional cyclone activity but low } \\
\text { confidence in detailed regional projections due to only } \\
\text { partial representation of relevant processes in current } \\
\text { models. } \\
\text { Medium confidence in a reduction in the numbers of } \\
\text { mid-latitude storms. } \\
\text { Medium confidence in projected poleward shift of } \\
\text { mid-latitude storm tracks. }\end{array}$ \\
\hline \multirow[t]{2}{*}{$\begin{array}{l}\text { Impacts on } \\
\text { Physical } \\
\text { Environment }\end{array}$} & $\begin{array}{l}\text { Droughts } \\
\text { (Section 3.5.1) }\end{array}$ & $\begin{array}{l}\text { Medium confidence that some regions of the world } \\
\text { have experienced more intense and longer droughts, } \\
\text { in particular in southern Europe and West Africa, but } \\
\text { opposite trends also exist. [Regional details in Table } \\
3-2 \text { ] }\end{array}$ & $\begin{array}{l}\text { Medium confidence that } \\
\text { anthropogenic influence has } \\
\text { contributed to some observed } \\
\text { changes in drought patterns. } \\
\text { Low confidence in attribution of } \\
\text { changes in drought at the level of } \\
\text { single regions due to inconsistent } \\
\text { or insufficient evidence. }\end{array}$ & $\begin{array}{l}\text { Medium confidence in projected increase in duration } \\
\text { and intensity of droughts in some regions of the } \\
\text { world, including southern Europe and the } \\
\text { Mediterranean region, central Europe, central North } \\
\text { America, Central America and Mexico, northeast } \\
\text { Brazil, and southern Africa. } \\
\text { Overall low confidence elsewhere because of } \\
\text { insufficient agreement of projections. } \\
\text { [Regional details in Table 3-3] }\end{array}$ \\
\hline & $\begin{array}{l}\text { Floods } \\
\text { (Section 3.5.2) }\end{array}$ & $\begin{array}{l}\text { Limited to medium evidence available to assess } \\
\text { climate-driven observed changes in the magnitude } \\
\text { and frequency of floods at regional scale. } \\
\text { Furthermore, there is low agreement in this evidence, } \\
\text { and thus overall low confidence at the global scale } \\
\text { regarding even the sign of these changes. } \\
\text { High confidence in trend toward earlier occurrence of } \\
\text { spring peak river flows in snowmelt- and glacier-fed } \\
\text { rivers. }\end{array}$ & $\begin{array}{l}\text { Low confidence that anthropogenic } \\
\text { warming has affected the } \\
\text { magnitude or frequency of floods at } \\
\text { a global scale. } \\
\text { Medium confidence to high } \\
\text { confidence in anthropogenic } \\
\text { influence on changes in some } \\
\text { components of the water cycle } \\
\text { (precipitation, snowmelt) affecting } \\
\text { floods. }\end{array}$ & $\begin{array}{l}\text { Low confidence in global projections of changes in } \\
\text { flood magnitude and frequency because of insufficient } \\
\text { evidence. } \\
\text { Medium confidence (based on physical reasoning) } \\
\text { that projected increases in heavy precipitation would } \\
\text { contribute to rain-generated local flooding in some } \\
\text { catchments or regions. } \\
\text { Very likely earlier spring peak flows in snowmelt- and } \\
\text { glacier-fed rivers. }\end{array}$ \\
\hline
\end{tabular}


Table 3-1 (continued)

\begin{tabular}{|c|c|c|c|c|}
\hline & & Observed Changes (since 1950) & $\begin{array}{l}\text { Attribution of Observed } \\
\text { Changes }\end{array}$ & $\begin{array}{l}\text { Projected Changes (up to } 2100 \text { ) with } \\
\text { Respect to Late 20th Century }\end{array}$ \\
\hline \multirow[t]{2}{*}{$\begin{array}{l}\text { Impacts on } \\
\text { Physical } \\
\text { Environment } \\
\text { (Continued) }\end{array}$} & $\begin{array}{l}\text { Extreme Sea } \\
\text { Level and } \\
\text { Coastal Impacts } \\
\text { (Sections 3.5.3, } \\
\text { 3.5.4, and 3.5.5) }\end{array}$ & $\begin{array}{l}\text { Likely increase in extreme coastal high water } \\
\text { worldwide related to increases in mean sea level in } \\
\text { the late } 20 \text { th century. }\end{array}$ & $\begin{array}{l}\text { Likely anthropogenic influence via } \\
\text { mean sea level contributions. }\end{array}$ & $\begin{array}{l}\text { Very likely that mean sea level rise will contribute to } \\
\text { upward trends in extreme coastal high water levels. } \\
\text { High confidence that locations currently experiencing } \\
\text { coastal erosion and inundation will continue to do so } \\
\text { due to increasing sea level, in the absence of changes } \\
\text { in other contributing factors. }\end{array}$ \\
\hline & $\begin{array}{l}\text { Other Physical } \\
\text { Impacts } \\
\text { (Sections 3.5.6, } \\
\text { 3.5.7, and 3.5.8) }\end{array}$ & $\begin{array}{l}\text { Low confidence in global trends in large landslides in } \\
\text { some regions. Likely increased thawing of permafrost } \\
\text { with likely resultant physical impacts. }\end{array}$ & $\begin{array}{l}\text { Likely anthropogenic influence on } \\
\text { thawing of permafrost. } \\
\text { Low confidence of other } \\
\text { anthropogenic influences because } \\
\text { of insufficient evidence for trends in } \\
\text { other physical impacts in cold } \\
\text { regions. }\end{array}$ & $\begin{array}{l}\text { High confidence that changes in heat waves, glacial } \\
\text { retreat, and/or permafrost degradation will affect high } \\
\text { mountain phenomena such as slope instabilities, mass } \\
\text { movements, and glacial lake outburst floods. High } \\
\text { confidence that changes in heavy precipitation will } \\
\text { affect landslides in some regions. } \\
\text { Low confidence in projected future changes in dust } \\
\text { activity. }\end{array}$ \\
\hline
\end{tabular}

Notes: 1. Due to trends in stratospheric ozone concentrations.

and snow affect extremes in specific regions (hot extremes in transitional climate regions, and cold extremes in snow-covered regions), where they may induce significant deviations in changes in extremes versus changes in the average climate, as also discussed in Section 3.1.6. Other relevant feedbacks involving extreme events are those that can lead to impacts on the global climate, such as modification of land carbon uptake due to enhanced drought occurrence (e.g., Ciais et al., 2005; Friedlingstein et al., 2006; Reichstein et al., 2007) or carbon release due to permafrost degradation (see Section 3.5.7). These aspects are not, however, specifically considered in this chapter (but see Section 3.1.7, on projections of possible increased Amazon drought and forest dieback in this region). Chapter 4 also addresses feedback loops between droughts, fire, and climate change (Section 4.2.2.1).

\subsubsection{Confidence and Likelihood of Assessed Changes in Extremes}

In this chapter, all assessments regarding past or projected changes in extremes are expressed following the new IPCC Fifth Assessment Report uncertainty guidance (Mastrandrea et al., 2010). The new uncertainty guidance makes a clearer distinction between confidence and likelihood (see Box SPM.2). Its use complicates comparisons between assessments in this chapter and those in the IPCC Fourth Assessment Report (AR4), as they are not directly equivalent in terms of nomenclature. The following procedure was adopted in this chapter (see in particular the Executive Summary and Tables 3-1, 3-2, and 3-3.):

- For each assessment, the confidence level for the given assessment is first assessed (low, medium, or high), as discussed in the next paragraph.

- For assessments with high confidence, likelihood assessments of a direction of change are also provided (virtually certain for 99-100\%, very likely for $90-100 \%$, likely for $66-100 \%$, more likely than not for $50-100 \%$, about as likely as not for 33-66\%, unlikely for 0-33\%, very unlikely for $0-10 \%$, and exceptionally unlikely for $0-1 \%$ ). In a few cases for which there is high confidence (e.g., based on physical understanding) but for which there are not sufficient model projections to provide a more detailed likelihood assessment (such as 'likely'), only the confidence assessment is provided.

- For assessments with medium confidence, a direction of change is provided, but without an assessment of likelihood.

- For assessments with low confidence, no direction of change is generally provided.

The confidence assessments are expert-based evaluations that consider the confidence in the tools and data basis (models, data, proxies) used to assess or project changes in a specific element, and the associated level of understanding. Examples of cases of low confidence for model projections are if models display poor performance in simulating the specific extreme in the present climate (see also Box 3-2), or if insufficient literature on model performance is available for the specific extreme, for example, due to lack of observations. Similarly for observed changes, the assessment may be of low confidence if the available evidence is based only on scattered data (or publications) that are insufficient to provide a robust assessment for a large region, or the observations may be of poor quality, not homogeneous, or only of an indirect nature (proxies). In cases with low confidence regarding past or projected changes in some extremes, we indicate whether the low confidence is due to lack of literature, lack of evidence (data, observations), or lack of understanding. It should be noted that there are some overlaps between these categories, as for instance a lack of evidence can be at the root of a lack of literature and understanding. Cases of changes in extremes for which confidence in the models and data is rated as 'medium' are those where we have some confidence in the tools and evidence available to us, but there remain substantial doubts about some aspects of the quality of these tools. It should be noted that an assessment of low confidence in observed or projected changes or trends in a specific extreme neither implies nor excludes the possibility of changes in this extreme. Rather the assessment indicates low confidence in the ability to detect or project any such changes.

Changes (observed or projected) in some extremes are easier to assess than in others either due to the complexity of the underlying processes or to the amount of evidence available for their understanding. This 
results in differing levels of uncertainty in climate simulations and projections for different extremes (Box 3-2). Because of these issues, projections in some extremes are difficult or even impossible to provide, although projections in some other extremes have a high level of confidence. In addition, uncertainty in projections also varies over different time frames for individual extremes, because of varying contributions over time of internal climate variability, model uncertainty, and emission scenario uncertainty to the overall uncertainty (Box 3-2 and Section 3.2). Overall, we can infer that our confidence in past and future changes in extremes varies with the type of extreme, the data available, and the region, season, and time frame being considered, linked with the level of understanding and reliability of simulation of the underlying physical processes. These various aspects are addressed in more detail in Box 3-2, Section 3.2, and the subsections on specific extremes in Sections 3.3-3.5.

\subsubsection{Changes in Extremes and Their Relationship to Changes in Regional and Global Mean Climate}

Changes in extremes can be linked to changes in the mean, variance, or shape of probability distributions, or all of these (see, e.g., Figure 1-2). Thus a change in the frequency of occurrence of hot days (i.e., days above a certain threshold) can arise from a change in the mean daily maximum temperature, and/or from a change in the variance and/or shape of the frequency distribution of daily maximum temperatures. If changes in the frequency of occurrence of hot days were mainly linked to changes in the mean daily maximum temperature, and changes in the shape and variability of the distribution of daily maximum temperatures were of secondary importance, then it might be reasonable to use projected changes in mean temperature to estimate how changes in extreme temperatures might change in the future. If, however, changes in the shape and variability of the frequency distribution of daily maximum temperature were important, such naive extrapolation would be less appropriate or possibly even misleading (e.g., Ballester et al., 2010). The results of both empirical and model studies indicate that although in several situations changes in extremes do scale closely with changes in the mean (e.g., Griffiths et al., 2005), there are sufficient exceptions from this that changes in the variability and shape of probability distributions of weather and climate variables need to be considered as well as changes in means, if we are to project future changes in extremes (e.g., Hegerl et al., 2004; Schär et al., 2004; Caesar et al., 2006; Clark et al., 2006; Della-Marta et al., 2007a; Kharin et al., 2007; Brown et al., 2008; Ballester et al., 2010; Orlowsky and Seneviratne, 2011). This appears to be especially the case for short-duration precipitation, and for temperatures in mid- and high latitudes (but not all locations in these regions). In midand high latitudes stronger increases (or decreases) in some extremes are generally associated with feedbacks with soil moisture or snow cover (Section 3.1.4). Note that the respective importance of changes in mean versus changes in variability also depends on the choice of the reference period used to define the extremes (Box 3-1).

An additional relevant question is the extent to which regional changes in extremes scale with changes in global mean climate. Indeed, recent publications and the public debate have focused, for example, on global mean temperature targets (e.g., Allen et al., 2009; Meinshausen et al., 2009), however, the exact implications of these mean global changes (e.g., ' $2{ }^{\circ} \mathrm{C}$ target') for regional extremes have not been widely assessed (e.g., Clark et al., 2010). Orlowsky and Seneviratne (2011) investigated the scaling between projected changes in the 10th and 90th percentile of Tmax on annual and seasonal (June-July-August: JJA, DecemberJanuary-February: DJF) time scales with globally averaged annual mean changes in Tmax based on the whole CMIP3 ensemble (see Section 3.2.3 for discussion of the CMIP3 ensemble). The results highlight particularly large projected changes in the 10th percentile Tmax in the northern high-latitude regions in winter and the 90th percentile Tmax in Southern Europe in summer with scaling factors of about 2 in both cases (i.e., increases of about $4^{\circ} \mathrm{C}$ for a mean global increase of $2^{\circ} \mathrm{C}$ ). However, in some regions and seasons, the scaling can also be below 1 (e.g., changes in 10th percentile in JJA in the high latitudes). This is also illustrated in Figure 3-5a, which compares analyses of changes in return values of annual extremes of maximum daily temperatures for the overall land and specific regions, and shows high region-to-region variability in these changes. The changes in return values at the global scale ('Globe (Land only)') for their part are almost identical to the changes in global mean daily maximum temperature, suggesting that the scaling issues are related to regional effects rather than overall differences in the changes in the tails versus the means of the distributions of daily maximum temperature. The situation is very different for precipitation (Figure 3-7a), with clearly distinct behavior between changes in mean and extreme precipitation at the global scale, highlighting the dependency of any scaling on the variable being considered. The lack of consistent scaling between regional and seasonal changes in extremes and changes in means has also been highlighted in empirical studies (e.g., Caesar et al., 2006). It should further be noted that not only do regional extremes not necessarily scale with global mean changes, but also mean global warming does not exclude the possibility of cooling in some regions and seasons, both in the recent past and in the coming decades: it has for instance been recently suggested that the decrease in sea ice caused by the mean warming could induce, although not systematically, more frequent cold winter extremes over northern continents (Petoukhov and Semenov, 2010). Also parts of central North America and the eastern United States present cooling trends in mean temperature and some temperature extremes in the spring to summer season in recent decades (Section 3.3.1). It should be noted that, independently of scaling issues for the means and extremes of the same variable, some extremes can be related to mean climate changes in other variables, such as links between mean global changes in relative humidity and some regional changes in heavy precipitation events (Sections 3.2.2.1 and 3.3.2).

Global-scale trends in a specific extreme may be either more reliable or less reliable than some regional-scale trends, depending on the geographical uniformity of the trends in the specific extreme. In particular, climate projections for some variables are not consistent, even in the sign of the projected change, everywhere across the globe (e.g., Christensen et al., 2007; Meehl et al., 2007b). For instance, projections typically include some regions with a tendency toward wetter conditions and others with 
a tendency toward drier conditions, with some regions displaying a shift in climate regimes (e.g., from humid to transitional or transitional to dry). Some of these regional changes will depend on how forcing changes may alter the regional atmospheric circulation, especially in coastal regions and regions with substantial orography. Hence for certain extremes such as floods and droughts, regional projections might indicate larger changes than is the case for projections of global averages (which would average the regional signals exhibiting changes of opposite signs). This also means that signals at the regional scale may be more reliable (and meaningful) in some cases than assessments at the global scale. On the other hand, temperature extremes projections, which are consistent across most regions, are thus more reliable at the global scale ('virtually certain') than at the regional scale (at most 'very likely').

\subsubsection{Surprises / Abrupt Climate Change}

This report focuses on the most probable changes in extremes based on current knowledge. However, the possible future occurrence of lowprobability, high-impact scenarios associated with the crossing of poorly understood climate thresholds cannot be excluded, given the transient and complex nature of the climate system. Such scenarios have important implications for society as highlighted in Section 8.5.1. So, an assessment that we have low confidence in projections of a specific extreme, or even lack of consideration of given climate changes under the categories covered in this chapter (e.g., shutdown of the meridional overturning circulation), should not be interpreted as meaning that no change is expected in this extreme or climate element (see also Section 3.1.5). Feedbacks play an important role in either damping or enhancing extremes in several climate variables (Section 3.1.4), and this can also lead to 'surprises,' that is, changes in extremes greater (or less) than might be expected with a gradual warming of the climate system. Similarly, as discussed in 3.1.3, contrasting or multiple extremes can occur but our understanding of these is insufficient to provide credible comprehensive projections of risks associated with such combinations.

One aspect that we do not address in this chapter is the existence of possible tipping points in the climate system (e.g., Meehl et al., 2007b; Lenton et al., 2008; Scheffer et al., 2009), that is, the risks of abrupt, possibly irreversible changes in the climate system. Abrupt climate change is defined as follows in the Glossary: "The nonlinearity of the climate system may lead to abrupt climate change, sometimes called rapid climate change, abrupt events, or even surprises. The term abrupt often refers to time scales faster than the typical time scale of the responsible forcing. However, not all abrupt climate changes need be externally forced. Some changes may be truly unexpected, resulting from a strong, rapidly changing forcing of a nonlinear system." Thresholds associated with tipping points may be termed 'critical thresholds,' or, in the case of the climate system, 'climate thresholds'. Scheffer et al. (2009) illustrate the possible equilibrium responses of a system to forcing. In the case of a linear response, only a large forcing can lead to a major state change in the system. However, in the presence of a critical threshold even a small change in forcing can lead to a similar major change in the system. For systems with critical bifurcations in the equilibrium state function two alternative stable conditions may exist, whereby an induced change may be irreversible. Such critical transitions within the climate system represent typical low-probability, high-impact scenarios, which were also noted in the AR4 (Meehl et al., 2007b). Lenton et al. (2008) provided a recent review on potential tipping elements within the climate system, that is, subsystems of the Earth system that are at least subcontinental in scale and which may entail a tipping point. Some of these would be especially relevant to certain extremes [e.g., El Niño-Southern Oscillation (ENSO), the Indian summer monsoon, and the Sahara/Sahel and West African monsoon for drought and heavy precipitation, and the Greenland and West Antarctic ice sheets for sea level extremes], or are induced by changes in extremes (e.g., Amazon rainforest die-back induced by drought). For some of the identified tipping elements, the existence of bistability has been suggested by paleoclimate records, but is still debated in some cases (e.g., Brovkin et al., 2009). There is often a lack of agreement between models regarding these low-probability, high-impact scenarios, for instance, regarding a possible increased drought and consequent die-back of the Amazon rainforest (e.g., Friedlingstein et al., 2006; Poulter et al., 2010; see Table 3-3 for dryness projections in this region), the risk of an actual shutdown of the Atlantic thermohaline circulation (e.g., Rahmstorf et al., 2005; Lenton et al., 2008), or the potential irreversibility of the decrease in Arctic sea ice (Tietsche et al., 2011). For this reason, confidence in these scenarios is assessed as low.

\subsection{Requirements and Methods for Analyzing Changes in Extremes}

\subsubsection{Observed Changes}

Sections 3.3 to 3.5 of this chapter provide assessments of the literature regarding changes in extremes in the observed record published mainly since the AR4 and building on the AR4 assessment. Summaries of these assessments are provided in Table 3-1. Overviews of observed regional changes in temperature and precipitation extremes are provided in Table 3-2. In this section issues are discussed related to the data and observations used to examine observed changes in extremes.

Issues with data availability are especially critical when examining changes in extremes of given climate variables (Nicholls, 1995). Indeed, the more rare the event, the more difficult it is to identify long-term changes, simply because there are fewer cases to evaluate (Frei and Schär, 2001; Klein Tank and Können, 2003). Identification of changes in extremes is also dependent on the analysis technique employed ( $X$. Zhang et al., 2004; Trömel and Schönwiese, 2005). Another important criterion constraining data availability for the analysis of extremes is the respective time scale on which they occur (Section 3.1.2), since this determines the required temporal resolution for their assessment (e.g., heavy hourly or daily precipitation versus multi-year drought). Longer time resolution data (e.g., monthly, seasonal, and annual values) for temperature and precipitation are available for most parts of the world 
starting late in the 19th to early 20th century, and allow analysis of meteorological drought (see Box 3-3) and unusually wet periods of the order of a month or longer. To examine changes in extremes occurring on short time scales, particularly of climate variables such as temperature and precipitation (or wind), normally requires the use of high-temporal resolution data, such as daily or sub-daily observations, which are generally either not available, or available only since the middle of the 20th century and in many regions only from as recently as 1970 . Even where sufficient data are available, several problems can still limit their analysis. First, although the situation is changing (especially for the situation with respect to 'extreme indices,' Box 3-1), many countries still do not freely distribute their higher temporal resolution data. Second, there can be issues with the quality of measurements. $A$ third important issue is climate data homogeneity (see below). These and other issues are discussed in detail in the AR4 (Trenberth et al., 2007). For instance, the temperature and precipitation stations considered in the daily data set used in Alexander et al. (2006) are not globally uniform. Although observations for most parts of the globe are available, measurements are lacking in Northern South America, Africa, and part of Australia. The other data set commonly used for extremes analyses is from Caesar et al. (2006; used, e.g., in Brown et al., 2008), which also has data gaps in most of South America, Africa, Eastern Europe, Mexico, the Middle East, India, and Southeast Asia. Also the study by Vose et al. (2005) has data gaps in South America, Africa, and India. It should be further noted that the regions with data coverage do not all have the same density of stations (Alexander et al., 2006; Caesar et al., 2006). While some studies are available on a country or regional basis for areas not covered in global studies (see, e.g., Tables 3-2 and 3-3), lack of data in many parts of the globe leads to limitations in our ability to assess observed changes in climate extremes for many regions.

Whether or not climate data are homogeneous is of clear relevance for an analysis of extremes, especially at smaller spatial scales. Data are defined as homogeneous when the variations and trends in a climate time series are due solely to variability and changes in the climate system. Some meteorological elements are especially vulnerable to uncertainties caused by even small changes in the exposure of the measuring equipment. For instance, erection of a small building or changes in vegetative cover near

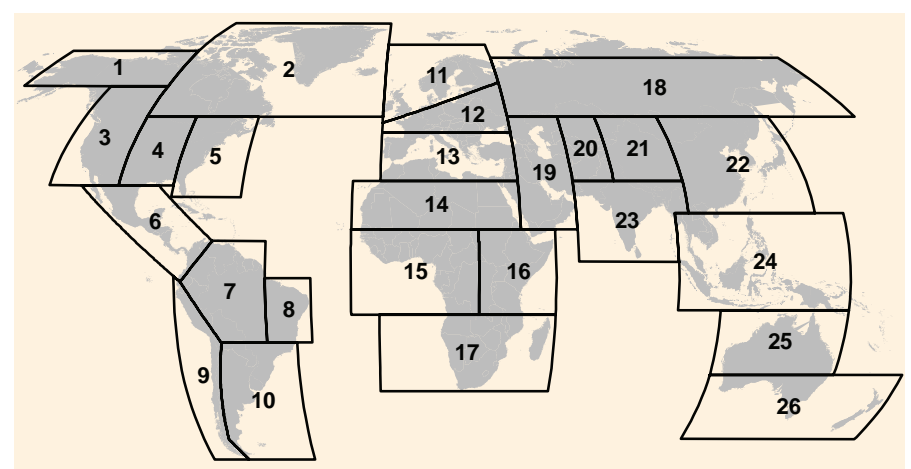

Figure 3-1 | Definitions of regions used in Tables 3-2 and 3-3, and Figures 3-5 and 3-7. Exact coordinates of the regions are provided in the on-line supplement, Appendix 3.A. Assessments and analyses are provided for land areas only. the measuring equipment can produce a bias in wind measurements (Wan et al., 2010). When a change occurs it can result in either a discontinuity in the time series (step change) or a more gradual change that can manifest itself as a false trend (Menne and Williams Jr., 2009), both of which can impact on whether a particular observation exceeds a threshold. Homogeneity detection and data adjustments have been implemented for longer averaging periods (e.g., monthly, seasonal, annual); however, techniques applicable to shorter observing periods (e.g., daily) data have only recently been developed (e.g., Vincent et al., 2002; Della-Marta and Wanner, 2006), and have not been widely implemented. Homogeneity issues also affect the monitoring of other meteorological and climate variables, for which further and more severe limitations also can exist. This is in particular the case regarding measurements of wind and relative humidity, and data required for the analysis of weather and climate phenomena (tornadoes, extratropical and tropical cyclones; Sections 3.3.3, 3.4.4, and 3.4.5), as well as impacts on the physical environment (e.g., droughts, floods, cryosphere impacts; Section 3.5).

Thunderstorms, tornadoes, and related phenomena are not well observed in many parts of the world. Tornado occurrence since 1950 in the United States, for instance, displays an increasing trend that mainly reflects increased population density and increased numbers of people in remote areas (Trenberth et al., 2007; Kunkel et al., 2008). Such trends increase the likelihood that a tornado would be observed. A similar problem occurs with thunderstorms. Changes in reporting practices, increased population density, and even changes in the ambient noise level at an observing station all have led to inconsistencies in the observed record of thunderstorms.

Studies examining changes in extratropical cyclones, which focus on changes in storm track location, intensities, and frequency, are limited in time due to a lack of suitable data prior to about 1950. Most of these studies have relied on model-based reanalyses that also incorporate observations into a hybrid model-observational data set. However, reanalyses can have homogeneity problems due to changes in the amount and type of data being assimilated, such as the introduction of satellite data in the late 1970s and other observing system changes (Trenberth et al., 2001; Bengtsson et al., 2004). Recent reanalysis efforts have attempted to produce more homogeneous reanalyses that show promise for examining changes in extratropical cyclones and other climate features (Compo et al., 2006). Results, however, are strongly dependent on the reanalysis and cyclone tracking techniques used (Ulbrich et al., 2009).

The robustness of analyses of observed changes in tropical cyclones has been hampered by a number of issues with the historical record. One of the major issues is the heterogeneity introduced by changing technology and reporting protocols within the responsible agencies (e.g., Landsea et al., 2004). Further heterogeneity is introduced when records from multiple ocean basins are combined to explore global trends, because data quality and reporting protocols vary substantially between agencies (Knapp and Kruk, 2010). Much like other weather and climate observations, 


\section{FAQ 3.1 | Is the Climate Becoming More Extreme?}

While there is evidence that increases in greenhouse gases have likely caused changes in some types of extremes, there is no simple answer to the question of whether the climate, in general, has become more or less extreme. Both the terms 'more extreme' and 'less extreme' can be defined in different ways, resulting in different characterizations of observed changes in extremes. Additionally, from a physical climate science perspective it is difficult to devise a comprehensive metric that encompasses all aspects of extreme behavior in the climate.

One approach for evaluating whether the climate is becoming more extreme would be to determine whether there have been changes in the typical range of variation of specific climate variables. For example, if there was evidence that temperature variations in a given region had become significantly larger than in the past, then it would be reasonable to conclude that temperatures in that region had become more extreme. More simply, temperature variations might be considered to be becoming more extreme if the difference between the highest and the lowest temperature observed in a year is increasing. According to this approach, daily temperature over the globe may have become less extreme because there have generally been greater increases in mean daily minimum temperatures globally than in mean daily maximum temperatures, over the second half of the 20th century. On the other hand, one might conclude that daily precipitation has become more extreme because observations suggest that the magnitude of the heaviest precipitation events has increased in many parts of the world. Another approach would be to ask whether there have been significant changes in the frequency with which climate variables cross fixed thresholds that have been associated with human or other impacts. For example, an increase in the mean temperature usually results in an increase in hot extremes and a decrease in cold extremes. Such a shift in the temperature distribution would not increase the 'extremeness' of day-to-day variations in temperature, but would be perceived as resulting in a more extreme warm temperature climate, and a less extreme cold temperature climate. So the answer to the question posed here would depend on the variable of interest, and on which specific measure of the extremeness of that variable is examined. As well, to provide a complete answer to the above question, one would also have to collate not just trends in single variables, but also indicators of change in complex extreme events resulting from a sequence of individual events, or the simultaneous occurrence of different types of extremes. So it would be difficult to comprehensively describe the full suite of phenomena of concern, or to find a way to synthesize all such indicators into a single extremeness metric that could be used to comprehensively assess whether the climate as a whole has become more extreme from a physical perspective. And to make such a metric useful to more than a specific location, one would have to combine the results at many locations, each with a different perspective on what is 'extreme.'

Continued next page $\longrightarrow$

tropical cyclone observations are taken to support short-term forecasting needs. Improvements in observing techniques are often implemented without any overlap or calibration against existing methods to document the impact of the changes on the climate record. Additionally, advances in technology have enabled better and more complete observations. For example, the introduction of aircraft reconnaissance in some basins in the 1940s and satellite data in the 1960s had a profound effect on our ability to accurately identify and measure tropical cyclones, particularly those that never encountered land or a ship. While aircraft reconnaissance programs have continued in the North Atlantic, they were terminated in the Western Pacific in 1987. The introduction of geostationary satellite imagery in the 1970s, and the introduction (and subsequent improvement) of new tropical cyclone analysis methods (such as the Dvorak technique for estimating storm intensity), further compromises the homogeneity of historical records of tropical cyclone activity.

Regarding impacts to the physical environment, soil moisture is a key variable for which data sets are extremely scarce (e.g., Robock et al., 2000; Seneviratne et al., 2010). This represents a critical issue for the validation and correct representation of soil moisture (agricultural) as well as hydrological drought (Box 3-3) in climate, land surface, and hydrological models, and the monitoring of ongoing changes in regional terrestrial water storage. As a consequence, these need to be inferred from simple climate indices or model-based approaches (Box 3-3). Such estimates rely in large part on precipitation observations, which have, however, inadequate spatial coverage for these applications in many regions of the world (e.g., Oki et al., 1999; Fekete et al., 2004; Koster et al., 2004a). Similarly, runoff observations are not globally available, which results in significant uncertainties in the closing of the global and some regional water budgets (Legates et al., 2005; Peel and McMahon, 2006; Dai et al., 2009; Teuling et al., 2009), as well as for the global analysis of changes in the occurrence of floods (Section 3.5.2). Additionally, ground observations of snow, which are lacking in several regions, are important for the investigation of physical impacts, particularly those related to the cryosphere and runoff generation (e.g., Essery et al., 2009; Rott et al., 2010).

All of the above-mentioned issues lead to uncertainties in observed trends in extremes. In many instances, great care has been taken to develop procedures to reduce the confounding influences of these issues on the data, which in turn helps to reduce uncertainty, and progress has been made in the last 15 years (e.g., Caesar et al., 2006; 
Three types of metrics have been considered to avoid these problems, and thereby allow an answer to this question. One approach is to count the number of record-breaking events in a variable and to examine such a count for any trend. However, one would still face the problem of what to do if, for instance, hot extremes are setting new records, while cold extremes are not occurring as frequently as in the past. In such a case, counting the number of records might not indicate whether the climate was becoming more or less extreme, rather just whether there was a shift in the mean climate. Also, the question of how to combine the numbers of record-breaking events in various extremes (e.g., daily precipitation and hot temperatures) would need to be considered. Another approach is to combine indicators of a selection of important extremes into a single index, such as the Climate Extremes Index (CEI), which measures the fraction of the area of a region or country experiencing extremes in monthly mean surface temperature, daily precipitation, and drought. The CEI, however, omits many important extremes such as tropical cyclones and tornadoes, and could, therefore, not be considered a complete index of 'extremeness.' Nor does it take into account complex or multiple extremes, nor the varying thresholds that relate extremes to impacts in various sectors.

A third approach to solving this dilemma arises from the fact that extremes often have deleterious economic consequences. It may therefore be possible to measure the integrated economic effects of the occurrence of different types of extremes into a common instrument such as insurance payout to determine if there has been an increase or decrease in that instrument. This approach would have the value that it clearly takes into account those extremes with economic consequences. But trends in such an instrument will be dominated by changes in vulnerability and exposure and it will be difficult, if not impossible, to disentangle changes in the instrument caused by non-climatic changes in vulnerability or exposure in order to leave a residual that reflects only changes in climate extremes. For example, coastal development can increase the exposure of populations to hurricanes; therefore, an increase in damage in coastal regions caused by hurricane landfalls will largely reflect changes in exposure and may not be indicative of increased hurricane activity. Moreover, it may not always be possible to associate impacts such as the loss of human life or damage to an ecosystem due to climate extremes to a measurable instrument.

None of the above instruments has yet been developed sufficiently as to allow us to confidently answer the question posed here. Thus we are restricted to questions about whether specific extremes are becoming more or less common, and our confidence in the answers to such questions, including the direction and magnitude of changes in specific extremes, depends on the type of extreme, as well as on the region and season, linked with the level of understanding of the underlying processes and the reliability of their simulation in models.

Brown et al., 2008). As a consequence, more complete and homogenous information about changes is now available for at least some variables and regions (Nicholls and Alexander, 2007; Peterson and Manton, 2008). For instance, the development of global databases of daily temperature and precipitation covering up to $70 \%$ of the global land area has allowed robust analyses of extremes (see Alexander et al., 2006). In addition, analyses of temperature and precipitation extremes using higher temporal resolution data, such as that available in the Global Historical Climatology Network - Daily data set (Durre et al., 2008) have also proven robust at both a global (Alexander et al., 2006) and regional scale (Sections 3.3.1 and 3.3.2). Nonetheless, as highlighted above, for many extremes, data remain sparse and problematic, resulting in lower ability to establish changes, particularly on a global basis and for specific regions.

\subsubsection{The Causes behind the Changes}

This section discusses the main requirements, approaches, and considerations for the attribution of causes for observed changes in extremes. In Sections 3.3 to 3.5, the causes of observed changes in specific extremes are assessed. A global summary of these assessments is provided in Table 3-1. Climate variations and change are induced by variability internal to the climate system, and changes in external forcings, which include natural external forcings such as changes in solar irradiance and volcanism, and anthropogenic forcings such as aerosol and greenhouse gas emissions principally due to the burning of fossil fuels, and land use and land cover changes. The mean state, extremes, and variability are all related aspects of the climate, so external forcings that affect the mean climate would in general result in changes in extremes. For this reason, we provide in Section 3.2.2.1 a brief overview of human-induced changes in the mean climate to aid the understanding of changes in extremes as the literature directly addressing the causes of changes in extremes is quite limited.

\subsubsection{Human-Induced Changes in the Mean Climate that Affect Extremes}

The occurrence of extremes is usually the result of multiple factors, which can act either on the large scale or on the regional (and local) scale (see also Section 3.1.6). Some relevant large-scale impacts of 
external forcings affecting extremes include net increases in temperature induced by changes in radiation, enhanced moisture content of the atmosphere, and increased land-sea contrast in temperatures, which can, for example, affect circulation patterns and to some extent monsoons. At regional and local scales, additional processes can modulate the overall changes in extremes, including regional feedbacks, in particular linked to land-atmosphere interactions with, for example, soil moisture or snow (e.g., Section 3.1.4). This section briefly reviews the current understanding of the causes (i.e., in the sense of attribution to either external forcing or internal climate variability) of large-scale (and some regional) changes in the mean climate that are of relevance to extreme events, to the extent that they have been considered in detection and attribution studies.

Regarding observed increases in global average annual mean surface temperatures in the second half of the 20th century, we base our analysis on the following AR4 assessment (Hegerl et al., 2007): Most of the observed increase in global average temperatures is very likely due to the observed increase in anthropogenic greenhouse gas concentrations. Greenhouse gas forcing alone would likely have resulted in a greater warming than observed if there had not been an offsetting cooling effect from aerosol and other forcings. It is extremely unlikely $(<5 \%)$ that the global pattern of warming can be explained without external forcing, and very unlikely that it is due to known natural external causes alone. Anthropogenically forced warming over the second half of the 20th century has also been detected in ocean heat content and air temperatures in all continents (Hegerl et al., 2007; Gillett et al., 2008b).

Hegerl et al. (2007) assessed literature that considered detection in temperature trends at scales as small as approximately $500 \mathrm{~km}$. Recent work has provided more evidence of detection of an anthropogenic influence at increasingly smaller spatial scales and for seasonal averages (Stott et al., 2010). For instance, Min and Hense (2007) found that estimates of response to anthropogenic forcing from the multi-model Coupled Model Intercomparison Project 3 (CMIP3) ensemble (see Section 3.2.3.3) provided a better explanation for observed continentalscale seasonal temperature changes than alternative explanations such as natural external forcing or internal variability. In another study, an anthropogenic signal was detected in 20th-century summer temperatures in Northern Hemisphere subcontinental regions except central North America, although the results were more uncertain when anthropogenic and natural signals were considered together (Jones et al., 2008). An anthropogenic signal has also been detected in multi-decadal trends in a US climate extreme index (Burkholder and Karoly, 2007), in the hydrological cycle of the western United States (Barnett et al., 2008), in New Zealand temperatures (Dean and Stott, 2009), and in European temperatures (Christidis et al., 2011a).

Attribution has more stringent demands than those for the detection of an external influence in observations. Overall, attribution at scales smaller than continental has still not yet been established primarily due to the low signal-to-noise ratio and the difficulties of separately attributing effects of the wider range of possible driving processes (either attributable to external forcing or internal climate variability) at these scales (Hegerl et al., 2007). One reason is that averaging over smaller regions reduces the internal variability less than does averaging over large regions. In addition, the small-scale details of external forcing, and the responses simulated by models, are less credible than largescale features. For instance, temperature changes are poorly simulated by models in some regions and seasons (Dean and Stott, 2009; van Oldenborgh et al., 2009). Also the inclusion of additional forcing factors, such as land use change and aerosols that can be more important at regional scales, remains a challenge (Lohmann and Feichter, 2007; Pitman et al., 2009; Rotstayn et al., 2009).

One of the significant advances since AR4 is emerging evidence of human influence on global atmospheric moisture content and precipitation. According to the Clausius-Clapeyron relationship, the saturation vapor pressure increases approximately exponentially with temperature. It is physically plausible that relative humidity would remain roughly constant under climate change (e.g., Hegerl et al., 2007). This means that specific humidity increases about $7 \%$ for a one degree increase in temperature in the current climate. Indeed, observations indicate significant increases between 1973 and 2003 in global surface specific humidity but not in relative humidity (Willett et al., 2008), and at the largest spatial-temporal scales moistening is close to the Clausius-Clapeyron scaling of the saturated specific humidity ( 7\% K-1; Willett et al., 2010), though relative humidity over low- and mid-latitude land areas decreased over a 10-year period prior to 2008 possibly due to a slower temperature increase in the oceans than over the land (Simmons et al., 2010). By comparing observations with model simulations, changes in the global surface specific humidity for 1973-2003 (Willett et al., 2007), and in lower tropospheric moisture content over the 1988-2006 period (Santer et al., 2007) can be attributed to anthropogenic influence.

The increase in the atmospheric moisture content would be expected to lead to an increase in extreme precipitation when other factors do not change. Min et al. (2011) detected an anthropogenic influence in annual maxima of daily precipitation over Northern Hemisphere land areas. The influence of anthropogenic forcing has been detected in the latitudinal pattern of land precipitation trends though the model-simulated magnitude of changes is smaller than that observed (X. Zhang et al., 2007). The smaller changes in model simulations may be due in part to averaging precipitation trends from different model simulations, as spatial patterns of trends simulated by different models are not exactly the same. The influence of anthropogenic greenhouse gases and aerosols on changes in precipitation over high-latitude land areas north of $55^{\circ} \mathrm{N}$ has also been detected (Min et al., 2008). Detection is possible there, despite limited data coverage, in part because the response to forcing is relatively strong, and because internal variability in precipitation is low in this region.

\subsubsection{How to Attribute a Change in Extremes to Causes}

The good practice guidance paper on detection and attribution (Hegerl et al., 2010) reconciles terminologies of detection and attribution used 


\title{
FAQ 3.2 | Has Climate Change Affected Individual Extreme Events?
}

\begin{abstract}
A changing climate can be expected to lead to changes in climate and weather extremes. But it is challenging to associate a single extreme event with a specific cause such as increasing greenhouse gases because a wide range of extreme events could occur even in an unchanging climate, and because extreme events are usually caused by a combination of factors. Despite this, it may be possible to make an attribution statement about a specific weather event by attributing the changed probability of its occurrence to a particular cause. For example, it has been estimated that human influences have more than doubled the probability of a very hot European summer like that of 2003.
\end{abstract}

Recent years have seen many extreme events including the extremely hot summer in parts of Europe in 2003 and 2010, and the intense North Atlantic hurricane seasons of 2004 and 2005. Can the increased atmospheric concentrations of greenhouse gases be considered the 'cause' of such extreme events? That is, could we say these events would not have occurred if $\mathrm{CO}_{2}$ had remained at pre-industrial concentrations? For instance, the monthly mean November temperature averaged across the state of New South Wales in Australia for November 2009 is about 3.5 standard deviations warmer than the 1950-2008 mean, suggesting that the chance of such a temperature occurring in the 1950-2008 climate (assuming a stationary climate) is quite low. Is this event, therefore, an indication of a changing climate? In the CRUTEM3V global land surface temperature data set, about one in every 900 monthly mean temperatures observed between 1900 and 1949 lies more than 3.5 standard deviations above the corresponding monthly mean temperature for 1950-2008. ${ }^{1}$ Since global temperature was lower in the first half of the 20th century, this clearly indicates that an extreme warm event as rare as the November 2009 temperature in any specific location could have occurred in the past, even if its occurrence in recent times is more probable.

A second complicating issue is that extreme events usually result from a combination of factors, and this will make it difficult to attribute an extreme to a single causal factor. The hot 2003 European summer was associated with a persistent high-pressure system (which led to clear skies and thus more solar energy received at the surface) and too-dry soil (which meant that less solar energy was used for evaporation, leaving more energy to heat the soil). Another example is that hurricane genesis requires weak vertical wind shear, as well as very warm sea surface temperatures. Since some factors, but not others, may be affected by a specific cause such as increasing greenhouse gas concentrations, it is difficult to separate the human influence on a single, specific extreme event from other factors influencing the extreme.

Nevertheless, climate models can sometimes be used to identify if specific factors are changing the likelihood of the occurrence of extreme events. In the case of the 2003 European heat wave, a model experiment indicated that human influences more than doubled the likelihood of having a summer in Europe as hot as that of 2003, as discussed in the AR4. The value of such a probability-based approach - "Does human influence change the likelihood of an event?" - is that it can be used to estimate the influence of external factors, such as increases in greenhouse gases, on the frequency of specific types of events, such as heat waves or cold extremes. The same likelihood-based approach has been used to examine anthropogenic greenhouse gas contribution to flood probability.

The discussion above relates to an individual, specific occurrence of an extreme event (e.g., a single heat wave). For the reasons outlined above it remains very difficult to attribute any individual event to greenhouse gas-induced warming (even if physical reasoning or model experiments suggest such an extreme may be more likely in a changed climate). On the other hand, a long-term trend in an extreme (e.g., heat wave occurrences) is a different matter. It is certainly feasible to test whether such a trend is likely to have resulted from anthropogenic influences on the climate, just as a global warming trend can be assessed to determine its likely cause.

\footnotetext{
1 We used the CRUTEM3V land surface temperature data. We limit our calculation to grid points with long-term observations, requiring at least 50 non-missing values during 1950-2008 for a calendar month and a grid point to be included. A standard deviation is computed for the period 1950-2008. We then count the number of occurrences when the temperature anomaly during 1900-1949 relative to 1950-2008 mean is greater than 3.5 standard deviations, and compare it with the total number of observations for the grid and month in that period. The ratio of these two numbers is 0.00107 .
}

by Working Groups I and II in the AR4. It provides detailed guidance on the procedures that include two main approaches to attribute a change in climate to causes. One is single-step attribution, which involves assessments that attribute an observed change within a system to an external forcing based on explicitly modelling the response of the variable to the external forcings. The alternate procedure is multi-step attribution, which combines an assessment that attributes an observed change in a variable of interest to a change in climate, with a separate assessment that attributes the change to external forcings. Attribution of changes in climate extremes has some unique issues. Observed data 
are limited in both quantity and quality (Section 3.2.1), resulting in uncertainty in the estimation of past changes; the signal-to-noise ratio may be low for many variables and insufficient data may be available to detect such weak signals. In addition, global climate models (GCMs) have several issues in simulating extremes and downscaling techniques can only partly circumvent these issues (Section 3.2.3).

Single-step attribution based on optimal detection and attribution (e.g., Hegerl et al., 2007) can in principle be applied to climate extremes. However, the difference in statistical properties between mean values and extremes needs to be carefully addressed (e.g., Zwiers et al., 2011; see also Section 3.1.6). Post-processing of climate model simulations to derive a quantity of interest that is not explicitly simulated by the models, by applying empirical methods or physically based models to the outputs from the climate models, may make it possible to directly compare observed extremes with climate model results. For example, sea level pressure simulated by multiple GCMs has been used to derive geostrophic wind to represent atmospheric storminess and to derive significant wave height on the oceans for the detection of external influence on trends in atmospheric storminess and northern oceans wave heights (X.L. Wang et al., 2009a). GCM-simulated precipitation and temperature have also been downscaled as input to hydrological and snowpack models to infer past and future changes in temperature, timing of the peak flow, and snow water equivalent for the western United States, and this enabled a detection and attribution analysis of human-induced changes in these variables (Barnett et al., 2008).

If a single-step attribution of causes to effects on extremes or physical impacts of extremes is not feasible, it might be feasible to conduct a multiple-step attribution. The assessment would then need to be based on evidence not directly derived from model simulations, that is, physical understanding and expert judgment, or their combination. For instance, in the northern high-latitude regions, spring temperature has increased, and the timing of spring peak flows in snowmelt-fed rivers has shifted toward earlier dates (Regonda et al., 2005; Knowles et al., 2006). A change in streamflow may be attributable to external influence if streamflow regime change can be attributed to a spring temperature increase and if the spring temperature increase can be attributed to external forcings (though these changes may not necessarily be linked to changes in floods; Section 3.5.2). If the chain of processes is established (e.g., in this case additionally supported by the physical understanding that snow melts earlier as spring temperature increases), the confidence in the overall assessment would be similar to, or weaker than, the lower confidence in the two steps in the assessment. In cases where the underlying physical mechanisms are less certain, such as those linking tropical cyclones and sea surface temperature (see Section 3.4.4), the confidence in multi-step attribution can be severely undermined. A necessary condition for multi-step attribution is to establish the chain of mechanisms responsible for the specific extremes being considered. Physically based process studies and sensitivity experiments that help the physical understanding (e.g., Findell and Delworth, 2005; Seneviratne et al., 2006a; Haarsma et al., 2009) can possibly play a role in developing such multi-step attributions.
Extreme events are rare, which means that there are also few data available to make assessments regarding changes in their frequency or intensity (Section 3.2.1). When a rare and high-impact meteorological extreme event occurs, a question that is often posed is whether such an event is due to anthropogenic influence. Because it is very difficult to rule out the occurrence of low-probability events in an unchanged climate and because the occurrence of such events usually involves multiple factors, it is very difficult to attribute an individual event to external forcing (Allen, 2003; Hegerl et al., 2007; Dole et al., 2011; see also FAQ 3.2). However, in this case, it may be possible to estimate the influence of external forcing on the likelihood of such an event occurring (e.g., Stott et al., 2004; Pall et al., 2011; Zwiers et al., 2011).

\subsubsection{Projected Long-Term Changes and Uncertainties}

In this section we discuss the requirements and methods used for preparing climate change projections, with a focus on projections of extremes and the associated uncertainties. The discussion draws on the AR4 (Christensen et al., 2007; Meehl et al., 2007b; Randall et al., 2007) with consideration of some additional issues relevant to projections of extremes in the context of risk and disaster management. More detailed assessments of projections for specific extremes are provided in Sections 3.3 to 3.5. Summaries of these assessments are provided in Table 3-1. Overviews of projected regional changes in temperature extremes, heavy precipitation, and dryness are provided in Table 3-3 (see pages 196-202).

\subsubsection{Information Sources for Climate Change Projections}

Work on the construction, assessment, and communication of climate change projections, including regional projections and of extremes, draws on information from four sources: (1) GCMs; (2) downscaling of GCM simulations; (3) physical understanding of the processes governing regional responses; and (4) recent historical climate change (Christensen et al., 2007; Knutti et al., 2010b). At the time of the AR4, GCMs were the main source of globally available regional information on the range of possible future climates including extremes (Christensen et al., 2007). This is still the case for many regions, as can be seen in Table 3-3.

The AR4 concluded that statistics of extreme events for present-day climate, especially temperature, are generally well simulated by current GCMs at the global scale (Randall et al., 2007). Precipitation extremes are, however, less well simulated (Randall et al., 2007; Box 3-2). As they continue to develop, and their spatial resolution as well as their complexity continues to improve, GCMs could become increasingly useful for investigating smaller-scale features, including changes in extreme weather events. However, when we wish to project climate and weather extremes, not all atmospheric phenomena potentially of relevance can be realistically or explicitly simulated. GCMs include a number of approximations, known as parameterizations, of processes (e.g., relating to clouds) that cannot be fully resolved in climate models. Furthermore, 
the assessment of climate model performance with respect to extremes (summarized in Sections 3.3 to 3.5 for specific extremes), particularly at the regional scale, is still limited by the rarity of extreme events that makes evaluation of model performance less robust than is the case for average climate. Evaluation is further hampered by incomplete data on the historical frequency and severity of extremes, particularly for variables other than temperature and precipitation, and for specific regions (Section 3.2.1; Table 3-2).

The requirement for projections of extreme events has provided one of the motivations for the development of regionalization or downscaling techniques (Carter et al., 2007). These have been specifically developed for the study of regional- and local-scale climate change, to simulate weather and climate at finer spatial resolutions than is possible with GCMs - a step that is particularly relevant for many extremes given their spatial scale. These techniques are, nonetheless, constrained by the reliability of large-scale information coming from GCMs. Recent advances in downscaling for extremes are discussed below.

As indicated in the Glossary, downscaling "is a method that derives localto regional-scale (up to $100 \mathrm{~km}$ ) information from larger-scale models or data analyses." Two main methods are distinguished: dynamical downscaling and empirical/statistical downscaling (Christensen et al., 2007). The dynamical method uses the output of regional climate models (RCMs), global models with variable spatial resolution, or highresolution global models. The empirical/statistical methods develop statistical relationships that link the large-scale atmospheric variables with local/regional climate variables. In all cases, the quality of the downscaled product depends on the quality of the driving model. Dynamical and statistical downscaling techniques are briefly introduced hereafter. Specific limitations that need to be considered in the evaluation of projections are also discussed in Section 3.2.3.2.

The most common approach to dynamical downscaling uses highresolution RCMs, currently at scales of 20 to $50 \mathrm{~km}$, but in some cases down to 10 to $15 \mathrm{~km}$ (e.g., Dankers et al., 2007), to represent regional sub-domains, using either observed (reanalysis) or lower-resolution GCM data to provide their boundary conditions. Using non-hydrostatic mesoscale models, applications at 1 - to $5-\mathrm{km}$ resolution are also possible for shorter periods (typically a few months, a few full years at most) - a scale at which clouds and convection can be explicitly resolved and the diurnal cycle tends to be better resolved (e.g., Grell et al., 2000; Hay et al., 2006; Hohenegger et al., 2008; Kanada et al., 2010b). Less commonly used approaches to dynamical downscaling involve the use of stretched-grid (variable resolution) models and high-resolution 'timeslice' models (e.g., Cubasch et al., 1995; Gibelin and Deque, 2003; Coppola and Giorgi, 2005) with the latter including some simulations at 20 km globally (Kamiguchi et al., 2006; Kitoh et al., 2009; Kim et al., 2010). The main advantage of dynamical downscaling is its potential for capturing mesoscale nonlinear effects and providing information for many climate variables at a relatively high spatial resolution, although still not as high as some require. Dynamical downscaling cannot provide information at the point (i.e., weather station) scale (a scale at which the RCM and GCM parameterizations would not work). Like GCMs, RCMs provide precipitation averaged over a grid cell, which means a tendency to more days of light precipitation (Frei et al., 2003; Barring et al., 2006) and reduced magnitude of extremes (Chen and Knutson, 2008; Haylock et al., 2008) compared with point values. These scaling issues need to be considered when evaluating the ability of RCMs and GCMs to simulate precipitation and other extremes.

Statistical downscaling methods use relationships between large-scale fields (predictors) and local-scale surface variables (predictands) that have been derived from observed data, and apply these to equivalent large-scale fields simulated by climate models (Christensen et al., 2007). They may also include weather generators that provide the basis for a number of recently developed user tools that can be used to assess changes in extreme events (Kilsby et al., 2007; Burton et al., 2008; Qian et al., 2008; Semenov, 2008). Statistical downscaling has been demonstrated to have potential in a number of different regions including Europe (e.g., Schmidli et al., 2007), Africa (e.g., Hewitson and Crane, 2006), Australia (e.g., Timbal et al., 2008, 2009), South America (e.g., D'Onofrio et al., 2010) and North America (e.g., Vrac et al., 2007; Dibike et al., 2008). Statistical downscaling methods are able to access finer spatial scales than dynamical methods and can be applied to parameters that cannot be directly obtained from RCMs. Seasonal indices of extremes can, for example, be simulated directly without having to first produce daily time series (Haylock et al., 2006a), or distribution functions of extremes can be simulated (Benestad, 2007). However, statistical downscaling methods require observational data at the desired scale (e.g., the point or station scale) for a long enough period to allow the model to be well trained and validated, and in some methods can lack coherency among multiple climate variables and/or multiple sites. One specific disadvantage of some, but not all, methods based on the analog approach is that they cannot produce extreme events greater in magnitude than have been observed before (Timbal et al., 2009). Moreover, statistical downscaling does not allow for the possibility of future process-based changes in relationships between predictors and predictands (see Section 3.2.3.2). There have been few systematic intercomparisons of dynamical and statistical downscaling approaches focusing on extremes (Fowler et al., 2007b). Two examples focus on extreme precipitation for the United Kingdom (Haylock et al., 2006a) and the Alps (Schmidli et al., 2007), respectively. A few hybrid statisticodynamical downscaling methods also exist, including a two-step approach used to downscale heavy precipitation events in southern France (Beaulant et al., 2011). A conceptually similar cascading technique has also been used to downscale tropical cyclones (Bender et al., 2010; see Section 3.4.4).

In terms of temporal resolution, while GCMs and RCMs operate at sub-daily time steps, model output at six-hourly or shorter temporal resolutions, which is desirable for some applications such as urban drainage, is less widely available than daily output. Where limited studies have been undertaken, there is evidence that at the typical spatial resolutions used (i.e., non-cloud/convection-resolving scales), RCMs do not adequately represent sub-daily precipitation and the 
diurnal cycle of convection (Gutowski et al., 2003; Brockhaus et al., 2008; Lenderink and Van Meijgaard, 2008). Development of sub-daily statistical downscaling methods is constrained by the availability of long observed time series for calibration and validation and this approach is not currently widely used for climate change applications, although some weather generators, for example, do provide hourly information (Maraun et al., 2010).

It is not possible in this chapter to provide assessments of projected changes in extremes at spatial scales smaller than for large regions (Table 3-3). These large-region projections provide a wider context for national or more local projections, where they exist, and, where they do not, a first indication of expected changes, their associated uncertainties, and the evidence available. Several countries, for example in Europe, North America, Australia, and some other regions, have developed national or sub-national projections (generally based on dynamical and/or statistical downscaling), including information about extremes, and a range of other high-resolution information and tools are available from national weather and hydrological services and academic institutions to assist users and decisionmakers.

\subsubsection{Uncertainty Sources in Climate Change Projections}

Uncertainty in climate change projections arises at each of the steps involved in their preparation: determination of greenhouse gas and aerosol precursor emissions (driven by socioeconomic development and represented through the use of multiple emissions scenarios), concentrations of radiatively active species, radiative forcing, and climate response including downscaling. Also, uncertainty in the estimation of the true 'signal' of climate change is introduced by both errors in the model representation of Earth system processes and by internal climate variability.

As was noted in Section 3.2.3.1, most shortcomings in GCMs and RCMs result from the fact that many important small-scale processes (e.g., representations of clouds, convection, land surface processes) are not represented explicitly (Randall et al., 2007). Some processes particularly those involving feedbacks (Section 3.1.4), and this is especially the case for climate extremes and associated impacts - are still poorly represented and/or understood (e.g., land-atmosphere interactions, ocean-atmosphere interactions, stratospheric processes, blocking dynamics) despite some improvements in the simulations of others (see Box 3-2 and below). Therefore, limitations in computing power and in the scientific understanding of some physical processes currently restrict further global and regional climate model improvements. In addition, uncertainty due to structural or parameter errors in GCMs propagates directly from global model simulations as input to RCMs and thus to downscaled information.

These problems limit quantitative assessments of the magnitude and timing, as well as regional details, of some aspects of projected climate change. For instance, even atmospheric models with approximately $20-\mathrm{km}$ horizontal resolution still do not resolve the atmospheric processes sufficiently finely to simulate the high wind speeds and low pressure centers of the most intense hurricanes (Knutson et al., 2010). Realistically capturing details of such intense hurricanes, such as the inner eyewall structure, would require models with $1-\mathrm{km}$ horizontal resolution, far beyond the capabilities of current GCMs and of most current RCMs (and even global numerical weather prediction models). Extremes may also be impacted by mesoscale circulations that GCMs and even current RCMs cannot resolve, such as low-level jets and their coupling with intense precipitation (Anderson et al., 2003; Menendez et al., 2010). Another issue with small-scale processes is the lack of relevant observations, such as is the case with soil moisture and vegetation processes (Section 3.2.1) and relevant parameters (e.g., maps of soil types and associated properties, see for instance Seneviratne et al., 2006b; Anders and Rockel, 2009).

Since many extreme events, such as those associated with precipitation, occur at rather small temporal and spatial scales, where climate simulation skill is currently limited and local conditions are highly variable, projections of future changes cannot always be made with a high level of confidence (Easterling et al., 2008). The credibility of projections of changes in extremes varies with extreme type, season, and geographical region (Box 3-2). Confidence and credibility in projected changes in extremes increase when the physical mechanisms producing extremes in models are considered reliable, such as increases in specific humidity in the case of the projected increase in the proportion of summer precipitation falling as intense events in central Europe (Kendon et al., 2010). The ability of a model to capture the full distribution of variables - not just the mean - together with long-term trends in extremes, implies that some of the processes relevant to a future warming world may be captured (Alexander and Arblaster, 2009; van Oldenborgh et al., 2009). It should nonetheless be stressed that physical consistency of simulations with observed behavior provides necessary but not sufficient evidence for credible projections (Gutowski et al., 2008a).

While downscaling provides more spatial detail (Section 3.2.3.1), the added value of this step and the reliability of projections always needs to be assessed (Benestad et al., 2007; Laprise et al., 2008). A potential limitation and source of uncertainty in downscaling methods is that the calibration of statistical models and the parameterization schemes used in dynamical models are necessarily based on present (and past) climate (as well as an understanding of physical processes). Thus they may not be able to capture changes in extremes that are induced by future mechanistic changes in regional (or global) climate, that is, if used outside the range for which they were designed (Christensen et al., 2007). Spatial inhomogeneity of both land use/land cover and aerosol forcing adds to regional uncertainty. This means that the factors inducing uncertainty in the projections of extremes in different regions may differ considerably. Some specific issues inducing uncertainties in RCM projections are the interactions with the driving $\mathrm{GCM}$, especially in terms of biases and climate change signal (e.g., de Elía et al., 2008; Laprise et al., 2008; Kjellström and Lind, 2009; Déqué et al., 2011) and the choice of regional domain (Wang et al., 2004; Laprise et al., 2008). 
In the case of statistical downscaling, uncertainties are induced by, inter alia, the definition and choice of predictors (Benestad, 2001; Hewitson and Crane, 2006; Timbal et al., 2008) and the underlying assumption of stationarity (Raje and Mujumdar, 2010). In general, both approaches to downscaling are maturing and being more widely applied but are still restricted in terms of geographical coverage (Maraun et al., 2010). For many regions of the world, no downscaled information exists at all and regional projections rely only on information from GCMs (see Table 3-3).

For many user-driven applications, impact models need to be included as an additional step for projections (e.g., hydrological or ecosystem models). Because of the previously mentioned issues of scale discrepancies and overall biases, it is necessary to bias-correct RCM data before input to some impacts models (i.e., to bring the statistical properties of presentday simulations in line with observations and to use this information to correct projections). A number of bias correction methods, including quantile mapping and gamma transform, have recently been developed and exhibit promising skill for extremes of daily precipitation (Piani et al., 2010; Themeßl et al., 2011).

\subsubsection{Ways of Exploring and Quantifying Uncertainties}

Uncertainties can be explored, and quantified to some extent, through the combined use of observations and reanalyses, process understanding, a hierarchy of climate models, and ensemble simulations. Ensembles of model simulations represent a fundamental resource for studying the range of plausible climate responses to a given forcing (Meehl et al., 2007b; Randall et al., 2007). Such ensembles can be generated either by (i) collecting results from a range of models from different modelling centers (multi-model ensembles), to include the impact of structural model differences; (ii) by generating simulations with different initial conditions (intra-model ensembles) to characterize the uncertainties due to internal climate variability; or (iii) varying multiple internal model parameters within plausible ranges (perturbed and stochastic physics ensembles), with both (ii) and (iii) aiming to produce a more systematic estimate of single model uncertainty (Knutti et al., 2010b).

Many of the global models utilized for the AR4 were integrated as ensembles, permitting more robust statistical analysis than is possible if a model is only integrated to produce a single projection. Thus the available CMIP3 Multi-Model Ensemble (MME) GCM simulations reflect both interand intra-model variability. In advance of AR4, coordinated climate change experiments were undertaken which provided information from 23 models from around the world (Meehl et al., 2007a). The CMIP3 simulations were made available at the Program for Climate Model Diagnosis and Intercomparison (www-pcmdi.llnl.gov/ipcc/about_ipcc.php). However, the higher temporal resolution (i.e., daily) data necessary to analyze most extreme events were quite incomplete in the archive, with only four models providing daily averaged output with ensemble sizes greater than three realizations and many models not included at all. GCMs are expensive to run, thus a compromise is needed between the number of models, number of simulations, and the complexity of the models (Knutti, 2010).

Besides the uncertainty due to randomness itself, which is the canonical statistical definition, it is important to distinguish between the uncertainty due to insufficient agreement in the model projections, the uncertainty due to insufficient evidence (insufficient observational data to constrain the model projections or insufficient number of simulations from different models or insufficient understanding of the physical processes), and the uncertainty induced by insufficient literature, which refers to the lack of published analyses of projections. For instance, models may agree on a projected change, but if this change is controlled by processes that are not well understood and validated in the present climate, then there is an inherent uncertainty in the projections, no matter how good the model agreement may be. Similarly, available model projections may agree in a given change, but the number of available simulations may restrain the reliability of the inferred agreement (e.g., because the analyses need to be based on daily data that may not be available from all modelling groups). All these issues have been taken into account in assessing the confidence and likelihood of projected changes in extremes for this report (see Section 3.1.5).

Uncertainty analysis of the CMIP3 MME in AR4 focused essentially on the seasonal mean and inter-model standard deviation values (Christensen et al., 2007; Meehl et al., 2007b; Randall et al., 2007). In addition, confidence was assessed in the AR4 through simple quantification of the number of models that show agreement in the sign of a specific climate change (e.g., sign of the change in frequency of extremes) - assuming that the greater the number of models in agreement, the greater the robustness. However, the shortcoming of this definition of model agreement is that it does not take account of possible common biases among models. Indeed, the ensemble was strictly an 'ensemble of opportunity,' without sampling protocol, and the possible dependence of different models on one another (e.g., due to shared parameterizations) was not assessed (Knutti et al., 2010a). Furthermore, this particular metric, which assesses sign agreement only, can provide misleading conclusions in cases, for example, where the projected changes are near zero. For this reason, in our assessments of projected changes in extreme indices we consider the model agreement as a necessary but not a sufficient condition for likelihood statements [e.g., agreement of $66 \%$ of the models, as indicated with shading in several of the figures (Figures 3-3, 3-4, 3-6, 3-8, and $3-10)$, is a minimum but not a sufficient condition for a change being considered 'likely'].

Post-AR4 studies have concentrated more on the use of the MME in order to better characterize uncertainty in climate change projections, including those of extremes (Kharin et al., 2007; Gutowski et al., 2008a; Perkins et al., 2009). New techniques have been developed for exploiting the full ensemble information, in some cases using observational constraints to construct probability distributions (Tebaldi and Knutti, 2007; Tebaldi and Sanso, 2009), although issues such as determining appropriate metrics for weighting models are challenging (Knutti et al., 2010a). Perturbed-physics ensembles have also become available (e.g., 


\section{Box 3-2 | Variations in Confidence in Projections of Climate Change: Mean versus Extremes, Variables, Scale}

Comparisons of observed and simulated climate demonstrate good agreement for some climate variables such as mean temperature, especially at large horizontal scales (e.g., Räisänen, 2007). For instance, Figure 9.12 of the AR4 (Hegerl et al., 2007) compares the ability of 14 climate models to simulate the temporal variations of mean temperature through the 20th century. When the models included both natural and anthropogenic forcings, they consistently reproduced the decadal variations in global mean temperature. Without the anthropogenic influences the models consistently failed to reproduce the multi-decadal temperature variations. However, when the same models' abilities to simulate the temperature variations for smaller domains were assessed, although the mean temperature produced by the ensemble generally tracked the observed temperature changes, the consistency among the models was poorer than was the case for the global mean (Figure 9.12; Hegerl et al., 2007), partly because averaging over global scales smoothes internal variability or 'noise' more than averaging over smaller domains (see also Section 3.2.2.1). We can conclude that the smaller the spatial domain for which simulations or projections are being prepared, the less confidence we should have in these projections (although in some limited cases regional-scale projections can have higher reliability than larger-scale projections; see Section 3.1.6).

This increased uncertainty at smaller scales results from larger internal variability at smaller scales or 'noise' (i.e., natural variability unrelated to external forcings) and increased model uncertainty, both of which lead to lower model consistency at these scales (Hawkins and Sutton, 2009). The latter factor is largely due to the role of unresolved processes (representations of clouds, convection, land surface processes; see also Section 3.2.3). Hawkins and Sutton (2009) also point out regional variations in these aspects: in the tropics the temperature signal expected from anthropogenic factors is large relative to the model uncertainty and the natural variability, compared with higher latitudes. Figure 9.12 from AR4 (Hegerl et al., 2007) also shows that the models are more consistent in reproducing decadal temperature variations in the tropics than at higher latitudes, even though the magnitudes of the temperature trends are larger at higher latitudes.

Uncertainty in projections also depends on the variables, phenomena, or impacts considered (Sections 3.3. to 3.5.). There is more model uncertainty for variables other than temperature, for instance precipitation (Räisänen, 2007; Hawkins and Sutton, 2011; see also Section 3.2.3). And the situation is more difficult again for extremes. For instance, climate models simulate observed changes in extreme temperatures relatively well, but the frequency, distribution, and intensity of heavy precipitation is more poorly simulated (Randall et al., 2007) as are observed changes in heavy precipitation (e.g., Alexander and Arblaster, 2009). Also, projections of changes in temperature extremes tend to be more consistent across climate models (in terms of sign) than for (wet and dry) precipitation extremes (Tebaldi et al., 2006; Orlowsky and Seneviratne, 2011; see also Figures 3-3 through 3-7 and 3-10) and significant inconsistencies are also found for projections of agricultural (soil moisture) droughts (Wang, 2005; see also Box 3-3; Figure 3-10). For some other extremes, such as tropical cyclones, differences in the regional-scale climate change projections between models can lead to marked differences in projected tropical cyclone activity associated with anthropogenic climate change (Knutson et al., 2010), and thus decrease confidence in projections of changes in that extreme.

The relative importance of various causes of uncertainties in projections is somewhat different for earlier compared with later future periods. For some variables (mean temperature, temperature extremes), the choice of emission scenario becomes more critical than model uncertainty for the second part of the 21st century (Tebaldi et al., 2006; Hawkins and Sutton, 2009, 2011) though this does not apply for mean precipitation and some precipitation-related extremes (Tebaldi et al., 2006; Hawkins and Sutton, 2009, 2011), and has in particular not been evaluated in detail for a wide range of extremes. Users need to be aware of such issues in deciding the range of uncertainties that is appropriate to consider for their particular risk or impacts assessment

In summary, confidence in climate change projections depends on the (temporal and spatial) scale and variable being considered and whether one considers extremes or mean quantities. Confidence is highest for temperature, especially at the global scale, and decreases when other variables are considered, and when we focus on smaller spatial domains (Tables 3-1 and 3-3). Confidence in projections for extremes is generally weaker than for projections of long-term averages.

Collins et al., 2006; Murphy et al., 2007) and used to examine projected changes in extremes and their uncertainties (Barnett et al., 2006; Clark et al., 2006, 2010; Burke and Brown, 2008). Advances have also been made in developing probabilistic information at regional scales from the GCM simulations, but there has been rather less development extending this to probabilistic downscaled regional information and to 
extremes (Fowler et al., 2007a; Fowler and Ekstrom, 2009). Perhaps the most comprehensive approach to date for quantifying the influence of the cascade of uncertainties in regional projections is that used to develop the recent United Kingdom Climate Projections (UKCP09; Murphy et al., 2009). A complex Bayesian framework is used to combine a perturbed physics ensemble exploring uncertainties in atmosphere and ocean processes, and the carbon and sulfur cycles, with structural uncertainty (represented by 12 CMIP3 models) and an 11-member RCM perturbed physics ensemble. The published projections provide probability distributions of changes in various parameters including the wettest and hottest days of each season for $25-\mathrm{km}$ grid squares across the United Kingdom. These probabilities are conditional on the emissions scenario (low, medium, high) and are described as representing the "relative degree to which each climate outcome is supported by the evidence currently available, taking into account our understanding of climate science and observations, and using expert judgment" (Murphy et al., 2009).

Both statistical and dynamical downscaling methods are affected by the uncertainties that affect the global models, and a further level of uncertainty associated with the downscaling step also needs to be taken into consideration (see also Sections 3.2.3.1 and 3.2.3.2). The increasing availability of coordinated RCM simulations for different regions permits more systematic exploration of dynamical downscaling uncertainty. Such simulations are available for Europe (e.g., Christensen and Christensen, 2007; van der Linden and Mitchell, 2009) and a few other regions such as North America (Mearns et al., 2009) and West Africa (van der Linden and Mitchell, 2009; Hourdin et al., 2010). RCM intercomparisons have also been undertaken for a number of regions including Asia (Fu et al., 2005), South America (Menendez et al., 2010) and the Arctic (Inoue et al., 2006). A new series of coordinated simulations covering the globe is planned (Giorgi et al., 2009). Increasingly, RCM output from coordinated simulations is made available at the daily time scale, facilitating the analysis of some extreme events. Nevertheless, it is important to point out that ensemble runs with RCMs currently involve a limited number of driving GCMs, and hence only subsample uncertainty space. Ensuring adequate sampling of RCM simulations (both in terms of the number of considered RCMs and number of considered driving GCMs) may be more important for extremes than for changes in mean values (Frei et al., 2006; Fowler et al., 2007a). Internal variability, for example, has been shown to make a significant contribution to the spectrum of variability on at least multi-annual time scales and potentially up to multi-decadal time scales (Kendon et al., 2008; Hawkins and Sutton, 2009, 2011; Box 3-2).

\subsection{Observed and Projected Changes in Weather and Climate Extremes}

\subsubsection{Temperature}

Temperature is associated with several types of extremes, for example, heat waves and cold spells, and related impacts, for example, on human health, the physical environment, ecosystems, and energy consumption (e.g., Chapter 4, Sections 3.5.6 and 3.5.7; see also Case Studies 9.2.1 and 9.2.10). Temperature extremes often occur on weather time scales that require daily or higher time scale resolution data to accurately assess possible changes (Section 3.2.1). It is important to distinguish between daily mean, maximum (i.e., daytime), and minimum (nighttime) temperature, as well as between cold and warm extremes, due to their differing impacts. Spell lengths (e.g., duration of heat waves) are relevant for a number of impacts. Note that we do not consider here changes in diurnal temperature range or frost days, which are not typical 'climate extremes'. There is an extensive body of literature regarding the mechanisms of changes in temperature extremes (e.g., Christensen et al., 2007; Meehl et al., 2007b; Trenberth et al., 2007). Heat waves are generally caused by quasi-stationary anticyclonic circulation anomalies or atmospheric blocking (Xoplaki et al., 2003; Meehl and Tebaldi, 2004; Cassou et al., 2005; Della-Marta et al., 2007b), and/or land-atmosphere feedbacks (in transitional climate regions), whereby the latter can act as an amplifying mechanism through reduction in evaporative cooling (Section 3.1.4), but also induce enhanced persistence due to soil moisture memory (Lorenz et al., 2010). Also snow feedbacks (Section 3.1.4), and possibly changes in aerosols (Portmann et al., 2009), are relevant for temperature extremes. Trends in temperature extremes (either observed or projected) can sometimes be different for the most extreme temperatures (e.g., annual maximum/minimum daily maximum/minimum temperature) than for less extreme events [e.g., cold/warm days/nights; see, for instance, Brown et al. (2008) versus Alexander et al. (2006)]. One reason for this is that 'moderate extremes' such as warm/cold days/nights are generally computed for each day with respect to the long-term statistics for that day, thus, for example, an increase in warm days for annual analyses does not necessarily imply warming for the very warmest days of the year.

\section{Observed Changes}

Regional historical or paleoclimatic temperature reconstructions may help place the recent instrumentally observed temperature extremes in the context of a much longer period, but literature on this topic is very sparse and most regional reconstructions are for Europe. For example Dobrovolny et al. (2010) reconstructed monthly and seasonal temperature over central Europe back to 1500 using a variety of temperature proxy records. They concluded that the summer 2003 heat wave and the July 2006 heat wave exceeded the +2 standard deviation (associated with the reconstruction method) of previous monthly temperature extremes since 1500. Barriopedro et al. (2011) showed that the anomalously warm summers of 2003 in western and central Europe and 2010 in eastern Europe and Russia both broke the 500-year long seasonal temperature record over $50 \%$ of Europe. The coldest periods within the last five centuries occurred in the winter and spring of 1690 . Another 500-year temperature reconstruction was recently completed for the Mediterranean basin by means of documentary data and instrumental observations (Camuffo et al., 2010). It suggests strong natural variability in the basin, possibly exceeding the recent warming, although discontinuities in the records limit the interpretation of this finding. 
The AR4 (Trenberth et al., 2007, based on Alexander et al., 2006) reported a statistically significant increase in the numbers of warm nights and a statistically significant reduction in the numbers of cold nights for 70 to $75 \%$ of the land regions with data (for the spatial coverage of the underlying data set and the definition of warm/cold days and nights, see Section 3.2.1 and Box 3-1, respectively). Changes in the numbers of warm days and cold days also showed warming, but less marked than for nights, with about 40 to $50 \%$ of the area with data showing statistically significant changes consistent with warming (Alexander et al., 2006). Less than $1 \%$ of the area with data showed statistically significant trends in cold/warm days and nights that were consistent with cooling (Alexander et al., 2006). Trenberth et al. (2007) also reported, based on Vose et al. (2005), that from 1950 to 2004, the annual trends in minimum and maximum land-surface air temperature averaged over regions with data were $0.20^{\circ} \mathrm{C}$ per decade and $0.14^{\circ} \mathrm{C}$ per decade, respectively, and that for 1979 to 2004 , the corresponding linear trends for the land areas with data were $0.29^{\circ} \mathrm{C}$ per decade for both maximum and minimum temperature. Based on this evidence, the IPCC AR4 (SPM; IPCC, 2007b) assessed that it was very likely that there had been trends toward warmer and more frequent warm days and warm nights, and warmer and less frequent cold days and cold nights in most land areas.

Regions that were found to depart from this overall behavior toward more warm days and nights and fewer cold days and nights in Alexander et al. (2006) were mostly central North America, the eastern United States, southern Greenland (increase in cold days and decreases in warm days), and the southern half of South America (decrease in warm days; no data available for the northern half of the continent). In central North America and the eastern United States this partial tendency for a negative trend in extremes is also consistent with a reported mean negative trend in temperatures, mostly in the spring to summer season (also termed 'warming hole', e.g., Pan et al., 2004; Portmann et al., 2009). Several explanations have been suggested for this behavior, which seems partly associated with a change in the hydrological cycle, possibly linked to soil moisture and/or aerosol feedbacks (Pan et al., 2004; Portmann et al., 2009).

More recent analyses available since the AR4 include a global study (for annual extremes) by Brown et al. (2008) based on the data set from Caesar et al. (2006), and regional studies for North America (Peterson et al., 2008a; Meehl et al., 2009c), Central-Western Europe (since 1880; Della-Marta et al., 2007a), central and eastern Europe (Bartholy and Pongracz, 2007; Kürbis et al., 2009), the eastern Mediterranean region including Turkey (Kuglitsch et al., 2010), western Central Africa, Guinea Conakry and Zimbabwe (Aguilar et al., 2009), the Tibetan Plateau (You et al., 2008) and China (You et al., 2011), Uruguay (Rusticucci and Renom, 2008), and Australia (Alexander and Arblaster, 2009). Further references can also be found in Table 3-2. Overall, these studies are consistent with the assessment of an increase in warm days and nights and a reduction in cold days and nights on the global basis, although they do not necessarily consider trends in all four variables, and a few single studies report trends that are not statistically significant or even trends opposite to the global tendencies in some extremes, subregions, seasons, or decades. For instance, Rusticucci and Renom (2008) found in Uruguay a reduction of cold nights, a positive but a statistically insignificant trend in warm nights, statistically insignificant decreases in cold days at most investigated stations, and inconsistent trends in warm days. Together with the previous results from Alexander et al. (2006) for southern South America (see above) and further regional studies (Table 3-2), this suggests a less consistent warming tendency in South America compared to other continents. Another notable feature is that studies for central and southeastern Europe display a marked change point in trends in temperature extremes at the end of the 1970 s/beginning of 1980s (Table 3-2), which for some extremes can lead to very small and/or statistically not significant overall trends since the 1960s (e.g., Bartholy and Pongracz, 2007).

There are fewer studies available investigating changes in characteristics of cold spells and warm spells, or cold waves and heat waves, compared with studies of the intensity or frequency of warm and cold days or nights. Alexander et al. (2006) provided an analysis of trends in warm spells [based on the Warm Spell Duration Index (WSDI); see Table 3-2 and Box 3-1] mostly in the mid- and high-latitudes of the Northern Hemisphere. The analysis displays a tendency toward a higher length or number of warm spells (increase in number of days belonging to warm spells) in much of the region, with the exception of the southeastern United States and eastern Canada. Regional studies on trends in warm spells or heat waves are also listed in Table 3-2. Kunkel et al. (2008) found that the United States has experienced a general decline in cold waves over the 20th century, with a spike of more cold waves in the 1980s. Further, they report a strong increase in heat waves since 1960, although the heat waves of the 1930s associated with extreme drought conditions still dominate the 1895-2005 time series. Kuglitsch et al (2009) reported an increase in heat wave intensity, number, and length in summer over the 1960-2006 time period in the eastern Mediterranean region. Ding et al. (2010) reported increasing numbers of heat waves over most of China for the 1961-2007 period. The record-breaking heat wave over western and central Europe in the summer of 2003 is an example of an exceptional recent extreme (Beniston, 2004; Schär and Jendritzky, 2004). That summer (June to August) was the hottest since comparable instrumental records began around 1780 and perhaps the hottest since at least 1500 (Luterbacher et al., 2004). Other examples of recent extreme heat waves include the 2006 heat wave in Europe (Rebetez et al., 2008), the 2007 heat wave in southeastern Europe (Founda and Giannakopoulos, 2009), the 2009 heat wave in southeastern Australia (National Climate Centre, 2009), and the 2010 heat wave in Russia (Barriopedro et al., 2011). Both the 2003 European heat wave (Andersen et al., 2005; Ciais et al., 2005) and the 2009 southeastern Australian heat wave were also associated with drought conditions, which can strongly enhance temperature extremes during heat waves in some regions (see also Section 3.1.4).

Some recent analyses have led to revisions of previously reported trends. For instance, Della-Marta et al. (2007a) found that mean summer maximum temperature change over Europe was $+1.6 \pm 0.4^{\circ} \mathrm{C}$ during 1880 to 2005 , a somewhat greater increase than reported in earlier 


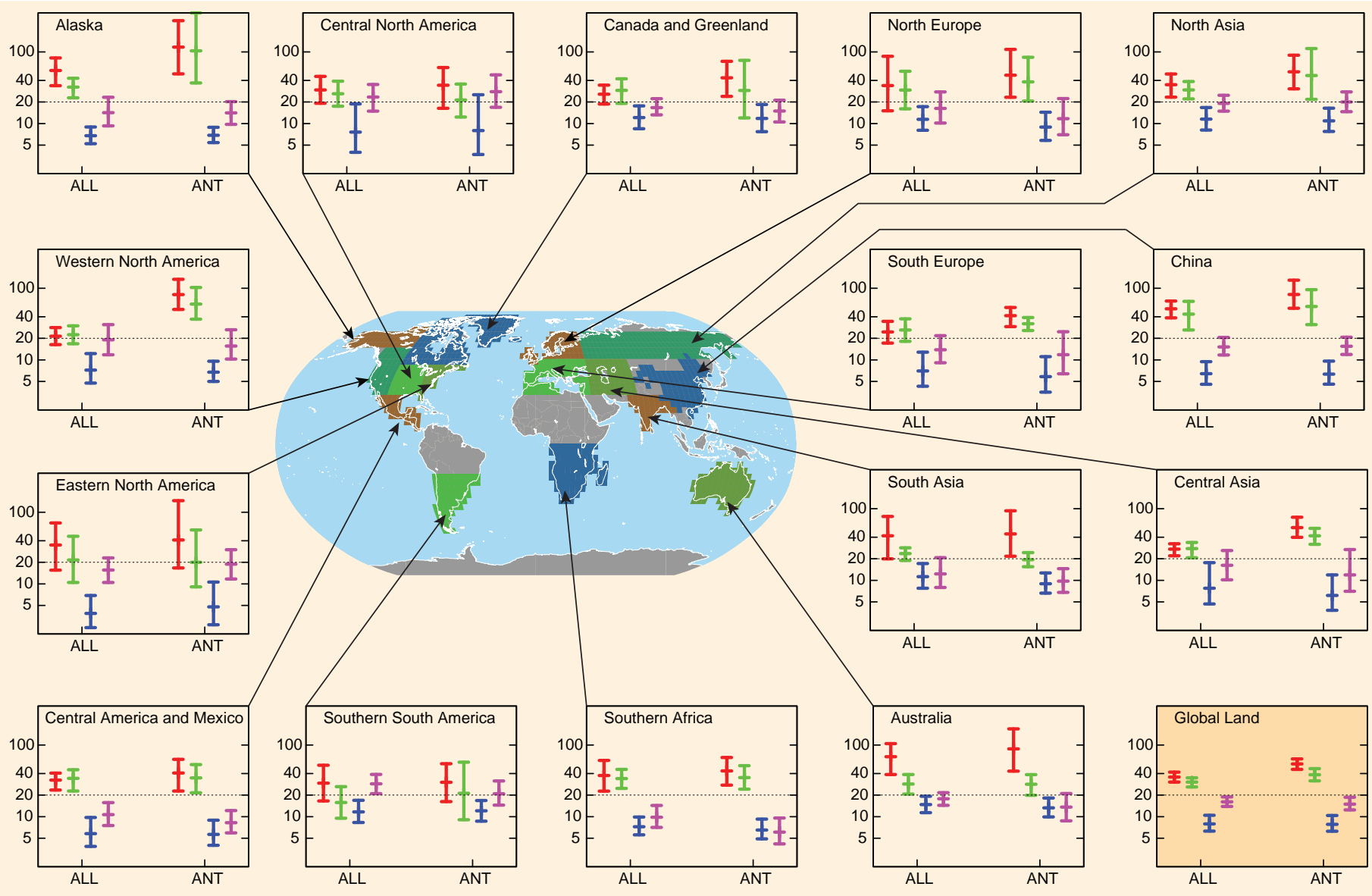

Figure 3-2 | Estimated return periods (years) and their 5 and 95\% uncertainty limits for 1960s 20-year return values of annual extreme daily temperatures in the 1990s climate (see text for more details). ANT refers to model simulated responses with only anthropogenic forcing and ALL is both natural and anthropogenic forcing. Error bars are for annual minimum daily minimum temperature (red: TNn), annual minimum daily maximum temperature (green: TXn), annual maximum daily minimum temperature (blue: TNx), and annual maximum daily maximum temperature (pink: TXx), respectively. Grey areas have insufficient data. Source: Zwiers et al., (2011).

studies. Kuglitsch et al. $(2009,2010)$ homogenized and analyzed over 250 daily maximum and minimum temperature series in the Mediterranean region since 1960, and found that after homogenization the positive trends in the frequency of hot days and heat waves in the Eastern Mediterranean region were higher than reported in earlier studies. This was due to the correction of many warm-biased temperature data in the region during the 1960s and 1970s.

In summary, regional and global analyses of temperature extremes on land generally show recent changes consistent with a warming climate at the global scale, in agreement with the previous assessment in AR4. Only a few regions show changes in temperature extremes consistent with cooling, most notably for some extremes in central North America, the eastern United States, and also parts of South America. Based on the available evidence we conclude that it is very likely that there has been an overall decrease in the number of cold days and nights and very likely that there has been an overall increase in the number of warm days and nights in most regions, that is, for land areas with data (corresponding to about 70 to $80 \%$ of all land areas; see Table 3-2). It is likely that this statement applies at the continental scale in North America, Europe, and Australia (Table 3-2). However, some subregions on these continents have had warming trends in temperature extremes that were small or not statistically significant (e.g., southeastern Europe), and a few subregions have had cooling trends in some temperature extremes (e.g., central North America and eastern United States). Asia also shows trends consistent with warming in most of the continent, but which are assessed here to be of medium confidence because of lack of literature for several regions apart from the global study from Alexander et al. (2006). Most of Africa is insufficiently well sampled to allow an overall likelihood statement to be made at the continental scale, although most of the regions on this continent for which data are available have exhibited warming in temperature extremes (Table 3-2). In South America, both lack of data and some inconsistencies in the reported trends imply low confidence in the overall trends at the continental scale (Table 3-2). In many (but not all) regions with sufficient data there is medium confidence that the number of warm spells or heat waves has increased since the middle of the 20th century (Table 3-2).

\section{Causes of Observed Changes}

The AR4 (Hegerl et al., 2007) concluded that surface temperature extremes have likely been affected by anthropogenic forcing. This assessment was based on multiple lines of evidence of temperature 
extremes at the global scale including the reported increase in the number of warm extremes and decrease in the number of cold extremes at that scale (Alexander et al., 2006). Hegerl et al. (2007) also state that anthropogenic forcing may have substantially increased the risk of extreme temperatures (Christidis et al., 2005) and of the 2003 European heat wave (Stott et al., 2004).

Recent studies on attribution of changes in temperature extremes have tended to reaffirm the conclusions reached in the AR4. Alexander and Arblaster (2009) found that trends in warm nights over Australia could only be reproduced by a coupled model that included anthropogenic forcings. As part of the recent report of the US Climate Change Science Program (CCSP, 2008), Gutowski et al. (2008a) concluded that most of the observed changes in temperature extremes for the second half of the 20th century over the United States can be attributed to human activity. They compared observed changes in the number of frost days, the length of growing season, the number of warm nights, and the heat wave intensity with those simulated in a nine-member multi-model ensemble simulation. The decrease in frost days, an increase in growing season length, and an increase in heat wave intensity all show similar changes over the United States in 20th-century experiments that combine anthropogenic and natural forcings, though the relative contributions of each are unclear.

Results from two global coupled climate models with separate anthropogenic and natural forcing runs indicate that the observed changes are simulated with anthropogenic forcings, but not with natural forcings (even though there are some differences in the details of the forcings). Zwiers et al. (2011) compared observed annual temperature extremes including annual maximum daily maximum and minimum temperatures, and annual minimum daily maximum and minimum temperatures with those simulated responses to anthropogenic forcing or anthropogenic and natural external forcings combined by multiple GCMs. They fitted probability distributions (Box 3-1) to the observed extreme temperatures with a time-evolving pattern of location parameters as obtained from the model simulations, and found that both anthropogenic influence and the combined influence of anthropogenic and natural forcing can be detected in all four extreme temperature variables at the global scale over the land, and also over many large land areas. Globally, return periods for events that were expected to recur once every 20 years in the 1960s are now estimated to exceed 30 years for extreme annual minimum daily maximum temperature and 35 years for extreme annual minimum daily minimum temperature, although these estimates are subject to considerable uncertainty. Further, return periods were found to have decreased to less than 10 or 15 years for annual maximum daily minimum and daily maximum temperatures respectively (Figure 3-2).

However, the available detection and attribution studies for extreme maximum and minimum temperatures (Christidis et al., 2011b; Zwiers et al., 2011) suggest that the models overestimate changes in the maximum temperatures and underestimate changes in the minimum temperatures during the late 20th century.

\section{Projected Changes and Uncertainties}

Regarding projections of extreme temperatures, the AR4 (Meehl et al., 2007b) noted that cold episodes were projected to decrease significantly in a future warmer climate and considered it very likely that heat waves would be more intense, more frequent, and last longer in a future warmer climate. Post-AR4 studies of temperature extremes have utilized larger model ensembles (Kharin et al., 2007; Sterl et al., 2008; Orlowsky and Seneviratne, 2011) and generally confirm the conclusions of the AR4, while also providing more specific assessments both in terms of the range of considered extremes and the level of regional detail (see also Table 3-3).

There are few global analyses of multi-model projections of temperature extremes available in the literature. The study by Tebaldi et al. (2006), which provided the basis for extreme projections given in the AR4 (Figures 10.18 and 10.19 in Meehl et al., 2007b), provided global analyses of projected changes (A1B scenario) in several extremes indices based on nine GCMs (note that not all modelling groups that saved daily data also calculated the indices). For temperature extremes, analyses were provided for heat wave lengths (using only one index, see discussion in Box 3-1) and warm nights. Stippling was used where five out of nine models displayed statistically significant changes of the same sign. Orlowsky and Seneviratne (2011) recently updated the analysis from Tebaldi et al. (2006) for the full ensemble of GCMs that contributed A2 scenarios to the CMIP3, using a larger number of extreme indices [including several additional analyses of daily extremes (see Figures 3-3 and 3-4), and three heat wave indices instead of one; see also discussion of heat wave indices in Box 3-1], using other thresholds for display and stippling of the figures (no results displayed if less than $66 \%$ of the models agree on the sign of change; stippling used only for $90 \%$ model agreement), and providing seasonal analyses. This analysis confirms that strong agreement (in terms of sign of change) exists between the various GCM projections for temperature-related extremes, with projected increases in warm day occurrences (Figure 3-3) and heat wave length, and decreases in cold extremes (Figure 3-4). Temperature extremes on land are projected to warm faster than global annual mean temperature in many regions and seasons, implying large changes in extremes in some places, even for a global warming of 2 or $3^{\circ} \mathrm{C}$ (with scaling factors for the SRES A2 scenario ranging between 0.5 and 2 for moderate seasonal extremes; Orlowsky and Seneviratne, 2011). Based on the analyses of Tebaldi et al. (2006) and Orlowsky and Seneviratne (2011), as well as physical considerations, we assess that increases in the number of warm days and nights and decreases in the number of cold days and nights (defined with respect to present regional climate, i.e., the 1961-1990 reference period, see Box 3-1) are virtually certain at the global scale. Further, given the assessed changes in hot and cold days and nights and available analyses of projected changes in heat wave length in the two studies, we assess that it is very likely that the length, frequency, and/or intensity of heat waves will increase over most land areas.

Another global study of changes in extremes based on the CMIP3 ensemble is provided in Kharin et al. (2007), which focuses on changes 
in annual extremes (20-year extreme values) based on 12 GCMs for temperature extremes and $14 \mathrm{GCMs}$ for precipitation extremes employing the SRES A2, A1B, and B1 emissions scenarios. This analysis projects increases in the temperature of the 1-in-20 year annual extreme hottest day of about 2 to $6^{\circ} \mathrm{C}$ (depending on region and scenario; Figure 3-5 adapted from Kharin et al., 2007) and strong reductions in the return periods of this extreme event by the end of the 21st century. However, as noted above, the limited number of relevant detection and attribution studies suggests that models may overestimate some changes in temperature extremes, and our assessments take this into account by reducing the level of certainty in the assessments from what would be derived by uncritical acceptance of the projections in Figure 3-5. The assessments are also weakened to reflect the possibility that some important processes relevant to extremes may be missing or be poorly represented in models, as well as the fact that the model projections considered in this study did not correspond to the full CMIP3 ensemble. Hence, we assess that in terms of absolute values, the 20-year extreme annual daily maximum temperature (i.e., return value) will likely increase by about 2 to $5^{\circ} \mathrm{C}$ by the late 21 st century, and by about 1 to $3^{\circ} \mathrm{C}$ by mid-21st century, depending on the region and emissions scenario (considering the B1, A1B, and A2 scenarios; Figure 3-5a). Furthermore, we assess that globally under the $\mathrm{A} 2$ and $\mathrm{A} 1 \mathrm{~B}$ scenarios a 1 -in-20 year annual extreme hot day is likely to become a 1-in-2 year annual extreme by the end of the 21st century in most regions, except in the high latitudes of the Northern Hemisphere where it is likely to become a 1 -in-5 year annual extreme (Figure 3-5b, based on material from Kharin et al., 2007). Further, we assess that under the more moderate $B 1$ scenario a current 1-in-20 year extreme would likely become a 1-in-5 year event (and a 1-in-10 year event in Northern Hemisphere high latitudes).

Next, regional assessments of projected changes in temperature extremes are provided. More details are found in Table 3-3. For North America, the CCSP reached the following conclusions (using IPCC AR4 likelihood terminology) regarding projected changes in temperature extremes by the end of the 21st century (Gutowski et al., 2008a):

1) Abnormally hot days and warm nights and heat waves are very likely to become more frequent.

2) Cold days and cold nights are very likely to become much less frequent.

3) For a mid-range scenario (A1B) of future greenhouse gas emissions, a day so hot that it is currently experienced only once every 20 years would occur every 3 years by the middle of the century over
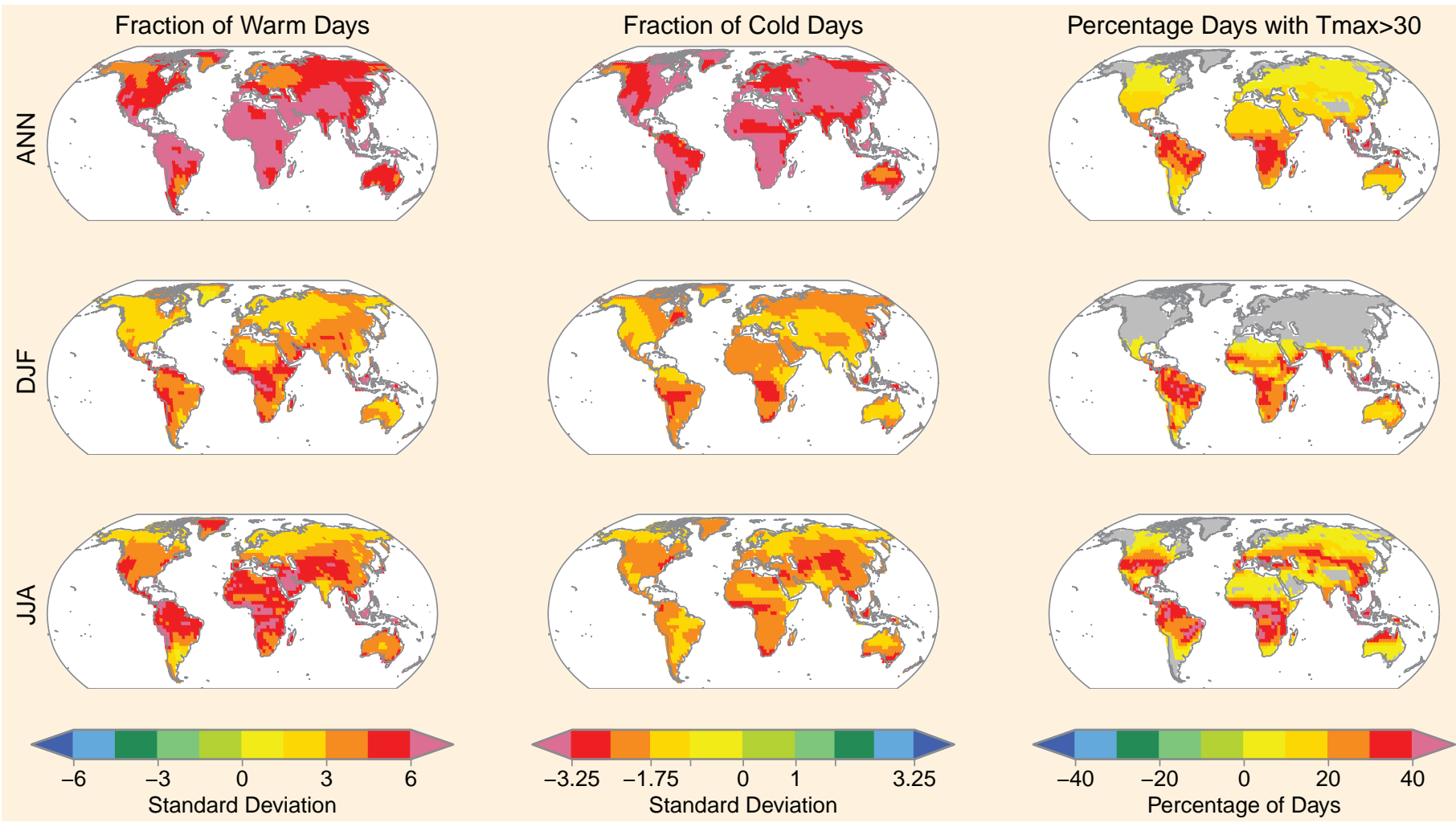

Figure 3-3 | Projected annual and seasonal changes in three indices for daily Tmax for 2081-2100 with respect to 1980-1999, based on 14 GCMs contributing to the CMIP3. Left column: fraction of warm days (days in which Tmax exceeds the 90th percentile of that day of the year, calculated from the 1961-1990 reference period); middle column: fraction of cold days (days in which Tmax is lower than the 10th percentile of that day of the year, calculated from the 1961-1990 reference period); right column: percentage of days with $\operatorname{Tmax}>30^{\circ} \mathrm{C}$. The changes are computed for the annual time scale (top row) and two seasons (December-January-February, DJF, middle row, and June-July-August, JJA, bottom row) as the fractions/percentages in the 2081-2100 period (based on simulations for emission scenario SRES A2) minus the fractions/percentages of the 1980-1999 period (from corresponding simulations for the 20th century). Warm day and cold day changes are expressed in units of standard deviations, derived from detrended per year annual or seasonal estimates, respectively, from the three 20-year periods 1980-1999, 2046-2065, and 2081-2100 pooled together. Tmax $>30^{\circ} \mathrm{C}$ changes are given directly as differences in percentage points. Color shading is only applied for areas where at least $66 \%$ (i.e., 10 out of 14) of the GCMs agree on the sign of the change; stippling is applied for regions where at least $90 \%$ (i.e.,13 out of 14) of the GCMs agree on the sign of the change. Adapted from Orlowsky and Seneviratne (2011); updating Tebaldi et al. (2006) for additional number of indices and CMIP3 models, and including seasonal time frames. For more details, see Appendix 3.A. 

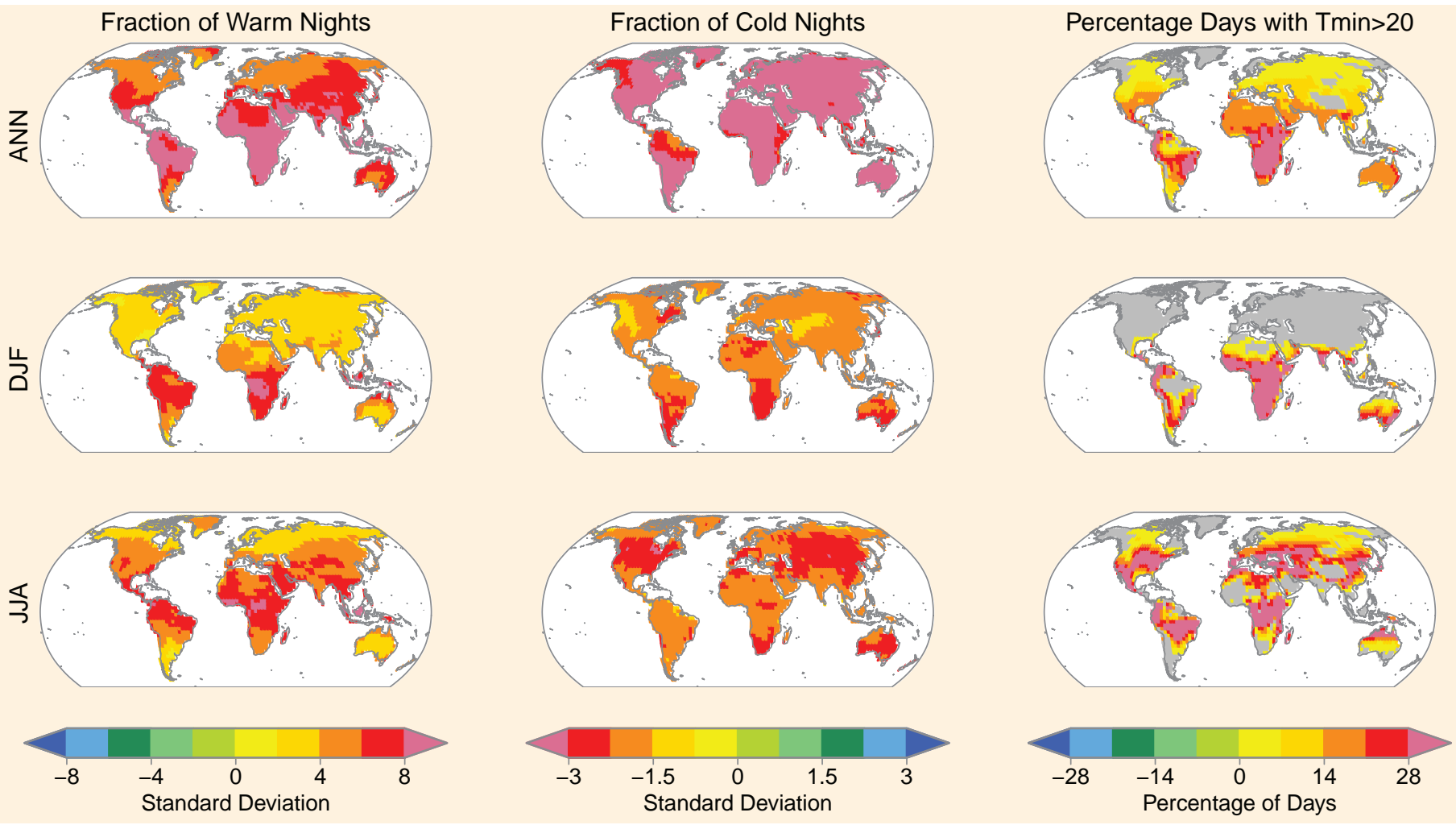

Figure 3-4 | Projected annual and seasonal changes in three indices for daily Tmin for 2081-2100 with respect to 1980-1999, based on 14 GCMs contributing to the CMIP3. Left column: fraction of warm nights (days at which Tmin exceeds the 90th percentile of that day of the year, calculated from the 1961-1990 reference period); middle column: fraction of cold nights (days at which Tmin is lower than the 10th percentile of that day of the year, calculated from the 1961-1990 reference period); right column: percentage of days with Tmin $>20^{\circ} \mathrm{C}$. The changes are computed for the annual time scale (top row) and two seasons (December-January-February, DJF, middle row, and June-July-August, JJA, bottom row) as the fractions/percentages in the 2081-2100 period (based on simulations under emission scenario SRES A2) minus the fractions/percentages of the 1980-1999 period (from corresponding simulations for the 20th century). Warm night and cold night changes are expressed in units of standard deviations, derived from detrended per year annual or seasonal estimates, respectively, from the three 20-year periods 1980-1999, 2046-2065, and 2081-2100 pooled together. Tmin $>20^{\circ} \mathrm{C}$ changes are given directly as differences of percentage points. Color shading is only applied for areas where at least $66 \%$ (i.e., 10 out of 14) of the GCMs agree in the sign of the change; stippling is applied for regions where at least $90 \%$ (i.e., 13 out of 14) of the GCMs agree in the sign of the change. Adapted from Orlowsky and Seneviratne (2011); updating Tebaldi et al. (2006) for additional number of indices and CMIP3 models, and including seasonal time frames. For more details, see Appendix 3.A.

much of the continental United States and every 5 years over most of Canada; by the end of the century, it would occur every other year or more.

Meehl et al. (2009c) examined changes in record daily high and low temperatures in the United States and show that even with projected strong warming resulting in many more record highs than lows, the occasional record low is still set. For Australia, the CMIP3 ensemble projected increases in warm nights $(15-40 \%$ by the end of the 21 st century) and heat wave duration, together with a decrease in the number of frost days (Alexander and Arblaster, 2009). Inland regions show greater warming compared with coastal zones (Suppiah et al., 2007; Alexander and Arblaster, 2009) and large increases in the number of days above 35 or $40^{\circ} \mathrm{C}$ are indicated (Suppiah et al., 2007). For the entire South American region, a study with a single RCM projected more frequent warm nights and fewer cold nights (Marengo et al., 2009a). Several studies of regional and global model projections of changes in extremes are available for the European continent (see also Table 3-3). Analyses of both global and regional model outputs show major increases in warm temperature extremes across the Mediterranean region including events such as hot days $\left(\operatorname{Tmax}>30^{\circ} \mathrm{C}\right.$ ) and tropical nights (Tmin $>20^{\circ} \mathrm{C}$ ) (Giannakopoulos et al., 2009; Tolika et al., 2009).
Comparison of RCM projections using the A1B forcing scenario, with data for 2007 (the hottest summer in Greece in the instrumental record with a record daily Tmax observed value of $44.8^{\circ} \mathrm{C}$ ) indicates that the distribution for 2007 is closer to the distribution for 2071-2100 than for the 2021-2050 period, thus 2007 might be considered a 'normal' summer of the future (Founda and Giannakopoulos, 2009; Tolika et al., 2009). Beniston et al. (2007) concluded from an analysis of RCM output that regions such as France and Hungary may experience as many days per year above $30^{\circ} \mathrm{C}$ as currently experienced in Spain and Sicily. In this RCM ensemble, France was the area with the largest projected warming in the uppermost percentiles of daily summer temperatures although the mean warming was greatest in the Mediterranean region (Fischer and Schär, 2009). New results from an RCM ensemble project increases in the amplitude, frequency, and duration of health-impacting heat waves, especially in southern Europe (Fischer and Schär, 2010). Overall these regional assessments are consistent with the global assessments provided above. It should be noted, however, that the assessed uncertainty is larger at the regional level than at the continental or global level (see Box 3-2). Global-scale trends in a specific extreme may be either more reliable or less reliable than regional-scale trends, depending on the geographical uniformity of the trends in the specific extreme (Section 3.1.6). 


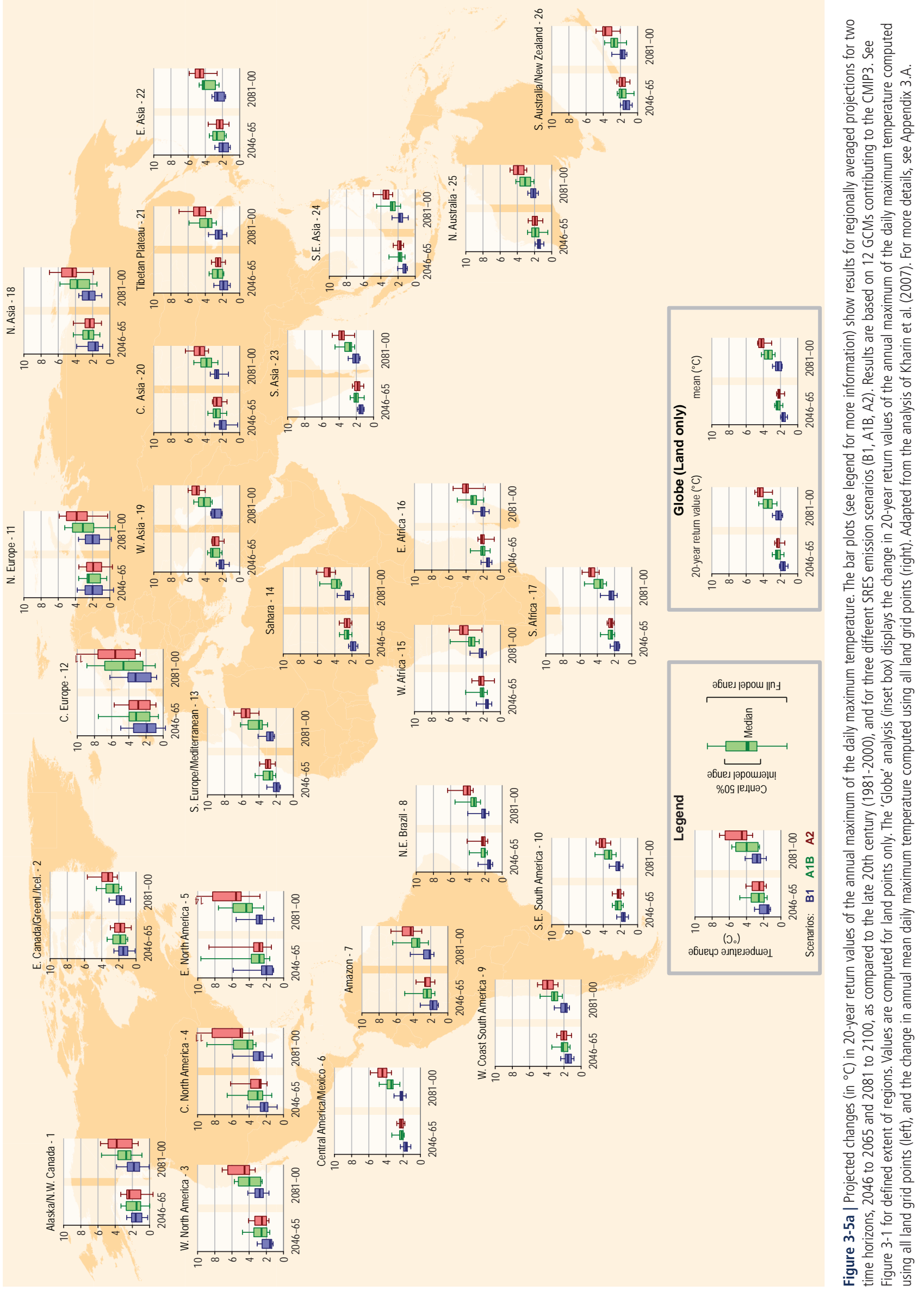







Temperature extremes were the type of extremes projected to change with most confidence in the AR4 (IPCC, 2007a). This is confirmed regarding the sign of change with more recent analyses (Figures 3-3 and 3-4), although there is a large spread with respect to the magnitude of changes both due to emission scenario and climate model uncertainty (Figures 3-5a,b). If changes in temperature extremes scale with changes in mean temperature (i.e., simple shifts of the probability distribution), we infer that it is virtually certain that hot extremes will increase and cold extremes will decrease over the 21st century with respect to the 1960-1990 climate. Changes in the tails of the temperature distributions may not scale with changes in the mean in some regions (Section 3.1.6), though in most such reported cases hot extremes tend to increase and cold extremes decrease more than mean temperature, and thus the above statement for extremes (virtually certain increase in hot extremes and decrease in cold extremes) still applies. Central and eastern Europe is a region where the evidence suggests that projected changes in temperature extremes result from both changes in the mean as well as from changes in the shape of the probability distributions (Schär et al., 2004). The main mechanism for the widening of the distribution is linked to the drying of the soil in this region (Sections 3.1.4 and 3.1.6). Furthermore, remote surface heating may induce circulation changes that modify the temperature distribution (Haarsma et al., 2009). Other local, mesoscale, and regional feedback mechanisms, in particular with land surface conditions (beside soil moisture, also with vegetation and snow; Section 3.1.4) and aerosol concentrations (Ruckstuhl and Norris, 2009) may enhance the uncertainties in temperature projections. Some of these processes occur at a small scale unresolved by the models (Section 3.2.3). In addition, lack of observational data (e.g., for soil moisture and snow cover; see Section 3.2.1) reduces the possibilities to evaluate climate models (e.g., Roesch, 2006; Boe and Terray, 2008; Hall et al., 2008; Brown and Mote, 2009). Because of these various processes and associated uncertainties, mean global warming does not necessarily imply warming in all regions and seasons (see also Section 3.1.6). Regarding mesoscale processes, lack of information also affects confidence in projections. One example is changes in heat waves in the Mediterranean region that are suggested to have the largest impact in coastal areas, due to the role of enhanced relative humidity in health impacts (Diffenbaugh et al., 2007; Fischer and Schär, 2010). But it is not clear how this pattern may or may not be moderated by sea breezes (Diffenbaugh et al., 2007).

In summary, since 1950 it is very likely that there has been an overall decrease in the number of cold days and nights and an overall increase in the number of warm days and nights at the global scale, that is, for land areas with sufficient data. It is likely that such changes have also occurred at the continental scale in North America, Europe, and Australia. There is medium confidence in a warming trend in daily temperature extremes in much of Asia. Confidence in historical trends in daily temperature extremes in Africa and South America generally varies from low to medium depending on the region. Globally, in many (but not all) regions with sufficient data there is medium confidence that the length or number of warm spells or heat waves has increased since the middle of the 20th century. It is likely that anthropogenic influences have led to warming of extreme daily minimum and maximum temperatures at the global scale. Models project substantial warming in temperature extremes by the end of the 21st century. It is virtually certain that increases in the frequency and magnitude of warm days and nights and decreases in the cold days and nights will occur through the 21st century at the global scale. This is mostly linked with mean changes in temperatures, although changes in temperature variability can play an important role in some regions. It is very likely that the length, frequency, and/or intensity of warm spells or heat waves (defined with respect to present regional climate) will increase over most land areas. For the SRES A2 and A1B emission scenarios a 1-in-20 year annual hottest day is likely to become a 1-in-2 year annual extreme by the end of the 21st century in most regions, except in the high latitudes of the Northern Hemisphere where it is likely to become a 1-in-5 year annual extreme. In terms of absolute values, 20-year extreme annual daily maximum temperature (i.e., return value) will likely increase by about 1 to $3^{\circ} \mathrm{C}$ by mid-21st century and by about 2 to $5^{\circ} \mathrm{C}$ by the late 21st century, depending on the region and emissions scenario (Figure 3-5). Moderate temperature extremes on land are projected to warm faster than global annual mean temperature in many regions and seasons. Projected changes at subcontinental scales are less certain than is the case for the global scale. Regional changes in temperature extremes will differ from the mean global temperature change. Mean global warming does not necessarily imply warming in all regions and seasons.

\subsubsection{Precipitation}

This section addresses changes in daily extreme or heavy precipitation events. Reductions in mean (or total) precipitation that can lead to drought (i.e., associated with lack of precipitation) are considered in Section 3.5.1. Because climates are so diverse across different parts of the world, it is difficult to provide a single definition of extreme or heavy precipitation. In general, two different approaches have been used: (1) relative thresholds such as percentiles (typically the 95th percentile) and return values; and (2) absolute thresholds [e.g., $50.8 \mathrm{~mm}$ (2 inches) day $^{-1}$ of rain in the United States, and $100 \mathrm{~mm}$ day $^{-1}$ of rain in China]. For more details on the respective drawbacks and advantages of these two approaches, see Section 3.1 and Box 3-1. Note that we do not distinguish between rain and snowfall (both considered as contributors to overall extreme precipitation events) as they are not treated separately in the literature, but do distinguish changes in hail from other precipitation types. Increases in public awareness and changes in reporting practices have led to inconsistencies in the record of severe thunderstorms and hail that make it difficult to detect trends in the intensity or frequency of these events (Kunkel et al., 2008). Furthermore, weather events such as hail are not well captured by current monitoring systems and, in some parts of the world, the monitoring network is very sparse (Section 3.2.1), resulting in considerable uncertainty in the estimates of extreme 
precipitation. There are also known biases in precipitation measurements, mostly leading to rain undercatch. Little evidence of paleoclimatic and historical changes in heavy precipitation is available to place recent variations into context.

\section{Observed Changes}

The AR4 (Trenberth et al., 2007) concluded that it was likely that there had been increases in the number of heavy precipitation events (e.g., 95th percentile) over the second half of the 20th century within many land regions, even in those where there had been a reduction in total precipitation amount, consistent with a warming climate and observed significant increasing amounts of water vapor in the atmosphere. Increases had also been reported for rarer precipitation events (1-in-50 year return period), but only a few regions had sufficient data to assess such trends reliably. However, the AR4 (Trenberth et al., 2007) also stated that "Many analyses indicate that the evolution of rainfall statistics through the second half of the 20th century is dominated by variations on the interannual to inter-decadal time scale and that trend estimates are spatially incoherent (Manton et al., 2001; Peterson et al., 2002; Griffiths et al., 2003; Herath and Ratnayake, 2004)". Overall, as highlighted in Alexander et al. (2006), the observed changes in precipitation extremes were found at the time to be much less spatially coherent and statistically significant compared to observed changes in temperature extremes: although statistically significant trends toward stronger precipitation extremes were generally found for a larger fraction of the land area than trends toward weaker precipitation extremes, statistically significant changes in precipitation indices for the overall land areas with data were only found for the Simple Daily Intensity index, and not for other considered indices such as Heavy Rainfall Days (Alexander et al., 2006).

Recent studies have updated the assessment of the AR4, with more regional results now available (Table 3-2). Overall, this additional evidence confirms that more locations and studies show an increase than a decrease in extreme precipitation, but that there are also wide regional and seasonal variations, and trends in many regions are not statistically significant (Table 3-2).

Recent studies on past and current changes in precipitation extremes in North America, some of which are included in the recent assessment of the CCSP report (Kunkel et al., 2008), have reported an increasing trend over the last half century. Based on station data from Canada, the United States, and Mexico, Peterson et al. (2008a) reported that heavy precipitation has been increasing over 1950-2004, as well as the average amount of precipitation falling on days with precipitation. For the contiguous United States, DeGaetano (2009) showed a 20\% reduction in the return period for extreme precipitation of different return levels over 1950-2007; Gleason et al. (2008) reported an increasing trend in the area experiencing a much above-normal proportion of heavy daily precipitation from 1950 to 2006; and Pryor et al. (2009) provided evidence of increases in the intensity of events above the 95th percentile during the 20th century, with a larger magnitude of the increase at the end of the century. The largest trends toward increased annual total precipitation, number of rainy days, and intense precipitation (e.g., fraction derived from events in excess of the 90th percentile value) were focused on the Great Plains/northwestern Midwest (Pryor et al., 2009). In the core of the North American monsoon region in northwest Mexico, statistically significant positive trends were found in daily precipitation intensity and seasonal contribution of daily precipitation greater than its 95th percentile in the mountain sites for the period 1961-1998. However, no statistically significant changes were found in coastal stations (Cavazos et al., 2008). Overall, the evidence indicates a likely increase in observed heavy precipitation in many regions in North America, despite statistically non-significant trends and some decreases in some subregions (Table 3-2). This general increase in heavy precipitation accompanies a general increase in total precipitation in most areas of the country.

There is low to medium confidence in trends for Central and South America, where spatially varying trends in extreme rainfall events have been observed (Table 3-2). Positive trends in many areas but negative trends in some regions are evident for Central America and northern South America (Dufek and Ambrizzi, 2008; Marengo et al., 2009b; Re and Ricardo Barros, 2009; Sugahara et al., 2009). For the western coast of South America, a decrease in extreme rainfall in many areas and an increase in a few areas are observed (Haylock et al., 2006b).

There is medium confidence in trends in heavy precipitation in Europe, due to partly inconsistent signals across studies and regions, especially in summer (Table 3-2). Winter extreme precipitation has increased in part of the continent, in particular in central-western Europe and European Russia (Zolina et al., 2009), but the trend in summer precipitation has been weak or not spatially coherent (Moberg et al., 2006; Bartholy and Pongracz, 2007; Maraun et al., 2008; Pavan et al., 2008; Zolina et al., 2008; Costa and Soares, 2009; Kyselý, 2009; Durão et al., 2010; Rodda et al., 2010). Increasing trends in 90th, 95th, and 98th percentiles of daily winter precipitation over 1901-2000 were found (Moberg et al., 2006), which has been confirmed by more detailed country-based studies for the United Kingdom (Maraun et al., 2008), Germany (Zolina et al., 2008), and central and eastern Europe (Bartholy and Pongracz, 2007; Kyselý, 2009), while decreasing trends have been found in some regions such as northern Italy (Pavan et al., 2008), Poland (Lupikasza, 2010), and some Mediterranean coastal sites (Toreti et al., 2010). Uncertainties are overall larger in southern Europe and the Mediterranean region, where there is low confidence in the trends (Table 3-2). A recent study (Zolina et al., 2010) has indicated that there has been an increase of about 15 to $20 \%$ in the persistence of wet spells over most of Europe over the last 60 years, which was not associated with an increase of the total number of wet days.

There is low to medium confidence in trends in heavy precipitation in Asia, both at the continental and regional scale for most regions (Table 3-2; see also Alexander et al., 2006). A weak increase in the frequency of extreme precipitation events is observed in northern Mongolia (Nandintsetseg et al., 2007). No systematic spatially coherent trends in the frequency and duration of extreme precipitation events have been 
found in Eastern and Southeast Asia (Choi et al., 2009), central and south Asia (Klein Tank et al., 2006), and Western Asia (X. Zhang et al., 2005; Rahimzadeh et al., 2009). However, statistically significant positive and negative trends were observed at subregional scales within these regions. Heavy precipitation increased in Japan during 1901-2004 (Fujibe et al., 2006), and in India (Rajeevan et al., 2008; Krishnamurthy et al., 2009) especially during the monsoon seasons (Sen Roy, 2009; Pattanaik and Rajeevan, 2010). Both statistically significant increases and decreases in extreme precipitation have been found in China over the period 1951-2000 (Zhai et al., 2005) and 1978-2002 (Yao et al., 2008). In Peninsular Malaysia during 1971-2005 the intensity of extreme precipitation increased and the frequency decreased, while the trend in the proportion of extreme rainfall over total precipitation was not statistically significant (Zin et al., 2009). Heavy precipitation increased over the southern and northern Tibetan Plateau but decreased in the central Tibetan Plateau during 1961-2005 (You et al., 2008).

In southern Australia, there has been a likely decrease in heavy precipitation in many areas, especially where mean precipitation has decreased (Table 3-2). There were statistically significant increases in the proportion of annual/seasonal rainfall stemming from heavy rain days from 1911-2008 and 1957-2008 in northwest Australia (Gallant and Karoly, 2010). Extreme summer rainfall over the northwest of the Swan-Avon River basin in western Australia increased over 1950-2003 while extreme winter rainfall over the southwest of the basin decreased (Aryal et al., 2009). In New Zealand, the trends are positive in the western North and South Islands and negative in the east of the country (Mullan et al., 2008).

There is low to medium confidence in regional trends in heavy precipitation in Africa due to partial lack of literature and data, and due to lack of consistency in reported patterns in some regions (Table 3-2). The AR4 (Trenberth et al., 2007) reported an increase in heavy precipitation over southern Africa, but this appears to depend on the region and precipitation index examined (Kruger, 2006; New et al., 2006; Seleshi and Camberlin, 2006; Aguilar et al., 2009). Central Africa exhibited a decrease in heavy precipitation over the last half century (Aguilar et al., 2009); however, data coverage for large parts of the region was poor. Precipitation from heavy events has decreased in western central Africa, but with low spatial coherence (Aguilar et al., 2009). Rainfall intensity averaged over southern and west Africa has increased (New et al., 2006). There is a lack of literature on changes in heavy precipitation in East Africa (Table 3-2). Camberlin et al. (2009) analyzed changes in components of rainy seasons' variability over the time period 1958-1987 in this region, but did not specifically address trends in heavy precipitation. There were decreasing trends in heavy precipitation over parts of Ethiopia during the period 1965-2002 (Seleshi and Camberlin, 2006).

Changes in hail occurrence are generally difficult to quantify because hail occurrence is not well captured by monitoring systems and because of historical data inhomogeneities. Sometimes, changes in environmental conditions conducive to hail occurrence are used to infer changes in hail occurrence. However, the atmospheric conditions are typically estimated from reanalyses or from radiosonde data and the estimates are associated with high uncertainty. As a result, assessment of changes in hail frequency is difficult. For severe thunderstorms in the region east of the Rocky Mountains in the United States, Brooks and Dotzek (2008) found strong variability but no clear trend in the past 50 years. Cao (2008) identified a robust upward trend in hail frequency over Ontario, Canada. Kunz et al. (2009) found that both hail damage days and convective instability increased during 1974-2003 in a state in southwest Germany. Xie et al. (2008) identified no trend in the mean annual hail days in China from 1960 to the early 1980 s but a statistically significant decreasing trend afterwards.

\section{Causes of Observed Changes}

The observed changes in heavy precipitation appear to be consistent with the expected response to anthropogenic forcing (increase due to enhanced moisture content in the atmosphere; see, e.g., Section 3.2.2.1) but a direct cause-and-effect relationship between changes in external forcing and extreme precipitation had not been established at the time of the AR4. As a result, the AR4 only concluded that it was more likely than not that anthropogenic influence had contributed to a global trend towards increases in the frequency of heavy precipitation events over the second half of the 20th century (Hegerl et al., 2007).

New research since the AR4 provides more evidence of anthropogenic influence on various aspects of the global hydrological cycle (Stott et al., 2010; see also Section 3.2.2), which is directly relevant to extreme precipitation changes. In particular, an anthropogenic influence on atmospheric moisture content is detectable (Santer et al., 2007; Willett et al., 2007; see also Section 3.2.2). Wang and Zhang (2008) show that winter season maximum daily precipitation in North America appears to be statistically significantly influenced by atmospheric moisture content, with an increase in moisture corresponding to an increase in maximum daily precipitation. This behavior has also been seen in model projections of extreme winter precipitation under global warming (Gutowski et al., 2008b). Climate model projections suggest that the thermodynamic constraint based on the Clausius-Clapeyron relation is a good predictor for extreme precipitation changes in a warmer world in regions where the nature of the ambient flows change little (Pall et al., 2007). This indicates that the observed increase in extreme precipitation in many regions is consistent with the expected extreme precipitation response to anthropogenic influences. However, the thermodynamic constraint may not be a good predictor in regions with circulation changes, such as mid- to higher latitudes (Meehl et al., 2005) and the tropics (Emori and Brown, 2005), and in arid regions. Additionally, changes in precipitation extremes with temperature also depend on changes in the moistadiabatic temperature lapse rate, in the upward velocity, and in the temperature when precipitation extremes occur ( $0^{\prime}$ Gorman and Schneider, 2009a,b; Sugiyama et al., 2010). This may explain why there have not been increases in precipitation extremes everywhere, although a low signal-to-noise ratio may also play a role. However, even in 

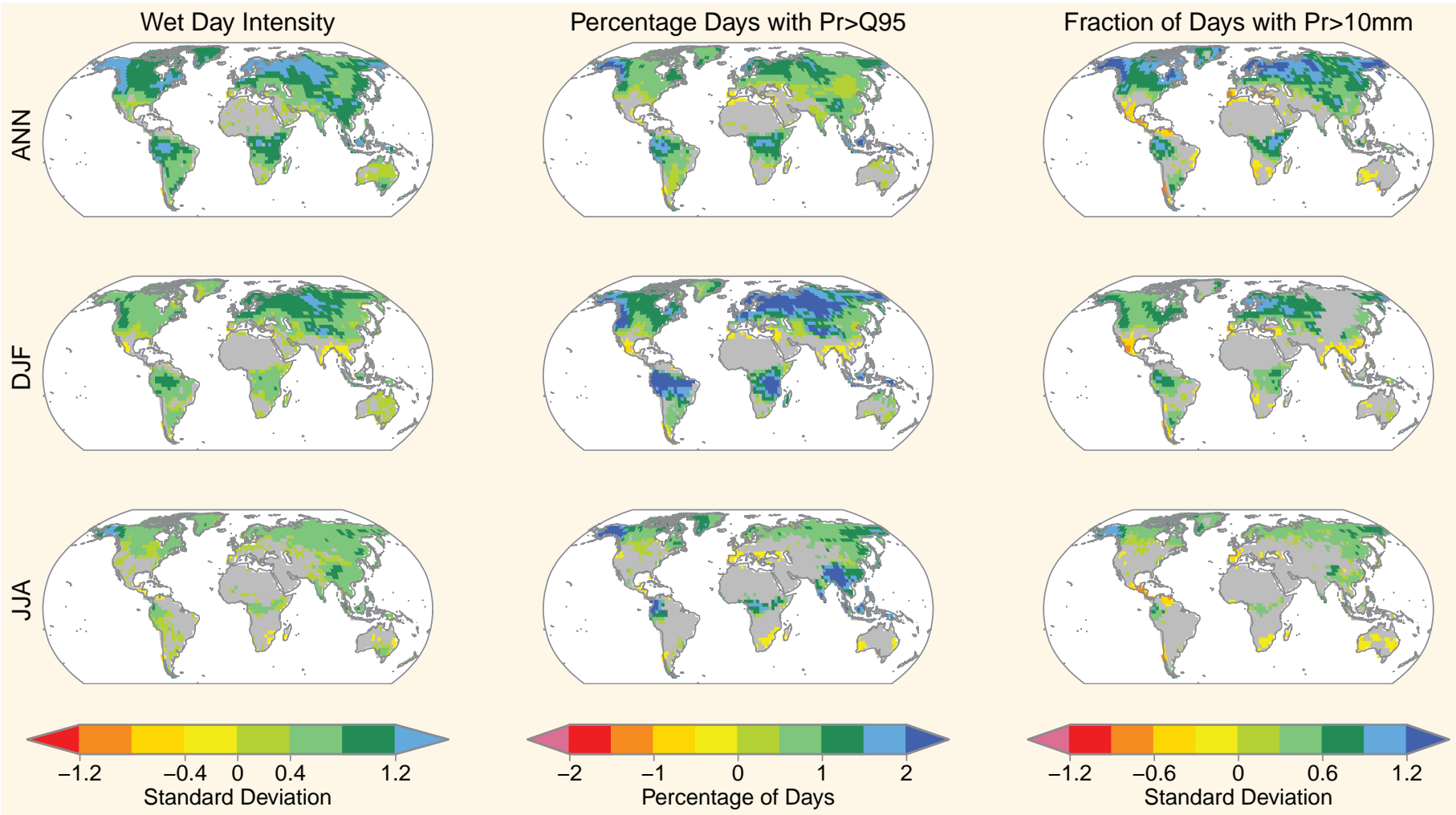

Figure 3-6 | Projected annual and seasonal changes in three indices for daily precipitation (Pr) for 2081-2100 with respect to 1980-1999, based on 17 GCMs contributing to the CMIP3. Left column: wet-day intensity; middle column: percentage of days with precipitation above the $95 \%$ quantile of daily wet day precipitation for that day of the year, calculated from the 1961-1990 reference period; right column: fraction of days with precipitation higher than $10 \mathrm{~mm}$. The changes are computed for the annual time scale (top row) and two seasons (DJF, middle row, and JJA, bottom row) as the fractions/percentages in the 2081-2100 period (based on simulations under emission scenario SRES A2) minus the fractions/percentages of the 1980-1999 period (from corresponding simulations for the 20th century). Changes in wet-day intensity and in the fraction of days with $\mathrm{Pr}>10 \mathrm{~mm}$ are expressed in units of standard deviations, derived from detrended per year annual or seasonal estimates, respectively, from the three 20-year periods 1980-1999, 2046-2065, and 2081-2100 pooled together. Changes in percentages of days with precipitation above the $95 \%$ quantile are given directly as differences in percentage points. Color shading is only applied for areas where at least $66 \%$ (i.e., 12 out of 17) of the GCMs agree on the sign of the change; stippling is applied for regions where at least $90 \%$ (i.e., 16 out of 17 ) of the GCMs agree on the sign of the change. Adapted from Orlowsky and Seneviratne (2011); updating Tebaldi et al. (2006) for additional number of indices and CMIP3 models, and including seasonal time frames. For more details, see Appendix 3.A.

regions where the Clausius-Clapeyron constraint is not closely followed, it still appears to be a better predictor for future changes in extreme precipitation than the change in mean precipitation in climate model projections (Pall et al., 2007). An observational study seems also to support this thermodynamic theory. Analysis of daily precipitation from the Special Sensor Microwave Imager over the tropical oceans shows a direct link between rainfall extremes and temperature: heavy rainfall events increase during warm periods (EI Niño) and decrease during cold periods (Allan and Soden, 2008). However, the observed amplification of rainfall extremes is larger than that predicted by climate models (Allan and Soden, 2008), due possibly to widely varying changes in upward velocities associated with precipitation extremes ( $0^{\prime}$ Gorman and Schneider, 2008). Evidence from measurements in the Netherlands suggests that hourly precipitation extremes may in some cases increase $14 \%$ per degree of warming, which is twice as fast as what would be expected from the Clausius-Clapeyron relationship alone (Lenderink and Van Meijgaard, 2008), though this is still under debate (Haerter and Berg, 2009; Lenderink and van Meijgaard, 2009). A comparison between observed and multi-model simulated extreme precipitation using an optimal detection method suggests that the human-induced increase in greenhouse gases has contributed to the observed intensification of heavy precipitation events over large Northern Hemisphere land areas during the latter half of the 20th century (Min et al., 2011). Pall et al. (2011) linked human influence on global warming patterns with an increased risk of England and Wales flooding in autumn (SeptemberNovember) 2000 that is associated with a displacement in the North Atlantic jet stream. The present assessment based on evidence from new studies and those used in the AR4 is that there is medium confidence that anthropogenic influence has contributed to changes in extreme precipitation at the global scale. However, this conclusion may be dependent on the season and spatial scale. For example, there is now about a 50\% chance that an anthropogenic influence can be detected in UK extreme precipitation in winter, but the likelihood of the detection in other seasons is very small (Fowler and Wilby, 2010).

\section{Projected Changes and Uncertainties}

Regarding projected changes in extreme precipitation, the AR4 concluded that it was very likely that heavy precipitation events, that is, the frequency of heavy precipitation or proportion of total precipitation from heavy precipitation, would increase over most areas of the globe 


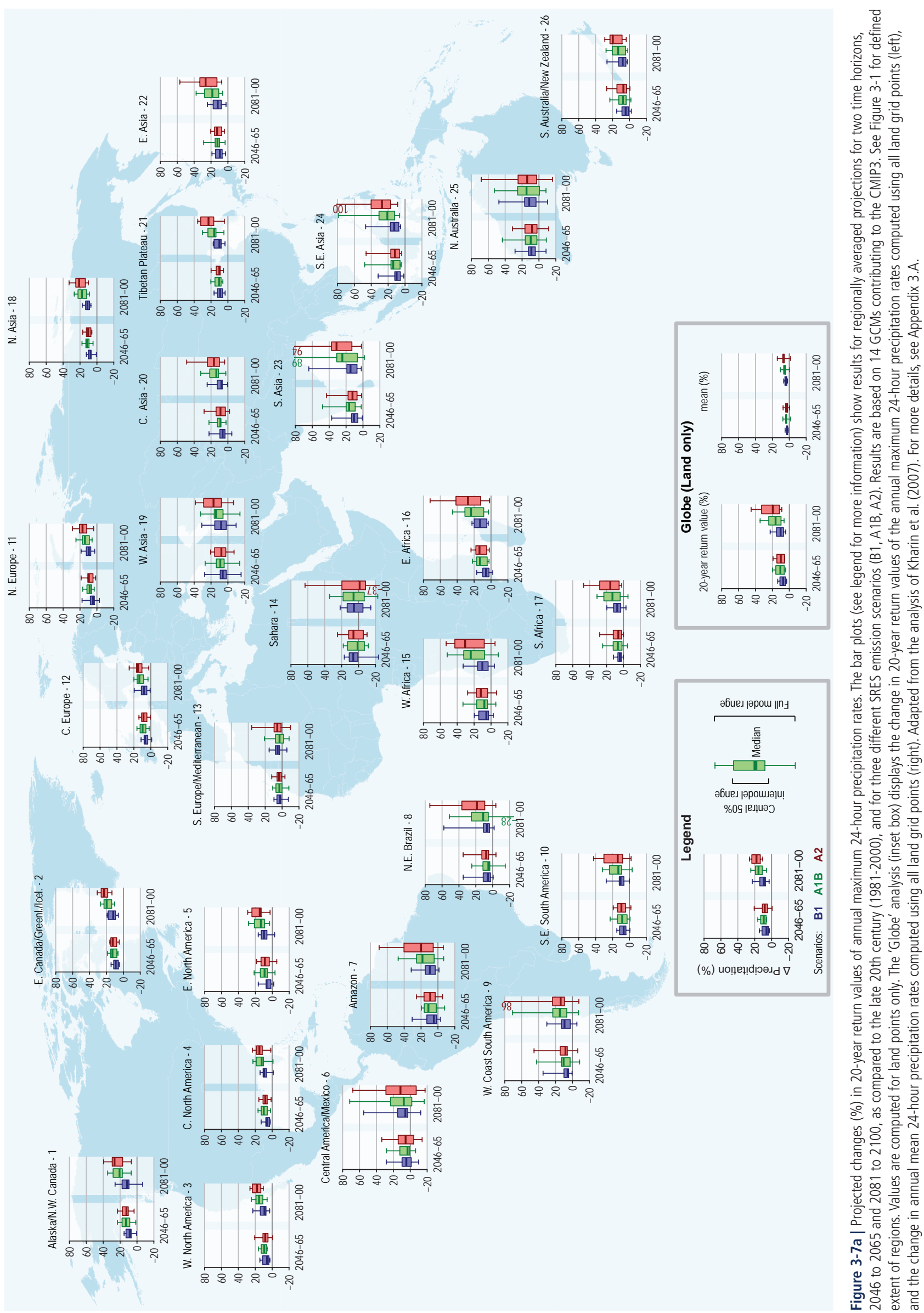




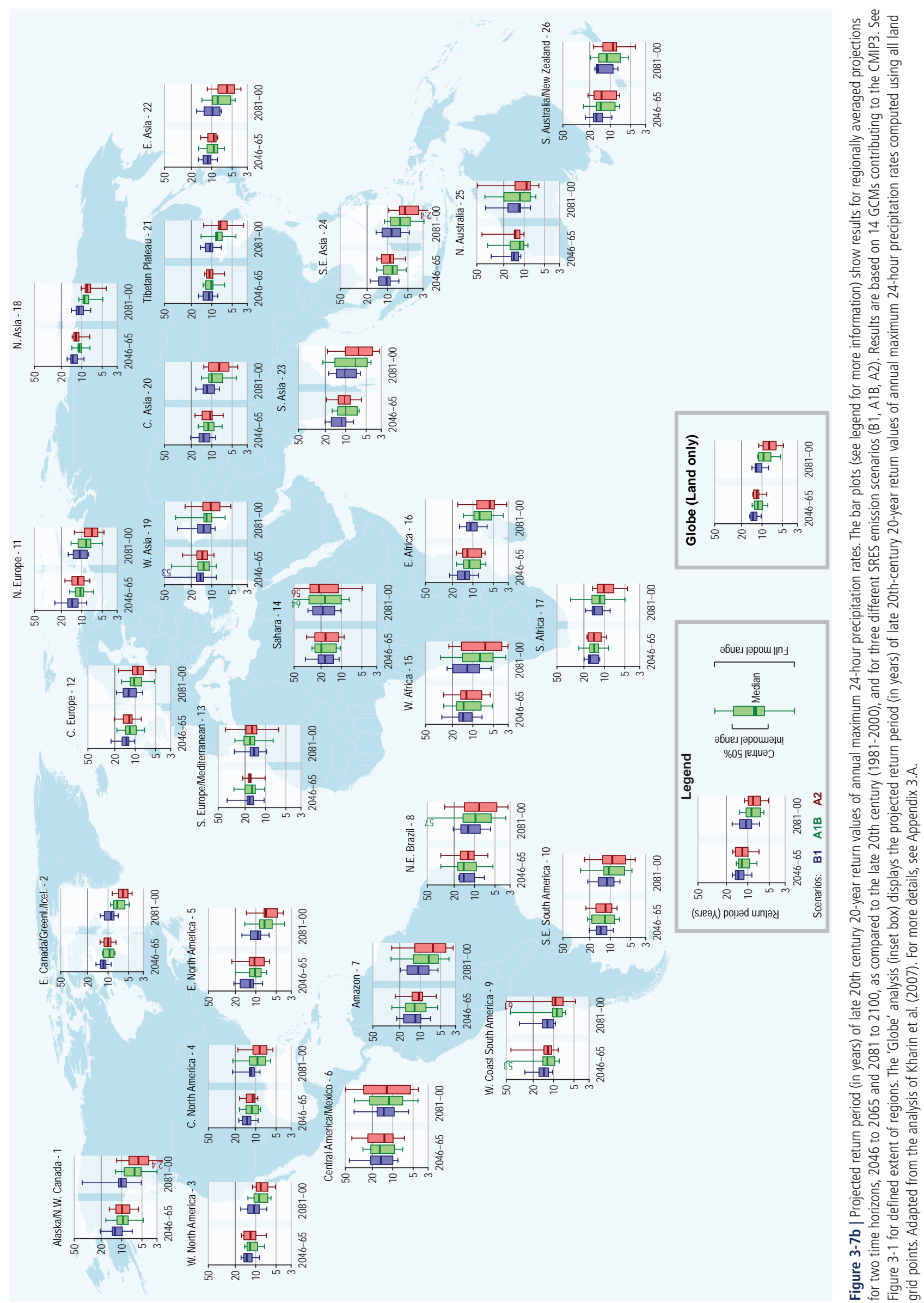


in the 21st century (IPCC, 2007a). The tendency for an increase in heavy daily precipitation events was found in many regions, including some regions in which the total precipitation was projected to decrease.

Post-AR4 analyses of climate model simulations partly confirm this assessment but also highlight fairly large uncertainties and model biases in projections of changes in heavy precipitation in some regions (Section 3.2.3 and Table 3-3). On the other hand, more GCM and RCM ensembles have now been analyzed for some regions (Table 3-3; see also, e.g., Kharin et al., 2007; Kim et al., 2010). At the time of the AR4, Tebaldi et al. (2006) was the main global study available on projected changes in precipitation extremes (e.g., Figure 10.18 of Meehl et al., 2007b). Orlowsky and Seneviratne (2011) extended this analysis to a larger number of GCMs from the CMIP3 ensemble and for seasonal in addition to annual time frames (see also Section 3.3.1). Figure 3-6 provides corresponding analyses of projected annual and seasonal changes of the wet-day intensity, the fraction of days with precipitation above the $95 \%$ quantile of daily wet-day precipitation, and the fraction of days with precipitation above $10 \mathrm{~mm}^{\text {day }}{ }^{-1}$. It should be noted that the $10 \mathrm{~mm}$ day $^{-1}$ threshold cannot be considered extreme in several regions, but highlights differences in projections for absolute and relative thresholds (see also discussion in Box 3-1 and beginning of this section). Figure 3-6 indicates that regions with model agreement (at least 66\%) with respect to changes in heavy precipitation are mostly found in the high latitudes and in the tropics, and in some mid-latitude regions of the Northern Hemisphere in the boreal winter. Regions with at least $90 \%$ model agreement are even more limited and confined to the high latitudes. Overall, model agreement in projected changes is found to be stronger in boreal winter (DJF) than summer (JJA) for most regions. Kharin et al. (2007) analyzed changes in annual maxima of 24-hour precipitation in the outputs of 14 CMIP3 models. Figure 3-7a displays the projected percentage change in the annual maximum of the 24-hour precipitation rate from the late 20th-century 20-year return values, while Figure 3-7b displays the corresponding projected return periods for late 20th-century 20-year return values of the annual maximum 24-hour precipitation rates in the mid-21st century (left) and in late 21st century (right) under three different emission scenarios (SRES B1, A1B, and $A 2$ ). Between the late 20th and the late 21st century, the projected responses of extreme precipitation to future emissions show increased precipitation rates in most regions, and decreases in return periods in most regions in the high latitudes and the tropics and in some regions in the mid-latitudes consistent with projected changes in several indices related to heavy precipitation (see Figure 3-6 and Tebaldi et al., 2006), although there are increases in return periods or only small changes projected in several regions. Except for these regions, the return period for an event of annual maximum 24-hour precipitation with a 20-year return period in the late 20th century is projected to be about 5 to 15 years by the end of the 21 st century. The greatest projected reductions in return period are in high latitudes and some tropical regions. The stronger $\mathrm{CO}_{2}$ emissions scenarios ( $\mathrm{A} 1 \mathrm{~B}$ and $\mathrm{A} 2$ ) lead to greater projected decreases in return period. In some regions with projected decreases in total precipitation (Christensen et al., 2007) such as southern Africa, west Asia, and the west coast of South America, heavy precipitation is nevertheless projected to increase (Figure 3-7, Table 3-3). In some other areas with projected decreases in total precipitation (e.g., Central America and northern South America), however, heavy precipitation is projected to decrease or not change. It should be noted that Figure 3-7 addresses very extreme heavy precipitation events (those expected to occur about once in 20 years) whereas Figure 3-6 addresses less extreme, but still heavy, precipitation events. Projections of changes for these differently defined extreme events may differ.

Future precipitation projected by the CMIP3 models has also been analyzed in a number of studies for various regions using different combinations of the models (see next paragraphs and Table 3-3). In general these studies confirm the findings of global-scale studies by Tebaldi et al. (2006) and Kharin et al. (2007).

By analyzing simulations with a single GCM, Khon et al. (2007) reported a projected general increase in extreme precipitation for the different regions in northern Eurasia especially for winter. Su et al. (2009) found that for the Yangtze River Basin region in 2001-2050, the 50-year heavy precipitation events become more frequent, with return periods falling to below 25 years (relative to 1951-2000 behavior). For the Indian region, the Hadley Centre coupled model HadCM3 projects increases in the magnitude of the heaviest rainfall with a doubling of atmospheric $\mathrm{CO}_{2}$ concentration (Turner and Slingo, 2009). Simulations by 12 GCMs projected an increase in heavy precipitation intensity and mean precipitation rates in east Africa, more severe precipitation deficits in the southwest of southern Africa, and enhanced precipitation further north in Zambia, Malawi, and northern Mozambique (Shongwe et al., 2009, 2011). Rocha et al. (2008) evaluated differences in the precipitation regime over southeastern Africa simulated by two GCMs under present (1961-1990) and future (2071-2100) conditions as a result of anthropogenic greenhouse gas forcing. They found that the intensity of all episode categories of precipitation events is projected to increase practically over the whole region, whereas the number of episodes is projected to decrease in most of the region and for most episode categories. Extreme precipitation is projected to increase over Australia in 2080-2099 relative to 1980-1999 in an analysis of the CMIP3 ensemble, although there are inconsistencies between projections from different models (Alexander and Arblaster, 2009).

High spatial resolution is important for studies of extreme precipitation because the physical processes responsible for extreme precipitation require high spatial resolution to resolve them (e.g., Kim et al., 2010). Post-AR4 studies have employed three approaches to obtain high spatial resolution to project precipitation extremes: high-resolution GCMs, dynamical downscaling using RCMs, and statistical downscaling (see also Section 3.2.3.1). Based on the Meteorological Research Institute and Japan Meteorological Agency 20-km horizontal grid GCM, heavy precipitation was projected to increase substantially in south Asia, the Amazon, and west Africa, with increased dry spell persistence projected in South Africa, southern Australia, and the Amazon at the end of the 21st century (Kamiguchi et al., 2006). In the Asian monsoon region, heavy precipitation was projected to increase, notably in Bangladesh 
and in the Yangtze River basin due to the intensified convergence of water vapor flux in summer. Using statistical downscaling, Wang and Zhang (2008) investigated possible changes in North American extreme precipitation probability during winter from 1949-1999 to 2050-2099. Downscaled results suggested a strong increase in extreme precipitation over the south and central United States but decreases over the Canadian prairies. Projected European precipitation extremes in highresolution studies tend to increase in northern Europe (Frei et al., 2006; Beniston et al., 2007; Schmidli et al., 2007), especially during winter (Haugen and Iversen, 2008; May, 2008), as also highlighted in Table 3-3. Fowler and Ekström (2009) project increases in both short-duration (1-day) and longer-duration (10-day) precipitation extremes across the United Kingdom during winter, spring, and autumn. In summer, model projections for the United Kingdom span the zero change line, although there is low confidence due to poor model performance in this season. Using daily statistics from various models, Boberg et al. $(2009 a, b)$ projected a clear increase in the contribution to total precipitation from more intense events together with a decrease in the number of days with light precipitation. This pattern of change was found to be robust for all European subregions. In double-nested model simulations with a horizontal grid spacing of $10 \mathrm{~km}$, Tomassini and Jacob (2009) projected positive trends in extreme quantiles of heavy precipitation over Germany, although they are relatively small except for the high- $\mathrm{CO}_{2} \mathrm{~A} 2$ emission scenario. For the Upper Mississippi River Basin region during October through March, the intensity of extreme precipitation is projected to increase (Gutowski et al., 2008b). Simulations with a single RCM project an increase in the intensity of extreme precipitation events over most of southeastern South America and western Amazonia in 2071-2100, whereas in northeast Brazil and eastern Amazonia smaller or no changes are projected (Marengo et al., 2009a). Outputs from another RCM indicate an increase in the magnitude of future extreme rainfall events in the Westernport region of Australia, consistent with results based on the CMIP3 ensemble (Alexander and Arblaster, 2009), and the size of this increase is greater in 2070 than in 2030 (Abbs and Rafter, 2008). When both future land use changes and increasing greenhouse gas concentrations are considered in the simulations, tropical and northern Africa are projected to experience less extreme rainfall events by 2025 during most seasons except for autumn (Paeth and Thamm, 2007). Simulations with high-resolution RCMs projected that the frequency of extreme precipitation increases in the warm climate for June through to September in Japan (Nakamura et al., 2008; Wakazuki et al., 2008; Kitoh et al., 2009). An increase in 90th-percentile values of daily precipitation on the Pacific side of the Japanese islands during July in the future climate was projected with a $5-\mathrm{km}$ mesh cloud-systemresolving non-hydrostatic RCM (Kanada et al., 2010b).

Post-AR4 studies indicate that the projection of precipitation extremes is associated with large uncertainties, contributed by the uncertainties related to GCMs, RCMs, and statistical downscaling methods, and by natural variability of the climate. Kyselý and Beranova (2009) examined scenarios of change in extreme precipitation events in 24 future climate runs of 10 RCMs driven by two GCMs, focusing on a specific area of central Europe with complex orography. They demonstrated that the inter- and intra-model variability and related uncertainties in the pattern and magnitude of the change are large, although they also show that the projected trends tend to agree with those recently observed in the area, which may strengthen their credibility. May (2008) reported an unrealistically large projected precipitation change over the Baltic Sea in summer in an RCM, apparently related to an unrealistic projection of Baltic Sea warming in the driving GCM. Frei et al. (2006) found large model differences in summer when RCM formulation contributes significantly to scenario uncertainty. In exploring the ability of two statistical downscaling models to reproduce the direction of the projected changes in indices of precipitation extremes, Hundecha and Bardossy (2008) concluded that the statistical downscaling models seem to be more reliable during seasons when local climate is determined by largescale circulation than by local convective processes. Themeßl et al. (2011) merged linear and nonlinear empirical-statistical downscaling techniques with bias correction methods, and demonstrated their ability to drastically reduce RCM error characteristics. The extent to which the natural variability of the climate affects our ability to project the anthropogenically forced component of changes in daily precipitation extremes was investigated by Kendon et al. (2008). They show that annual to multidecadal natural variability across Europe may contribute to substantial uncertainty. Also, Kiktev et al. (2009) performed an objective comparison of climatologies and historical trends of temperature and precipitation extremes using observations and 20th-century climate simulations. They did not detect significant similarity between simulated and actual patterns of the indices of precipitation extremes in most cases. Moreover, Allan and Soden (2008) used satellite observations and model simulations to examine the response of tropical precipitation events to naturally driven changes in surface temperature and atmospheric moisture content. The observed amplification of rainfall extremes was larger than that predicted by models. The underestimation of rainfall extremes by the models may be related to the coarse spatial resolution used in the model simulations - the magnitude of changes in precipitation extremes depends on spatial resolution (Kitoh et al., 2009) - suggesting that projections of future changes in rainfall extremes in response to anthropogenic global warming may be underestimated.

Confidence is still low for hail projections particularly due to a lack of hail-specific modelling studies, and a lack of agreement among the few available studies. There is little information in the AR4 regarding projected changes in hail events, and there has been little new literature since the AR4. Leslie et al. (2008) used coupled climate model simulations under the SRES A1B scenario to estimate future changes in hailstorms in the Sydney Basin, Australia. Their future climate simulations show an increase in the frequency and intensity of hailstorms out to 2050, and they suggest that the increase will emerge from the natural background variability within just a few decades. This result offers a different conclusion from the modelling study of Niall and Walsh (2005), which simulated Convective Available Potential Energy (CAPE) for southeastern Australia in an environment containing double the pre-industrial concentrations of equivalent $\mathrm{CO}_{2}$. They found a statistically significant projected decrease in CAPE values and concluded that "it is possible that there will be a decrease in the frequency of hail in southeastern 
Australia if current rates of $\mathrm{CO}_{2}$ emission are sustained," assuming the strong relationship between hail incidence and the CAPE for 1980-2001 remains unchanged under enhanced greenhouse conditions.

In summary, it is likely that there have been statistically significant increases in the number of heavy precipitation events (e.g., 95th percentile) in more regions than there have been statistically significant decreases, but there are strong regional and subregional variations in the trends (i.e., both between and within regions considered in this report; Figure 3-1 and Tables 3-2 and 3-3). In particular, many regions present statistically non-significant or negative trends, and, where seasonal changes have been assessed, there are also variations between seasons (e.g., more consistent trends in winter than in summer in Europe). The overall most consistent trends toward heavier precipitation events are found in North America (likely increase over the continent). There is low confidence in observed trends in phenomena such as hail because of historical data inhomogeneities and inadequacies in monitoring systems. Based on evidence from new studies and those used in the AR4, there is medium confidence that anthropogenic influence has contributed to intensification of extreme precipitation at the global scale. There is almost no literature on the attribution of changes in hail extremes, thus no assessment can be provided for these at this point in time. Projected changes from both global and regional studies indicate that it is likely that the frequency of heavy precipitation or proportion of total rainfall from heavy falls will increase in the 21st century over many areas on the globe, especially in the high latitudes and tropical regions, and northern mid-latitudes in winter. Heavy precipitation is projected to increase in some (but not all) regions with projected decreases of total precipitation (medium confidence). For a range of emission scenarios (A2, A1B, and B1), projections indicate that it is likely that a 1-in-20 year annual maximum 24-hour precipitation rate will become a 1 -in- 5 to -15 year event by the end of 21 st century in many regions. Nevertheless, increases or statistically non-significant changes in return periods are projected in some regions.

\subsubsection{Wind}

Extreme wind speeds pose a threat to human safety, maritime and aviation activities, and the integrity of infrastructure. As well as extreme wind speeds, other attributes of wind can cause extreme impacts. Trends in average wind speed can influence potential evaporation and in turn water availability and droughts (e.g., McVicar et al., 2008; see also Section 3.5.1 and Box 3-3). Sustained mid-latitude winds can elevate coastal sea levels (e.g., McInnes et al., 2009b), while longer-term changes in prevailing wind direction can cause changes in wave climate and coastline stability (Pirazzoli and Tomasin, 2003; see also Sections 3.5.4 and 3.5.5). Aeolian processes exert significant influence on the formation and evolution of arid and semi-arid environments, being strongly linked to soil and vegetation change (0kin et al., 2006). A rapid shift in wind direction may reposition the leading edge of a forest fire (see Section 4.2.2.2; Mills, 2005) while the fire itself may generate a local circulation response such as tornado genesis (e.g., Cunningham and Reeder, 2009). Unlike other weather and climate elements such as temperature and rainfall, extreme winds are often considered in the context of the extreme phenomena with which they are associated such as tropical and extratropical cyclones (see also Sections 3.4.4 and 3.4.5), thunderstorm downbursts, and tornadoes. Although wind is often not used to define the extreme event itself (Peterson et al., 2008b), wind speed thresholds may be used to characterize the severity of the phenomenon (e.g., the Saffir-Simpson scale for tropical cyclones). Changes in wind extremes may arise from changes in the intensity or location of their associated phenomena (e.g., a change in local convective activity) or from other changes in the climate system such as the movement of large-scale circulation patterns. Wind extremes may be defined by a range of quantities such as high percentiles, maxima over a particular time scale (e.g., daily to yearly), or storm-related highest values. Wind gusts, which are a measure of the highest winds in a short time interval (typically 3 seconds), may be evaluated in models using gust parameterizations that are applied to the maximum daily nearsurface wind speed (e.g., Rockel and Woth, 2007).

Over paleoclimatic time scales, proxy data have been used to infer circulation changes across the globe from the mid-Holocene ( 6000 years ago) to the beginning of the industrial revolution (Wanner et al., 2008). Over this period, there is evidence for changes in circulation patterns across the globe. The Inter-Tropical Convergence Zone (ITCZ) moved southward, leading to weaker monsoons across Asia (Haug et al., 2001). The Walker circulation strengthened and Southern Ocean westerlies moved northward and strengthened, affecting southern Australia, New Zealand, and southern South America (Shulmeister et al., 2006; Wanner et al., 2008), and an increase in ENSO variability and frequency occurred (Rein et al., 2005; Wanner et al., 2008). There is also weaker evidence for a change toward a lower Northern Atlantic Oscillation (NAO), implying weaker westerly winds over the north Atlantic (Wanner et al., 2008). While the changes in the Northern Hemisphere were attributed to changes in orbital forcing, those in the Southern Hemisphere were more complex, possibly reflecting the additional role on circulation of heat transport in the ocean. Solar variability and volcanic eruptions may also have contributed to decadal to multi-centennial fluctuations over this time period (Wanner et al., 2008).

The AR4 did not specifically address changes in extreme wind although it did report on wind changes in the context of other phenomena such as tropical and extratropical cyclones and oceanic waves and concluded that mid-latitude westerlies had increased in strength in both hemispheres (Trenberth et al., 2007). Direct investigation of changes in wind climatology has been hampered by the sparseness of long-term, highquality wind measurements from terrestrial anemometers arising from the influence of changes in instrumentation, station location, and surrounding land use (e.g., Cherry, 1988; Pryor et al., 2007; Jakob, 2010; see also Section 3.2.1). Nevertheless, a number of recent studies report trends in mean and extreme wind speeds in different parts of the world based on wind observations and reanalyses. 
Over North America, a declining trend in 50th and 90th percentile wind speeds has been reported for much of the United States over 1973 to 2005 (Pryor et al., 2007) and in 10-m hourly wind data over 1953-2006 over western and most of southern Canada (Wan et al., 2010). An increasing trend has been reported in average winds over Alaska over 1955-2001 by Lynch et al. (2004) and over the central Canadian Arctic in all seasons and in the Maritimes in spring and autumn by Wan et al. (2010) as well as in annual maximum winds in a regional reanalysis over the southern Maritimes from 1979-2003 (Hundecha et al., 2008). Over China, negative trends have been reported in 10-m monthly mean and 95th percentile winds over 1969-2005 (Guo et al., 2011), in daily maximum wind speeds over 1956-2004 by Jiang et al. (2010a), and in 2-m average winds over the Tibetan plateau from 1966-2003 (Y. Zhang et al., 2007), confirming earlier declining trends in mean and strong 10-m winds reported by Xu et al. (2006). Over Europe, Smits et al. (2005) found declining trends in extreme winds (those occurring on average 10 and 2 times per year) in 10-m anemometer data over 1962-2002. Pirazolli and Tomasin (2003) reported a generally declining trend in both annual mean and annual maximum winds from 1951 to the mid1970 s and an increasing trend since then, from observations in the central Mediterranean region. Similar to the mostly declining trends found in Northern Hemisphere studies of surface wind observations, Vautard et al. (2010) also found mostly declining trends in surface wind observations across the continental northern mid-latitudes and a stronger decline in extreme winds compared to mean winds in surface wind measurements. In the Southern Hemisphere, McVicar et al. (2008) reported declines in 2-m mean wind speed over $88 \%$ of Australia (significant over $57 \%$ of the country) over 1975-2006 and positive trends over about $12 \%$ of the mainland interior and southern and eastern coastal regions including Tasmania. In Antarctica, increasing trends in mean wind speeds have been reported over the second half of the 20th century (Turner et al., 2005). With the exception of the robust declines in wind reported over China, studies in most areas are too few in number to draw robust conclusions on wind speed change and even fewer studies have addressed extreme wind change. Some studies report opposite trends between anemometer winds and reanalysis data sets in some areas (Smits et al., 2005; McVicar et al., 2008; Vautard et al., 2010); however, comparisons of surface anemometer data at $10 \mathrm{~m}$ or lower with reanalysis-derived $10-\mathrm{m}$ data that do not resolve complex surface features is problematic.

Trends in extreme winds have also been inferred from trends in particular phenomena. With regards to tropical cyclones (Section 3.4.4.), no statistically significant trends have been detected in the overall global annual number although a trend has been reported in the intensity of the strongest storms since 1980 [but there is low confidence that any observed long-term (i.e., 40 years or more) increases in tropical cyclone activity are robust, after accounting for past changes in observing capabilities; see Section 3.4.4]. In the mid-latitudes, studies have used proxies for wind such as pressure tendencies or geostrophic winds calculated from triangles of pressure (geo-winds) over Europe (e.g., Barring and von Storch, 2004; Matulla et al., 2008; Allan et al., 2009; Barring and Fortuniak, 2009; X.L. Wang et al., 2009b) and Australia (e.g.,
Alexander and Power, 2009; Alexander et al., 2011). For Europe, these studies suggest that storm activity was higher around 1900 and in the 1990s and lower in the 1960s and 1970s, although X.L. Wang et al. (2009b) note that seasonal trends behave differently than annual trends. In general, long-term trends differ between the different available studies as well as studies that focus on the period for which reanalysis data exist (e.g., Raible, 2007; Leckebusch et al., 2008; Della-Marta et al., 2009; Nissen et al., 2010), and strong inter-decadal variability is also often reported (e.g., Allan et al., 2009; X.L. Wang et al., 2009b; Nissen et al., 2010). Over southeast Australia, a decline in storm activity since around 1885 has been reported (Alexander and Power, 2009; Alexander et al., 2011). See Section 3.4.5 for more discussion of extratropical cyclones. Regarding other phenomena associated with extreme winds, such as thunderstorms, tornadoes, and mesoscale convective complexes, studies are too few in number to assess the effect of their changes on extreme winds. As well, historical data inhomogeneities mean that there is low confidence in any observed trends in these small-scale phenomena.

The AR4 reported for the mid-latitudes that trends in the Northern and Southern Annular Modes, which correspond to sea level pressure reductions over the poles, are likely related in part to human activity, and this in turn has affected storm tracks and wind patterns in both hemispheres (Hegerl et al., 2007). The relationship between mean and severe winds and natural modes of variability has been investigated in several postAR4 studies. On the Canadian west coast, Abeysirigunawardena et al. (2009) found that higher extreme winds tend to occur during the negative (i.e., cold) ENSO phase. The generally increasing trend in mean wind speeds over recent decades in Antarctica is consistent with the change in the nature of the Southern Annular Mode toward its high index state (Turner et al., 2005). Donat et al. (2010b) concluded that $80 \%$ of storm days in central Europe are connected with westerly flows that occur primarily during the positive phase of the NAO. Declining trends in wind over China have mainly been linked to circulation changes due to a weaker land-sea thermal contrast (Xu et al., 2006; Jiang et al., 2010a; Guo et al., 2011). Vautard et al. (2010) attribute the slowdown in mid to high percentiles of surface winds over most of the continental northern mid-latitudes to changes in atmospheric circulation (10-50\%) and an increase in surface roughness due to biomass increases (25-60\%), which are supported by RCM simulations. X.L. Wang et al. (2009a) formally detected a link between external forcing and positive trends in the high northern latitudes and negative trends in the northern midlatitudes using a proxy for wind (geostrophic wind energy) in the boreal winter. Trends in mean and annual maximum winds in the central Mediterranean region were found to be positively correlated with temperature but not with the NAO index (Pirazzoli and Tomasin, 2003). Nissen et al. (2010) used cyclone tracking to identify associated strong winds in reanalysis data from 1957 to 2002 and found a positive trend in the central Mediterranean region and southern Europe and a negative trend over the western Mediterranean region.

Projections of wind speed changes and particularly wind extremes were not specifically addressed in the AR4 although references to wind speed were made in relation to other variables and phenomena such as 

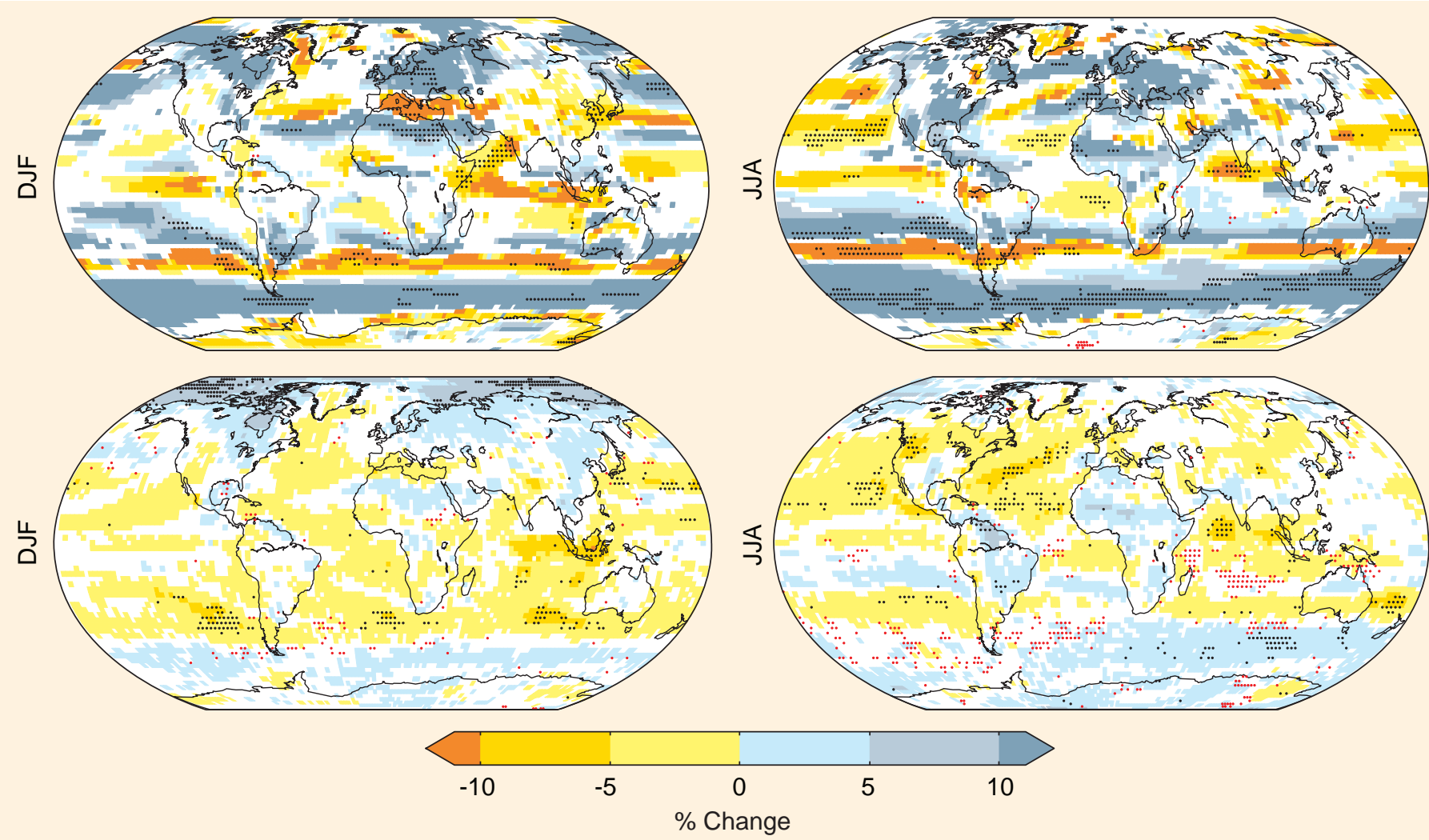

Figure 3-8 | Averaged changes from a 19-member ensemble of CMIP3 GCMs in the mean of the daily averaged 10-m wind speeds (top) and 99th percentile of the daily averaged 10-m wind speeds (bottom) for the period 2081-2100 relative to 1981-2000 (\% change) for December to February (left) and June to August (right) plotted only where more than $66 \%$ of the models agree on the sign of the change. Black stippling indicates areas where more than $90 \%$ of the models agree on the sign of the change. Red stippling indicates areas where more than $66 \%$ of models agree on a small change between $\pm 2 \%$. Adapted from Mclnnes et al. (2011); for more details see Appendix $3 . A$.

mid-latitude storm tracks, tropical cyclones, and ocean waves (Christensen et al., 2007; Meehl et al., 2007b). Meehl et al. (2007b) projected a likely increase in tropical cyclone extreme winds in the future and provided more evidence for a projected poleward shift of the storm tracks and associated changes in wind patterns. Since the AR4, new studies have focused on future changes in winds. Gastineau and Soden (2009) reported a decrease in 99th-percentile winds at $850 \mathrm{hPa}$ in the tropics and an increase in the extratropics in a 17-member multimodel ensemble over 2081-2100 relative to 1981-2000. McInnes et al. (2011) presented spatial maps of multi-model agreement in mean and 99th-percentile 10-m wind change between 1981-2000 and 2081-2100 in a 19-member ensemble (see Figure 3-8). These show an increase in mean winds over Europe, parts of Central and North America, the tropical South Pacific, and the Southern Ocean. Mean wind speed declines occur along the equator reflecting a slowdown in the Walker circulation (Collins et al., 2010) (and in the vicinity of the subtropical ridge in both hemispheres which, together with the strengthening of winds further poleward, reflect the contraction toward the poles of the mid-latitude storm tracks; see Section 3.4.5). Seasonal differences are also apparent with more extensive mean wind increases in the Arctic and parts of the northern Pacific in DJF and decreases over most of the northern Pacific in JJA. The 99th-percentile wind changes show declines over most ocean areas except the northern Pacific and Arctic and Southern Ocean south of $40^{\circ} \mathrm{S}$ in DJF, the south Pacific between about 10 and $25^{\circ} \mathrm{S}$ in JJA, and the Southern Ocean south of $50^{\circ} \mathrm{S}$ in JJA. Increases in 99th-percentile winds occur over the Arctic and large parts of the continental area in the Northern Hemisphere in DJF and in Africa, northern Australia, and Central and South America in JJA. Despite the projections displayed in Figure $3-8$, the relatively few studies of projected extreme winds, combined with shortcomings in the simulation of extreme winds and the different models, regions, and methods used to develop projections of this quantity, means that we have low confidence in projections of changes in strong winds.

Regional increases in winter wind storm risk over Europe due to changes in storm tracks are also supported by a number of regional studies (e.g., Pinto et al., 2007b; Debernard and Roed, 2008; Leckebusch et al., 2008; Sterl et al., 2009; Donat et al., 2010a,b, 2011). However, GCMs at their current resolution are unable to resolve small-scale phenomena such as tropical cyclones, tornadoes, and mesoscale convective complexes that are associated with particularly severe winds, although as noted by McInnes et al. (2011) these winds would typically be more extreme than 99th percentile. There is evidence to suggest an increase in extreme winds from tropical cyclones in the future (see Section 3.4.4). An increase in atmospheric greenhouse gas concentrations may cause some of the atmospheric conditions conducive to tornadoes such as atmospheric instability to increase due to increasing temperature and humidity, while others such as vertical shear to decrease due to a reduced pole-to-equator temperature gradient (Diffenbaugh et al., 2008), but the literature on these phenomena is extremely limited at 
this time. There is thus low confidence in projections of changes in such small-scale systems because of limited studies, inability of climate models to resolve these phenomena, and possible competing factors affecting future changes. Confidence in the extreme wind changes is therefore lower in the regions most influenced by these phenomena irrespective of whether there is high agreement between GCMs on the sign of the wind speed change.

In addition to studies using GCMs there have also been several recent studies employing RCMs. Those focusing on Europe (e.g., Beniston et al., 2007; Rockel and Woth, 2007; Haugen and Iversen, 2008; Rauthe et al., 2010) also provide a general picture of an increasing trend in extreme winds over northern Europe despite a range of different downscaling models used, the different GCMs in which the downscaling is undertaken, and different metrics used to quantify extreme winds. Small-scale polar lows that typically form north of $60^{\circ} \mathrm{N}$ have been found to decline in frequency in RCM simulations downscaled from a GCM under different emission scenarios and this is related to greater stability over the region due to mid-troposphere temperatures warming faster than sea surface temperatures over the region (Zahn and von Storch, 2010). In other parts of the world there have been very few studies. Over China, Jiang et al. (2010b) projected decreases in annual and winter mean wind speed based on two RCMs that downscale two different GCMs. Over North America, statistical downscaling of winds from four GCMs over five airports in the northwest United States indicated declines in summer wind speeds and less certain changes in winter (Sailor et al., 2008).

A number of recent studies have addressed observed changes in wind speed across different parts of the globe, but due to the various shortcomings associated with anemometer data and the inconsistency in anemometer and reanalysis trends in some regions, we have low confidence in wind trends and their causes at this stage. We also have low confidence in how the observed trends in mean wind speed relate to trends in extreme winds. The few studies of projected extreme winds, combined with shortcomings in the simulation of extreme winds and the different models, regions, and methods used to develop projections of this quantity, mean that we have low confidence in projections of changes in extreme winds (with the exception of changes associated with tropical cyclones; Section 3.4.4). There is low confidence in projections of small-scale phenomena such as tornadoes because competing physical processes may affect future trends and because climate models do not simulate such phenomena.

\subsection{Observed and Projected Changes in Phenomena Related to Weather and Climate Extremes}

\subsubsection{Monsoons}

Changes in monsoon-related extreme precipitation and winds due to climate change are not well understood. Generally, precipitation is the most important variable, but it is also a variable associated with larger uncertainties in climate simulations and projections (Wang et al., 2005; Kang and Shukla, 2006). Changes in monsoons should be better depicted by large-scale dynamics, circulation, or moisture convergence more broadly than via precipitation only. However, few studies have focused on observed changes in the large-scale and regional monsoon circulations. Hence, in this section, we focus mostly on monsoon-induced changes in total and seasonal rainfall, with most discussions of intense rainfall covered in Section 3.3.2.

Modeling experiments to assess paleo-monsoons suggest that in the past, during the Holocene due to orbital forcing on a millennial time scale, there was a progressive southward shift of the Northern Hemisphere summer position of the ITCZ around 8,000 years ago. This was accompanied by a pronounced weakening of the monsoon rainfall systems in Africa and Asia and increasing dryness on both continents, while in South America the monsoon was weaker and drier than in the present, as suggested both by models and paleoclimatic indicators (Wanner et al., 2008).

The delineation of the global monsoon has been mostly performed using rainfall data or outgoing longwave radiation (OLR) fields (Kim et al., 2008). Lau and Wu (2007) identified two opposite time evolutions in the occurrence of rainfall events in the tropics: a negative trend in moderate rain events and a positive trend in heavy and light rain events. Positive trends in intense rain were located in deep convective cores of the ITCZ, South Pacific Convergence Zone, Indian Ocean, and monsoon regions.

In the Indo-Pacific region, covering the southeast Asian and north Australian monsoon, Caesar et al. (2011) found low spatial coherence in trends in precipitation extremes across the region between 1971 and 2003. In the few cases where statistically significant trends in precipitation extremes were identified, there was generally a trend towards wetter conditions, in common with the global results of Alexander et al. (2006). Liu et al. (2011) reported a decline in recorded precipitation events in China over 1960-2000, which was mainly accounted for by a decrease in light precipitation events, with intensities of $0.1-0.3 \mathrm{~mm}^{2}$ day $^{-1}$. Some of the extreme precipitation appeared to be positively correlated with a La Niña-like sea surface temperature (SST) pattern, but without suggesting the presence of a trend. With regard to wind changes, Guo et al. (2011) analyzed near-surface wind speed change in China and its monsoon regions from 1969 to 2005 and showed a statistically significant weakening in annual and seasonal mean wind.

For the Indian monsoon, Rajeevan et al. (2008) showed that extreme rain events have an increasing trend between 1901 and 2005, but the trend is much stronger after 1950. Sen Roy (2009) investigated changes in extreme hourly rainfall in India, and found widespread increases in heavy precipitation events across India, mostly in the high-elevation regions of the northwestern Himalaya as well as along the foothills of the Himalaya extending south into the Indo-Ganges basin, and particularly during the summer monsoon season during 1980-2002. 
In the African monsoon region, Fontaine et al. (2011) investigated recent observed trends using high-resolution gridded precipitation (period 1979-2002), OLR, and reanalyses. Their results revealed a rainfall increase in North Africa since the mid-1990s. Over the longer term, however, Zhou et al. (2008a,b) and Wang and Ding (2006) reported an overall decreasing long-term trend in global land monsoon rainfall during the last 54 years, which was mainly caused by decreasing rainfall in the North African and South Asian monsoons.

For the American monsoon regions, Cavazos et al. (2008) reported increases in the intensity of precipitation in the mountain sites of the northwestern Mexico section of the North American monsoon over the 1961-1998 period, apparently related to an increased contribution from heavy precipitation derived from tropical cyclones. Arriaga-Ramírez and Cavazos (2010) found that total and extreme rainfall in the monsoon region of western Mexico and the US southwest presented a statistically significant increase during 1961-1998, mainly in winter. Groisman and Knight (2008) found that consecutive dry days (see Box 3-3 for definition) have significantly increased in the US southwest. On the other hand, increases in heavy precipitation during 1960-2000 in the South American monsoon have been documented by Marengo et al. $(2009 a, b)$ and Rusticucci et al. (2010). Studies using circulation fields such as $850 \mathrm{hPa}$ winds or moisture flux have been performed for the South American monsoon system for assessments of the onset and end of the monsoon, and indicate that the onset exhibits a marked interannual variability linked to variations in SST anomalies in the eastern Pacific and tropical Atlantic (Gan et al., 2006; da Silva and de Carvalho, 2007; Raia and Cavalcanti, 2008; Nieto-Ferreira and Rickenbach, 2011).

Attributing the causes of changes in monsoons is difficult in part because there are substantial inter-model differences in representing Asian monsoon processes (Christensen et al., 2007). Most models simulate the general migration of seasonal tropical rain, although the observed maximum rainfall during the monsoon season along the west coast of India, the North Bay of Bengal, and adjoining northeast India is poorly simulated by many models due to limited resolution. Bollasina and Nigam (2009) show the presence of large systematic biases in coupled simulations of boreal summer precipitation, evaporation, and SST in the Indian Ocean. Many of the biases are pervasive, being common to most simulations.

The observed negative trend in global land monsoon rainfall is better reproduced by atmospheric models forced by observed historical SST than by coupled models without explicit forcing by observed ocean temperatures (Kim et al., 2008). This trend in the east Asian monsoon is strongly linked to the warming trend over the central eastern Pacific and the western tropical Indian Ocean (Zhou et al., 2008b). For the west African monsoon, Joly and Voldoire (2010) explore the role of Gulf of Guinea SSTs in its interannual variability. In most of the studied CMIP3 simulations, the interannual variability of SST is very weak in the Gulf of Guinea, especially along the Guinean Coast. As a consequence, the influence on the monsoon rainfall over the African continent is poorly reproduced. It is suggested that this may be due to the counteracting effects of the Pacific and Atlantic basins over the last decades. The decreasing long-term trend in north African summer monsoon rainfall may be due to the atmosphere response to observed SST variations (Hoerling et al., 2006; Zhou et al., 2008b; Scaife et al., 2009). A similar trend in global monsoon precipitation in land regions is reproduced in CMIP3 models' 20th-century simulations when they include anthropogenic forcing, and for some simulations natural forcing (including volcanic forcing) as well, though the trend is much weaker in general, with the exception of one model (HadCM3) capable of producing a trend of similar magnitude (Li et al., 2008). The decrease in east Asian monsoon rainfall also seems to be related to tropical SST changes (Li et al., 2008), and the less spatially coherent positive trends in precipitation extremes in the southeast Asian and north Australian monsoons appear to be positively correlated with a La Niña-like SST pattern (Caesar et al., 2011).

A variety of factors, natural and anthropogenic, have been suggested as possible causes of variations in monsoons. Changes in regional monsoons are strongly influenced by the changes in the states of dominant patterns of climate variability such as ENSO, the Pacific Decadal Oscillation (PDO), the Northern Annular Mode (NAM), the Atlantic Multi-decadal Oscillation (AM0), and the Southern Annular Mode (SAM) (see also Sections 3.4.2 and 3.4.3). Additionally, model-based evidence has suggested that land surface processes and land use changes could in some instances significantly impact regional monsoons. Tropical land cover change in Africa and southeast Asia appears to have weaker local climatic impacts than in Amazonia (Voldoire and Royer, 2004; Mabuchi et al., 2005a,b). Grimm et al. (2007) and Collini et al. (2008) explored possible feedbacks between soil moisture and precipitation during the early stages of the monsoon in South America, when the surface is not sufficiently wet, and soil moisture anomalies may thus also modulate the development of precipitation. However, the influence of historical land use on the monsoon is difficult to quantify, due both to the poor documentation of land use and difficulties in simulating the monsoon at fine scales. The impact of aerosols (black carbon and sulfate) on changes in rainfall variability and amounts in monsoon regions has been discussed by Meehl et al. (2008), Lau et al. (2006), and Silva Dias et al. (2002). These studies suggest that there are still large uncertainties and a strong model dependency in the representation of the relevant land surface processes and the role of aerosol direct forcing, and resulting interactions (e.g., in the case of land use forcing; Pitman et al., 2009).

Regarding projections of change in the monsoons, the AR4 (Christensen et al., 2007) concluded: "There is a tendency for monsoonal circulations to result in increased precipitation due to enhanced moisture convergence, despite a tendency towards weakening of the monsoonal flows themselves. However, many aspects of tropical climatic responses remain uncertain." Held and Soden (2006) demonstrate that an increase in the hydrological cycle is accompanied by a global weakening of the largescale circulation. As global warming is projected to lead to faster warming over land than over the oceans (e.g., Meehl et al., 2007b; Sutton et al., 2007), the continental-scale land-sea thermal contrast, a major factor affecting monsoon circulations, will become stronger in summer. Based on this projection, a simple scenario is that the summer 
monsoon will be stronger and the winter monsoon will be weaker in the future than now. However, model results derived from the analyses of 15 CMIP3 global models are not as straightforward as implied by this simple consideration (Tanaka et al., 2005), as they show a weakening of these tropical circulations by the late 21st century compared to the late 20th century. In turn, such changes in circulation may lead to changes in precipitation associated with monsoons. For instance, the monsoonal precipitation in Mexico and Central America is projected to decrease in association with increasing precipitation over the eastern equatorial Pacific through changes in the Walker circulation and local Hadley circulation (e.g., Lu et al., 2007). Furthermore, observations and models suggest that changes in monsoons are related at least in part to changes in observed SSTs, as noted above.

At regional scales, there is little consensus in GCM projections regarding the sign of future change in monsoon characteristics, such as circulation and rainfall. For instance, while some models project an intense drying of the Sahel under a global warming scenario, others project an intensification of the rains, and some project more frequent extreme events (Cook and Vizy, 2006). Increases in precipitation are projected in the Asian monsoon (along with an increase in interannual seasonaveraged precipitation variability), and in the southern part of the west African monsoon, but with some decreases in the Sahel in northern summer. In the Australian monsoon in southern summer, an analysis by Moise and Colman (2009) from the entire ensemble mean of CMIP3 simulations suggested no changes in Australian tropical rainfall during the summer and only slightly enhanced interannual variability.

A study of 19 CMIP3 global models reported a projected increase in mean south Asian summer monsoon precipitation of $8 \%$ and a possible extension of the monsoon period (Kripalani et al., 2007). A study (Ashfaq et al., 2009) from the downscaling of the National Center for Atmospheric Research (NCAR) CCSM3 global model using the RegCM3 regional model suggests a weakening of the large-scale monsoon flow and suppression of the dominant intra-seasonal oscillatory modes with overall weakening of the south Asian summer monsoon by the end of the 21st century, resulting in a decrease in summer precipitation in southeast Asia.

Kitoh and Uchiyama (2006) used 15 models under the A1B scenario to analyze the changes in intensity and duration of precipitation in the Baiu-Changma-Meiyu rain band at the end of the 21st century. They found a delay in early summer rain withdrawal over the region extending from the Taiwan province of China, and across the Ryukyu Islands to the south of Japan, contrasted with an earlier withdrawal over the Yangtze Basin. They attributed this feature to El Niño-like mean state changes over the monsoon trough and subtropical anticyclone over the western Pacific region (Meehl et al., 2007b). A southwestward extension of the subtropical anticyclone over the northwestern Pacific Ocean associated with El Niño-like mean state changes and a dry air intrusion in the midtroposphere from the Asian continent to the northwest of Japan provides favorable conditions for intense precipitation in the Baiu season in Japan (Kanada et al., 2010a). Kitoh et al. (2009) projected changes in precipitation characteristics during the east Asian summer rainy season, using a 5-km mesh cloud-resolving model embedded in a 20-km mesh global atmospheric model with CMIP3 mean SST changes. The frequency of heavy precipitation was projected to increase at the end of the 21st century for hourly as well as daily precipitation. Further, extreme hourly precipitation was projected to increase even in the near future (2030s) when the temperature increase is still modest, even though uncertainties in the projection (and even the simulation) of hourly rainfall are still high.

Climate change scenarios for the 21 st century show a weakening of the North American monsoon through a weakening and poleward expansion of the Hadley cell (Lu et al., 2007). The expansion of the Hadley cell is caused by an increase in the subtropical static stability, which pushes poleward the baroclinic instability zone and hence the outer boundary of the Hadley cell. Simple physical arguments (Held and Soden, 2006) predict a slowdown of the tropical overturning circulation under global warming. A few studies (e.g., Marengo et al., 2009a) have projected over the period 1960-2100 a weak tendency for an increase in dry spells. The projections show an increase in the frequency of rainfall extremes in southeastern South America by the end of the 21st century, possibly due to an intensification of the moisture transport from Amazonia by a more frequent/intense low-level jet east of the Andes in the A2 emissions scenario (Marengo et al., 2009a; Soares and Marengo, 2009).

There are many deficiencies in model representation of the monsoons and the processes affecting them, and this reduces confidence in their ability to project future changes. Some of the uncertainty in global and regional climate change projections in the monsoon regions results from the limits in the model representation of resolved processes (e.g., moisture advection), the parameterizations of sub-grid-scale processes (e.g., clouds, precipitation), and model simulations of feedback mechanisms at the global and regional scale (e.g., changes in land use/cover; see also Section 3.1.4). Kharin et al. (2007) made an intercomparison of precipitation extremes in the tropical region in all AR4 models with observed extremes expressed as 20-year return values. They found very large disagreement in the tropics suggesting that some physical processes associated with extreme precipitation are not well represented by the models due to model resolution and physics. Shukla (2007) noted that current climate models cannot even adequately predict the mean intensity and the seasonal variations of the Asian summer monsoon. This reduces confidence in the projected changes in extreme precipitation over the monsoon regions. Many of the important climatic effects of the Madden-Julian Oscillation (MJO, a natural mode of the climate system operating on time scales of about a month), including its impacts on rainfall variability in the monsoons, are still poorly simulated by contemporary climate models (Christensen et al., 2007).

Current GCMs still have difficulties and display a wide range of skill in simulating the subseasonal variability associated with the Asian summer monsoon (Lin et al., 2008b). Most GCMs simulate westward propagation of the coupled equatorial easterly waves, but relatively poor eastward propagation of the MJO and overly weak variances for both the easterly waves and the MJO. Most GCMs are able to reproduce the basic 
characteristics of the precipitation seasonal cycle associated with the South American Monsoon System (SAMS), but there are large discrepancies in the South Atlantic Convergence Zone represented by the models in both intensity and location, and in its seasonal evolution (Vera et al., 2006). In addition, models exhibit large discrepancies in the direction of the changes associated with the summer (SAMS) precipitation, which makes the projections for that region highly uncertain. Lin et al. (2008a) show that the coupled GCMs have significant problems and display a wide range of skill in simulating the North American monsoon and associated intra-seasonal variability.

Most of the models reproduce the monsoon rain belt, extending from southeast to northwest, and its gradual northward shift in early summer, but overestimate the precipitation over the core monsoon region throughout the seasonal cycle and fail to reproduce the monsoon retreat in the fall. The AR4 assessed that models fail in representing the main features of the west African monsoon although most of them do have a monsoonal climate albeit with some distortion (Christensen et al., 2007). Other major sources of uncertainty in projections of monsoon changes are the responses and feedbacks of the climate system to emissions as represented in climate models. These uncertainties are particularly related to the representation of the conversion of emissions into concentrations of radiatively active species (i.e., via atmospheric chemistry and carbon cycle models) and especially those derived from aerosol products of biomass burning, which can affect the onset of the rainy season (Silva Dias et al., 2002). The subsequent response of the physical climate system complicates the nature of future projections of monsoon precipitation. Moreover, the long-term variations of model skill in simulating monsoons and their variations represent an additional source of uncertainty for the monsoon regions, and indicate that the regional reliability of long climate model runs may depend on the time slice for which the output of the model is analyzed.

The AR4 (Hegerl et al., 2007) concluded that the current understanding of climate change in the monsoon regions remains one of considerable uncertainty with respect to circulation and precipitation. With a few exceptions in some monsoon regions, this has not changed. These conclusions have been based on very few studies, there are many issues with model representation of monsoons and the underlying processes, and there is little consensus in climate models, so there is low confidence in projections of changes in monsoons, even in the sign of the change. However, one common pattern is a likely increase in extreme precipitation in monsoon regions (see Section 3.3.2), though not necessarily induced by changes in monsoon characteristics, and not necessarily occurring in all monsoon regions.

\subsubsection{El Niño-Southern Oscillation}

The El Niño-Southern Oscillation (ENSO) is a natural fluctuation of the global climate system caused by equatorial ocean-atmosphere interaction in the tropical Pacific Ocean (Philander, 1990). The term 'Southern
Oscillation' refers to a tendency for above-average surface atmospheric pressures in the Indian Ocean to be associated with below-average pressures in the Pacific, and vice versa. This oscillation is associated with variations in SSTs in the east equatorial Pacific. The oceanic and atmospheric variations are collectively referred to as ENSO. An El Niño episode is one phase of the ENSO phenomenon and is associated with abnormally warm central and east equatorial Pacific Ocean surface temperatures, while the opposite phase, a La Niña episode, is associated with abnormally cool ocean temperatures in this region. Both phases are associated with a characteristic spatial pattern of droughts and floods. An El Niño episode is usually accompanied by drought in southeastern Asia, India, Australia, southeastern Africa, Amazonia, and northeast Brazil, with fewer than normal tropical cyclones around Australia and in the North Atlantic. Wetter than normal conditions during El Niño episodes are observed along the west coast of tropical South America, subtropical latitudes of western North America, and southeastern America. In a La Niña episode the climate anomalies are usually the opposite of those in an El Niño. Pacific islands are strongly affected by ENSO variations. Recent research (e.g., Kenyon and Hegerl, 2008; Ropelewski and Bell, 2008; Schubert et al., 2008a; Alexander et al., 2009; Grimm and Tedeschi, 2009; Zhang et al., 2010) has demonstrated that different phases of ENSO (El Niño or La Niña episodes) also are associated with different frequencies of occurrence of short-term weather extremes such as heavy rainfall events and extreme temperatures. The relationship between ENSO and interannual variations in tropical cyclone activity is well known (e.g., Kuleshov et al., 2008). The simultaneous occurrence of a variety of climate extremes in an El Niño episode (or a La Niña episode) may provide special challenges for organizations coping with disasters induced by ENSO (see also Section 3.1.1). Monitoring and predicting ENSO can lead to disaster risk reduction through early warning (see Case Study 9.2.11).

The AR4 noted that orbital variations could affect the ENSO behavior (Jansen et al., 2007). Cane (2005) found that a relatively simple coupled model suggested that systematic changes in El Niño could be stimulated by seasonal changes in solar insolation. However, a more comprehensive model simulation (Wittenberg, 2009) has suggested that longterm changes in the behavior of the phenomenon might occur even without forcing from radiative changes. Vecchi and Wittenberg (2010) concluded that the "tropical Pacific could generate variations in ENSO frequency and intensity on its own (via chaotic behavior), respond to external radiative forcings (e.g., changes in greenhouse gases, volcanic eruptions, atmospheric aerosols, etc), or both." Meehl et al. (2009a) demonstrate that solar insolation variations related to the 11-year sunspot cycle can affect ocean temperatures associated with ENSO.

ENSO has varied in strength over the last millennium with stronger activity in the 17th century and late 14th century, and weaker activity during the 12th and 15th centuries (Cobb et al., 2003; Conroy et al., 2009). On longer time scales, there is evidence that ENSO may have changed in response to changes in the orbit of the Earth (Vecchi and Wittenberg, 2010), with the phenomenon apparently being weaker around 6,000 years ago (according to proxy measurements from corals 
and climate model simulations; Rein et al., 2005; Brown et al., 2006; Otto-Bliesner et al., 2009), and model simulations suggest that it was stronger at the last glacial maximum (An et al., 2004). Fossil coral evidence indicates that the phenomenon continued to operate during the last glacial interval (Tudhope et al., 2001). Thus the paleoclimatic evidence indicates that ENSO can continue to operate, although altered perhaps in intensity, in very different background climate states.

The AR4 noted that the nature of ENSO has varied substantially over the period of instrumental data, with strong events from the late 19th century through the first quarter of the 20th century and again after 1950. An apparent climate shift around 1976-1977 was associated with a shift to generally above-normal SSTs in the central and eastern Pacific and a tendency toward more prolonged and stronger El Niño episodes (Trenberth et al., 2007). Ocean temperatures in the central equatorial Pacific (the so-called NINO3 index) suggest a trend toward more frequent or stronger El Niño episodes over the past 50 to 100 years (Vecchi and Wittenberg, 2010). Vecchi et al. (2006) reported a weakening of the equatorial Pacific pressure gradient since the 1960s, with a sharp drop in the 1970s. Power and Smith (2007) proposed that the apparent dominance of El Niño during the last few decades was due in part to a change in the background state of the Southern Oscillation Index (SOI, the standardized difference in surface atmospheric pressure between Tahiti and Darwin), rather than a change in variability or a shift to more frequent El Niño events alone. Nicholls (2008) examined the behavior of the SOI and another index, the NIN03.4 index of central equatorial Pacific SSTs, but found no evidence of trends in the variability or the persistence of the indices [although Yu and Kao (2007) reported decadal variations in the persistence barrier, the tendency for weaker persistence across the Northern Hemisphere spring], nor in their seasonal patterns. There was a trend toward what might be considered more 'El Niño-like' behavior in the SOI (and more weakly in NINO3.4), but only through the period March to September and not in November to February, the season when El Niño and La Niña events typically peak. The trend in the SOI reflected only a trend in Darwin pressures, with no trend in Tahiti pressures. Apart from this trend, the temporal/seasonal nature of ENSO has been remarkably consistent through a period of strong global warming. There is evidence, however, of a tendency for recent El Niño episodes to be centered more in the central equatorial Pacific than in the east Pacific (Yeh et al., 2009), and for these central Pacific episodes to be increasing in intensity (Lee and McPhaden, 2010). In turn, these changes may explain changes that have been noted in the remote influences of the phenomenon on the climate over Australia and in the mid-latitudes (Wang and Hendon, 2007; Weng et al., 2009). For instance, Taschetto et al. (2009) demonstrated that episodes with the warming centered in the central Pacific exhibit different patterns of Australian rainfall variations relative to the east Pacific-centered El Niño events.

The possible role of increased greenhouse gases in affecting the behavior of ENSO over the past 50 to 100 years is uncertain. Yeh et al. (2009) suggested that changes in the background temperature associated with increases in greenhouse gases should affect the behavior of El Niño, such as the location of the strongest SST anomalies, because El Niño behavior is strongly related to the average ocean temperature gradients in the equatorial Pacific. Some studies (e.g., Q. Zhang et al., 2008) have suggested that increased activity might be due to increased $\mathrm{CO}_{2}$; however, no formal attribution study has yet been completed and some other studies (e.g., Power and Smith, 2007) suggest that changes in the phenomenon are within the range of natural variability (i.e., that no change has yet been detected, let alone attributed to a specific cause).

Global warming is projected to lead to a mean reduction in the zonal mean wind across the equatorial Pacific (Vecchi and Soden, 2007b). However, this change should not be described as an 'El Niño-like' average change even though during an El Niño episode these winds also weaken, because there is only limited correspondence between these changes in the mean state of the equatorial Pacific and an El Niño episode. The AR4 determined that all models exhibited continued ENSO interannual variability in projections through the 21 st century, but the projected behavior of the phenomenon differed between models, and it was concluded that "there is no consistent indication at this time of discernible changes in projected ENSO amplitude or frequency in the 21st century" (Meehl et al., 2007b). Models project a wide variety of changes in ENSO variability and the frequency of El Niño episodes as a consequence of increased greenhouse gas concentrations, with a range between a $30 \%$ reduction to a $30 \%$ increase in variability (van Oldenborgh et al., 2005). One model study even found that although ENSO activity increased when atmospheric $\mathrm{CO}_{2}$ concentrations were doubled or quadrupled, a considerable decrease in activity occurred when $\mathrm{CO}_{2}$ was increased by a factor of 16 times, much greater than is possible through the 21st century (Cherchi et al., 2008), suggesting a wide variety of possible ENSO changes as a result of $\mathrm{CO}_{2}$ changes. The remote impacts, on rainfall for instance, of ENSO may change as $\mathrm{CO}_{2}$ increases, even if the equatorial Pacific aspect of ENSO does not change substantially. For instance, regions in which rainfall increases in the future tend to show increases in interannual rainfall variability (Boer, 2009), without any strong change in the interannual variability of tropical SSTs. Also, since some long-term projected changes in response to increased greenhouse gases may resemble the climate response to an El Niño event, this may enhance or mask the response to El Niño events in the future (Lau et al., 2008b; Müller and Roeckner, 2008).

One change that models tend to project is an increasing tendency for El Niño episodes to be centered in the central equatorial Pacific, rather than the traditional location in the eastern equatorial Pacific. Yeh et al. (2009) examined the relative frequency of El Niño episodes simulated in coupled climate models with projected increases in greenhouse gas concentrations. A majority of models, especially those best able to simulate the current ratio of central Pacific locations to east Pacific locations of El Niño events, projected a further increase in the relative frequency of these central Pacific events. Such a change would also have implications for the remote influence of the phenomenon on climate away from the equatorial Pacific (e.g., Australia and India). However, even the projection that the 21st century may see an increased frequency of central Pacific El Niño episodes, relative to the frequency of events located further east (Yeh et al., 2009), is subject to considerable uncertainty. Of 
the 11 coupled climate model simulations examined by Yeh et al. (2009), three projected a relative decrease in the frequency of these central Pacific episodes, and only four of the models produced a statistically significant change to more frequent central Pacific events.

A caveat regarding all projections of future behavior of ENSO arises from systematic biases in the depiction of ENSO behavior through the 20th century by models (Randall et al., 2007; Guilyardi et al., 2009). Leloup et al. (2008) for instance, demonstrate that coupled climate models show wide differences in the ability to reproduce the spatial characteristics of SST variations associated with ENSO during the 20th century, and all models have failings. They concluded that it is difficult to even classify models by the quality of their reproductions of the behavior of ENSO, because models scored unevenly in their reproduction of the different phases of the phenomenon. This makes it difficult to determine which models to use to project future changes in ENSO. Moreover, most of the models are not able to reproduce the typical circulation anomalies associated with ENSO in the Southern Hemisphere (Vera and Silvestri, 2009) and the Northern Hemisphere (Joseph and Nigam, 2006).

There was no consistency in projections of changes in ENSO variability or frequency at the time of the AR4 (Meehl et al., 2007b) and this situation has not changed as a result of post-AR4 studies. The evidence is that the nature of ENSO has varied in the past apparently sometimes in response to changes in radiative forcing but also possibly due to internal climatic variability. Since radiative forcing will continue to change in the future, we can confidently expect changes in ENSO and its impacts as well, although both El Niño and La Niña episodes will continue to occur (e.g., Vecchi and Wittenberg, 2010). Our current limited understanding, however, means that it is not possible at this time to confidently predict whether ENSO activity will be enhanced or damped due to anthropogenic climate change, or even if the frequency of El Niño or La Niña episodes will change (Collins et al., 2010).

In summary, there is medium confidence in a recent trend toward more frequent central equatorial Pacific El Niño episodes, but insufficient evidence for more specific statements about observed trends in ENSO. Model projections of changes in ENSO variability and the frequency of El Niño episodes as a consequence of increased greenhouse gas concentrations are not consistent, and so there is low confidence in projections of changes in the phenomenon. However, there is medium confidence regarding a projected increase (projected by most GCMs) in the relative frequency of central equatorial Pacific events, which typically exhibit different patterns of climate variations than do the classical East Pacific events.

\subsubsection{Other Modes of Variability}

Other natural modes of variability beside ENSO (Section 3.4.2) that are relevant to extremes and disasters include the North Atlantic Oscillation
(NAO), the Southern Annular Mode (SAM), and the Indian Ocean Dipole (IOD) (Trenberth et al., 2007). The NAO is a large-scale seesaw in atmospheric pressure between the subtropical high and the polar low in the North Atlantic region. The positive NAO phase has a strong subtropical high-pressure center and a deeper than normal Icelandic low. This results in a shift of winter storms crossing the Atlantic Ocean to a more northerly track, and is associated with warm and wet winters in northwestern Europe and cold and dry winters in northern Canada and Greenland. Scaife et al. (2008) discuss the relationship between the NAO and European extremes. Paleoclimatic data indicate that the NAO was persistently in its positive phase during medieval times and persistently in its negative phase during the cooler Little Ice Age (Trouet et al., 2009). The NAO is closely related to the Northern Annular Mode (NAM); for brevity we focus here on the NAO but much of what is said about the NAO also applies to the NAM. The SAM is the largest mode of Southern Hemisphere extratropical variability and refers to north-south shifts in atmospheric mass between the middle and high latitudes. It plays an important role in climate variability in these latitudes. The SAM positive phase is linked to negative sea level pressure anomalies over the polar regions and intensified westerlies. It has been associated with cooler than normal temperatures over most of Antarctica and Australia, with warm anomalies over the Antarctic Peninsula, southern South America, and southern New Zealand, and with anomalously dry conditions over southern South America, New Zealand, and Tasmania and wet anomalies over much of Australia and South Africa (e.g., Hendon et al., 2007). The IOD is a coupled ocean-atmosphere phenomenon in the Indian Ocean. A positive IOD event is associated with anomalous cooling in the southeastern equatorial Indian Ocean and anomalous warming in the western equatorial Indian Ocean. Recent work (Ummenhofer et al., 2008, 2009a,b) has implicated the IOD as a cause of droughts in Australia, and heavy rainfall in east Africa (Ummenhofer et al., 2009c). There is also evidence of modes of variability operating on multi-decadal time scales, notably the Pacific Decadal Oscillation (PDO) and the Atlantic Multidecadal Oscillation (AMO). Variations in the PDO have been related to precipitation extremes over North America (Zhang et al., 2010).

Both the NAO and the SAM exhibited trends toward their positive phase (strengthened mid-latitude westerlies) over the last three to four decades, although the NAO has been in its negative phase in the last few years. Goodkin et al. (2008) concluded that the variability in the NAO is linked with changes in the mean temperature of the Northern Hemisphere. Dong et al. (2011) demonstrated that some of the observed late 20thcentury decadal-scale changes in NAO behavior could be reproduced by increasing the $\mathrm{CO}_{2}$ concentrations in a coupled model, and concluded that greenhouse gas concentrations may have played a role in forcing these changes. The largest observed trends in the SAM occur in December to February, and model simulations indicate that these are due mainly to stratospheric ozone changes. However it has been argued that anthropogenic circulation changes are poorly characterized by trends in the annular modes (Woollings et al., 2008). Further complicating these trends, Silvestri and Vera (2009) reported changes in the typical hemispheric circulation pattern related to the SAM and its associated impact on both temperature and precipitation anomalies, particularly 
over South America and Australia, between the 1960s-1970s and 1980s-1990s. The time scales of variability in modes such as the AMO and PDO are so long that it is difficult to diagnose any change in their behavior in modern data, although some evidence suggests that the PDO may be affected by anthropogenic forcing (Meehl et al., 2009b). The AR4 (Hegerl et al., 2007) concluded that trends over recent decades in the NAO and SAM are likely related in part to human activity. The negative NAO phase of the last few years, however, with the lack of formal attribution studies, means that attribution of changes in the NAO to human activity in recent decades now can only be considered about as likely as not (expert opinion). Attribution of the SAM trend to human activity is still assessed to be likely (expert opinion) although mainly attributable to trends in stratospheric ozone concentration (Hegerl et al., 2007).

The AR4 noted that there was considerable spread among the model projections of the NAO, leading to low confidence in NAO projected changes, but the magnitude of the increase for the SAM is generally more consistent across models (Meehl et al., 2007b). However, the ability of coupled models to simulate the observed SAM impact on climate variability in the Southern Hemisphere is limited (e.g., Miller et al., 2006; Vera and Silvestri, 2009). Variations in the longer time-scale modes of variability (AMO, PDO) might affect projections of changes in extremes associated with the various natural modes of variability and global temperatures (Keenlyside et al., 2008).

Sea level pressure is projected to increase over the subtropics and midlatitudes, and decrease over high latitudes (Meehl et al., 2007b). This would equate to trends in the NAO and SAM, with a poleward shift of the storm tracks of several degrees latitude and a consequent increase in cyclonic circulation patterns over the Arctic and Antarctica. In the Southern Hemisphere, two opposing effects, stratospheric ozone recovery and increasing greenhouse gases, can be expected to affect the modes such as the SAM (Arblaster et al., 2011). During the 21st century, although stratospheric ozone concentrations are expected to recover, tending to lead to a weakening of the SAM, models consistently project polar vortex intensification to continue due to the increases in greenhouse gases, except in summer where the competing effects of stratospheric ozone recovery complicate this picture (Arblaster et al., 2011).

A recent study (Woollings et al., 2010) found a tendency toward a more positive NAO under anthropogenic forcing through the 21st century with one model, although they concluded that confidence in the model projections was low because of deficiencies in its simulation of current-day NAO regimes. Goodkin et al. (2008) predict continuing high variability, on multi-decadal scales, in the NAO with continued global warming. Keenlyside et al. (2008) proposed that variations associated with the multi-decadal modes of variability may offset warming due to increased greenhouse gas concentrations over the next decade or so. Conway et al. (2007) reported that model projections of future IOD behavior showed no consistency. Kay and Washington (2008) reported that under some emissions scenarios, changes in a dipole mode in the Indian Ocean could change rainfall extremes in southern Africa.
In summary, it is likely that there has been an anthropogenic influence on recent trends in the SAM (linked with trends in stratospheric ozone rather than changes in greenhouse gases), but it is only about as likely as not that there have been anthropogenic influences on observed trends in the NAO. Issues with the ability of models to simulate current behavior of these natural modes, the influence of competing factors (e.g., stratospheric ozone, greenhouse gases) on current and future mode behavior, and inconsistency between the model projections (and the seasonal dependence of these projections), means that there is low confidence in the ability to project changes in the modes including the NAO, SAM, and IOD. Models do, however, consistently project a strengthening of the polar vortex in the Southern Hemisphere from increasing greenhouse gases, although in summer stratospheric ozone recovery is expected to offset this intensification.

\subsubsection{Tropical Cyclones}

Tropical cyclones occur in most tropical oceans and pose a significant threat to coastal populations and infrastructure, and marine interests such as shipping and offshore activities. Each year, about 90 tropical cyclones occur globally, and this number has remained roughly steady over the modern period of geostationary satellites (since around the mid-1970s). While the global frequency has remained steady, there can be substantial inter-annual to multi-decadal frequency variability within individual ocean basins (e.g., Webster et al., 2005). This regional variability, particularly when combined with substantial inter-annual to multi-decadal variability in tropical cyclone tracks (e.g., Kossin et al., 2010), presents a significant challenge for disaster planning and mitigation aimed at specific regions.

Tropical cyclones are perhaps most commonly associated with extreme wind, but storm-surge and freshwater flooding from extreme rainfall generally cause the great majority of damage and loss of life (e.g., Rappaport, 2000; Webster, 2008). Related indirect factors, such as the failure of the levee system in New Orleans during the passage of Hurricane Katrina (2005), or mudslides during the landfall of Hurricane Mitch (1998) in Central America, represent important related impacts (Case Study 9.2.5). Projected sea level rise will further compound tropical cyclone surge impacts. Tropical cyclones that track poleward can undergo a transition to become extratropical cyclones. While these storms have different characteristics than their tropical progenitors, they can still be accompanied by a storm surge that can impact regions well away from the tropics (e.g., Danard et al., 2004).

Tropical cyclones are typically classified in terms of their intensity, which is a measure of near-surface wind speed (sometimes categorized according to the Saffir-Simpson scale). The strongest storms (Saffir-Simpson category 3,4 , and 5) are comparatively rare but are generally responsible for the majority of damage (e.g., Landsea, 1993; Pielke Jr. et al., 2008) Additionally, there are marked differences in the characteristics of both 
observed and projected tropical cyclone variability when comparing weaker and stronger tropical cyclones (e.g., Webster et al., 2005; Elsner et al., 2008; Bender et al., 2010), while records of the strongest storms are potentially less reliable than those of their weaker counterparts (Landsea et al., 2006).

In addition to intensity, the structure and areal extent of the wind field in tropical cyclones, which can be largely independent of intensity, also play an important role on potential impacts, particularly from storm surge (e.g., Irish and Resio, 2010), but measures of storm size are largely absent in historical data. Other relevant tropical cyclone measures include frequency, duration, and track. Forming robust physical links between all of the metrics briefly mentioned here and natural or human-induced changes in climate variability is a major challenge. Significant progress is being made, but substantial uncertainties still remain due largely to data quality issues (see Section 3.2.1 and below) and imperfect theoretical and modeling frameworks (see below).

\section{Observed Changes}

Detection of trends in tropical cyclone metrics such as frequency, intensity, and duration remains a significant challenge. Historical tropical cyclone records are known to be heterogeneous due to changing observing technology and reporting protocols (e.g., Landsea et al., 2004). Further heterogeneity is introduced when records from multiple ocean basins are combined to explore global trends because data quality and reporting protocols vary substantially between regions (Knapp and Kruk, 2010). Progress has been made toward a more homogeneous global record of tropical cyclone intensity using satellite data (Knapp and Kossin, 2007; Kossin et al., 2007), but these records are necessarily constrained to the satellite era and so only represent the past 30 to 40 years.

Natural variability combined with uncertainties in the historical data makes it difficult to detect trends in tropical cyclone activity. There have been no significant trends observed in global tropical cyclone frequency records, including over the present 40-year period of satellite observations (e.g., Webster et al., 2005). Regional trends in tropical cyclone frequency have been identified in the North Atlantic, but the fidelity of these trends is debated (Holland and Webster, 2007; Landsea, 2007; Mann et al., 2007a). Different methods for estimating undercounts in the earlier part of the North Atlantic tropical cyclone record provide mixed conclusions (Chang and Guo, 2007; Mann et al., 2007b; Kunkel et al., 2008; Vecchi and Knutson, 2008). Regional trends have not been detected in other oceans (Chan and Xu, 2009; Kubota and Chan, 2009; Callaghan and Power, 2011). It thus remains uncertain whether any observed increases in tropical cyclone frequency on time scales longer than about 40 years are robust, after accounting for past changes in observing capabilities (Knutson et al., 2010).

Frequency estimation requires only that a tropical cyclone be identified and reported at some point in its lifetime, whereas intensity estimation requires a series of specifically targeted measurements over the entire duration of the tropical cyclone (e.g., Landsea et al., 2006). Consequently, intensity values in the historical records are especially sensitive to changing technology and improving methodology, which heightens the challenge of detecting trends within the backdrop of natural variability. Global reanalyses of tropical cyclone intensity using a homogenous satellite record have suggested that changing technology has introduced a non-stationary bias that inflates trends in measures of intensity (Kossin et al., 2007), but a significant upward trend in the intensity of the strongest tropical cyclones remains after this bias is accounted for (Elsner et al., 2008). While these analyses are suggestive of a link between observed global tropical cyclone intensity and climate change, they are necessarily confined to a roughly 30 -year period of satellite observations, and cannot provide clear evidence for a longer-term trend.

Time series of power dissipation, an aggregate compound of tropical cyclone frequency, duration, and intensity that measures total energy consumption by tropical cyclones, show upward trends in the North Atlantic and weaker upward trends in the western North Pacific over the past 25 years (Emanuel, 2007), but interpretation of longer-term trends in this quantity is again constrained by data quality concerns. The variability and trend of power dissipation can be related to SST and other local factors such as tropopause temperature and vertical wind shear (Emanuel, 2007), but it is a current topic of debate whether local SST or the difference between local SST and mean tropical SST is the more physically relevant metric (Swanson, 2008). The distinction is an important one when making projections of changes in power dissipation based on projections of SST changes, particularly in the tropical Atlantic where SST has been increasing more rapidly than in the tropics as a whole (Vecchi et al., 2008). Accumulated cyclone energy, which is an integrated metric analogous to power dissipation, has been declining globally since reaching a high point in 2005, and is presently at a $40-$ year low point (Maue, 2009). The present period of quiescence, as well as the period of heightened activity leading up to the high point in 2005, does not clearly represent substantial departures from past variability (Maue, 2009).

Increases in tropical water vapor and rainfall (Trenberth et al., 2005; Lau and $\mathrm{Wu}, 2007)$ have been identified and there is some evidence for related changes in tropical cyclone-related rainfall (Lau et al., 2008a), but a robust and consistent trend in tropical cyclone rainfall has not yet been established due to a general lack of studies. Similarly, an increase in the length of the North Atlantic hurricane season has been noted (Kossin, 2008), but the uncertainty in the amplitude of the trends and the lack of additional studies limits the utility of these results for a meaningful assessment.

Estimates of tropical cyclone variability prior to the modern instrumental historical record have been constructed using archival documents (Chenoweth and Devine, 2008), coastal marsh sediment records, and isotope markers in coral, speleothems, and tree rings, among other methods (Frappier et al., 2007a). These estimates demonstrate centennialto millennial-scale relationships between climate and tropical cyclone 
activity (Donnelly and Woodruff, 2007; Frappier et al., 2007b; Nott et al., 2007; Nyberg et al., 2007; Scileppi and Donnelly, 2007; Neu, 2008; Woodruff et al., 2008a,b; Mann et al., 2009; Yu et al., 2009), but generally do not provide robust evidence that the observed post-industrial tropical cyclone activity is unprecedented.

The AR4 Summary for Policymakers concluded that it is likely that an increase had occurred in intense tropical cyclone activity since 1970 in some regions (IPCC, 2007b). The subsequent CCSP assessment report (Kunkel et al., 2008) concluded that it is likely that the frequency of tropical storms, hurricanes, and major hurricanes in the North Atlantic has increased over the past 100 years, a time in which Atlantic SSTs also increased. Kunkel et al. (2008) also concluded that the increase in Atlantic power dissipation is likely substantial since the 1950s. Based on research subsequent to the AR4 and Kunkel et al. (2008), which further elucidated the scope of uncertainties in the historical tropical cyclone data, the most recent assessment by the World Meteorological Organization (WMO) Expert Team on Climate Change Impacts on Tropical Cyclones (Knutson et al., 2010) concluded that it remains uncertain whether past changes in any tropical cyclone activity (frequency, intensity, rainfall) exceed the variability expected through natural causes, after accounting for changes over time in observing capabilities. The present assessment regarding observed trends in tropical cyclone activity is essentially identical to the WMO assessment (Knutson et al., 2010): there is low confidence that any observed long-term (i.e., 40 years or more) increases in tropical cyclone activity are robust, after accounting for past changes in observing capabilities.

\section{Causes of the Observed Changes}

In addition to the natural variability of tropical SSTs, several studies have concluded that there is a detectable tropical SST warming trend due to increasing greenhouse gases (Karoly and Wu, 2005; Knutson et al., 2006; Santer et al., 2006; Gillett et al., 2008a). The region where this anthropogenic warming has occurred encompasses tropical cyclogenesis regions, and Kunkel et al. (2008) stated that it is very likely that humancaused increases in greenhouse gases have contributed to the increase in SSTs in the North Atlantic and the Northwest Pacific hurricane formation regions over the 20 th century.

Changes in the mean thermodynamic state of the tropics can be directly linked to tropical cyclone variability within the theoretical framework of potential intensity theory (Bister and Emanuel, 1998). In this framework, the expected response of tropical cyclone intensity to observed climate change is relatively straightforward: if climate change causes an increase in the ambient potential intensity that tropical cyclones move through, the distribution of intensities in a representative sample of storms is expected to shift toward greater intensities (Emanuel, 2000; Wing et al., 2007). The fractional changes associated with such a shift in the distribution would be largest in the upper quantiles of the distribution as the strongest tropical cyclones become stronger (Elsner et al., 2008).
Given the evidence that SST in the tropics has increased due to increasing greenhouse gases, and the theoretical expectation that increases in potential intensity will lead to stronger storms, it is essential to fully understand the relationship between SST and potential intensity. Observations demonstrate a strong positive correlation between SST and the potential intensity. This relationship suggests that SST increases will lead to increased potential intensity, which will then ultimately lead to stronger storms (Emanuel, 2000; Wing et al., 2007). However, there is a growing body of research suggesting that local potential intensity is controlled by the difference between local SST and spatially averaged SST in the tropics (Vecchi and Soden, 2007a; Xie et al., 2010; Ramsay and Sobel, 2011). Since increases in SST due to global warming are not expected to lead to continuously increasing SST gradients, this recent research suggests that increasing SST due to global warming, by itself, does not yet have a fully understood physical link to increasingly strong tropical cyclones.

The present period of heightened tropical cyclone activity in the North Atlantic, concurrent with comparative quiescence in other ocean basins (e.g., Maue, 2009), is apparently related to differences in the rate of SST increases, as global SST has been rising steadily but at a slower rate than has the Atlantic (Holland and Webster, 2007). The present period of relatively enhanced warming in the Atlantic has been proposed to be due to internal variability (Zhang and Delworth, 2009), anthropogenic tropospheric aerosols (Mann and Emanuel, 2006), and mineral (dust) aerosols (Evan et al., 2009). None of these proposed mechanisms provide a clear expectation that North Atlantic SST will continue to increase at a greater rate than the tropical mean SST.

Changes in tropical cyclone intensity, frequency, genesis location, duration, and track contribute to what is sometimes broadly defined as 'tropical cyclone activity.' Of these metrics, intensity has the most direct physically reconcilable link to climate variability within the framework of potential intensity theory, as described above (Kossin and Vimont, 2007). Statistical correlations between necessary ambient environmental conditions (e.g., low vertical wind shear and adequate atmospheric instability and moisture) and tropical cyclogenesis frequency have been well documented (DeMaria et al., 2001) but changes in these conditions due specifically to increasing greenhouse gas concentrations do not necessarily preserve the same statistical relationships. For example, the observed minimum SST threshold for tropical cyclogenesis is roughly $26^{\circ} \mathrm{C}$. This relationship might lead to an expectation that anthropogenic warming of tropical SST and the resulting increase in the areal extent of the region of $26^{\circ} \mathrm{C} \mathrm{SST} \mathrm{should} \mathrm{lead} \mathrm{to} \mathrm{increases} \mathrm{in} \mathrm{tropical} \mathrm{cyclone}$ frequency. However, there is a growing body of evidence that the minimum SST threshold for tropical cyclogenesis increases at about the same rate as the SST increase due solely to greenhouse gas forcing (e.g., Ryan et al., 1992; Dutton et al., 2000; Yoshimura et al., 2006; Bengtsson et al., 2007; Knutson et al., 2008; Johnson and Xie, 2010). This is because the threshold conditions for tropical cyclogenesis are controlled not just by surface temperature but also by atmospheric stability (measured from the lower boundary to the tropopause), which responds to greenhouse gas forcing in a more complex way than SST 
alone. That is, when the SST changes due to greenhouse warming are deconvolved from the background natural variability, that part of the SST variability, by itself, has no manifest effect on tropical cyclogenesis. In this case, the simple observed relationship between tropical cyclogenesis and SST, while robust, does not adequately capture the relevant physical mechanisms of tropical cyclogenesis in a warming world.

Another challenge to identifying causes behind observed changes in tropical cyclone activity is introduced by uncertainties in the reanalysis data used to identify environmental changes in regions where tropical cyclones develop and evolve (Bister and Emanuel, 2002; Emanuel, 2010). In particular, heterogeneity in upper-tropospheric kinematic and thermodynamic metrics complicates the interpretation of long-term changes in vertical wind shear and potential intensity, both of which are important environmental controls on tropical cyclones.

Based on a variety of model simulations, the expected long-term changes in global tropical cyclone characteristics under greenhouse warming is a decrease or little change in frequency concurrent with an increase in mean intensity. One of the challenges for identifying these changes in the existing data records is that the expected changes predicted by the models are generally small when compared with changes associated with observed short-term natural variability. Based on changes in tropical cyclone intensity predicted by idealized numerical simulations with $\mathrm{CO}_{2}$-induced tropical SST warming, Knutson and Tuleya (2004) suggested that clearly detectable increases may not be manifest for decades to come. Their argument was based on a comparison of the amplitude of the modeled upward trend (i.e., the signal) in storm intensity with the amplitude of the interannual variability (i.e., the noise). The recent high-resolution dynamical downscaling study of Bender et al. (2010) supports this argument and suggests that the predicted increases in the frequency of the strongest Atlantic storms may not emerge as a clear statistically significant signal until the latter half of the 21st century under the SRES A1B warming scenario. Still, it should be noted that while these model projections suggest that a statistically significant signal may not emerge until some future time, the likelihood of more intense tropical cyclones is projected to continually increase throughout the 21st century.

With the exception of the North Atlantic, much of the global tropical cyclone data is confined to the period from the mid-20th century to present. In addition to the limited period of record, the uncertainties in the historical tropical cyclone data (Section 3.2.1 and this section) and the extent of tropical cyclone variability due to random processes and linkages with various climate modes such as El Niño, do not presently allow for the detection of any clear trends in tropical cyclone activity that can be attributed to greenhouse warming. As such, it remains unclear to what degree the causal phenomena described here have modulated post-industrial tropical cyclone activity.

The AR4 concluded that it is more likely than not that anthropogenic influence has contributed to increases in the frequency of the most intense tropical cyclones (Hegerl et al., 2007). Based on subsequent research that further elucidated the scope of uncertainties in both the historical tropical cyclone data as well as the physical mechanisms underpinning the observed relationships, no such attribution conclusion was drawn in the recent WMO assessment (Knutson et al., 2010). The present assessment regarding detection and attribution of trends in tropical cyclone activity is similar to the WMO assessment (Knutson et al., 2010): the uncertainties in the historical tropical cyclone records, the incomplete understanding of the physical mechanisms linking tropical cyclone metrics to climate change, and the degree of tropical cyclone variability - comprising random processes and linkages to various natural climate modes such as El Niño - provide only low confidence for the attribution of any detectable changes in tropical cyclone activity to anthropogenic influences.

\section{Projected Changes and Uncertainties}

The AR4 concluded (Meehl et al., 2007b) that a broad range of modeling studies project a likely increase in peak wind intensity and near-storm precipitation in future tropical cyclones. A reduction of the overall number of storms was also projected (but with lower confidence), with a greater reduction in weaker storms in most basins and an increase in the frequency of the most intense storms. Knutson et al. (2010) concluded that it is likely that the mean maximum wind speed and near-storm rainfall rates of tropical cyclones will increase with projected 21 stcentury warming, and it is more likely than not that the frequency of the most intense storms will increase substantially in some basins, but it is likely that overall global tropical cyclone frequency will decrease or remain essentially unchanged. The conclusions here are similar to those of the AR4 and Knutson et al. (2010).

The spatial resolution of some models such as the CMIP3 coupled ocean-atmosphere models used in the AR4 is generally not high enough to accurately resolve tropical cyclones, and especially to simulate their intensity (Randall et al., 2007). Higher-resolution global models have had some success in reproducing tropical cyclone-like vortices (e.g., Chauvin et al., 2006; Oouchi et al., 2006; Zhao et al., 2009), but only their coarse characteristics. Significant progress has been recently made, however, using downscaling techniques whereby high-resolution models capable of reproducing more realistic tropical cyclones are run using boundary conditions provided by either reanalysis data sets or output fields from lower-resolution climate models such as those used in the AR4 (e.g., Knutson et al., 2007; Emanuel et al., 2008; Knutson et al., 2008; Emanuel, 2010). A recent study by Bender et al. (2010) applies a cascading technique that downscales first from global to regional scale, and then uses the simulated storms from the regional model to initialize a very high-resolution hurricane forecasting model. These downscaling studies have been increasingly successful at reproducing observed tropical cyclone characteristics, which provides increased confidence in their projections, and it is expected that more progress will be made as computing resources improve. Still, awareness that limitations exist in the models used for tropical cyclone projections, particularly the ability to accurately reproduce natural climate phenomena 
that are known to modulate storm behavior (e.g., ENSO and MJO), is important for context when interpreting model output (Sections 3.2.3.2 and 3.4.2).

While detection of long-term past increases in tropical cyclone activity is complicated by data quality and signal-to-noise issues (as stated above), theory (Emanuel, 1987) and idealized dynamical models (Knutson and Tuleya, 2004) both predict increases in tropical cyclone intensity under greenhouse warming. Recent simulations with highresolution dynamical models (Oouchi et al., 2006; Bengtsson et al., 2007; Gualdi et al., 2008; Knutson et al., 2008; Sugi et al., 2009; Bender et al., 2010) and statistical-dynamical models (Emanuel, 2007) consistently find that greenhouse warming causes tropical cyclone intensity to shift toward stronger storms by the end of the 21 st century (2 to $11 \%$ increase in mean maximum wind speed globally). These and other models also consistently project little change or a reduction in overall tropical cyclone frequency (e.g., Gualdi et al., 2008; Sugi et al., 2009; Murakami et al., 2011), but with an accompanying substantial fractional increase in the frequency of the strongest storms and increased precipitation rates (in the models for which these metrics were examined). Current models project changes in overall global frequency ranging from a decrease of 6 to 34\% by the late 21st century (Knutson et al., 2010). The downscaling experiments of Bender et al. (2010) - which use an 18model ensemble-mean of CMIP3 simulations to nudge a high-resolution dynamical model (Knutson et al., 2008) that is then used to initialize a very high-resolution dynamical model - project a $28 \%$ reduction in the overall frequency of Atlantic storms and an $80 \%$ increase in the frequency of Saffir-Simpson category 4 and 5 Atlantic hurricanes over the next 80 years (A1B scenario).

The projected decreases in global tropical cyclone frequency may be due to increases in vertical wind shear (Vecchi and Soden, 2007c; Zhao et al., 2009; Bender et al., 2010), a weakening of the tropical circulation (Sugi et al., 2002; Bengtsson et al., 2007) associated with a decrease in the upward mass flux accompanying deep convection (Held and Soden, 2006), or an increase in the saturation deficit of the middle troposphere (Emanuel et al., 2008). For individual basins, there is much more uncertainty in projections of tropical cyclone frequency, with changes of up to $\pm 50 \%$ or more projected by various models (Knutson et al., 2010). When projected SST changes are considered in the absence of projected radiative forcing changes, Northern Hemisphere tropical cyclone frequency has been found to increase (Wehner et al., 2010), which is congruent with the hypothesis that SST changes alone do not capture the relevant physical mechanisms controlling tropical cyclogenesis (e.g., Emanuel, 2010).

As noted above, observed changes in rainfall associated with tropical cyclones have not been clearly established. However, as water vapor in the tropics increases (Trenberth et al., 2005) there is an expectation for increased heavy rainfall associated with tropical cyclones in response to associated moisture convergence increases (Held and Soden, 2006). This increase is expected to be compounded by increases in intensity as dynamical convergence under the storm is enhanced. Models in which tropical cyclone precipitation rates have been examined are highly consistent in projecting increased rainfall within the area near the tropical cyclone center under 21st century warming, with increases of 3 to $37 \%$ (Knutson et al., 2010). Typical projected increases are near $20 \%$ within $100 \mathrm{~km}$ of storm centers.

Another type of projection that is sometimes inferred from the literature is based on extrapolation of an observed statistical relationship (see also Section 3.2.3). These relationships are typically constructed on past observed variability that represents a convolution of anthropogenically forced variability and natural variability across a broad range of time scales. In general, however, these relationships cannot be expected to represent all of the relevant physics that control the phenomena of interest, and their extrapolation beyond the range of the observed variability they are built on is not reliable. As an example, there is a strong observed correlation between local SST and tropical cyclone power dissipation (Emanuel, 2007). If 21st-century SST projections are applied to this relationship, power dissipation is projected to increase by about 300\% in the next century (Vecchi et al., 2008; Knutson et al., 2010). Alternatively, there is a similarly strong relationship between power dissipation and relative SST, which represents the difference between local and tropical-mean SST and has been argued to serve as a proxy for local potential intensity (Vecchi and Soden, 2007a). When 21st-century projections of relative SST are considered, this latter relationship projects almost no change in power dissipation in the next century (Vecchi et al., 2006). Both of these statistical relationships can be reasonably defended based on physical arguments but it is not clear which, if either, is correct (Ramsay and Sobel, 2011).

When simulating 21st-century warming under the A1B emission scenario (or a close analog), the present models and downscaling techniques as a whole are consistent in projecting (1) decreases or no change in tropical cyclone frequency, (2) increases in intensity and fractional increases in number of most intense storms, and (3) increases in tropical cyclonerelated rainfall rates. Differences in regional projections lead to lower confidence in basin-specific projections of intensity and rainfall, and confidence is particularly low for projections of frequency within individual basins. More specifically, while projections under 21st-century greenhouse warming indicate that it is likely that the global frequency of tropical cyclones will either decrease or remain essentially unchanged, an increase in mean tropical cyclone maximum wind speed is also likely, although increases may not occur in all tropical regions. This assessment is essentially identical with that of the recent WMO assessment (Knutson et al., 2010). Furthermore, while it is likely that overall global frequency will either decrease or remain essentially unchanged, it is more likely than not that the frequency of the most intense storms (e.g., SaffirSimpson category 4 and 5) will increase substantially in some ocean basins, again agreeing with the recent WMO assessment (Knutson et al., 2010). Based on the level of consistency among models, and physical reasoning, it is likely that tropical cyclone-related rainfall rates will increase with greenhouse warming. Confidence in future projections for particular ocean basins is undermined by the inability of global models to reproduce accurate details at scales relevant to tropical cyclone 
genesis, track, and intensity evolution. Of particular concern is the limited ability of global models to accurately simulate upper-tropospheric wind (Cordero and Forster, 2006; Bender et al., 2010), which modulates vertical wind shear and tropical cyclone genesis and intensity evolution. Thus there is low confidence in projections of changes in tropical cyclone genesis, location, tracks, duration, or areas of impact, and existing model projections do not show dramatic large-scale changes in these features.

In summary, there is low confidence that any observed long-term (i.e., 40 years or more) increases in tropical cyclone activity are robust, after accounting for past changes in observing capabilities. The uncertainties in the historical tropical cyclone records, the incomplete understanding of the physical mechanisms linking tropical cyclone metrics to climate change, and the degree of tropical cyclone variability provide only low confidence for the attribution of any detectable changes in tropical cyclone activity to anthropogenic influences. There is low confidence in projections of changes in tropical cyclone genesis, location, tracks, duration, or areas of impact. Based on the level of consistency among models, and physical reasoning, it is likely that tropical cyclonerelated rainfall rates will increase with greenhouse warming. It is likely that the global frequency of tropical cyclones will either decrease or remain essentially unchanged. An increase in mean tropical cyclone maximum wind speed is likely, although increases may not occur in all tropical regions. While it is likely that overall global frequency will either decrease or remain essentially unchanged, it is more likely than not that the frequency of the most intense storms will increase substantially in some ocean basins.

\subsubsection{Extratropical Cyclones}

Extratropical cyclones (synoptic-scale low-pressure systems) exist throughout the mid-latitudes in both hemispheres and mainly develop over the oceanic basins in the proximity of the upper-tropospheric jet streams, as a result of flow over mountains (lee cyclogenesis) or through conversions from tropical to extratropical systems. It should be noted that regionalized smaller-scale mid-latitude circulation phenomena such as polar lows and mesoscale cyclones are not treated in this section (but see Sections 3.3.3 and 3.4.3). Extratropical cyclones are the main poleward transporter of heat and moisture and may be accompanied by adverse weather conditions such as windstorms, the buildup of waves and storm surges, or extreme precipitation events. Thus, changes in the intensity of extratropical cyclones or a systematic shift in the geographical location of extratropical cyclone activity may have a great impact on a wide range of regional climate extremes as well as the long-term changes in temperature and precipitation. Extratropical cyclones mainly form and grow via atmospheric instabilities such as a disturbance along a zone of strong temperature contrast (baroclinic instabilities), which is a reservoir of available potential energy that can be converted into the kinetic energy associated with extratropical cyclones. Intensification of the cyclones may also take place due to processes such as release of energy due to phase changes of water (latent heat release) (Gutowski et al., 1992; Wernli et al., 2002). Why should we expect climate change to influence extratropical cyclones? A simplified line of argument would be that both the large-scale low and high level pole to equator temperature gradients may change (possibly in opposite directions) in a climate change scenario leading to a change in the atmospheric instabilities responsible for cyclone formation and growth (baroclinicity). These changes may be induced by a variety of mechanisms operating in different parts of the atmospheric column ranging from changing surface conditions (Deser et al., 2007; Bader et al., 2011) to stratospheric changes (Son et al., 2010). In addition, changes in precipitation intensities within extratropical cyclones may change the latent heat release. According to theories on wave-mean flow interaction, changes in the extratropical storm tracks are also associated with changes in the large-scale flow (Robinson, 2000; Lorenz and Hartmann, 2003). A latitudinal shift of the upper tropospheric jet would be accompanied by a latitudinal shift in the extratropical storm track. It is, however, still unclear to what extent a latitudinal shift in the jet changes the total storm track activity rather than shifting it latitudinally (Wettstein and Wallace, 2010). Even within the very simplified outline above the possible impacts of climate change on extratropical cyclone development are many and clearly not trivial.

When validated using reanalyses with similar horizontal resolution, climate models are found to represent the general structure of the storm track pattern well (Bengtsson et al., 2006; Greeves et al., 2007; Ulbrich et al., 2008; Catto et al., 2010). However, using data from five different coupled models, the rate of transfer of zonal available potential energy to eddy available potential energy in synoptic systems was found to be too large, yielding too much energy and an overactive energy cycle (Marques et al., 2011). Models tend to have excessively zonal storm tracks and some show a poor extension of the storm tracks into Europe (Pinto et al., 2006; Greeves et al., 2007; Orsolini and Sorteberg, 2009). It has also been noted that representation of cyclone activity may depend on the physics formulations and the horizontal resolution of the model (Jung et al., 2006; Greeves et al., 2007).

Paleoclimatic proxies for extratropical cyclone variability are still few, but progress is being made in using coastal dune field development and sand grain content of peat bogs as proxies for storminess. Publications covering parts of western Europe indicate enhanced sand movement in European coastal areas during the Little Ice Age (Wilson et al., 2004; de Jong et al., 2006, 2007; Clemmensen et al., 2007; Clarke and Rendell, 2009; Sjogren, 2009). It should be noted that sand influx is also influenced by sediment availability, which is controlled mainly by the degree of vegetation cover and the moisture content of the sediment (Li et al., 2004; Wiggs et al., 2004). Intense cultivation, overgrazing, and forest disturbance make soils more prone to erosion, which can lead to increased sand transport even under less windy conditions. Thus the information gained from paleoclimatic proxies to put the last 100 years of extratropical cyclone variability in context is limited.

Century-long time-series of estimates of extremes in geostrophic wind deduced from triangles of pressure stations, pressure tendencies from 
single stations (see Section 3.3.3 for details), or oceanic variables such as extremes in non-tide residuals are (if these are located in the vicinity of the main storm tracks) possible proxies for extratropical cyclone activity. Trend detection in extratropical cyclone variables such as number of cyclones, intensity, and activity (parameters integrating cyclone intensity, number, and possibly duration) became possible with the development of reanalyses, but remains challenging. Problems with reanalyses have been especially pronounced in the Southern Hemisphere (Hodges et al., 2003; Wang et al., 2006). Even though different reanalyses correspond well in the Northern Hemisphere (Hodges et al., 2003; Hanson et al., 2004), changes in the observing system giving artificial trends in integrated water vapor and kinetic energy (Bengtsson et al., 2004) may have influenced trends in both the number and intensity of cyclones. In addition, studies indicate that the magnitude and even the existence of the changes may depend on the choice of reanalysis (Trigo, 2006; Raible et al., 2008; Simmonds et al., 2008; Ulbrich et al., 2009) and cyclone tracking algorithm (Raible et al., 2008).

The AR4 noted a likely net increase in the frequency/intensity of Northern Hemisphere extreme extratropical cyclones and a poleward shift in the tracks since the 1950s (Trenberth et al., 2007; Table 3.8), and cited several papers showing increases in the number or strength of intense extratropical cyclones both over the North Pacific and the North Atlantic storm track (Trenberth et al., 2007, p. 312) during the last 50 years. Studies using reanalyses indicate a northward and eastward shift in the Atlantic cyclone activity during the last 60 years with both more frequent and more intense wintertime cyclones in the high-latitude Atlantic (Weisse et al., 2005; Wang et al., 2006; Schneidereit et al., 2007; Raible et al., 2008; Vilibic and Sepic, 2010) and fewer in the mid-latitude Atlantic (Wang et al., 2006; Raible et al., 2008). The increase in highlatitude cyclone activity was also reported in several studies of Arctic cyclone activity (X.D. Zhang et al., 2004; Sorteberg and Walsh, 2008; Sepp and Jaagus, 2011). Using ship-based trends in mean sea level pressure (MSLP) variance (which is tied to cyclone intensity), Chang (2007) found wintertime Atlantic trends to be consistent with National Centers for Environmental Prediction (NCEP) reanalysis trends in the Atlantic, but slightly weaker. There are inconsistencies among studies of extreme cyclones in reanalyses, since some studies show an increase in intensity and number of extreme Atlantic cyclones (Geng and Sugi, 2001; Paciorek et al., 2002; Lehmann et al., 2011) while others show a reduction (Gulev et al., 2001). These differences may in part be due to sensitivities of the identification schemes and different definitions of an extreme cyclone (Leckebusch et al., 2006; Pinto et al., 2006). New studies have confirmed that a positive NAM/NAO (see Section 3.4.3) corresponds to stronger Atlantic/European cyclone activity (e.g., Chang, 2009; Pinto et al., 2009; X.L. Wang et al., 2009b). However, studies using long historical records seem to suggest that some of these links may be statistically intermittent (Hanna et al., 2008; Matulla et al., 2008; Allan et al., 2009) due to interdecadal shifts in the location of the positions of the NAO pressure centers (Vicente-Serrano and Lopez-Moreno, 2008; X.D. Zhang et al., 2008). It is unclear to what extent the statistical intermittency implies that the underlying physical processes creating the connection act only intermittently. A possible influence of the Pacific North America (PNA) pattern on the entrance of the North Atlantic storm track (over Newfoundland) has been reported by Pinto et al. (2011). It should be noted that there is some suggestion that the reanalyses cover a time period that starts with relatively low cyclonic activity in northern coastal Europe in the 1960s and reaches a maximum in the 1990s. Long-term European storminess proxies show no clear trends over the last century (Hanna et al., 2008; Allan et al., 2009; see Section 3.3.3 for details).

Studies using reanalyses and in situ data for the last 50 years have noted an increase in the number and intensity of north Pacific wintertime intense extratropical cyclone systems since the 1950s (Graham and Diaz 2001; Simmonds and Keay, 2002; Raible et al., 2008) and cyclone activity (X.D. Zhang et al., 2004), but signs of some of the trends disagreed when different tracking algorithms or reanalysis products were used (Raible et al., 2008). A slight positive trend has been found in north Pacific extreme cyclones (Geng and Sugi, 2001; Gulev et al., 2001; Paciorek et al., 2002). Using ship measurements, Chang (2007) found intensity-related wintertime trends in the Pacific to be about 20 to $60 \%$ of that found in the reanalysis. Long-term in situ observations of north Pacific cyclones based on observed pressure data are considerably fewer than for coastal Europe. However, using hourly tide gauge records from the western coast of the United States as a proxy for storminess, an increasing trend in the extreme winter Non-Tide Residuals (NTR) has been observed in the last decades (Bromirski et al., 2003; Menendez et al., 2008). Years having high NTR were linked to a large-scale atmospheric circulation pattern, with intense storminess associated with a broad, south-easterly displaced, deep Aleutian low that directed storm tracks toward the US West Coast. North Pacific cyclonic activity has been linked to tropical SST anomalies (NINO3.4; see Section 3.4.2) and the PNA (Eichler and Higgins, 2006; Favre and Gershunov, 2006; Seierstad et al., 2007), showing that the PNA and NIN03.4 influence storminess, in particular over the eastern North Pacific with an equatorward shift in storm tracks in the North Pacific basin, as well as an increase in storm track activity along the US East Coast during El Niño events.

Based on reanalyses, North American cyclone numbers have increased over the last 50 years, with no statistically significant change in cyclone intensity (X.D. Zhang et al., 2004). Hourly MSLP data from Canadian stations showed that winter cyclones have become significantly more frequent, longer lasting, and stronger in the lower Canadian Arctic over the last 50 years (1953-2002), but less frequent and weaker in the south, especially along the southeast and southwest Canadian coasts (Wang et al., 2006). Further south, a tendency toward weaker low-pressure systems over the past few decades was found for US East Coast winter cyclones using reanalyses, but no statistically significant trends in the frequency of occurrence of systems (Hirsch et al., 2001).

Studies on extratropical cyclone activity in northern Asia are few. Using reanalyses, a decrease in extratropical cyclone activity (X.D. Zhang et al., 2004) and intensity (X.D. Zhang et al., 2004; X. Wang et al., 2009) over the last 50 years has been reported for northern Eurasia $\left(60-40^{\circ} \mathrm{N}\right)$ with a possible northward shift with increased cyclone frequency in the higher latitudes $\left(50-45^{\circ} \mathrm{N}\right)$ and decrease in the lower latitudes (south of $45^{\circ} \mathrm{N}$ ), 
based on a study with reanalyses. The low-latitude (south of $45^{\circ} \mathrm{N}$ ) decrease was also noted by Zou et al. (2006), who reported a decrease in the number of severe storms for mainland China based on an analysis of extremes of observed 6-hourly pressure tendencies over the last 50 years.

Alexander and Power (2009) showed that the number of observed severe storms at Cape Otway (south-east Australia) has decreased since the mid-19th century, strengthening the evidence of a southward shift in Southern Hemisphere storm tracks previously noted using reanalyses (Fyfe, 2003; Hope et al., 2006; Wang et al., 2006). Frederiksen and Frederiksen (2007) linked the reduction in cyclogenesis at $30^{\circ} \mathrm{S}$ and southward shift to a decrease in the vertical mean meridional temperature gradient. Using reanalyses, both Pezza et al. (2007) and Lim and Simmonds (2009) have confirmed previous studies showing a trend toward more intense low-pressure systems. However, the trend of a decreasing number of cyclones seems to depend on the choice of reanalysis and pressure level (Lim and Simmonds, 2009), emphasizing the weaker consistency among reanalysis products for the Southern Hemisphere extratropical cyclones. Recent studies support the notion of more cyclones around Antarctica when the SAM (see Section 3.4.3) is in its positive phase and a shift of cyclones toward mid-latitudes when the SAM is in its negative phase (Pezza and Simmonds, 2008). Additionally, more intense (and fewer) cyclones seem to occur when the PDO (see Section 3.4.3) is strongly positive and vice versa (Pezza et al., 2007).

In conclusion, it is likely that there has been a poleward shift in the main northern and southern storm tracks during the last 50 years. There is strong agreement with respect to this change between several reanalysis products for a wide selection of cyclone parameters and cyclone identification methods and European and Australian pressurebased storminess proxies are consistent with a poleward shift over the last 50 years, which indicates that the evidence is robust. Advances have been made in documenting the observed decadal and multi-decadal variability of extratropical cyclones using proxies for storminess. So the recent poleward shift should be seen in light of new studies with longer time spans that indicate that the last 50 years coincide with relatively low cyclonic activity in northern coastal Europe in the beginning of the period. Several studies using reanalyses suggest an intensification of high-latitude cyclones, but there is still insufficient knowledge of how changes in the observational systems are influencing the cyclone intensification in reanalyses so even in cases of high agreement among the studies the evidence cannot be considered to be robust, thus we have only low confidence in these changes. Other regional changes in intensity and the number of cyclones have been reported. However, the level of agreement between different studies using different tracking algorithms, different reanalyses, or different cyclone parameters is still low. Thus, we have low confidence in the amplitude, and in some regions in the sign, of the regional changes.

Regarding possible causes of the observed poleward shift, the AR4 concluded that trends over recent decades in the Northern and
Southern Annular Modes, which correspond to sea level pressure reductions over the poles, are likely related in part to human activity, but an anthropogenic influence on extratropical cyclones had not been formally detected, owing to large internal variability and problems due to changes in observing systems (Hegerl et al., 2007). Anthropogenic influences on these modes of variability are also discussed in Section 3.4.3.

Seasonal global sea level pressure changes have been shown to be inconsistent with simulated internal variability (Giannini et al., 2003; Gillett et al., 2005; Gillett and Stott, 2009; X.L. Wang et al., 2009a), but changes in sea level pressure in regions of extratropical cyclones (midand high latitudes) have not formally been attributed to anthropogenic forcings (Gillett and Stott, 2009). However, the trend pattern in atmospheric storminess as inferred from geostrophic wind energy and ocean wave heights has been found to contain a detectable response to anthropogenic and natural forcings with the effect of external forcings being strongest in the winter hemisphere (X.L. Wang et al., 2009a). Nevertheless, the models generally simulate smaller changes than observed and also appear to underestimate the internal variability, reducing the robustness of their detection results. New idealized studies have advanced the physical understanding of how storm tracks may respond to changes in the underlying surface conditions, indicating that a uniform SST increase weakens (reduced cyclone intensity or number of cyclones) and shifts the storm track poleward and strengthened SST gradients near the subtropical jet may lead to a meridional shift in the storm track either toward the poles or the equator depending on the location of the SST gradient change (Deser et al., 2007; Brayshaw et al., 2008; Semmler et al., 2008; Kodama and Iwasaki, 2009), but the average global cyclone activity is not expected to change much under moderate greenhouse gas forcing (O'Gorman and Schneider, 2008; Bengtsson et al., 2009). Studies have also emphasized the important role of stratospheric changes (induced by ozone or greenhouse gas changes) in explaining latitudinal shifts in storm tracks and several mechanisms have been proposed (Son et al., 2010). This has particularly strengthened the understanding of the Southern Hemisphere changes. According to Fogt et al. (2009) both coupled climate models and observed trends in the SAM were found to be outside the range of internal climate variability during the austral summer. This was mainly attributed to stratospheric ozone depletion (see Section 3.4.3).

In summary, there is medium confidence in an anthropogenic influence on the observed poleward shift in extratropical cyclone activity. It has not formally been attributed. However indirect evidence such as global anthropogenic influence on the sea level pressure distribution and trend patterns in atmospheric storminess inferred from geostrophic wind and ocean wave heights has been found. While physical understanding of how anthropogenic forcings may influence extratropical cyclone storm tracks has strengthened, the importance of the different mechanisms in the observed shifts is still unclear.

The AR4 reported that in a future warmer climate, a consistent projection from the majority of the coupled atmosphere-ocean GCMs is fewer 
mid-latitude storms averaged over each hemisphere (Meehl et al., 2007b) and a poleward shift of storm tracks in both hemispheres (particularly evident in the Southern Hemisphere), with greater storm activity at higher latitudes (Meehl et al., 2007b).

A poleward shift in the upper level tropospheric storm track due to increased greenhouse gas forcing is supported by post-AR4 studies (Lorenz and DeWeaver, 2007; 0'Gorman, 2010; Wu et al., 2011). It should be noted that other studies indicate that the poleward shift is less clear when models including a full stratosphere or ozone recovery are used (Huebener et al., 2007; Son et al., 2008; Morgenstern et al., 2010; Scaife et al., 2011) and the strength of the poleward shift is often seen more clearly in upper-level quantities than in low-level transient parameters (Ulbrich et al., 2008). Post-AR4 single model studies support the projection of a reduction in extratropical cyclones averaged over the Northern Hemisphere during future warming (Finnis et al., 2007; Bengtsson et al., 2009; Orsolini and Sorteberg, 2009). However, neither the global changes in storm frequency or intensity were found to be statistically significant by Bengtsson et al. (2009), although they were accompanied by significant increases in total and extreme precipitation.

Models tend to project a reduction of winter cyclone activity throughout the mid-latitude North Pacific and for some models a north-eastern movement of the North Pacific storm track (Loeptien et al., 2008; Ulbrich et al., 2008; Favre and Gershunov, 2009; McDonald, 2011). However, the exact geographical pattern of cyclone frequency anomalies exhibits large variations across models (Teng et al., 2008; Favre and Gershunov, 2009; Laine et al., 2009).

Using band-passed sea level pressure data from 16 CMIP3 coupled GCMs, Ulbrich et al. (2008) showed regional increases in the storm track activity over the Eastern North Atlantic/Western European area. This eastward or southeastward extension of the storm track is also found in other studies (Ulbrich et al., 2008; Laine et al., 2009; McDonald, 2011) and may be attributed to a local minimum in ocean warming in the central North Atlantic and subsequent local changes in baroclinicity (McDonald, 2011). In line with the eastward shift, Donat et al. (2010a) projected an increase in wind storm days for central Europe by the end of the 21st century. The increase varies according to the definition of storminess and one model projects a decrease. A common deficiency among many AR4 models is a coarsely resolved stratosphere and there are still concerns that this may lead to systematic biases in the Atlantic storm track response to increased anthropogenic forcing (Scaife et al., 2011). A reduction in cyclone frequency along the Canadian east coast has been reported (Bengtsson et al., 2006; Watterson, 2006; Pinto et al., 2007a; Teng et al., 2008; Long et al., 2009). New results for Southern Hemisphere cyclones confirm the previously projected poleward shift in storm tracks under increased greenhouse gases (Lim and Simmonds, 2009). That study projected a reduction of Southern Hemisphere extratropical cyclone frequency and intensity in mid-latitudes but a slight increase at high latitudes. The poleward shift due to increased greenhouse gases may be partly opposed by ozone recovery (Son et al., 2010).
Detailed analyses of changes in physical mechanisms related to cyclone changes in coupled climate models are still few. O'Gorman (2010) showed that changes in mean available potential energy of the atmosphere can account for much of the varied response in storm-track intensity to global warming, implying that changes in storm-track intensity are sensitive to competing effects of changes in temperature gradients and static stability in different atmospheric levels. Using two coupled climate models, Laine et al. (2009) indicate that the primary cause for synoptic activity changes at the western end of the Northern Hemisphere storm tracks is related to the baroclinic conversion processes linked to mean temperature gradient changes in localized regions of the western oceanic basins. They also found downstream changes in latent heat release during the developing and mature stages of the cyclone to be of importance and indicated that changes in diabatic process may be amplified by the upstream baroclinic changes [stronger (weaker) baroclinic activity in the west gives stronger (weaker) latent heat release downstream]. Pinto et al. (2009) found that regional increases in track density and intensity of extreme cyclones close to the British Isles using a single model was associated with an eastward shift of the jet stream into Europe, more frequent extreme values of baroclinicity, and stronger upper level divergence.

The modeled reduction in Southern Hemisphere extratropical cyclone frequency and intensity in the mid-latitudes has been attributed to the tropical upper tropospheric warming enhancing static stability and decreasing baroclinicity while an increased meridional temperature gradient in the high latitudes is suggested to be responsible for the increase in cyclone activity in this region (Lim and Simmonds, 2009). In addition to details in the modeled changes in local baroclinicity and diabatic changes, the geographical pattern of modeled response in cyclone activity has been reported to be influenced by the individual model's structure of intrinsic modes of variability (Branstator and Selten, 2009) and biases in the climatology (Kidston and Gerber, 2010).

In summary it is likely that there has been a poleward shift in the main Northern and Southern Hemisphere extratropical storm tracks during the last $\mathbf{5 0}$ years. There is medium confidence in an anthropogenic influence on this observed poleward shift. It has not formally been attributed. There is low confidence in past changes in regional intensity. There is medium confidence that an increased anthropogenic forcing will lead to a reduction in the number of mid-latitude cyclones averaged over each hemisphere, and there is also medium confidence in a poleward shift of the tropospheric storm tracks due to future anthropogenic forcings. Regional changes may be substantial and CMIP3 simulations show some regions with medium agreement. However, there are still uncertainties related to how the poorly resolved stratosphere in many CMIP3 models may influence the regional results. In addition, studies using different analysis techniques, different physical quantities, different thresholds, and different atmospheric vertical levels to represent cyclone activity and storm tracks result in different projections of regional changes. This leads to low confidence in region-specific projections. 


\subsection{Observed and Projected Impacts on the Natural Physical Environment}

\subsubsection{Droughts}

Drought is generally "a period of abnormally dry weather long enough to cause a serious hydrological imbalance" (see the Glossary and Box 3-3). While lack of precipitation (i.e., meteorological drought; Box 3-3) is often the primary cause of drought, increased potential evapotranspiration induced by enhanced radiation, wind speed, or vapor pressure deficit (itself linked to temperature and relative humidity), as well as pre-conditioning (pre-event soil moisture; lake, snow, and/or groundwater storage) can contribute to the emergence of soil moisture and hydrological drought (Box 3-3). Actual evapotranspiration is additionally controlled by soil moisture, which constitutes a limiting factor for further drying under drought conditions, and other processes that impact vegetation development and phenology (e.g., temperature) are also relevant. As noted in the AR4 (Trenberth et al., 2007), there are few direct observations of drought-related variables, in particular of soil moisture, available for a global analysis (see also Section 3.2.1). Hence, proxies for drought ('drought indices') are often used to infer changes in drought conditions. Box 3-3 provides a discussion of the issue of drought definition and a description of commonly used drought indices. In order to understand the impact of droughts (e.g., on crop yields, general ecosystem functioning, water resources, and electricity production), their timing, duration, intensity, and spatial extent need to be characterized. Several weather elements may interact to increase the impact of droughts: enhanced air temperature can indirectly lead to enhanced evaporative demand (through enhanced vapor pressure deficit), although enhanced wind speed or increased incoming radiation are generally more important factors. Moreover, climate phenomena such as monsoons (Section 3.4.1) and ENSO (Section 3.4.2) affect changes in drought occurrence in some

\section{Box 3-3 | The Definition of Drought}

Though a commonly used term, drought is defined in various ways, and these definitional issues make the analysis of changes in drought characteristics difficult. This explains why assessments of (past or projected) changes in drought can substantially differ between published studies or chosen indices (see Section 3.5.1). Some of these difficulties and their causes are highlighted in this box.

\section{What is Drought or Dryness?}

The Glossary defines drought as follows: "A period of abnormally dry weather long enough to cause a serious hydrological imbalance. Drought is a relative term, therefore any discussion in terms of precipitation deficit must refer to the particular precipitation-related activity that is under discussion. For example, shortage of precipitation during the growing season impinges on crop production or ecosystem function in general (due to soil moisture drought, also termed agricultural drought), and during the runoff and percolation season primarily affects water supplies (hydrological drought). Storage changes in soil moisture and groundwater are also affected by increases in actual evapotranspiration in addition to reductions in precipitation. A period with an abnormal precipitation deficit is defined as a meteorological drought. A megadrought is a very lengthy and pervasive drought, lasting much longer than normal, usually a decade or more."

As highlighted in the above definition, drought can be defined from different perspectives, depending on the stakeholders involved. The scientific literature commonly distinguishes meteorological drought, which refers to a deficit of precipitation, soil moisture drought (often called agricultural drought), which refers to a deficit of (mostly root zone) soil moisture, and hydrological drought, which refers to negative anomalies in streamflow, lake, and/or groundwater levels (e.g., Heim Jr., 2002). We use here the term 'soil moisture drought' instead of 'agricultural drought,' despite the widespread use of the latter term (e.g., Heim Jr., 2002; Wang, 2005), because soil moisture deficits have several additional effects beside those on agroecosystems, most importantly on other natural or managed ecosystems (including both forests and pastures), on building infrastructure through soil mechanical processes (e.g., Corti et al., 2009), and health through impacts on heat waves (Section 3.1.4). Water scarcity (linked to socioeconomic drought), which may be caused fully or in part by use from human activities, does not lie within the scope of this chapter (see Section 4.2.2); however, it should be noted that changing pressure on water resources by human uses may itself influence climate and possibly the drought conditions, for example, via declining groundwater levels, or enhanced local evapotranspiration and associated land-atmosphere feedbacks. Drought should not be confused with aridity, which describes the general characteristic of an arid climate (e.g., desert). Indeed, drought is considered a recurring feature of climate occurring in any region and is defined with respect to the average climate of the given region (e.g., Heim Jr., 2002; Dai, 2011). Nonetheless, the effects of droughts are not linear, given the existence of, for example, discrete soil moisture thresholds affecting vegetation and surface fluxes (e.g., Koster et al., 2004b; Seneviratne et al., 2010), which means that the same precipitation deficit or radiation excess relative to normal will not affect different regions equally (e.g., short-term lack of precipitation in a very humid region may not be critical for agriculture because of the ample soil moisture supply). In this chapter we often use the term 'dryness' instead of 'drought' as a more general term. 


\section{Drought Drivers}

For soil moisture or hydrological droughts, the main drivers are reduced precipitation and/or increased evapotranspiration (Figure 3-9). Although the role of deficits in precipitation is generally considered more prominently in the literature, several drought indicators also explicitly or indirectly consider effects of evapotranspiration. In the context of climate projections, analyses suggest that changes in simulated soil moisture drought are mostly driven by changes in precipitation, with increased evapotranspiration from higher vapor pressure deficit (often linked to increased temperature) and available radiation modulating some of the changes (e.g., Burke and Brown, 2008; Sheffield and Wood, 2008a; Orlowsky and Seneviratne, 2011). It should nonetheless be noted that under strong drought conditions, soil moisture becomes limiting for evapotranspiration, thus limits further soil moisture depletion. Other important aspects for soil moisture and hydrological droughts are persistence and pre-conditioning. Because soil moisture, groundwater, and surface waters are associated with water storage, they have a characteristic memory (e.g., Vinnikov et al., 1996; Eltahir and Yeh, 1999; Koster and Suarez, 2001;

Seneviratne et al., 2006b) and thus specific response times to drought forcing (e.g., Begueria et al., 2010; Fleig et al., 2011). The memory

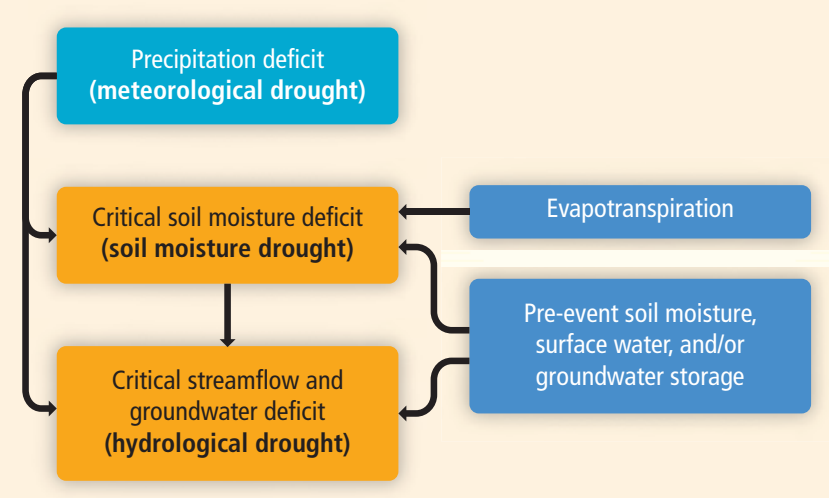

Figure 3-9 | Simplified sketch of processes and drivers relevant for meteorological, soil moisture (agricultural), and hydrological droughts. is also a function of the atmospheric forcing and system's feedbacks (Koster and Suarez, 2001; A.H. Wang et al., 2009), and the relevant storage is dependent on soil characteristics and rooting depth of the considered ecosystems. This means that drought has a different persistence depending on the affected system, and that it is also sensitive to pre-conditioning (Figure 3-9). Effects of pre-conditioning also explain the possible occurrence of multi-year droughts, whereby soil moisture anomalies can be carried over from one year to the next (e.g., Wang, 2005). However, other features can induce drought persistence, such as persistent circulation anomalies, possibly strengthened by land-atmosphere feedbacks (Schubert et al., 2004; Rowell and Jones, 2006). The choice of variable (e.g., precipitation, soil moisture, or streamflow) and time scale can strongly affect the ranking of drought events (Vidal et al., 2010).

\section{Drought Indices}

Because of the complex definition of droughts, and the lack of soil moisture observations (Section 3.2.1), several indices have been developed to characterize (meteorological, soil moisture, and hydrological) drought (see, e.g., Heim Jr., 2002; Dai, 2011). These indicators include land surface, hydrological, or climate model simulations (providing estimates of, e.g., soil moisture or runoff) and indices based on measured meteorological or hydrological variables. We provide here a brief overview of the wide range of drought indices used in the literature for the analysis of recent and projected changes. Note that information on paleoclimate proxies such as tree rings, speleothems, lake sediments, or historical evidence (e.g., harvest dates) is not detailed here.

Some indices are based solely on precipitation data. A widely used index is the Standard Precipitation Index (SPI) (McKee et al., 1993; Lloyd-Hughes and Saunders, 2002), which consists of fitting and transforming a long-term precipitation record into a normal distribution that has zero mean and unit standard deviation. SPI values of -0.5 to -1 correspond to mild droughts, -1 to -1.5 to moderate droughts, -1.5 to -2 to severe droughts, and below -2 to extreme droughts. Similarly, values from 0 to 2 correspond to mildly wet to severely wet conditions, and values above 2 to extremely wet conditions (Lloyd-Hughes and Saunders, 2002). SPI can be computed over several time scales (e.g., 3, 6, 12, or more months) and thus indirectly considers effects of accumulating precipitation deficits, which are critical for soil moisture and hydrological droughts. Another index commonly used in the analysis of climate model simulations is the Consecutive Dry Days (CDD) index, which considers the maximum consecutive number of days without rain (i.e., below a given threshold, typically $1 \mathrm{~mm} \mathrm{day}^{-1}$ ) within a considered period (i.e., year in general; Frich et al., 2002; Alexander et al., 2006; Tebaldi et al., 2006). For seasonal time frames, the CDD periods can either be considered to be bound to the respective seasons (e.g., Figure 3-10) or considered in their entirety (across seasons) but assigned to a specific season. Though SPI and CDD are both only based on precipitation, they do not necessarily only consider the effects of meteorological drought, since periods without rain (thus less cloud cover) are bound to have higher daytime radiation forcing and generally higher temperatures, thus possibly positive evapotranspiration anomalies (unless soil moisture conditions are too dry and limit evapotranspiration).

Some indices reflect both precipitation and estimates of actual or potential evapotranspiration, in some cases also accounting for some temporal accumulation of the forcings or persistence of the drought anomalies. These include the Palmer Drought Severity Index (PDSI) 
(Palmer, 1965), which measures the departure of moisture balance from normal conditions using a simple water balance model (e.g., Dai, 2011), as well as other indices such as the Precipitation Potential Evaporation Anomaly (PPEA, based on the cumulative difference between precipitation and potential evapotranspiration) used in Burke and Brown (2008) and the Standardized PrecipitationEvapotranspiration Index (SPEI, which considers cumulated anomalies of precipitation and potential evapotranspiration) described in Vicente-Serrano et al. (2010). PDSI has been widely used for decades (in particular in the United States), and also in climate change analyses (e.g., Dai et al., 2004; Burke and Brown, 2008; Dai, 2011); however, it has some shortcomings for climate change monitoring and projection. PDSI was originally calibrated for the central United States, which can impair the comparability of the index across regions (and also across time periods if drought mechanisms change over time). Thus it is often of advantage to renormalize the local PDSI (Dai, 2011), which can also be done using the self-calibrated PDSI (Wells et al., 2004), but several studies do not apply these steps. Moreover, the land surface model underlying the computation of the PDSI is essentially a simple bucket-type model, which is less sophisticated than more recent land surface and hydrological models and thus implies several limitations (e.g., Dai et al., 2004; Burke et al., 2006). Another important issue is that the parameterization of potential evapotranspiration as empirically (and solely) dependent on air temperature, which is often applied for these various indices (e.g., in the study of Dai et al., 2004) can lead to biased results (e.g., Donohue et al., 2010; Milly and Dunne, 2011; Shaw and Riha, 2011). Temperature is only an indirect driver of evapotranspiration, via its effect on vapor pressure deficit and via effects on vegetation phenology. Furthermore, approaches using potential evapotranspiration as a proxy for actual evapotranspiration do not consider soil moisture and vegetation control on evapotranspiration, which are important mechanisms limiting drought development.

For the assessment of soil moisture drought, simulated soil moisture anomalies also can be considered (Wang et al., 2005; Burke and Brown, 2008; Sheffield and Wood, 2008a; A.H. Wang et al., 2009; Dai, 2011; Orlowsky and Seneviratne, 2011). Simulated soil moisture anomalies integrate the effects of precipitation forcing, simulated actual evapotranspiration (resulting from atmospheric forcing and simulated soil moisture limitation on evapotranspiration), and simulated soil moisture persistence. Although the soil moisture simulated by (land-surface, hydrological, and climate) models often exhibits strong discrepancies in absolute terms, soil moisture anomalies can be compared with simple scaling and generally match reasonably well (e.g., Koster et al., 2009; A.H. Wang et al., 2009). Soil moisture persistence is found to be an important component in projected changes in soil moisture drought, with some regions displaying yearround dryness compared to reference (late 20th or pre-industrial) conditions due to the carry-over effect of soil moisture storage from season to season, leading to year-round soil moisture deficits compared to late 20th century climate (e.g., Wang et al., 2005, Figure 3-10). However, it should be noted that some land surface and hydrological models (used offline or coupled to climate models) suffer from similar shortcomings as noted above for PDSI - that is, they use simple bucket models or simplified representations of potential evapotranspiration. The latter issue has been suggested as being particularly critical for models used in offline mode (Milly and Dunne, 2011). Nonetheless, for the assessment of soil moisture drought, using simulated soil moisture anomalies seems less problematic than many other indices for the reasons highlighted in the above paragraphs.

The indices listed above have been used in various studies analyzing drought in the context of climate change, but with a few exceptions most available studies are based only on one index, which makes their comparison difficult. Nonetheless, these studies suggest that projections can be highly dependent on the choice of drought index. For instance, one study projected changes in drought area possibly varying between a negligible impact and a 5 to $45 \%$ increase depending on the drought index considered (Burke and Brown, 2008). Other drought indices are used to quantify hydrological drought (e.g., Heim Jr., 2002; Vidal et al., 2010; Dai, 2011), but are less commonly used in climate change studies. Further analyses or indices also consider the area affected by droughts (e.g., Burke et al., 2006; Sheffield and Wood, 2008a; Dai, 2011) or additional variables (such as snow or vegetation indices from satellite measurements, e.g., Heim Jr., 2002). As for the definition of other indices (Box 3-1), the determination of the reference period is critical for the assessment of changes in drought patterns independently of the chosen index. In general, late 20th-century conditions are used as reference (e.g., Figure 3-10).

In summary, drought indices often integrate precipitation, temperature, and other variables, but may emphasize different aspects of drought and should be carefully selected with respect to the drought characteristic in mind. In particular, some indices have specific shortcomings, especially in the context of climate change. For this reason, assessments of changes in drought characteristics with climate change should consider several indices including a specific evaluation of their relevance to the addressed question to support robust conclusions. In this assessment we focus on the following indices: consecutive dry days (CDD) and simulated soil moisture anomalies (SMA), although evidence based on other indices (e.g., PDSI for present climate) is also considered (Section 3.5.1; Tables 3-2 and 3-3). 
regions. Hence, drought is a complex phenomenon that is strongly affected by other extremes considered in this chapter, but that is also affected by changes in mean climate features (Section 3.1.6). In addition, via land-atmosphere interactions, drought also has the potential to impact other weather and climate elements such as temperature and precipitation and associated extremes (Koster et al., 2004b; Seneviratne et al., 2006a; Hirschi et al., 2011; see also Section 3.1.4). Case Study 9.2.3 addresses aspects related to the management of adverse consequences of droughts; while Case Study 9.2.2 considers the possible impacts of high temperatures and drought on wildfire.

\section{Observed Changes}

There are still large uncertainties regarding observed global-scale trends in droughts. The AR4 reported based on analyses using PDSI (see Box 3-3) that very dry areas had more than doubled in extent since 1970 at the global scale (Trenberth et al., 2007). This assessment was, however, largely based on the study by Dai et al. (2004) only. These trends in the PDSI proxy were found to be largely affected by changes in temperature, not precipitation (Dai et al., 2004). On the other hand, based on soil moisture simulations with an observation-driven land surface model for the time period 1950-2000, Sheffield and Wood (2008a) have inferred trends in drought duration, intensity, and severity predominantly decreasing, but with strong regional variation and including increases in some regions. They concluded that there was an overall moistening trend over the considered time period, but also a switch since the 1970s to a drying trend, globally and in many regions, especially in high northern latitudes. Some regional studies are consistent with the results from Sheffield and Wood (2008a), regarding, for example, less widespread increase (or statistically insignificant changes or decreases) in some regions compared to the study of Dai et al. (2004) (e.g., in Europe, see below). More recently, Dai (2011) by extending the record did, however, find widespread increases in drought both based on various versions of PDSI (for 1950-2008) and soil moisture output from a land surface model (for 1948-2004). Hence there are still large uncertainties with respect to global assessments of past changes in droughts. Nonetheless, there is some agreement between studies over the different time frames (i.e., since 1950 versus 1970) and using different drought indicators regarding increasing drought occurrence in some regions (e.g., southern Europe, West Africa; see below and Table 3-2), although other regions also indicate opposite trends (e.g., central North America, northwestern Australia; see below and Table 3-2). As mentioned in Section 3.1.6, spatially coherent shifts in drought regimes are expected with changing global circulation patterns. Table 3-2 provides regional and continental-scale assessments of observed trends in dryness based on different indices (Box 3-3). The following paragraphs provide more details by continent.

From a paleoclimate perspective recent droughts are not unprecedented, with severe 'megadroughts' reported in the paleoclimatic record for Europe, North America, and Australia (Jansen et al., 2007). Recent studies extend this observation to African and Indian droughts (Sinha et al., 2007; Shanahan et al., 2009): much more severe and longer droughts occurred in the past centuries with widespread ecological, political, and socioeconomic consequences. Overall, these studies confirm that in the last millennium several extreme droughts have occurred (Breda and Badeau, 2008; Kallis, 2008; Büntgen et al., 2010).

In North America, there is medium confidence that there has been an overall slight tendency toward less dryness (wetting trend with more soil moisture and runoff; Table 3-2), although analyses for some subregions also indicate tendencies toward increasing dryness. This assessment is based on several lines of evidence, including simulations with different hydrological models as well as PDSI and CDD estimates (Alexander et al., 2006; Andreadis and Lettenmaier, 2006; van der Schrier et al., 2006a; Kunkel et al., 2008; Sheffield and Wood, 2008a; Dai, 2011). The most severe droughts in the 20th century have occurred in the 1930s and 1950s, where the 1930s Dust Bowl was most intense and the 1950s drought most persistent (Andreadis et al., 2005) in the United States, while in Mexico the 1950s and late 1990s were the driest periods. Recent regional trends toward more severe drought conditions were identified over southern and western Canada, Alaska, and Mexico, with subregional exceptions (Dai, 2011).

In Europe, there is medium confidence regarding increases in dryness based on some indices in the southern part of the continent, but large inconsistencies between indices in this region, and inconsistent or statistically insignificant trends in the rest of the continent (Table 3-2). Although Dai et al. (2004) found an increase in dryness for most of the European continent based on PDSI, Lloyd-Hughes and Saunders (2002) and van der Schrier et al. (2006b) concluded, based on the analysis of SPI and self-calibrating PDSI for the 20th century (for 1901-1999 and 1901-2002, respectively), that no statistically significant changes were observed in extreme and moderate drought conditions in Europe [with the exception of the Mediterranean region in van der Schrier et al. (2006b)]. Sheffield and Wood (2008a) also found contrasting dryness trends in Europe, with increases in the southern and eastern part of the continent, but decreases elsewhere. Beniston (2009b) reported a strong increase in warm-dry conditions over all central-southern (including maritime) Europe via a quartile analysis from the middle to the end of the 20th century. Alexander et al. (2006) found trends toward increasing CDD mostly in the southern and central part of the continent. Trends of decreasing precipitation and discharge are consistent with increasing salinity in the Mediterranean Sea, indicating a trend toward freshwater deficits (Mariotti et al., 2008), but this could also be partly caused by increased human water use. In France, an analysis based on a variation of the PDSI model also reported a significant increasing trend in drought conditions, in particular from the 1990s onward (Corti et al., 2009). Stahl et al. (2010) investigated streamflow data across Europe and found negative trends (lower streamflow) in southern and eastern regions, and generally positive trends (higher streamflow) elsewhere (especially in northern latitudes). Low flows have decreased in most regions where the lowest mean monthly flow occurs in summer, but vary for catchments that have flow minima in winter and secondary low flows in summer. The exceptional 2003 summer heat wave on the European continent (see Section 3.3.1) was also associated with a 
major soil moisture drought, as could be inferred from satellite measurements (Andersen et al., 2005), model simulations (Fischer et al., 2007a,b), and impacts on ecosystems (Ciais et al., 2005; Reichstein et al., 2007).

There is low confidence in dryness trends in South America (Table 3-2), partly due to lack of data and partly due to inconsistencies. For the Amazon, repeated intense droughts have been occurring in the last decades but no particular trend has been reported. The 2005 and 2010 droughts in Amazonia are, however, considered the strongest in the last century as inferred from integrating precipitation records and water storage estimates via satellite (measurements from the Gravity Recovery and Climate Experiment; Chen et al., 2009; Lewis et al., 2011). For other parts of South America, analyses of the return intervals between droughts in the instrumental and reconstructed precipitation series indicate that the probability of drought has increased during the late 19th and 20th centuries, consistent with selected long instrumental precipitation records and with a recession of glaciers in the Chilean and Argentinean Andean Cordillera (Le Quesne et al., 2006, 2009).

Changes in drought patterns have been reported for the monsoon regions of Asia and Africa with variations at the decadal time scale (e.g., Janicot, 2009). In Asia there is overall low confidence in trends in dryness both at the continental and regional scale, mostly due to spatially varying trends, except in East Asia where a range of studies, based on different indices, show increasing dryness in the second half of the 20th century, leading to medium confidence (Table 3-2).

In the Sahel, recent years have been characterized by greater interannual variability than the previous 40 years (Ali and Lebel, 2009; Greene et al., 2009), and by a contrast between the western Sahel remaining dry and the eastern Sahel returning to wetter conditions (Ali and Lebel, 2009). Giannini et al. (2008) report a drying of the African monsoon regions, related to warming of the tropical oceans, and variability related to ENSO. In the different subregions of Africa there is overall low to medium confidence regarding regional dryness trends (Table 3-2).

For Australia, Sheffield and Wood (2008a) found very limited increases in dryness from 1950 to 2000 based on soil moisture simulated using existing climate forcing (mostly in southeastern Australia) and some marked decreases in dryness in central Australia and the northwestern part of the continent. Dai (2011), for an extended period until 2008 and using different PDSI variants as well as soil moisture output from a land surface model, found a more extended drying trend in the eastern half of the continent, but also a decrease in dryness in most of the western half. Jung et al. (2010) inferred from a combination of remote sensing and quasi-globally distributed eddy covariance flux observations that in particular the decade after 1998 became drier in Australia (and parts of Africa and South America), leading to decreased evapotranspiration, but it is not clear if this is a trend or just decadal variation.

Following the assessment of observed changes in the AR4 (Chapter 3), which was largely based on one study (Dai et al., 2004), subsequent work has drawn a more differentiated picture both regionally and temporally. There is not enough evidence at present to suggest high confidence in observed trends in dryness due to lack of direct observations, some geographical inconsistencies in the trends, and some dependencies of inferred trends on the index choice. There is medium confidence that since the 1950s some regions of the world have experienced more intense and longer droughts (e.g., southern Europe, west Africa) but also opposite trends exist in other regions (e.g., central North America, northwestern Australia).

\section{Causes of the Observed Changes}

The AR4 (Hegerl et al., 2007) concluded that it is more likely than not that anthropogenic influence has contributed to the increase in the droughts observed in the second half of the 20th century. This assessment was based on several lines of evidence, including a detection study that identified an anthropogenic fingerprint in a global PDSI data set with high significance (Burke et al., 2006), although the model trend was weaker than observed and the relative contributions of natural external forcings and anthropogenic forcings were not assessed.

There is now a better understanding of the potential role of landatmosphere feedbacks versus SST forcing for meteorological droughts (e.g., Schubert et al., 2008a,b), and some modeling studies have also addressed potential impacts of land use changes (e.g., Deo et al., 2009), but large uncertainties remain in the field of land surface modeling and land-atmosphere interactions, in part due to lack of observations (Seneviratne et al., 2010), inter-model discrepancies (Koster et al., 2004b; Dirmeyer et al., 2006; Pitman et al., 2009), and model resolution of orographic and other effects. Nonetheless, a new set of climate modeling studies show that US drought response to SST variability is consistent with observations (Schubert et al., 2009). Inferred trends in drought are also consistent with trends in global precipitation and temperature, and the latter two are consistent with expected responses to anthropogenic forcing (Hegerl et al., 2007; X. Zhang et al., 2007). The change in the pattern of global precipitation in the observations and in model simulations is also consistent with the theoretical understanding of hydrological response to global warming that wet regions become overall wetter and dry regions drier in a warming world (Held and Soden, 2006; see also Section 3.1.6), though some regions also display shifts in climate regimes (Section 3.1.6). Nonetheless, some single events have been reported as differing from projections (Seager et al., 2009), though this is not necessarily incompatible given the superimposition of anthropogenic climate change and natural climate variability (Section 3.1). For soil moisture and hydrological drought it has been suggested that the stomatal 'antitranspirant' responses of plants to rising atmospheric $\mathrm{CO}_{2}$ may lead to a decrease in evapotranspiration (Gedney et al., 2006). This could mean that increasing $\mathrm{CO}_{2}$ levels alleviate soil moisture and streamflow drought, but this result is still debated (e.g., Piao et al., 2007; Gerten et al., 2008), in particular due to the uncertainty in observed runoff trends used to infer these effects (e.g., Peel and McMahon, 2006; see also Section 3.2.1). 
Overall, though new studies have furthered the understanding of the mechanisms leading to drought, there is still relatively limited evidence to provide an attribution of observed changes, in particular given the issues associated with the availability of observational data (Section 3.2.1) and the definition and computation of drought indicators (Box 3-3). This latter point was mostly identified in post-AR4 studies (Box 3-3). Moreover, regions where consistent increases in drought are identified (see 'Observed Changes') are only partly consistent with those where projections indicate an enhancement of drought conditions in coming decades (see next paragraphs). We thus assess that there is medium confidence (see also Section 3.1.5) that anthropogenic influence has contributed to some changes in the drought patterns observed in the second half of the 20th century, based on its attributed impact on precipitation and temperature changes (though temperature can only be indirectly related to drought trends; see Box 3-3). However there is low confidence in the attribution of changes in droughts at the level of individual regions.

\section{Projected Changes and Uncertainties}

The AR4 assessed that projections at the time indicated an increase in droughts, in particular in subtropical and mid-latitude areas (Christensen et al., 2007). An increase in dry spell length and frequency was considered very likely over the Mediterranean region, southern areas of Australia, and New Zealand and likely over most subtropical regions, with little change over northern Europe. Continental drying and the associated risk of drought were considered likely to increase in summer over many mid-latitude continental interiors (e.g., central and southern Europe, the Mediterranean region), in boreal spring, and dry periods of the annual cycle over Central America.

More recent global and regional climate simulations and hydrological models mostly support the projections from the AR4, as summarized in the following paragraphs (see also Table 3-3), although we assess the overall confidence in drought projections as medium given the definitional issues associated with dryness and the partial lack of agreement in model projections when based on different dryness indices (Box 3-3). Indeed, particular care is needed in inter-comparing 'drought' projections since very many different definitions are employed (corresponding to different types of droughts), from simple climatic indices such as CDD to more complex indices of soil moisture and hydrological drought (Box 3-3). A distinction also needs to be made between short-term and longer-term events. Blenkinsop and Fowler (2007a) and Burke et al. (2010), for example, show different trend strength, and sometimes sign (Blenkinsop and Fowler, 2007a), for changes in short- and long-term droughts with RCM ensembles applied to the United Kingdom (although uncertainties in the latter projections are large; see below). These various distinctions are generally not considered and most currently available studies only assess changes in very few (most commonly one or two) dryness indices.

On the global scale, Burke and Brown (2008) provided an analysis of projected changes in drought based on four indices (SPI, PDSI, PPEA, and SMA; for definitions, see Box 3-3) using two model ensembles: one based on a GCM expressing uncertainty in parameter space, and a multimodel ensemble of 11 GCM simulations from CMIP3. Their analysis revealed that SPI, based solely on precipitation, showed little change in the proportion of the land surface in drought, and that all other indices, which include a measure of the atmospheric demand for moisture, showed a statistically significant increase with an additional 5 to $45 \%$ of the land surface in drought. This study also highlighted large uncertainties in regional changes in drought. For reasons highlighted in Box 3-3, using simulated soil moisture anomalies from the climate models avoids some shortcomings of other commonly used indices (although the quality of simulated soil moisture cannot be well evaluated due to lack of observations; Section 3.2 and Box 3-3). In the study of Burke and Brown (2008), this index showed weaker drying compared to PDSI and PPEA indices (but more pronounced drying than the SPI index). In this report, we display projected changes in soil moisture anomalies and CDD (Figure 3-10), this latter index being chosen for continuity with the AR4 (see Figure 10.18 of that report). It can be seen that the two indices partly agree on increased drought in some large regions (e.g., on the annual time scale, in Southern Europe and the Mediterranean region, central Europe, central North America, Central America and Mexico, northeast Brazil, and southern Africa), but some regions where the models show consistent increases in CDD (e.g., southeast Asia) do not show consistent decreases in soil moisture. Conversely, regions displaying a consistent decrease in CDD (e.g., in northeastern Asia) do not show a consistent increase in soil moisture. The substantial uncertainty of drought projections is particularly clear from the soil moisture projections, with, for example, no agreement among the models regarding the sign of changes in December to February over most of the globe. These results regarding changes in CDD and soil moisture are consistent with other published studies (Wang, 2005; Tebaldi et al., 2006; Burke and Brown, 2008; Sheffield and Wood, 2008b; Sillmann and Roeckner, 2008) and the areas that display consistent increasing drought tendencies for both indices have also been reported to display such tendencies for additional indices (e.g., Burke and Brown, 2008; Dai, 2011; Table 3-3). Sheffield and Wood (2008b) examined projections in drought frequency (for droughts of duration of 4 to 6 months and longer than 12 months, estimated from soil moisture anomalies) based on CMIP3 simulations with eight GCMs and the SRES scenarios $A 2, A 1 B$, and B1. They concluded that drought was projected to increase in several regions under these three scenarios (mostly consistent with those displayed in Figure 3-10 for SMA), although the projections of drought intensification were stronger for the high $\mathrm{CO}_{2}$ emissions scenarios ( $\mathrm{A} 2$ and $\mathrm{A} 1 \mathrm{~B}$ ) than for the more moderate scenario (B1). Regions showing statistically significant increases in drought frequency were found to be broadly similar for all three scenarios, despite the more moderate signal in the B1 scenario (their Figures 8 and 9). This study also highlighted the large uncertainty of scenarios for drought projections, as scenarios were found to span a large range of changes in drought frequency in most regions, from close to no change to two- to three-fold increases (their Figure 10).

Regional climate simulations and high-resolution global atmospheric model simulations over Europe also highlight the Mediterranean region 


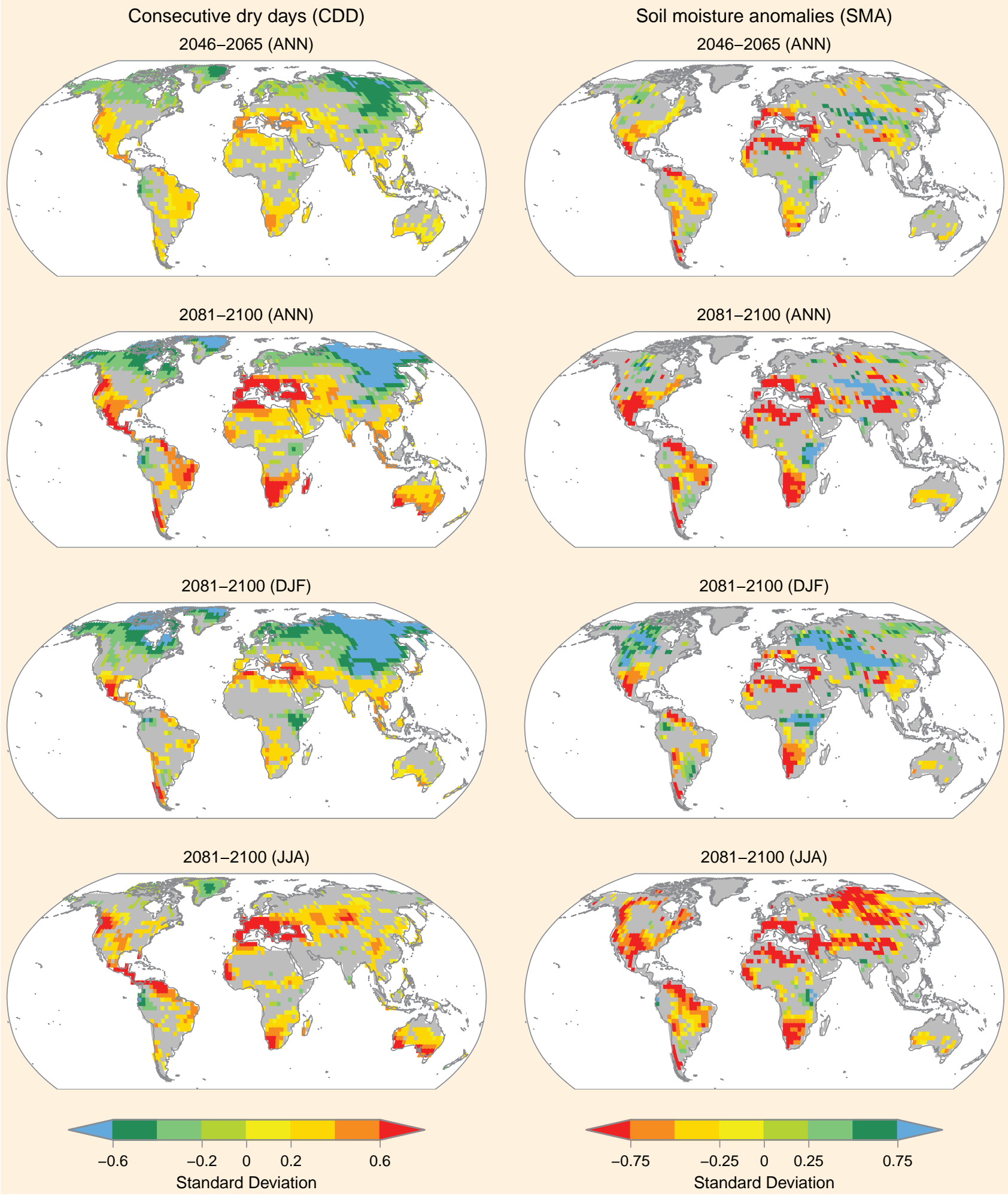

Figure 3-10 | Projected annual and seasonal changes in dryness assessed from two indices for 2081-2100 (bottom three rows, showing the annual time scale and two seasons, DJF and JJA) and 2046-2065 (top, annual time scale) with respect to 1980-1999. Left column: changes in the maximum number of CDD (days with precipitation $<1 \mathrm{~mm}$ ), based on $17 \mathrm{GCMs}$ contributing to the CMIP3. Right column: changes in soil moisture (soil moisture anomalies, SMA), based on 15 GCMs contributing to the CMIP3. Increased dryness is indicated with warm colors (positive changes in CDD and negative SMA values). The maps show differences between the annual and seasonal averages over the respective 20-year periods, that is, the average of 2081-2100 or 2046-2065, respectively (based on simulations under emission scenario SRES A2), minus the average of 1980-1999 (from corresponding simulations for the 20th century). Differences are expressed in units of standard deviations, derived from detrended per year annual or seasonal estimates, respectively, from the three 20-year periods 1980-1999, 2046-2065, and 2081-2100 pooled together. Color shading is only applied for areas where at least $66 \%$ of the GCMs (12 out of 17 for CDD, 10 out of 15 for soil moisture) agree on the sign of the change; stippling is applied for regions where at least $90 \%$ of the GCMs (16 out of 17 for CDD, 14 out of 15 for soil moisture) agree on the sign of the change. Adapted from Orlowsky and Seneviratne (2011); updating Tebaldi et al. (2006) for SMA and for additional CMIP3 models, and including seasonal time frames. For more details, see Appendix 3.A. 
as being affected by more severe droughts, consistent with available global projections (Table 3-3; see also Giorgi, 2006; Rowell and Jones, 2006; Beniston et al., 2007; Mariotti et al., 2008; Planton et al., 2008). Mediterranean (summer) droughts are projected to start earlier in the year and last longer. Also, increased variability during the dry and warm season is projected (Giorgi, 2006). One GCM-based study projected one to three weeks of additional dry days for the Mediterranean region by the end of the century (Giannakopoulos et al., 2009). For North America, intense and heavy episodic rainfall events with high runoff amounts are interspersed with longer relatively dry periods with increased evapotranspiration, particularly in the subtropics. There is a consensus of most climate model projections for a reduction in cool season precipitation across the US southwest and northwest Mexico (Christensen et al., 2007), with more frequent multi-year drought in the US southwest (Seager et al., 2007; Cayan et al., 2010). Reduced cool season precipitation promotes drier summer conditions by reducing the amount of soil water available for evapotranspiration in summer. For Australia, Alexander and Arblaster (2009) project increases in consecutive dry days, although consensus between models is only found in the interior of the continent. African studies indicate the possibility of relatively small-scale $(500-\mathrm{km})$ heterogeneity of changes in precipitation and drought, based on climate model simulations (Funk et al., 2008; Shongwe et al., 2009). Regional climate simulations of South America project spatially coherent increases in CDD, particularly large over the Brazilian Plateau, and northern Chile and the Altiplano (Kitoh et al., 2011).

Available global and regional studies of hydrological drought (Hirabayashi et al., 2008b; Feyen and Dankers, 2009) project a higher likelihood of hydrological drought by the end of this century, with a substantial increase in the number of drought days (defined as streamflow below a specific threshold) during the last 30 years of the 21st century over North and South America, central and southern Africa, the Middle East, southern Asia from Indochina to southern China, and central and western Australia. Some regions, including eastern Europe to central Eurasia, inland China, and northern North America, project increases in drought. In contrast, wide areas over eastern Russia project a decrease in drought days. At least in Europe, hydrological drought is primarily projected to occur in the frost-free season.

Increased confidence in modeling drought stems from consistency between models and satisfactory simulation of drought indices during the past century (Sheffield and Wood, 2008a; Sillmann and Roeckner, 2008). Inter-model agreement is stronger for long-term droughts and larger spatial scales (in some regions, see above discussion), while local to regional and short-term precipitation deficits are highly spatially variable and much less consistent between models (Blenkinsop and Fowler, 2007b). Insufficient knowledge of the physical causes of meteorological droughts, and of the links to the large-scale atmospheric and ocean circulation, is still a source of uncertainty in drought simulations and projections. For example, plausible explanations have been proposed for projections of both a worsening drought and a substantial increase in rainfall in the Sahara (Biasutti et al., 2009; Burke et al., 2010). Another example is illustrated with the relationship of rainfall in southern
Australia with SSTs around northern Australia. On annual time scales, low rainfall is associated with cooler than normal SSTs. Yet the warming observed in SST over the past few decades has not been associated with increased rainfall, but with a trend toward more drought-like conditions (N. Nicholls, 2010).

There are still further sources of uncertainties affecting the projections of trends in meteorological drought for the coming century. The two most important may be uncertainties in the development of the ocean circulation and feedbacks between land surface and atmospheric processes. These latter processes are related to the effects of drought on vegetation physiology and dynamics (e.g., affecting canopy conductance, albedo, and roughness), with resulting (positive or negative) feedbacks to precipitation formation (Findell and Eltahir, 2003a,b; Koster et al., 2004b; Cook et al., 2006; Hohenegger et al., 2009; Seneviratne et al., 2010; van den Hurk and van Meijgaard, 2010), and possibly - as only recently highlighted - also feedbacks between droughts, fires, and aerosols (Bevan et al., 2009). Furthermore, the development of soil moisture that results from complex interactions among precipitation, water storage as soil moisture (and snow), and evapotranspiration by vegetation is still associated with large uncertainties, in particular because of lack of observations of soil moisture and evapotranspiration (Section 3.2.1), and issues in the representation of soil moistureevapotranspiration coupling in current climate models (Dirmeyer et al., 2006; Seneviratne et al., 2010). Uncertainties regarding soil moistureclimate interactions are also due to uncertainties regarding the behavior of plant transpiration, growth, and water use efficiency under enhanced atmospheric $\mathrm{CO}_{2}$ concentrations, which could potentially have impacts on the hydrological cycle (Betts et al., 2007), but are not well understood yet (Hungate et al., 2003; Piao et al., 2007; Bonan, 2008; Teuling et al., 2009; see also above discussion on the causes of observed changes). The space-time development of hydrological drought as a response to a meteorological drought and the associated soil moisture drought (drought propagation, e.g., Peters et al., 2003) also needs more attention. There is some understanding of these issues at the catchment scale (e.g., Tallaksen et al., 2009), but these need to be extended to the regional and continental scales. This would lead to better understanding of the projections of hydrological droughts, which would contribute to a better identification and attribution of droughts and help to improve global hydrological models and land surface models.

In summary, there is medium confidence that since the $1950 \mathrm{~s}$ some regions of the world have experienced trends toward more intense and longer droughts, in particular in southern Europe and West Africa, but in some regions droughts have become less frequent, less intense, or shorter, for example, central North America and northwestern Australia. There is medium confidence that anthropogenic influence has contributed to some changes in the drought patterns observed in the second half of the 20th century, based on its attributed impact on precipitation and temperature changes (though temperature can only be indirectly related to drought trends; see Box 3-3). However there is low confidence in the attribution of changes in droughts at the level 
of single regions due to inconsistent or insufficient evidence. Post-AR4 studies indicate that there is medium confidence in a projected increase in duration and intensity of droughts in some regions of the world, including southern Europe and the Mediterranean region, central Europe, central North America, Central America and Mexico, northeast Brazil, and southern Africa. Elsewhere there is overall low confidence because of insufficient agreement of projections of drought changes (dependent both on model and dryness index). Definitional issues and lack of data preclude higher confidence than medium in observations of drought changes, while these issues plus the inability of models to include all the factors likely to influence droughts preclude stronger confidence than medium in the projections.

\subsubsection{Floods}

A flood is "the overflowing of the normal confines of a stream or other body of water, or the accumulation of water over areas that are not normally submerged (some specific examples are discussed in Case Study 9.2.6). Floods include river (fluvial) floods, flash floods, urban floods, pluvial floods, sewer floods, coastal floods, and glacial lake outburst floods" (see Glossary). The main causes of floods are intense and/or long-lasting precipitation, snow/ice melt, a combination of these causes, dam break (e.g., glacial lakes), reduced conveyance due to ice jams or landslides, or by a local intense storm (Smith and Ward, 1998). Floods are affected by various characteristics of precipitation, such as intensity, duration, amount, timing, and phase (rain or snow). They are also affected by drainage basin conditions such as water levels in the rivers, the presence of snow and ice, soil character and status (frozen or not, soil moisture content and vertical distribution), rate and timing of snow/ice melt, urbanization, and the existence of dikes, dams, and reservoirs (Bates et al., 2008). Along coastal areas, flooding may be associated with storm surge events (Section 3.5.5). A change in the climate physically changes many of the factors affecting floods (e.g., precipitation, snow cover, soil moisture content, sea level, glacial lake conditions, vegetation) and thus may consequently change the characteristics of floods. Engineering developments such as dikes and reservoirs regulate flow, and land use may also affect floods. Therefore the assessment of causes of changes in floods is complex and difficult. The focus in this section is on changes in floods that might be related to changes in climate (i.e., referred to as 'climate-driven'), rather than changes in engineering developments or land use. However, because of partial lack of documentation, these can be difficult to distinguish in the instrumental record.

Literature on the impact of climate change on pluvial floods (e.g., flash floods and urban floods) is scarce, although the changes in heavy precipitation discussed in Section 3.3.2 may imply changes in pluvial floods in some regions. This chapter focuses on the spatial, temporal, and seasonal changes in high flows and peak discharge in rivers related to climate change, which cause changes in fluvial (river) floods. River discharge simulation under a changing climate scenario requires a set of GCM or RCM outputs (e.g., precipitation and surface air temperature) and a hydrological model. A hydrological model may consist of a land surface model of a GCM or RCM and a river routing model. Different hydrological models may yield quantitatively different river discharge, but they may not yield different signs of the trend if the same GCM/ RCM outputs are used. So the ability of models to simulate floods, in particular regarding the signs of the past and future trends, depends on the ability of the GCM or RCM to simulate precipitation changes. The ability of a GCM or RCM to simulate temperature is important for river discharge simulation in snowmelt- and glacier-fed rivers. Downscaling and/or bias-correction are frequently applied to GCM/RCM outputs before hydrological simulations are conducted, which becomes a source of uncertainty. More details on the feasibility and uncertainties in hydrological projections are described later in this section. Coastal floods are discussed in Sections 3.5.3 and 3.5.5. Glacial lake outburst floods are discussed in Section 3.5.6. The impact of floods on human society and ecosystems and related changes are discussed in Chapter 4. Case Study 9.2.6 discusses the management of floods.

Worldwide instrumental records of floods at gauge stations are limited in spatial coverage and in time, and only a limited number of gauge stations have data that span more than 50 years, and even fewer more than 100 years (Rodier and Roche, 1984; see also Section 3.2.1). However, this can be overcome partly or substantially by using pre-instrumental flood data from documentary records (archival reports, in Europe continuous over the last 500 years) (Brázdil et al., 2005), and from geological indicators of paleofloods (sedimentary and biological records over centennial to millennial scales) (Kochel and Baker, 1982). Analysis of these pre-instrumental flood records suggest that (1) flood magnitude and frequency can be sensitive to modest alterations in atmospheric circulation, with greater sensitivity for 'rare' floods (e.g., 50-year flood and higher) than for smaller and more frequent floods (e.g., 2-year floods) (Knox, 2000; Redmond et al., 2002); (2) high interannual and interdecadal variability can be found in flood occurrences both in terms of frequency and magnitude although in most cases, cyclic or clusters of flood occurrence are observed in instrumental (Robson et al., 1998), historical (Vallve and Martin-Vide, 1998; Benito et al., 2003; Llasat et al., 2005), and paleoflood records (Ely et al., 1993; Benito et al., 2008); (3) past flood records may contain analogs of unusual large floods, similar to some recorded recently, sometimes considered to be the largest on record. For example, pre-instrumental flood data show that the 2002 summer flood in the Elbe did not reach the highest flood levels recorded in 1118 and 1845 although it was higher than other disastrous floods of 1432, 1805, etc. (Brázdil et al., 2006). However, the currently available pre-instrumental flood data is also limited, particularly in spatial coverage.

The AR4 and the IPCC Technical Paper VI based on the AR4 concluded that no gauge-based evidence had been found for a climate-driven globally widespread change in the magnitude/frequency of floods during the last decades (Rosenzweig et al., 2007; Bates et al., 2008). However, the AR4 also pointed to possible changes that may imply trends in flood occurrence with climate change. For instance, Trenberth et al. (2007) highlighted a catastrophic flood that occurred along several central 
European rivers in 2002, although neither flood nor mean precipitation trends could be identified in this region; however, there was a trend toward increasing precipitation variability during the last century which itself could imply an enhanced probability of flood occurrence. Kundzewicz et al. (2007) argued that climate change (i.e., observed increase in precipitation intensity and other observed climate changes) might already have had an impact on floods. Regarding the spring peak flows, the AR4 concluded with high confidence that abundant evidence was found for an earlier occurrence in snowmelt- and glacier-fed rivers (Rosenzweig et al., 2007; Bates et al., 2008), though we expressly note here that a change in the timing of peak flows does not necessarily imply nor preclude changes in flood magnitude or frequency in the affected regions.

Although changes in flood magnitude/frequency might be expected in regions where temperature change affects precipitation type (i.e., rain/ snow separation), snowmelt, or ice cover (in particular northern highlatitude and polar regions), widespread evidence of such climate-driven changes in floods is not available. For example, there is no evidence of widespread common trends in the magnitude of floods based on the daily river discharge of 139 Russian gauge stations for the last few to several decades, though a significant shift in spring discharge to earlier dates has been found (Shiklomanov et al., 2007). Lindström and Bergström (2004) noted that it is difficult to conclude that flood levels are increasing from an analysis of runoff trends in Sweden for 1807 to 2002.

In the United States and Canada during the 20th century and in the early 21 st century, there is no compelling evidence for climate-driven changes in the magnitude or frequency of floods (Lins and Slack, 1999; Douglas et al., 2000; McCabe and Wolock, 2002; Cunderlik and Ouarda, 2009; Villarini et al., 2009). There are relatively abundant studies on the changes and trends for rivers in Europe such as rivers in Germany and its neighboring regions (Mudelsee et al., 2003; Tu et al., 2005; Yiou et al., 2006; Petrow and Merz, 2009), in the Swiss Alps (Allamano et al., 2009), in France (Renard et al., 2008), in Spain (Benito et al., 2005), and in the United Kingdom (Robson et al., 1998; Hannaford and Marsh, 2008), but a continental-scale assessment of climate-driven changes in the flood magnitude and frequency for Europe is difficult to provide because geographically organized patterns are not seen in the reported changes.

Available (limited) analyses for Asia suggest the following changes: the annual flood maxima of the lower Yangtze region show an upward trend over the last 40 years (Jiang et al., 2008), the likelihood for extreme floods in the Mekong River has increased during the second half of the 20th century although the probability of an average flood has decreased (Delgado et al., 2009), and both upward and downward trends are identified over the last four decades in four selected river basins of the northwestern Himalaya (Bhutiyani et al., 2008). In the Amazon region in South America, the 2009 flood set record highs in the 106 years of data for the Rio Negro at the Manaus gauge site in July 2009 (Marengo et al., 2011). Recent increases have also been reported in flood frequency in some other river basins in South America (Camilloni and Barros, 2003; Barros et al., 2004). Conway et al. (2009) concluded that robust identification of hydrological change was severely limited by data limitations and other issues for sub-Saharan Africa. Di Baldassarre et al. (2010) found no evidence that the magnitude of African floods has increased during the 20th century. However, such analyses cover only limited parts of the world. Evidence in the scientific literature from the other parts of the world, and for other river basins, appears to be very limited.

Many river systems are not in their natural state anymore, making it difficult to separate changes in the streamflow data that are caused by the changes in climate from those caused by human regulation of the river systems. River engineering and land use may have altered flood probability. Many dams are designed to reduce flooding. Large dams have resulted in large-scale land use change and may have changed the effective rainfall in some regions (Hossain et al., 2009).

The above analysis indicates that research subsequent to the AR4 still does not show clear and widespread evidence of climate-driven observed changes in the magnitude or frequency of floods at the global level based on instrumental records, and there is thus low confidence regarding the magnitude and frequency and even the sign of these changes. The main reason for this lack of confidence is due to limited evidence in many regions, since available instrumental records of floods at gauge stations are limited in space and time, which limits the number of analyses. Moreover, the confounding effects of changes in land use and engineering mentioned above also make the identification of climate-driven trends difficult. There are limited regions with medium evidence, where no ubiquitous change is apparent (low agreement). Pre-instrumental flood data can provide information for longer periods, but current availability of these data is even scarcer particularly in spatial coverage. There is abundant evidence for an earlier occurrence of spring peak flows in snowmelt- and glacier-fed rivers (high confidence), though this feature may not necessarily be linked with changes in the magnitude of spring peak flows in the concerned regions.

The possible causes for changes in floods were discussed in the AR4 and Bates et al. (2008), but cause-and-effect between external forcing and changes in floods was not explicitly assessed. A rare example considered in Rosenzweig et al. (2007) and Bates et al. (2008) was a study by Milly et al. (2002) which, based on monthly river discharge, reported an impact of anthropogenic climate change on changes (mostly increases) in 'large' floods during the 20th century in selected extratropical river basins larger than $20,000 \mathrm{~km}^{2}$, but they did not endorse the study because of the lack of widespread observed evidence of such trends in other studies. More recent literature has detected the influence of anthropogenically induced climate change in variables that affect floods, such as aspects of the hydrological cycle (see Section 3.2.2.2) including mean precipitation (X. Zhang et al., 2007), heavy precipitation (see Section 3.3.2), and snowpack (Barnett et al., 2008), though a direct statistical link between anthropogenic climate change and trends in the magnitude and frequency of floods is still not established. 
In climates where seasonal snow storage and melting play a significant role in annual runoff, the hydrologic regime is affected by changes in temperature. In a warmer world, a smaller portion of precipitation falls as snow (Hirabayashi et al., 2008a) and the melting of winter snow occurs earlier in spring, resulting in a shift in peak river runoff to winter and early spring. This has been observed in the western United States (Regonda et al., 2005; Clow, 2010), in Canada (Zhang et al., 2001), and in other cold regions (Rosenzweig et al., 2007; Shiklomanov et al., 2007), along with an earlier breakup of river ice in Arctic rivers (Smith, 2000; Beltaos and Prowse, 2009). The observed trends toward earlier timing of snowmelt-driven streamflows in the western United States since 1950 are detectably different from natural variability (Barnett et al., 2008; Hidalgo et al., 2009). Thus, observed warming over several decades that is attributable to anthropogenic forcing has likely been linked to earlier spring peak flows in snowmelt- and glacier-fed rivers. It is unclear if observed warming over several decades has affected the magnitude of the snowmelt peak flows, but warming may result either in an increase in spring peak flows where winter snow depth increases (Meehl et al., 2007b) or a decrease in spring peak flows because of decreased snow cover and amounts (Hirabayashi et al., 2008b; Dankers and Feyen, 2009).

There is still a lack of studies identifying an influence of anthropogenic climate change over the past several decades on rain-generated peak streamflow trends because of availability and uncertainty in the observed streamflow data and low signal-to-noise ratio. Evidence has recently emerged that anthropogenic climate change could have increased the risk of rainfall-dominated flood occurrence in some river basins in the United Kingdom in autumn 2000 (Pall et al., 2011). Overall, there is low confidence (due to limited evidence) that anthropogenic climate change has affected the magnitude and frequency of floods, though it has detectably influenced several components of the hydrological cycle, such as precipitation and snowmelt, that may impact flood trends. The assessment of causes behind the changes in floods is inherently complex and difficult.

The number of studies that investigated projected flood changes in rivers especially at a regional or a continental scale was limited when the AR4 was published. Projections of flood changes at the catchment/ river-basin scale were also not abundantly cited in the AR4. Nevertheless, Kundzewicz et al. (2007) and Bates et al. (2008) argued that more frequent heavy precipitation events projected over most regions would affect the risk of rain-generated floods (e.g., flash flooding and urban flooding).

The number of regional- or continental-scale studies of projected changes in floods is still limited. Recently, a few studies for Europe (Lehner et al., 2006; Dankers and Feyen, 2008, 2009) and a study for the globe (Hirabayashi et al., 2008b) have indicated changes in the frequency and/or magnitude of floods in the 21st century at large scale using daily river discharge calculated from RCM or GCM outputs and hydrological models. A notable change is projected to occur in northeastern Europe in the late 21 st century because of a reduction in snow accumulation
(Dankers and Feyen, 2008, 2009; Hirabayashi et al., 2008b), that is, a decrease in the probability of floods, that generally corresponds to lower flood peaks. For other parts of the world, Hirabayashi et al. (2008b) show an increase in the risk of floods in most humid Asian monsoon regions, tropical Africa, and tropical South America with a decrease in the risk of floods in non-negligible areas of the world such as most parts of northern North America.

Projections of flood changes at the catchment/river-basin scale are also not abundant in the scientific literature. Several studies have been undertaken for UK catchments (Cameron, 2006; Kay et al., 2009; Prudhomme and Davies, 2009) and catchments in continental Europe and North America (Graham et al., 2007; Thodsen, 2007; Leander et al., 2008; Raff et al., 2009; van Pelt et al., 2009). However, projections for catchments in other regions such as Asia (Asokan and Dutta, 2008; Dairaku et al., 2008), the Middle East (Fujihara et al., 2008), South America (Nakaegawa and Vergara, 2010; Kitoh et al., 2011), and Africa (Taye et al., 2011) are rare.

Uncertainty is still large in the projected changes in the magnitude and frequency of floods. It has been recently recognized that the choice of GCMs is the largest source of uncertainties in hydrological projections at the catchment/river-basin scale, and that uncertainties from emission scenarios and downscaling methods are also relevant but less important (Graham et al., 2007; Leander et al., 2008; Kay et al., 2009; Prudhomme and Davies, 2009), although, in general, hydrological projections require downscaling and/or bias-correction of GCM outputs (e.g., precipitation and temperature). Also the choice of hydrological models was found to be relevant but less important (Kay et al., 2009; Taye et al., 2011). However, the relative importance of downscaling, bias-correction, and the choice of hydrological models may depend on the selected region/ catchment, the selected downscaling and bias-correction methods, and the selected hydrological models (Wilby et al., 2008). For example, the sign of the above-mentioned flood changes in northeastern Europe is affected by differences in temporal downscaling and bias-correction methods applied in the different studies (Dankers and Feyen, 2009). Chen et al. (2011) demonstrated considerable uncertainty caused by several downscaling methods in a hydrological projection for a snowmelt-dominated Canadian catchment. Downscaling (see Section 3.2.3) and bias-correction are also a major source of uncertainty in raindominated catchments (van Pelt et al., 2009). We also note that biascorrection and statistical downscaling tend to ignore the energy closure of the climate system, which could be a non-negligible source of uncertainty in hydrological projections (Milly and Dunne, 2011).

The number of projections of flood magnitude and frequency changes is still limited at regional and continental scales. Projections at the catchment/river-basin scale are also not abundant in the peer-reviewed scientific literature, especially for regions outside Europe and North America. In addition, considerable uncertainty remains in the projections of flood changes, especially regarding their magnitude and frequency. Therefore, our assessment is that there is low confidence (due to limited evidence) in future changes in flood magnitude and frequency derived 
from river discharge simulations. Nevertheless, as was argued by Kundzewicz et al. (2007) and Bates et al. (2008), physical reasoning suggests that projected increases in heavy rainfall in some catchments or regions would contribute to increases in rain-generated local floods (medium confidence). We note that heavy precipitation may be projected to increase despite a projected decrease of total precipitation depending on the regions considered (Section 3.3.2), and that changes in several variables (e.g., precipitation totals, frequency, and intensity, snow cover and snowmelt, soil moisture) are relevant for changes in floods. Confidence in change in one of these components alone may thus not be sufficient to confidently project changes in flood occurrence. Hence, medium confidence is attached to the above statement based on physical reasoning, although the link between increases in heavy rainfall and increases in local flooding seems apparent. The earlier shifts of spring peak flows in snowmelt- and glacier-fed rivers are robustly projected (Kundzewicz et al., 2007; Bates et al., 2008); so these are assessed as very likely, though this may not necessarily be relevant for flood occurrence. There is low confidence (due to limited evidence) in the projected magnitude of the earlier peak flows in snowmelt- and glacier-fed rivers.

In summary, there is limited to medium evidence available to assess climate-driven observed changes in the magnitude and frequency of floods at a regional scale because the available instrumental records of floods at gauge stations are limited in space and time, and because of confounding effects of changes in land use and engineering. Furthermore, there is low agreement in this evidence, and thus overall low confidence at the global scale regarding even the sign of these changes. There is low confidence (due to limited evidence) that anthropogenic climate change has affected the magnitude or frequency of floods, though it has detectably influenced several components of the hydrological cycle such as precipitation and snowmelt (medium confidence to high confidence), which may impact flood trends. Projected precipitation and temperature changes imply possible changes in floods, although overall there is low confidence in projections of changes in fluvial floods. Confidence is low due to limited evidence and because the causes of regional changes are complex, although there are exceptions to this statement. There is medium confidence (based on physical reasoning) that projected increases in heavy rainfall (Section 3.3.2) would contribute to increases in rain-generated local flooding, in some catchments or regions. Earlier spring peak flows in snowmelt- and glacier-fed rivers are very likely, but there is low confidence in their projected magnitude.

\subsubsection{Extreme Sea Levels}

Transient sea level extremes and extreme coastal high water are caused by severe weather events or tectonic disturbances that cause tsunamis. Since tsunamis are not climate-related, they are not addressed here. The drop in atmospheric pressure and strong winds that accompany severe weather events such as tropical or extratropical cyclones (Sections 3.4.4 and 3.4.5) can produce storm surges at the coast, which may be further elevated by wave setup caused by an onshore flux of momentum due to wave breaking in the surf zone. Various metrics are used to characterize extreme sea levels including storm-related highest values, annual maxima, or percentiles. Extreme sea levels may change in the future as a result of both changes in atmospheric storminess and mean sea level rise. However, neither contribution will be spatially uniform across the globe. For severe storm events such as tropical and extratropical cyclones, changes may occur in the frequency, intensity, or genesis regions of severe storms and such changes may vary between ocean basins (see Sections 3.4.4 and 3.4.5). Along some coastlines, land subsidence due to glacial isostatic adjustment (e.g., Lambeck et al., 2010 ) is causing a relative fall in sea levels. Variations in the rate of sea level rise can be large relative to mean sea level (Yin et al., 2010) and will occur as a result of variations in wind change (e.g., Timmermann et al., 2010), changes in atmospheric pressure and oceanic circulation (e.g., Tsimplis et al., 2008), and associated differences in water density and rates of thermal expansion (e.g., Bindoff et al., 2007; Church et al., 2010; Yin et al., 2010). In addition, if rapid melting of ice sheets occurs it would lead to non-uniform rates of sea level rise across the globe due to adjustments in the Earth's gravitational field (e.g., Mitrovica et al., 2010). On some coastlines, higher mean sea levels may alter the astronomical tidal range and the evolution of storm surges, and increase the wave height in the surf zones. As well as gradual increases in mean sea level that contribute to extreme impacts from transient extreme sea levels, rapid changes in sea level arising from, for example collapse of ice shelves could be considered to be an extreme event with the potential to contribute to extreme impacts in the future. However, knowledge about the likelihood of such changes occurring is limited and so does not allow an assessment at this time.

Mean sea level has varied considerably over glacial time scales as the extent of ice caps and glaciers have fluctuated with global temperatures. Sea levels have risen around 120 to $130 \mathrm{~m}$ since the last glacial maximum 19 to $23 \mathrm{ka}$ before present to around 7,000 years ago, and reached a level close to present at least 6,000 years ago (Lambeck et al., 2010). As well as the influence on sea level extremes caused by rapidly changing coastal bathymetries (Clarke and Rendell, 2009) and large-scale circulation patterns (Wanner et al., 2008), there is some evidence that changes in the behavior of severe tropical cyclones has changed on centennial time scales, which points to non-stationarity in extreme sea level events (Nott et al., 2009). Woodworth et al. (2011) use tide gauge records dating back to the 18th century, and salt marsh data, to show that sea level rise has accelerated over this time frame.

The AR4 reported that there was high confidence that the rate of observed sea level rise increased from the 19th to the 20th century (Bindoff et al., 2007). It also reported that the global mean sea level rose at an average rate of 1.7 (1.2 to 2.2$) \mathrm{mm} \mathrm{yr}^{-1}$ over the 20th century, 1.8 (1.3 to 2.3) $\mathrm{mm} \mathrm{yr}^{-1}$ over 1961 to 2003 , and at a rate of 3.1 (2.4 to 3.8$) \mathrm{mm} \mathrm{yr}^{-1}$ over 1993 to 2003. With updated satellite data to 2010, Church and White (2011) show that satellite-measured sea levels continue to rise at 
a rate close to that of the upper range of the AR4 projections. Whether the faster rate of increase during the latter period reflects decadal variability or an increase in the longer-term trend is not clear. However, there is evidence that the contribution to sea level due to mass loss from Greenland and Antarctica is accelerating (Velicogna, 2009; Rignot et al., 2011; Sørensen et al., 2011). The AR4 also reported that the rise in mean sea level and variations in regional climate led to a likely increase in the trend of extreme high water worldwide in the late 20th century (Bindoff et al., 2007), it was very likely that humans contributed to sea level rise during the latter half of the 20th century (Hegerl et al., 2007), and therefore that it was more likely than not that humans contributed to the trend in extreme high sea levels (IPCC, 2007a). Since the AR4, Menendez and Woodworth (2010), using data from 258 tide gauges across the globe, have confirmed the earlier conclusions of Woodworth and Blackman (2004) that there was an increasing trend in extreme sea levels globally, more pronounced since the 1970s, and that this trend was consistent with trends in mean sea level (see also Lowe et al., 2010). Additional studies at particular locations support this finding (e.g., Marcos et al., 2009; Haigh et al., 2010).

Various studies also highlight the additional influence of climate variability on extreme sea level trends. Menendez and Woodworth (2010) report that ENSO (see Section 3.4.2) has a large influence on interannual variations in extreme sea levels in the Pacific Ocean and the monsoon regions based on sea level records since the 1970s. In southern Europe, Marcos et al. (2009) report that changes in extremes are also significantly negatively correlated with the NAO (see Section 3.4.3). Ullmann et al. (2007) concluded that maximum annual sea levels in the Camargue had risen twice as fast as mean sea level during the 20th century due to an increase in southerly winds associated with a general rise in sea level pressure over central Europe (Ullmann et al., 2008). Sea level trends from two tide gauges on the north coast of British Columbia from 1939 to 2003 were twice that of mean sea level rise, the additional contribution being due to the strong positive phase of the PDO (see Section 3.4.3), which has lasted since the mid-1970s (Abeysirigunawardena and Walker, 2008). Cayan et al. (2008) reported an increase of 20-fold at San Francisco since 1915 and 30-fold at La Jolla since 1933 in the frequency of exceedance of the 99.99th percentile sea level. They also noted that positive sea level anomalies of 10 to $20 \mathrm{~cm}$ that often persisted for several months during El Niño events produced an increase in storm surge peaks over this time. The spatial extent of these oscillations and their influence on extreme sea levels across the Pacific has been discussed by Merrifield et al. (2007). Church et al. (2006a) examined changes in extreme sea levels before and after 1950 in two tide gauge records of approximately 100 years on the east and west coasts of Australia, respectively. At both locations a stronger positive trend was found in the sea level exceeded by $0.01 \%$ of the observations than the median sea level, suggesting that in addition to mean sea level rise, other modes of variability or climate change are contributing to the extremes. At Mar del Plata, Argentina, Fiore et al. (2009) noted an increase in the number and duration of positive storm surges in the decade 1996 to 2005 compared to previous decades, which may be due to a combination of mean sea level rise and changes in wind climatology resulting from a southward shift in the South Atlantic high.

Thus, studies since the AR4 conclude that trends in extreme sea level are generally consistent with changes in mean sea level (e.g., Marcos et al., 2009; Haigh et al., 2010; Menendez and Woodworth, 2010) although some studies note that the trends in extremes are larger than the observed trend in mean sea levels (e.g., Church et al., 2006a; Ullmann et al., 2007; Abeysirigunawardena and Walker, 2008) and may be influenced by modes of climate variability, such as the PDO on the Canadian west coast (e.g., Abeysirigunawardena and Walker, 2008). These studies are consistent with the conclusions from the AR4 that increases in extremes are related to trends in mean sea level and modes of variability in the regional climate.

The AR4 (Meehl et al., 2007b) projected sea level rise for 2090-2099 relative to 1980-1999 due to ocean thermal expansion, glaciers and ice caps, and modeled ice sheet contributions of 18 to $59 \mathrm{~cm}$, which incorporates a $90 \%$ uncertainty range across all scenarios. An additional contribution to the sea level rise projections was taken into account for a possible rapid dynamic response of the Greenland and West Antarctic ice sheets, which could result in an accelerating contribution to sea level rise. This was estimated to be 10 to $20 \mathrm{~cm}$ of sea level rise by 2090-2099 using a simple linear relationship with projected temperature. Because of insufficient understanding of the dynamic response of ice sheets, Meehl et al. (2007b) also noted that a larger contribution could not be ruled out.

Several studies since the AR4 have developed statistical models that relate 20th-century (e.g., Rahmstorf, 2007; Horton et al., 2008) or longer (e.g.,Vermeer and Rahmstorf, 2009; Grinsted et al., 2010) temperature and sea level rise to extrapolate future global mean sea level. These alternative approaches yield projections of sea level rise under a range of SRES scenarios by 2100 of 0.47 to $1.00 \mathrm{~m}$ (B1 to $\mathrm{A} 2$ scenarios; Horton et al., 2008), 0.50 to $1.40 \mathrm{~m}$ (B1 to A1FI scenarios; Rahmstorf, 2007), 0.75 to $1.90 \mathrm{~m}$ (B1 to A1Fl scenarios; Vermeer and Rahmstorf, 2009), and 0.90 to $1.30 \mathrm{~m}$ (A1B scenario only; Grinsted et al., 2010). However, future rates of sea level rise may be less closely associated with global mean temperature if ice sheet dynamics play a larger role in the future (Cazenave and Llovel, 2010). Furthermore, Church et al. (2011) note that these models may overestimate future sea levels because non-climate related contributions to trends over the observational period such as groundwater depletion may not have been removed, and non-linear effects such as the reduction in glacier area as glaciers contract and the reduction in the efficiency of ocean heat uptake with global warming in the future are not accounted for. Pfeffer et al. (2008), using a dynamical model of glaciers, found that sea level rise of more than $2 \mathrm{~m}$ by 2100 is physically implausible. An estimate of $0.8 \mathrm{~m}$ by 2100 that included increased ice dynamics was considered most plausible.

New studies, whose focus is on quantifying the effect of storminess changes on storm surge, have been carried out over northern Europe since the AR4. Debernard and Roed (2008) used hydrodynamic models 
to investigate storm surge changes over Europe in four regionally downscaled GCMs including two runs with $B 2$, one with $A 2$, and one with an $\mathrm{A} 1 \mathrm{~B}$ emission scenario. Despite large inter-model differences, statistically significant changes between 1961-1990 and 2071-2100 consisted of decreases in the 99th percentile surge heights south of Iceland, and an 8 to $10 \%$ increase along the coastlines of the eastern North Sea and the northwest British Isles, which occurred mainly in the winter season. Wang et al. (2008) projected a significant increase in wintertime storm surges around Ireland except the south Irish coast over 2031-2060 relative to 1961-1990 using a downscaled GCM under an A1B scenario. Sterl et al. (2009) joined the output from an ensemble of 17 GCM (CMIP3) simulations using the A1B emissions scenario over the model periods $1950-2000$ and 2050-2100 into a single longer time series to estimate 10,000-year return values of surge heights along the Dutch coastline. No statistically significant change in this value was projected for the 21st century because projected wind speed changes were not associated with the surge-generating northerlies but rather non-surge generating south-westerlies.

Other studies have undertaken a sensitivity approach to compare the relative impact on extreme sea levels of severe weather changes and mean sea level rise. Over southeastern Australia, McInnes et al. (2009b) found that a $10 \%$ increase in wind speeds, consistent with the upper end of the range under an A1FI scenario from a multi-model ensemble for 2070 together with an A1FI sea level rise scenario, would produce extreme sea levels that were 12 to $15 \%$ higher than those including just the A1FI sea level rise projection alone. Brown et al. (2010) also investigated the relative impact of sea level rise and wind speed change on an extreme storm surge in the eastern Irish Sea. Both studies concluded that sea level rise rather than meteorological changes has the greater potential to increase extreme sea levels in these locations in the future.

The degree to which climate models (GCM or RCM) have sufficient resolution and/or internal physics to realistically capture the meteorological forcing responsible for storm surges is regionally dependent. For example current GCMs are unable to realistically represent tropical cyclones (see Section 3.4.4). This has led to the use of alternative approaches for investigating the impact of climate change on storm surges in tropical locations whereby large numbers of cyclones are generated using statistical models that govern the cyclones' characteristics over the observed period (e.g., Mclnnes et al., 2003). These models are then perturbed to represent projected future cyclone characteristics and used to force a hydrodynamic model. Recent studies on the tropical east coast of Australia reported in Harper et al. (2009) that employ these approaches show a relatively small impact of a $10 \%$ increase in tropical cyclone intensity on the 1-in-100 year storm tide (the combined sea level due to the storm surge and tide), and mean sea level rise being found to produce the larger contribution to changes in future 1-in-100 year sea level extremes. However, one study that has incorporated scenarios of sea level rise in the hydrodynamic modeling of hurricane-induced sea level extremes on the Louisiana coast found that increased coastal water depths had a large impact on surge propagation over land, increasing storm surge heights by two to three times the sea level rise scenario, particularly in wetland-fronted areas (J.M. Smith et al., 2010).

To summarize, post-AR4 studies provide additional evidence that trends in extreme coastal high water across the globe reflect the increases in mean sea level, suggesting that mean sea level rise rather than changes in storminess are largely contributing to this increase (although data are sparse in many regions and this lowers the confidence in this assessment). It is therefore considered likely that sea level rise has led to a change in extreme coastal high water levels. It is likely that there has been an anthropogenic influence on increasing extreme coastal high water levels via mean sea level contributions. While changes in storminess may contribute to changes in sea level extremes, the limited geographical coverage of studies to date and the uncertainties associated with storminess changes overall (Sections 3.4.4 and 3.4.5) mean that a general assessment of the effects of storminess changes on storm surge is not possible at this time. On the basis of studies of observed trends in extreme coastal high water levels it is very likely that mean sea level rise will contribute to upward trends in the future.

\subsubsection{Waves}

Severe waves threaten the safety of coastal inhabitants and those involved in maritime activities and can damage and destroy coastal and marine infrastructure. Waves play a significant role in shaping a coastline by transporting energy from remote areas of the ocean to the coast. Energy dissipation via wave breaking contributes to beach erosion, longshore currents, and elevated coastal sea levels through wave set-up and wave run-up. Wave properties that influence these processes include wave height, the wave energy directional spectrum, and period. Studies of past and future changes in wave climate to date have tended to focus on wave height parameters such as 'Significant Wave Height' (SWH, the average height from trough to crest of the highest one-third of waves) and metrics of extreme waves, such as high percentiles or wave heights above particular thresholds, although one study (Dodet et al., 2010) also examines trends in mean wave direction and peak wave period. It should also be noted that waves may become an increasingly important factor along coastlines experiencing a decline in coastal protection afforded by sea ice (see Sections 3.5.5 and 3.5.7).

Wave climates have changed over paleoclimatic time scales. Wave modeling using paleobathymetries over the past 12,000 years indicates an increase in peak annual SWH of around $40 \%$ due to the increase in mean sea level, which redefines the location of the coastline, and hence progressively extends the fetch length in most of the shelf sea regions (Neill et al., 2009). Major circulation changes that result in changes in storminess and wind climate (see Section 3.3.3) have also affected wave climates. Evidence of enhanced storminess determined from sand drift and dune building along the western European coast indicates that enhanced storminess occurred over the period of the Little Ice Age 
(1570-1900) and the mid Holocene ( 8,200 years before present; Clarke and Rendell, 2009).

The AR4 reported statistically significant positive trends in SWH over the period 1950 to 2002 over most of the mid-latitudinal North Atlantic and North Pacific, as well as in the western subtropical South Atlantic, the eastern equatorial Indian Ocean and the East China and South China Sea, and declining trends around Australia, and parts of the Philippine, Coral, and Tasman Seas (Trenberth et al., 2007), based on voluntary observing ship data (e.g., Gulev and Grigorieva, 2004). Several studies that address trends in extreme wave conditions have been completed since the AR4 and the new studies generally provide more evidence for the previously reported positive trends in SWH and extreme waves in the north Atlantic and north Pacific. Global trends in 99th-percentile satellite-measured wave heights show a mostly significant positive trend of between 0.5 and $1.0 \%$ per year in the mid-latitude oceans but less clear trends over the tropical oceans from 1985 to 2008 (Young et al., 2011). X.L. Wang et al. (2009b) found that SWH increased in the boreal winter over the past half century in the high latitudes of the Northern Hemisphere (especially the northeast Atlantic), and decreased in more southerly northern latitudes based on the European Centre for Medium Range Weather Forecasts 40-year reanalysis (ERA-40). They also found that storminess around the 1880s was of similar magnitude to that in the 1990s. This is also found using the same data set by Le Cozannet et al. (2011), who relate the change in waves to the NAO pattern that is moderated by an east Atlantic pattern of climate variability during winter. A wave hindcast over the north-eastern Atlantic Ocean over the period 1953 to 2009 revealed a significant positive trend in SWH, as well as a counterclockwise shift in mean direction in the north and a slight but not significant increase in peak wave period in the northeast. In the south, no trend was found for SWH or wave period while a clockwise trend in mean direction was found (Dodet et al., 2010). In a regional North Sea hindcast, Weisse and Günther (2007) found a positive trend in 99th-percentile wave height from 1958 to the early 1990s followed by a declining trend to 2002 over the southern North Sea, except on the UK North Sea coast where negative trends occurred over much of the hindcast period.

On the North American Atlantic coast, Komar and Allan (2008) found a statistically significant trend of $0.059 \mathrm{~m} \mathrm{yr}^{-1}$ in waves exceeding $3 \mathrm{~m}$ during the summer months over 30 years since the mid-1970s at Charleston, South Carolina, with weaker but statistically significant trends at wave buoys further north. These trends were associated with an increase in intensity and frequency of hurricanes over this period (see Section 3.4.4). In contrast, winter waves, generated by extratropical storms, were not found to have experienced a statistically significant change. In the eastern North Pacific, SWH is strongly correlated with El Niño (Section 3.4.2). However positive trends were also found in SWH and extreme wave height from the mid-1970s to 2006 in wave buoy data (Allan and Komar, 2006), for excesses of the 98th percentile SWH over 1985 to 2007 (Menendez et al., 2008) along the US west coast, and in hindcast SWH over 1948 to 1998 in the Southern Californian Bight (Adams et al., 2008). Positive though not statistically significant trends in annual mean SWH were found over south-eastern South America for in situ wave data over the 1996-2006 period and in satellite wave data over 1993 to 2001, while simulated wave fields using reanalysis wind forcing over the period 1971 to 2005 produced statistically significant trends in SWH (Dragani et al., 2010). Trends at particular locations may be also influenced by local factors. For example, Suursaar and Kullas (2009) reported a slight decreasing trend in mean SWHs from 1966 to 2006 in the Gulf of Riga within the Baltic Sea, while the frequency and intensity of high wave events (i.e., the difference between the maximum and 99th-percentile wave height) showed rising trends. These changes were associated with a decrease in local average wind speed, but an intensification of westerly winds and storm events occurring further to the west.

In the Southern Ocean, SWH derived from satellite observations was found to be strongly positively correlated with the SAM, particularly from March to August (Hemer et al., 2010). However, the analysis of reliable long-term trends in the Southern Hemisphere remains challenging due to limited in situ data and problems of temporal homogeneity in reanalysis products (Wang et al., 2006). For example, Hemer et al. (2010) also found that trends in SWH derived from satellite data over 1998-2000 relative to 1993-1996 were positive only over the Southern Ocean south of $45^{\circ} \mathrm{S}$ whereas trends were positive across most of the Southern Hemisphere in the Corrected ERA-40 reanalysis (C-ERA-40; Hemer, 2010). Hemer (2010) found that the frequency of wave events exceeding the 98th percentile over the period 1985 to 2002 using data from a wave buoy situated on the west coast of Tasmania showed no statistically significant trend whereas a strong positive trend was found in equivalent fields of C-ERA-40 data.

New studies have demonstrated strong links between wave climate and natural modes of climate variability (Section 3.4.3). For example, along the US west coast and the western North Pacific, SWH was found to be strongly correlated with El Niño (Allan and Komar, 2006; Sasaki and Toshiyuki, 2007) and, in the Southern Ocean, SWH was positivity correlated with the SAM (Hemer et al., 2010). On the US east coast, positive trends in summer SWH were linked to increasing numbers of hurricanes (Komar and Allan, 2008). In the northeast Atlantic, trends in SWH exhibited significant positive (negative) correlations with the NAO in the north (south) and more generally, trends in SWH, mean wave direction, and peak wave period over the period 1953 to 2009 were related to the increase in the NAO index over this time (Dodet et al., 2010). One study (X.L. Wang et al., 2009a) reported a link between external forcing (i.e., anthropogenic forcing due to greenhouse gases and aerosols, and natural forcing due to solar and volcanic forcing) and an increase in SWH in the boreal winter in the high latitudes of the Northern Hemisphere (especially the northeast North Atlantic), and a decrease in more southerly northern latitudes over the past half century.

The AR4 projected an increase in extreme wave height in many regions of the mid-latitude oceans as a result of projected increases in wind speeds associated with more intense mid-latitude storms in these regions in a future warmer climate (Meehl et al., 2007b). At the regional scale, 
increases in wave height were projected for most mid-latitude areas analyzed, including the North Atlantic, North Pacific, and Southern Ocean (Christensen et al., 2007) but with low confidence due to low confidence in projected changes in mid-latitude storm tracks and intensities (see Section 3.4.5). Several studies since then have developed wave climate projections that provide stronger evidence for future wave climate change. Global-scale projections of SWH were developed by Mori et al. (2010), using a $1.25^{\circ}$ resolution wave model forced with projected winds from a 20-km global GCM, in which ensemble-averaged SST changes from the CMIP3 models provided the climate forcing. The spatial pattern of projected SWH change between 1979-2004 and 2075-2100 reflects the changes in the forcing winds, which are generally similar to the mean wind speed changes shown in Figure 3-8. Extreme waves (measured by a spatial and temporal average of the top 10 values over the 25 -year period) were projected to exhibit large increases in the northern Pacific, particularly close to Japan due to an increase in strong tropical cyclones and also the Indian Ocean despite decreases in SWH.

A number of regional studies have also been completed since the AR4 in which forcing conditions were obtained for a few selected emission scenarios (typically B2 and A2, representing low-high ranges) from GCMs or RCMs. These studies provide additional evidence for positive projected trends in SWH and extreme waves along the western European coast (e.g., Debernard and Roed, 2008; Grabemann and Weisse, 2008) and the UK coast (Leake et al., 2007), declines in extreme wave height in the Mediterranean sea (Lionello et al., 2008) and the southeast coast of Australia (Hemer et al., 2010), and little change along the Portuguese coast (Andrade et al., 2007). However, considerable variation in projections can arise from the different climate models and scenarios used to force wave models, which lowers the confidence in the projections. For example, along the European North Sea coast, 99th-percentile wave height over the late 21st century relative to the late 20th century is projected to increase by 6 to $8 \%$ by Debernard and Roed (2008) based on wave model simulations with forcing from several GCMs under $A 2, B 2$, and A1B greenhouse gas scenarios, whereas they are projected to increase by up to $18 \%$ by Grabemann and Weisse (2008), who downscaled two GCMs under A2 and B2 emission scenarios. In one region, opposite trends in extreme waves were projected. Grabemann and Weisse (2008) project negative trends in 99th-percentile wave height along the UK North Sea coast, whereas Leake et al. (2007) downscaled the same GCM for the same emission scenarios, using a different RCM, and found positive changes in high percentile wave heights offshore of the East Anglia coastline. A wave projection study by Hemer et al. (2010) concluded that uncertainties arising from the method by which climate model winds were applied to wave model simulations (e.g., by applying bias-correction to winds or perturbing current climate winds with wind changes derived from climate models) made a larger contribution to the spread of RCM projections than the forcing from different GCMs or emission scenarios.

In summary, although post-AR4 studies are few and their regional coverage is limited, their findings generally support the evidence from earlier studies of wave climate trends. Most studies find a link between variations in waves (both SWH and extremes) and internal climate variability. There is low confidence that there has been an anthropogenic influence on extreme wave heights (because of insufficient literature). Despite the existence of downscaling studies for some regions such as the eastern North Sea, there is overall low confidence in wave height projections because of the small number of studies, the lack of consistency of the wind projections between models, and limitations in their ability to simulate extreme winds. However, the strong linkages between wave height and winds and storminess means that it is likely that future negative or positive changes in SWH will reflect future changes in these parameters.

\subsubsection{Coastal Impacts}

Severe coastal hazards such as erosion and inundation are important in the context of disaster risk management and may be affected by climate change through rising sea levels and changes in extreme events. Increasing sea levels will also increase the potential for saltwater intrusion into coastal aquifers. Coastal inundation occurs during periods of extreme sea levels due to storm surges and high waves, particularly when combined with high tides. Although tropical and extratropical cyclones (Sections 3.4.4 and 3.4.5) are the most common causes of sea level extremes, other weather events that cause persistent winds such as anticyclones and fronts can also influence coastal sea levels (Green et al., 2009; McInnes et al., 2009b). In many parts of the world, sea levels are influenced by modes of large scale variability such as ENSO (Section 3.4.2). In the western equatorial Pacific, sea levels can fluctuate up to half a meter between ENSO phases (Church et al., 2006b) and in combination with extremes of the tidal cycle, can cause extensive inundation in lowlying atoll nations even in the absence of extreme weather events (Lowe et al., 2010).

Shoreline position can change from the combined effects of various factors such as:

1) Rising mean sea levels, which cause landward recession of coastlines made up of erodible materials (e.g., Ranasinghe and Stive, 2009)

2) Changes in coastal height due to isostatic rebound (Blewitt et al., 2010; Mitrovica et al., 2010), or sediment compaction from the removal of oil, gas, and water (Syvitski et al., 2009)

3) Changes in the frequency or severity of transient storm erosion events (K.Q. Zhang et al., 2004)

4) Changes in sediment supply to the coast (Stive et al., 2003; Nicholls et al., 2007; Tamura et al., 2010)

5) Changes in wave speed due to sea level rise, which alters wave refraction, or in wave direction, which can cause realignment of shorelines (Ranasinghe et al., 2004; Bryan et al., 2008; Tamura et al., 2010)

6) The loss of natural protective structures such as coral reefs (e.g., Sheppard et al., 2005; Gravelle and Mimura, 2008) due to increased ocean temperatures (Hoegh-Guldberg, 1999) and ocean acidification (Bongaerts et al., 2010) or the reduction in permafrost 
or sea ice in mid- and high latitudes, which exposes soft shores to the effects of waves and severe storms (see Section 3.5.7; Manson and Solomon, 2007).

For example, permafrost degradation and sea ice retreat may contribute to coastal erosion in Arctic regions (see Section 3.5.7).

The susceptibility of coastal regions to erosion and inundation is related to various physical (e.g., shoreline slope), and geomorphological and ecosystem attributes, and therefore may be inferred to some extent from broad coastal characterizations. These include the presence of beaches, rocky shorelines, or coasts with cliffs; deltas; back-barrier environments such as estuaries and lagoons; the presence of mangroves, salt marshes, or sea grasses; and shorelines flanked by coral reefs (e.g., Nicholls et al., 2007) or by permafrost or seasonal sea ice, each of which are characterized by different vulnerability to climate change-driven hazards. For example, deltas are low-lying and hence generally prone to inundation, while beaches are comprised of loose particles and therefore erodible. However, the degree to which these systems are impacted by erosion and inundation will also be influenced by other factors affecting disaster responses. For example, reduced protection from high waves during severe storms could occur as a result of depleted mangrove forests or the degradation of coral reefs (e.g., Gravelle and Mimura, 2008), or loss of sea ice or permafrost (e.g., Manson and Solomon, 2007); there may be a loss of ecosystem services brought about by saltwater contamination of already limited freshwater reserves due to rising sea levels and these will amplify the risks brought about by climate change (McGranahan et al., 2007), and also reduce the resilience of coastal settlements to disasters. Dynamical processes such as vertical land movement also contribute to inundation potential (Haigh et al., 2009). Coastal regions may be rising or falling due to post-glacial rebound or slumping due to aquifer drawdown (Syvitski et al., 2009). Multiple contributions to coastal flooding such as heavy rainfall and flooding in coastal catchments that coincide with elevated sea levels may also be important. Ecosystems such as coral reefs also play an important role in providing material on which atolls are formed. Large-scale oceanic changes that are particularly relevant to both coral reefs and small island countries are discussed in Box 3-4.

As discussed in Section 3.5.3, mean sea level has risen by 120 to $130 \mathrm{~m}$ since the end of the last glacial maximum (Jansen et al., 2007), and this has had a profound effect on coastline position around the world. Coastlines have also evolved over this time frame due to changes in the action of the ocean on the coast through changes in wave climate (Neill et al., 2009) and tides (Gehrels et al., 1995), which arise from the changing geometries of coastlines over glacial time scales and changes in storminess (e.g., Clarke and Rendell, 2009).

The AR4 (Nicholls et al., 2007) reported that coasts are experiencing the adverse consequences of impacts such as increased coastal inundation, erosion, and ecosystem losses. However, attributing these changes to sea level rise is difficult due to the multiple drivers of change over the 20th century (R.J. Nicholls, 2010) and the scarcity and fragmentary nature of data sets that contribute to the problem of identifying and attributing changes (e.g., Defeo et al., 2009). Since the AR4 there have been several new studies that examine coastline changes. In the Caribbean, the beach profiles at 200 sites across 113 beaches and eight islands were monitored on a three-monthly basis from 1985 to 2000, with most beaches found to be eroding and faster rates of erosion generally found on islands that had been impacted by a higher number of hurricanes (Cambers, 2009). However, the relative importance of anthropogenic factors, climate variability, and climate change on the eroding trends could not be separated quantitatively. In Australia, Church et al. (2008) report that despite the positive trend in sea levels during the 20th century, beaches have generally been free of chronic coastal erosion, and where it has been observed it has not been possible to unambiguously attribute it to sea level rise in the presence of other anthropogenic activities. Webb and Kench (2010) argue that the commonly held view of atoll nations being vulnerable to erosion must be reconsidered in the context of physical adjustments to the entire island shoreline, because erosion of some sectors may be balanced by progradation on other sectors. In their survey of 27 atoll islands across three central Pacific Nations (Tuvalu, Kiribati, and Federated States of Micronesia) over a 19- to 61-year period using photography and satellite imagery, they found that $43 \%$ of islands remained stable and $43 \%$ increased in area, with largest rates of increase in island area ranging from 0.1 to 5.6 ha per decade. Only $14 \%$ of islands studied exhibited a net reduction in area. On islands exhibiting either no net change or an increase in area, a larger redistribution of land area was evident in $65 \%$ of cases, consisting of mainly a shoreline recession on the ocean side and an elongation of the island or progradation of the shoreline on the lagoon side. Human settlements were present on 7 of the 27 atolls surveyed and the majority of those exhibited net accretion due in part to coastal protection works. For a coral reef island at the northern end of the Great Barrier Reef, Australia, Dawson and Smithers (2010) report a $6 \%$ increase in area and $4 \%$ increase in volume between 1967 and 2007 but with a net retreat on the east-southeast shoreline and advance on the western side. Chust et al. (2009) evaluated the relative contribution of local anthropogenic (non-climate change related) and sea level rise impacts on the coastal morphology and habitats in the Basque coast, northern Spain, for the period 1954 to 2004. They found that the impact from local anthropogenic influences was about an order of magnitude greater than that due to sea level rise over this period. Increased rates of coastal erosion have also been observed since 1935 in Canada's Gulf of St. Lawrence (Forbes et al., 2004).

The AR4 stated with very high confidence that the impact of climate change on coasts is exacerbated by increased pressures on the physical environment arising from human settlements in the coastal zone (Nicholls et al., 2007). The small number of studies that have been completed since the AR4 have been either unable to attribute coastline changes to specific causes in a quantitative way or else find strong evidence for non-climatic causes that are natural and/or anthropogenic.

The AR4 reported with very high confidence that coasts will be exposed to increasing impacts, including coastal erosion, over coming decades due to climate change and sea level rise, both of which will be 


\section{Box 3-4 | Small Island States}

Small island states represent a distinct category of locations owing to their small size and highly maritime climates, which means that their concerns and information needs in relation to future climate change differ in many ways from those of the larger continental regions that are addressed in this chapter. Their small land area and often low elevation makes them particularly vulnerable to rising sea levels and impacts such as inundation, shoreline change, and saltwater intrusion into underground aquifers (Mimura, 1999). Their maritime environments lead to an additional emphasis on oceanic information to understand the impacts of climate change (see Case Study 9.2.9). Particular challenges exist for the assessment of past changes in climate given the sparse regional and temporal coverage of terrestrial-based observation networks and the limited in situ ocean observing network, although observations have improved somewhat in recent decades with the advent of satellite-based observations of meteorological and oceanic variables. However, the short length of these records hampers the investigation of long-term trends in the region. The resolution of GCMs is insufficient to represent small islands and few studies have been undertaken to provide projections for small islands using RCMs (Campbell et al., 2011). In regions such as the Pacific Ocean, large-scale climate features such as the South Pacific Convergence Zone ENSO (Section 3.4.2) have a substantial influence on the pattern and timing of precipitation, yet these features and processes are often poorly represented in GCMs (Collins et al., 2010). The purpose of this box is to present available information on observed trends and climate change projections that are not covered in the other sections of this chapter as well as discuss key aspects of the climate system that are particularly relevant for small islands. The very likely contribution of mean sea level rise to increased extreme sea levels (see Section 3.5.3), coupled with the likely increase in tropical cyclone maximum wind speed (see Section 3.4.4), is a specific issue for tropical small island states.

Although the underlying data sources are limited, some data for the Indian Ocean, South Pacific (Fiji), and Caribbean were available in the studies of Alexander et al. (2006) and Caesar et al. (2011). Problems of data availability and homogeneity for the Caribbean are discussed by T.S. Stephenson et al. (2008). Based on standard extremes indices, positive trends in warm days and warm nights and negative trends in cold days and cold nights ${ }^{2}$ have occurred across the Indian Ocean and South Pacific region for the period 1971 to 2005 (Caesar et al., 2011) and the Caribbean for the period 1951 to 2003 (based on data from Alexander et al., 2006). Based on the same data sources, trends in average total wet-day precipitation were positive and statistically significant over the Indian Ocean region, negative over the South Pacific region, and weakly negative over the Caribbean. Trends in heavy and very heavy precipitation were positive over the Indian Ocean, negative over the South Pacific region, and close to zero over the Caribbean. We have low confidence in temperature trends over the Indian Ocean and South Pacific region due to the shorter record over which trends were assessed, whereas for the Caribbean, we have medium confidence in the temperature trends due to the longer records available for assessment. Because of the spatial heterogeneity exhibited in precipitation trends in general, there is insufficient evidence to assess observed rainfall trends. For the Caribbean, temperatures are projected to increase across the region by 1 to $4^{\circ} \mathrm{C}$ over $2071-2100$ relative to 1961-1990 under the A2 and B2 scenarios and rainfall is mainly projected to decrease by 25 to $50 \%$ except in the north (Campbell et al., 2011). Based on this study and the evidence for projected temperature increases reported for other regions (see Table 3-3) we have medium confidence in the projected temperature increases for the Caribbean. However, due to the range of processes that contribute to rainfall change, some of which are poorly resolved by GCMs, there is insufficient evidence to assess projected rainfall changes on these small islands.

Given the low elevation of many small islands, sea level extremes are of particular relevance. Sea level extremes are strongly influenced by tidal extremes (Chowdhury et al., 2007; Merrifield et al., 2007). When the tide behavior is mostly semi-diurnal (two high and low tides per day), there will be a clustering of high spring tides around the time of the equinoxes whereas when the tide behavior is diurnal (one high and low tide per day), the clustering of high spring tides will occur around the time of the solstices. In addition, ENSO has a strong influence such that sea levels and their extremes are positively (negatively) correlated with the SOI in the tropical Pacific west (east) of $180^{\circ}$ (Church et al., 2006b; Menendez et al., 2010). Tides and ENSO have contributed to the more frequent occurrence of sea level extremes and associated flooding experienced at some Pacific Islands such as Tuvalu in recent years, and make the task of determining the relative roles of these natural effects and mean sea level rise difficult (Lowe et al., 2010). Furthermore, the steep shelf margins that surround many islands and atolls in the Pacific support larger wave-induced contributions to sea level anomalies. Unfortunately, wave observations (including wave direction) that would facilitate more comprehensive studies of tide, surge, and wave extremes in the region are sparse, including those that are co-located with tide gauges (Lowe et al., 2010).

\footnotetext{
2 Termed "cool days" and "cool nights" in that study.
} 
Coral reefs are a feature of many small islands and healthy reef systems mitigate against erosion and inundation by not only providing a buffer zone for the shoreline during extreme surge and wave events but also providing a source of carbonate sand and gravel, which are delivered to the shores by storms and swell to maintain the atoll (Woodroffe, 2008; Webb and Kench, 2010). Anthropogenic oceanic changes may indirectly contribute to extreme impacts for coral atolls by affecting the health of the surrounding reef system. Such changes include: (1) warming of the surface ocean, which slows or prevents growth in temperature-sensitive species and causes more frequent coral bleaching events (e.g., Hoegh-Guldberg, 1999; see also Chapter 4); (2) ocean acidification, caused by increases in atmospheric $\mathrm{CO}_{2}$ being absorbed into the oceans, which lowers coral growth rates (Bongaerts et al., 2010); and (3) reduction in oxygen concentration in the ocean due to a combination of changes in temperature-driven gas solubility (Whitney et al., 2007), ocean ventilation due to circulation changes, and biological cycling of organic material (Keeling et al., 2010). Quantifying these changes and understanding their impact on coral reef health will be important to understanding the impact of anthropogenic climate change on atolls.

In summary, the small land area and often low elevation of small island states make them particularly vulnerable to rising sea levels and impacts such as inundation, shoreline change, and saltwater intrusion into underground aquifers. Short record lengths and the inadequate resolution of current climate models to represent small island states limit the assessment of changes in extremes. There is insufficient evidence to assess observed trends and future projections in rainfall across the small island regions considered here. The reported increases in warm days and nights and decreases in cold days and nights are of medium confidence over the Caribbean and of low confidence over the Pacific and Indian Oceans. There is medium confidence in the projected temperature increases across the Caribbean. The unique situation of small islands states and their maritime environments leads to an additional emphasis on oceanic information to understand the impacts of climate change. The very likely contribution of mean sea level rise to increased coastal high water levels, coupled with the likely increase in tropical cyclone maximum wind speed, is a specific issue for tropical small island states.

exacerbated by increasing human-induced pressures (Nicholls et al., 2007). However it was also noted that since coasts are dynamic systems, adaptation to climate change required understanding of processes operating on decadal to century time scales, yet this understanding was least developed.

Because of the diverse and complex nature of coastal impacts, assessments of the future impacts of climate change have focused on a wide range of questions and employed a diverse range of methods, making direct comparison of studies difficult (R.J. Nicholls, 2010). Two types of studies are examined here: the first are assessments, typically undertaken at the country or regional scale and which combine information on physical changes with the socioeconomic implications (e.g., Nicholls and de la Vega-Leinert, 2008); the second type are studies oriented around improved scientific understanding of the impacts of climate change. In terms of coastal assessments, Aunan and Romstad (2008) reported that Norway's generally steep and resistant coastlines contribute to a low physical susceptibility to accelerated sea level rise. Nicholls and de la Vega-Leinert (2008) reported that large parts of the coasts in Great Britain (including England, Wales, and Scotland) are already experiencing widespread sediment starvation and erosion, loss/degradation of coastal ecosystems, and significant exposure to coastal flooding. Lagoons, river deltas, and estuaries are assessed as being particularly vulnerable in Poland (Pruszak and Zawadzka, 2008). In Estonia, Kont et al. (2008) reported increased beach erosion, which is believed to be the result of increased storminess in the eastern Baltic Sea since 1954, combined with a decline in sea ice cover during the winter. Sterr (2008) reported that for Germany there is a high level of reliance on hard coastal protection against extreme sea level hazards, which will increase ecological vulnerability over time. In
France, the Atlantic coast Aquitaine region was considered more resilient to rising sea levels over the coming century because of the sediment storage in the extensive dune systems whereas the sandy coast regions of the Languedoc Roussillon region on the Mediterranean coast were considered more vulnerable because of narrow dune systems that are also highly urbanized (Vinchon et al., 2009). A coastal vulnerability assessment for Australia (Department of Climate Change, 2009) characterized future vulnerability in terms of coastal geomorphology, sediment type, and tide and wave characteristics, from which it concluded that the tropical northern coastline would be most sensitive to changes in tropical cyclone behavior while health of the coral reefs may also influence the tropical eastern coastline. The mid-latitude southern and eastern coastlines were expected to be most sensitive to changes in mean sea level, wave climate, and changes in storminess. A comparative study of the impact of sea level rise on coastal inundation across 84 developing countries showed that the greatest vulnerability to a $1 \mathrm{~m}$ sea level rise in terms of inundation of land area was located in East Asia and the Pacific, followed by South Asia, Latin America, and the Caribbean, the Middle East and North Africa, and finally sub-Saharan Africa (Dasgupta et al., 2009).

New models have been developed for the assessment of coastal vulnerability at the global to national level (Hinkel and Klein, 2009). At the local to regional scale, new techniques and approaches have also been developed to better quantify impacts from inundation due to future sea level rise. Bernier et al. (2007) evaluated spatial maps of extreme sea level for different return periods on a seasonal basis that were used to estimate seasonal risk of inundation under future sea level scenarios. Mclnnes et al. (2009a) developed spatial maps of storm tide 
and using a simple inundation model with high-resolution Light Detection and Ranging (LIDAR) data and a land subdivision database, identified the impact of inundation on several coastal towns along the southeastern Australian coastline under future sea level and wind speed scenarios. Probabilistic approaches have also been used to evaluate extreme sea level exceedance under uncertain future sea level rise scenarios. Purvis et al. (2008) constructed a probability distribution around the range of future sea level rise estimates and used Monte Carlo sampling to apply the sea level change to a two-dimensional coastal inundation model. They showed that by evaluating the possible flood-related losses in this framework they were able to represent spatially the higher losses associated with the low-frequency but high-impact inundation events instead of considering only a single midrange scenario. Hunter (2010) combined sea level extremes evaluated from observations with projections of sea level rise to 2100 and showed, for example, that planning levels in Sydney, Australia, would need to be increased substantially to cope with increased risk of flooding. Along the Portuguese coast, Andrade et al. (2007) found that projected future climate in the HadCM3 model would not affect wave height along this coastline but the projected rotation in wave direction would increase the net littoral drift and the erosional response. Along a section of the southeast coast of the United Kingdom, the effect of sea level rise, surge, and wave climate change on the inshore wave climate was evaluated and the frequency and height of extreme waves was projected to increase in the north of the domain (Chini et al., 2010). On the basis of modeling the 25 -year beach response along a stretch of the Portuguese coast to various climate change scenarios, Coelho et al. (2009) concluded that the projected stormier wave climate led to higher rates of beach erosion than mean sea level rise. Modeling of the evolution of soft rock shores with rising sea levels has revealed a relatively simple relationship between sea level rise and the equilibrium cliff profile (Walkden and Dickson, 2008).

To summarize, recent observational studies that identify trends and impacts at the coast are limited in regional coverage, which means there is low confidence, due to insufficient evidence, that anthropogenic climate change has been a major cause of any observed changes. However, recent coastal assessments at the national and regional scale and process-based studies have provided further evidence of the vulnerability of low-lying coastlines to rising sea levels and erosion, so that in the absence of adaptation there is high confidence that locations currently experiencing adverse impacts such as coastal erosion and inundation will continue to do so in the future due to increasing sea levels in the absence of changes in other contributing factors.

\subsubsection{Glacier, Geomorphological, and Geological Impacts}

Mountains are prone to mass movements including landslides, avalanches, debris flows, and flooding that can lead to disasters. Changes in the cryosphere affect such extremes, but also water supply and hydropower generation. Many of the world's high mountain ranges are situated at the margins of tectonic plates, increasing the possibility of potentially hazardous interactions between climatic and geological processes. The principal drivers are glacier ice mass loss, mountain permafrost degradation, and possible increases in the intensity of precipitation (Liggins et al., 2010; McGuire, 2010). The possible consequences are changes in mass movement on short contemporary time scales, and modulations of seismicity and volcanic activity on longer, century to millennium time scales.

The AR4 assessed that "the late 20th century glacier wastage likely has been a response to post-1970 warming" (Lemke et al., 2007). However, the impacts of glacier retreat on the natural physical system in the context of changes in extreme events were not assessed in detail. Additionally, the AR4 did not assess geomorphological and geological impacts that might result from anthropogenic climate change. The most studied change in the high-mountain environment has been the retreat of glaciers (Paul et al., 2004; Kaser et al., 2006; Larsen et al., 2007; Rosenzweig et al., 2007). Alpine glaciers around the world were at maximum extent by the end of the Little Ice Age ( 1850), and have retreated since then (Leclercq et al., 2011), with an accelerated decay during the past several decades (Zemp et al., 2007). Most glaciers have retreated since the mid-19th century (Francou et al., 2000; Cullen et al., 2006; Thompson et al., 2006; Larsen et al., 2007; Schiefer et al., 2007; Paul and Haeberli, 2008). Rates of retreat that exceed historical experience and internal (natural) variability have become apparent since the beginning of the 21st century (Reichert et al., 2002; Haeberli and Hohmann, 2008).

Outburst floods from lakes dammed by glaciers or unstable moraines [or 'glacial lake outburst floods' (GLOFs)] are commonly a result of glacier retreat and formation of lakes behind unstable natural dams (Clarke, 1982; Clague and Evans, 2000; Huggel et al., 2004; Dussaillant et al., 2010). In the past century, GLOFs have caused disasters in many highmountain regions of the world (Rosenzweig et al., 2007), including the Andes (Reynolds et al., 1998; Carey, 2005; Hegglin and Huggel, 2008), the Caucasus and Central Asia (Narama et al., 2006; Aizen et al., 2007), the Himalayas (Vuichard and Zimmermann, 1987; Richardson and Reynolds, 2000; Xin et al., 2008; Bajracharya and Mool, 2009; Osti and Egashira, 2009), North America (Clague and Evans, 2000; Kershaw et al., 2005), and the European Alps (Haeberli, 1983; Haeberli et al., 2001; Vincent et al., 2010). However, because GLOFs are relatively rare, it is unclear whether their frequency of occurrence is changing at either the regional or global scale. Clague and Evans (2000) argue that outburst floods from moraine-dammed lakes in North America may have peaked due to a reduction in the number of the lakes since the end of the Little Ice Age. In contrast, a small but not statistically significant increase of GLOF events was observed in the Himalayas over the period 1940 to 2000 (Richardson and Reynolds, 2000), but the event documentation may not be complete. Over the past several decades, human mitigation measures at unstable glacier lakes in the Himalaya and European Alps may have prevented some potential GLOF events (Reynolds, 1998; Haeberli et al., 2001).

Evidence of degradation of mountain permafrost and attendant slope instability has emerged from recent studies in the European Alps 
(Gruber and Haeberli, 2007; Huggel, 2009) and other mountain regions (Niu et al., 2005; Geertsema et al., 2006; Allen et al., 2011). This evidence includes several recent rock falls, rock slides, and rock avalanches in areas where permafrost thaw in steep bedrock is occurring. Landslides with volumes ranging up to a few million cubic meters have occurred in the Mont Blanc region (Barla et al., 2000), in Italy (Sosio et al., 2008; Huggel, 2009; Fischer et al., 2011), in Switzerland, and in British Columbia (Evans and Clague, 1998; Geertsema et al., 2006). Very large rock and ice avalanches with volumes of 30 to over 100 million $\mathrm{m}^{3}$ include the 2002 Kolka avalanche in the Caucasus (Haeberli et al., 2004; Kotlyakov et al., 2004; Huggel et al., 2005), the 2005 Mt. Steller rock avalanche in the Alaska Range (Huggel et al., 2008), the 2007 Mt. Steele ice and rock avalanche in the St. Elias Mountains, Yukon (Lipovsky et al., 2008), and the $2010 \mathrm{Mt}$. Meager rock avalanche and debris flow in the Coast Mountains of British Columbia.

Quantification of possible trends in the frequency of landslides and ice avalanches in mountains is difficult due to incomplete documentation of past events, especially those that happened before regular satellite observations became available. Nevertheless, there has been an apparent increase in large rock slides during the past two decades, and especially during the first years of the 21st century in the European Alps (Ravanel and Deline, 2011), in the Southern Alps of New Zealand (Allen et al., 2011), and in northern British Columbia (Geertsema et al., 2006) in combination with temperature increases, glacier shrinkage, and permafrost degradation.

Research, however, has not yet provided any clear indication of a change in the frequency of debris flows due to recent deglaciation. Debris flow activity at a local site in the Swiss Alps was higher during the 19th century than today (Stoffel et al., 2005). In the French Alps no significant change in debris flow frequency has been observed since the 1950s in terrain above elevations of 2,200 m (Jomelli et al., 2004). Processes not, or not directly, driven by climate, such as sediment yield, can also be important for changes in the magnitude or frequency of alpine debris flows (Lugon and Stoffel, 2010).

Debris flows from both glaciated and unglaciated volcanoes, termed lahars, can be particularly large and hazardous. Lahars produced by volcanic eruptions on the glacier-clad Nevado del Huila volcano in Colombia in 2007 and 2008 were the largest rapid mass flows on Earth in recent years. Similarly, large mass flows occur on ice-covered active volcanoes in Iceland (Björnsson, 2003), including Eyjafjallajökull in 2010. Large rock and ice avalanches, with volumes up to 30 million $\mathrm{m}^{3}$, have happened frequently (on average about one every four years) on the glaciated Alaskan volcano, lliamna, and are thought to be related to elevated volcanic heat flow and possibly meteorological conditions (Huggel et al., 2007). Glacier decay on active volcanoes can lead to a reduction of lahar hazards due to less potential meltwater available for lahar generation, but it is difficult to make a general conclusion as local conditions also play important roles. In 1998, intense rainfall mobilized pyroclastic material on the flanks of Vesuvius and Campi Flegrei volcanoes, feeding approximately 150 debris flows that damaged nearby communities and resulted in 160 fatalities (Bondi and Salvatori, 2003).
In the same year, intense precipitation associated with Hurricane Mitch triggered a small flank collapse at Casita volcano in Nicaragua. This slope failure transformed into debris flows that destroyed two towns and claimed 2,500 lives (Scott et al., 2005). Following the 1991 Pinatubo eruption in the Philippines, heavy rains associated with tropical storms moved large volumes of volcanic sediment. The sediment dammed rivers, causing massive flooding across the region that continued for several years after the eruption ended (Newhall and Punongbayan, 1996).

A variety of climate and weather events can have geomorphological and geological impacts. Warming and degradation of mountain permafrost affect slope stability through a reduction in the shear strength of ice-filled rock discontinuities. For example, the 2003 European summer heat wave (Section 3.3.1) caused rapid thaw and thickening of the active layer, triggering a large number of mainly small rock falls (Gruber et al., 2004; Gruber and Haeberli, 2007). Permafrost thaw in sediment such as in talus slopes may increase both the frequency and magnitude of debris flows (Zimmermann et al., 1997; Rist and Phillips, 2005). The frost table at the base of the active layer is a barrier to groundwater infiltration and can cause the overlying non-frozen sediment to become saturated. Snow cover can also affect debris flow activity by supplying additional water to the soil, increasing pore water pressure and initiating slope failure (Kim et al., 2004). Many of the largest debris flows in the Alps in the past 20 years were triggered by intense rainfall in summer or fall when the snowline was elevated (Rickenmann and Zimmermann, 1993; Chiarle et al., 2007). Warming may increase the flow speed of frozen bodies of sediment (Kääb et al., 2007; Delaloye et al., 2008; Roer et al., 2008). Rock slopes can fail after they have been steepened by glacial erosion or unloaded (debuttressed) following glacier retreat (Augustinus, 1995). Although it may take centuries or even longer for a slope to fail following glacier retreat, recent landslides demonstrate that some slopes can respond to glacier down-wasting within a few decades or less (Oppikofer et al., 2008). Twentieth-century warming may have penetrated some decameters into thawing steep rock slopes in high mountains (Haeberli et al., 1997). Case studies indicate that both small and large slope failures can be triggered by exceptionally warm periods of weeks to months prior to the events (Gruber et al., 2004; Huggel, 2009; Fischer et al., 2011).

The spatial and temporal patterns of precipitation, the intensity and duration of rainfall, and antecedent rainfall are important factors in triggering shallow landslides (Iverson, 2000; Wieczorek et al., 2005; Sidle and Ochiai, 2006). In some regions antecedent rainfall is probably a more important factor than rainfall intensity (Kim et al., 1991; Glade, 1998), whereas in other regions rainfall duration and intensity are the critical factors (Jakob and Weatherly, 2003). Landslides in temperate and tropical mountains that have no seasonal snow cover are not temperature-sensitive and may be more strongly influenced by human activities such as poor land use practices, deforestation, and overgrazing (Sidle and Ochiai, 2006).

Rock and ice avalanches on glaciated volcanoes can be triggered by heat generated by volcanic activity. Their incidence may increase with 
rising air and rock temperatures (Gruber and Haeberli, 2007) or during or following brief, anomalously warm events (Huggel et al., 2010) due to meltwater infiltration and shear strength reduction. Debuttressing effects due to glacier retreat can also destabilize or over-steepen slopes (Tuffen, 2010). Furthermore, on volcanoes, geothermal heat flow can enhance ice melting and thus create weak zones at the ice-bedrock interface; and hydrothermal alteration of rocks can decrease the slope stability (Huggel, 2009). On unglaciated high volcanoes in the Caribbean, Central America, Europe, Indonesia, the Philippines, and Japan, an increase in total rainfall or an increase in the frequency or magnitude of severe rainstorms (see Section 3.3.2) could cause more frequent debris flows by mobilizing unconsolidated, volcanic regolith and by raising porewater pressures, which could lead to deep-seated slope failure. Heavy rainfall events could also influence the behavior of active volcanoes. For example, Mastin (1994) attributes the violent venting of volcanic gases at Mount St. Helens between 1989 and 1991 to slope instability or accelerated growth of cooling fractures within the lava dome following rainstorms, and Matthews et al. (2002) link episodes of intense tropical rainfall with collapses of the Soufriere Hills lava dome on Montserrat in the Caribbean. It is well established that ice mass wastage following the end of the last glaciations led to increased levels of seismicity associated with post-glacial rebound of the lithosphere (e.g., Muir-Wood, 2000; Stewart et al., 2000). There has been a large reduction in glacier cover in southern Alaska. Sauber and Molnia (2004) reported several hundred meters vertical reduction. This ice reduction may be responsible for an increase in seismicity in the region where earthquake faults are at the threshold of failure (Sauber and Molnia, 2004; Doser et al., 2007). An increase in the frequency of small earthquakes in the Icy Bay area, also in southeast Alaska, is interpreted to be a crustal response to glacier wastage between 2002 and 2006 (Sauber and Ruppert, 2008). Largescale ice mass loss in glaciated volcanic terrain reduces the load on the crust and uppermost mantle, facilitating magma formation and its ascent into the crust (Jull and McKenzie, 1996) and allowing magma to reach the surface more easily (Sigmundsson et al., 2010). At the end of the last glaciation, this mechanism resulted in a more than 10-fold increase in the frequency of volcanic eruptions in Iceland (Sinton et al., 2005).

The AR4 projected that glaciers in mountains will lose additional mass over this century because more ice will be lost due to summer melting than is replenished by winter precipitation (Meehl et al., 2007b). The total area of glaciers in the European Alps may decrease by 20 to more than $50 \%$ by 2050 (Zemp et al., 2006; Huss et al., 2008). The projected glacier retreat in the 21st century may form new potentially unstable lakes. Probable sites of new lakes have been identified for some alpine glaciers (Frey et al., 2010). Rock slope and moraine failures may trigger damaging surge waves and outburst floods from these lakes. The temperature rise also will result in gradual degradation of mountain permafrost (Haeberli and Burn, 2002; Harris et al., 2009). The zone of warm permafrost (mean annual rock temperature approximately -2 to $0^{\circ} \mathrm{C}$ ), which is more susceptible to slope failures than cold permafrost, may rise in elevation a few hundred meters during the next 100 years (Noetzli and Gruber, 2009). This in turn may shift the zone of enhanced instability and landslide initiation toward higher-elevation slopes that in many regions are steeper, and therefore predisposed to failure. The response of bedrock temperatures to surface warming through thermal conduction will be slow, but warming will eventually penetrate to considerable depths in steep rock slopes (Noetzli et al., 2007). Other heat transport processes such as advection, however, may induce warming of bedrock at much faster rates (Gruber and Haeberli, 2007). The response of firn and ice temperatures to an increase in air temperature is faster and nonlinear (Haeberli and Funk, 1991; Suter et al., 2001; Vincent et al., 2007). Latent heat effects from refreezing meltwater can amplify the increase in air temperature in firn and ice (Huggel, 2009; Hoelzle et al., 2010). At higher temperatures, more ice melts and the strength of the remaining ice is lower; as a result, the frequency and perhaps size of ice avalanches may increase (Huggel et al., 2004; Caplan-Auerbach and Huggel, 2007). Warm extremes can trigger large rock and ice avalanches (Huggel et al., 2010).

Current low levels of seismicity in Antarctica and Greenland may be a consequence of ice-sheet loading, and isostatic rebound associated with accelerated deglaciation of these regions may result in an increase in earthquake activity, perhaps on time scales as short as 10 to 100 years (Turpeinen et al., 2008; Hampel et al., 2010). Future ice mass loss on glaciated volcanoes, notably in Iceland, Alaska, Kamchatka, the Cascade Range in the northwest United States, and the Andes, could lead to eruptions, either as a consequence of reduced load pressures on magma chambers or through increased magma-water interaction. Reduced ice load arising from future thinning of Iceland's Vatnajökull Ice Cap is projected to result in an additional $1.4 \mathrm{~km}^{3}$ of magma produced in the underlying mantle every century (Pagli and Sigmundsson, 2008). Ice unloading may also promote failure of shallow magma reservoirs with a potential consequence of a small perturbation of the natural eruptive cycle (Sigmundsson et al., 2010). Initially, ice thinning of $100 \mathrm{~m}$ or more on volcanoes with glaciers more than 150-m thick, such as Sollipulli in Chile, may cause more explosive eruptions, with increased tephra hazards (Tuffen, 2010). Additionally, the potential for edifice lateral collapse could be enhanced by loss of support previously provided by ice (Tuffen, 2010) or to elevated pore-water pressures arising from meltwater (Capra, 2006; Deeming et al., 2010). Ultimately the loss of ice cover on glaciated volcanoes may reduce opportunities for explosions arising from magma-ice interaction. The incidence of icesourced lahars may also eventually fall, although exposure of new surfaces of volcanic debris due to ice wastage may provide the raw material for precipitation-related lahars. The likelihood of both volcanic and non-volcanic landslides may also increase due to greater availability of water, which could destabilize slopes. Many volcanoes provide a ready source of unconsolidated debris that can be rapidly transformed into potentially hazardous lahars by extreme precipitation events. Volcanoes in coastal, near-coastal, or island locations in the tropics are particularly susceptible to torrential rainfall associated with tropical cyclones, and the rainfall rate associated with tropical cyclones is projected to increase though the number of tropical cyclones is projected to decrease or stay essentially unchanged (see Section 3.4.4). The impact of future large explosive volcanic eruptions may also be exacerbated by an 
increase in extreme precipitation events (see Section 3.3.2) that provide an effective means of transferring large volumes of unconsolidated ash and pyroclastic flow debris from the flanks of volcanoes into downstream areas.

Quantification of possible trends in the frequency of landslides and ice avalanches in mountains is difficult due to incomplete documentation of past events. There is high confidence that changes in heat waves, glacial retreat, and/or permafrost degradation will affect high mountain phenomena such as slope instabilities, mass movements, and glacial lake outburst floods, and medium confidence that temperature-related changes will influence bedrock stability. There is also high confidence that changes in heavy precipitation will affect landslides in some regions. There is medium confidence that high-mountain debris flows will begin earlier in the year because of earlier snowmelt, and that continued mountain permafrost degradation and glacier retreat will further decrease the stability of rock slopes. There is low confidence regarding future locations and timing of large rock avalanches, as these depend on local geological conditions and other non-climatic factors. There is low confidence in projections of an anthropogenic effect on phenomena such as shallow landslides in temperate and tropical regions, because these are strongly influenced by human activities such as poor land use practices, deforestation, and overgrazing. It is well established that ice mass wastage following the end of the last glaciations led to increased levels of seismicity, but there is low confidence in the nature of recent and projected future seismic responses to anthropogenic climate change.

\subsubsection{High-latitude Changes Including Permafrost}

Permafrost is widespread in Arctic, in subarctic, in ice-free areas of Antarctica, and in high-mountain regions, and permafrost regions occupy approximately 23 million $\mathrm{km}^{2}$ of land area in the Northern Hemisphere (Zhang et al., 1999). Melting of massive ground ice and thawing of ice-rich permafrost can lead to subsidence of the ground surface and to the formation of uneven topography known as thermokarst, having implications for ecosystems, landscape stability, and infrastructure performance (Walsh, 2005). See also Case Study 9.2.10 for discussion of the impacts of cold events in high latitudes. The active layer (nearsurface layer that thaws and freezes seasonally over permafrost) plays an important role in cold regions because most ecological, hydrological, biogeochemical, and pedogenic (soil-forming) activity takes place within it (Hinzman et al., 2005).

Observations show that permafrost temperatures have increased since the 1980s (IPCC, 2007b). Temperatures in the colder permafrost of northern Alaska, the Canadian Arctic, and Russia have increased up to $3^{\circ} \mathrm{C}$ near the permafrost table and up to 1 to $2^{\circ} \mathrm{C}$ at depths of 10 to $20 \mathrm{~m}$ (Osterkamp, 2007; Romanovsky et al., 2010; S.L. Smith et al., 2010) since the late 1970 s/early 1980s. Temperature increases have generally been less than
$1{ }^{\circ} \mathrm{C}$ in the warmer permafrost of the discontinuous permafrost zone of the polar regions (Osterkamp, 2007; Romanovsky et al., 2010; S.L. Smith et al., 2010), and also in the high-altitude permafrost of Mongolia and the Tibetan Plateau (Zhao et al., 2010). When the other conditions remain constant, active layer thickness is expected to increase in response to warming. Active layer thickness has increased by about $20 \mathrm{~cm}$ in the Russian Arctic between the early 1960s and 2000 (T. Zhang et al., 2005) and by up to $1.0 \mathrm{~m}$ over the Qinghai-Tibetan Plateau since the early 1980s (Wu and Zhang, 2010), with no significant trend in the North American Arctic since the early 1990s (Shiklomanov et al., 2010). However, over extreme warm summers, active layer thickness may increase substantially (Smith et al., 2009), potentially triggering activelayer detachment failures on slopes (Lewkowicz and Harris, 2005). Extensive thermokarst development has been found in Alaska (Jorgenson et al., 2006; Osterkamp et al., 2009), Canada (Vallée and Payette, 2007), and central Yakutia (Gavriliev and Efremov, 2003). Increased rates of retrogressive thaw slump activities have been reported on slopes over the Qinghai-Tibetan Plateau (Niu et al., 2005) and adjacent to tundra lakes over the Mackenzie Delta region of Canada (Lantz and Kokelj, 2008). Substantial expansion and deepening of thermokarst lakes was observed near Yakutsk with subsidence rates of 17 to $24 \mathrm{~cm} \mathrm{yr}^{-1}$ from 1992 to 2001 (Fedorov and Konstantinov, 2003). Satellite remote sensing data show that thaw lake surface area has increased in continuous permafrost regions and decreased in discontinuous permafrost regions (Smith et al., 2005). Coasts with ice-bearing permafrost that are exposed to the Arctic Ocean are very sensitive to permafrost degradation. Some Arctic coasts are retreating at a rapid rate of 2 to $3 \mathrm{~m} \mathrm{yr}^{-1}$ and the rate of erosion along Alaska's northeastern coastline has doubled over the past 50 years, related to declining sea ice extent, increasing sea surface temperature, rising sea level, thawing coastal permafrost, and possibly increases in storminess and waves (Jones et al., 2009; Karl et al., 2009)

Increases in air temperature are in part responsible for the observed increase in permafrost temperature over the Arctic and subarctic, but changes in snow cover also play a critical role (Osterkamp, 2005; Zhang, 2005; T. Zhang et al., 2005; S.L. Smith et al., 2010). Trends toward earlier snowfall in autumn and thicker snow cover during winter have resulted in a stronger snow insulation effect, and as a result a much warmer permafrost temperature than air temperature in the Arctic. On the other hand, permafrost temperature may decrease even if air temperature increases, if there is also a decrease in the duration and thickness of snow cover (Taylor et al., 2006). The lengthening of the thaw season and increases in summer air temperature have resulted in changes in active layer thickness. Model simulations have projected thickening of the active layer, a northward shift of the permafrost boundary, reductions in permafrost area, and an increase in permafrost temperature in the 21st century and beyond (Saito et al., 2007; Schaefer et al., 2011). The projected permafrost degradation may result in ancient carbon currently frozen in permafrost being released into the atmosphere, providing a positive feedback to the climate system (Schaefer et al., 2011). Expansion of lakes in the continuous permafrost zone may be due to thawing of ice-rich permafrost and melting of massive ground ice, while decreases in lake area in the discontinuous permafrost zone may be due to lake 
bottom drainage (Smith et al., 2005). Overall, increased air temperature over high latitudes is primarily responsible for the development of thermokarst terrains and thaw lakes.

In summary, it is likely that there has been warming of permafrost in recent decades. There is high confidence that permafrost temperatures will continue to increase, and that there will be increases in active layer thickness and reductions in the area of permafrost in the Arctic and subarctic.

\subsubsection{Sand and Dust Storms}

Sand and dust storms are widespread natural phenomena in many parts of the world. Heavy dust storms disrupt human activities. Dust aerosols in the atmosphere can cause a suite of health impacts including respiratory problems (Small et al., 2001). The long-range transport of dust can affect conditions at long distances from the dust sources, linking the biogeochemical cycles of land, atmosphere, and ocean (Martin and Gordon, 1988; Bergametti and Dulac, 1998; Kellogg and Griffin, 2006). For example, dust from the Saharan region and from Asia may reach North America and South America (McKendry et al., 2007). Some climate models have representations of dust aerosols (Textor et al., 2006). Climate variables that are most important to dust emission and transport such as soil moisture (see also Section 3.5.1), precipitation, wind, and vegetation cover are still subject to large uncertainties in climate model simulations. As a result, the sand and dust storm simulations have large uncertainties as well.

The Sahara (especially the Bodélé Depression in Chad) and east Asia have been recognized as the largest dust sources globally (Goudie, 2009). Over the few decades before the 1990s, the frequency of dust events increased in some regions such as the Sahel zone of Africa (Goudie and Middleton, 1992), and decreased in some other regions such as China (Zhang et al., 2003). There seems to be an increase in more recent years in China (Shao and Dong, 2006). Despite the importance of African dust, studies on long-term change in Sahel dust are limited. However, dust transported far away from the source region may provide some evidence of long-term changes in the Sahel region. The African dust transported to Barbados began to increase in the late 1960s and through the 1970s; transported dust reached a peak in the early 1980s but remains high into the present (Prospero and Lamb, 2003; Prospero et al., 2009).

Surface soil dust concentration during a sand and dust storm is controlled by a number of factors. The driving force for the production of dust storms is the surface wind associated with cold frontal systems sweeping across arid and semi-arid regions and lifting soil particles in the atmosphere. Dust emissions are also controlled by the surface conditions in source regions such as the desert coverage distributions, snow cover, and soil moisture. For example, in the Sahel region, the elevated high level of dust emission prior to the 1990s was related to the persistent drought during that time, and to long-term changes in the NAO (Ginoux et al., 2004; Chiapello et al., 2005; Engelstaedter et al., 2006), and perhaps to North Atlantic SST as well (Wong et al., 2008). Further evidence of the importance of climate on dust emission is that despite an increase of approximately 2 to $7 \%$ in desert areas in China over the four decades since 1960, dust storm frequency decreased in that period (Zhong, 1999). Studies on Asian soil dust production from 1960 to 2003 suggest that climatic variations have played a major role in the declining trends in dust emission and storm frequencies in China (Zhang et al., 2003; Zhou and Zhang, 2003; Zhao et al., 2004; Gong et al., 2006). Overall, changes in dust activity are affected by changes in the climate, such as wind and moisture conditions in the dust source regions. Changes in large-scale circulation play an additional role in the long-distance transport of dust. However, understanding of the physical mechanisms of the long-term trends in dust activity is not complete; for example, the relative importance of the various factors affecting dust frequency as outlined above is uncertain.

Future dust activity depends on two main factors: land use in the dust source regions, and climate both in the dust source region and largescale circulation that affects long distance dust transport. Studies on projected future dust activity are very limited. It is difficult to project future land use. Precipitation, soil moisture, and runoff have been projected to decrease in major dust source regions (Figure 10.12 in Meehl et al., 2007b). Thomas et al. (2005) suggest that dune fields in southern Africa can become active again, and sand will become significantly exposed and move, as a consequence of 21 st-century warming. A study based on simulations from two climate models also suggests increased desertification in arid and semi-arid China, especially in the second half of the 21st century (X.M. Wang et al., 2009). However, confident projected changes in wind are lacking (see Section 3.3.3).

In summary, there is low confidence in projecting future dust storm changes, although an increase could be expected where aridity increases. There is a lack of data and studies on past changes. There is also a lack of understanding of processes such as the relative importance of different climate variables affecting dust storms, as well as a high uncertainty in simulating important climate variables such as soil moisture, precipitation, and wind that affect dust storms. 


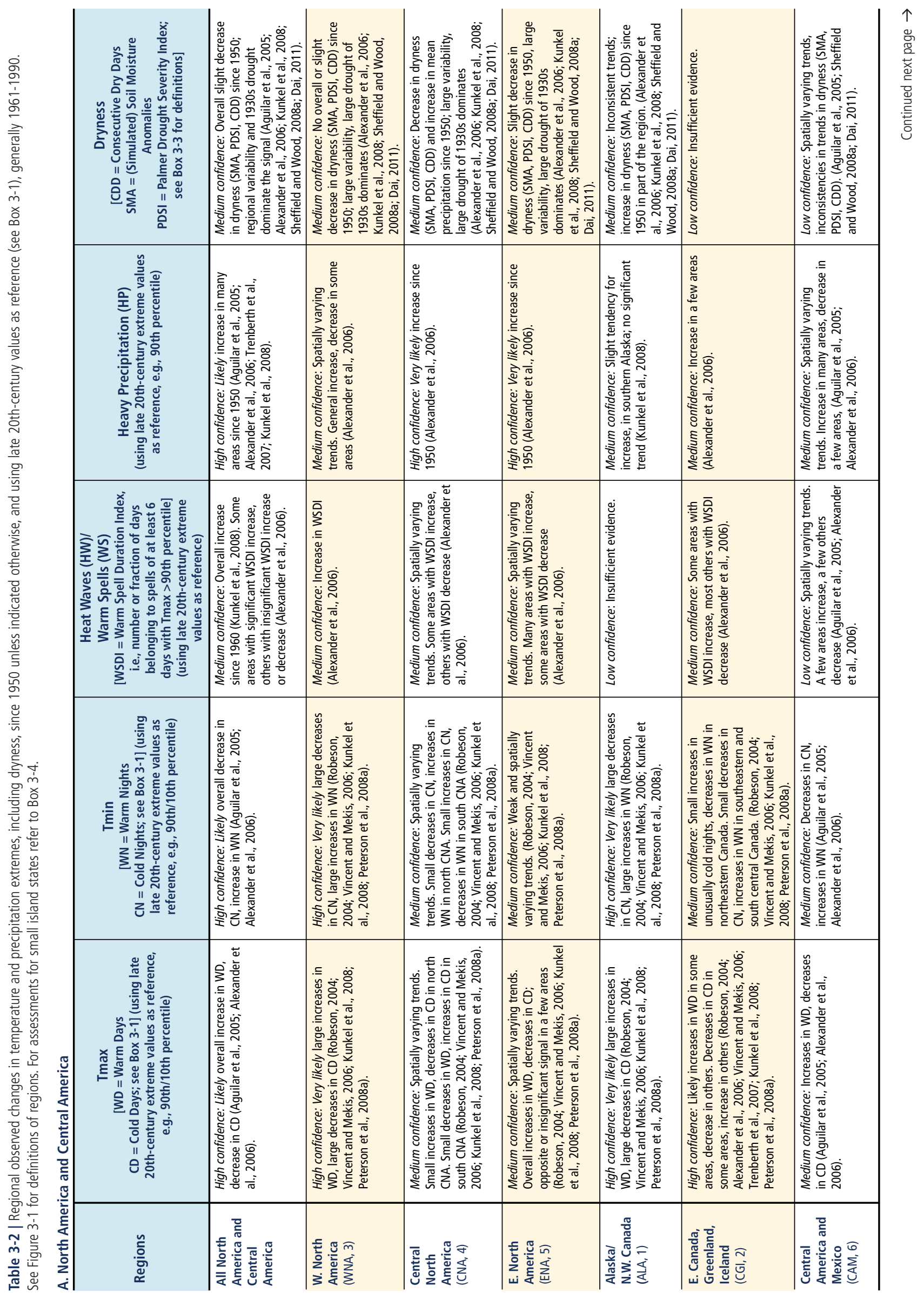




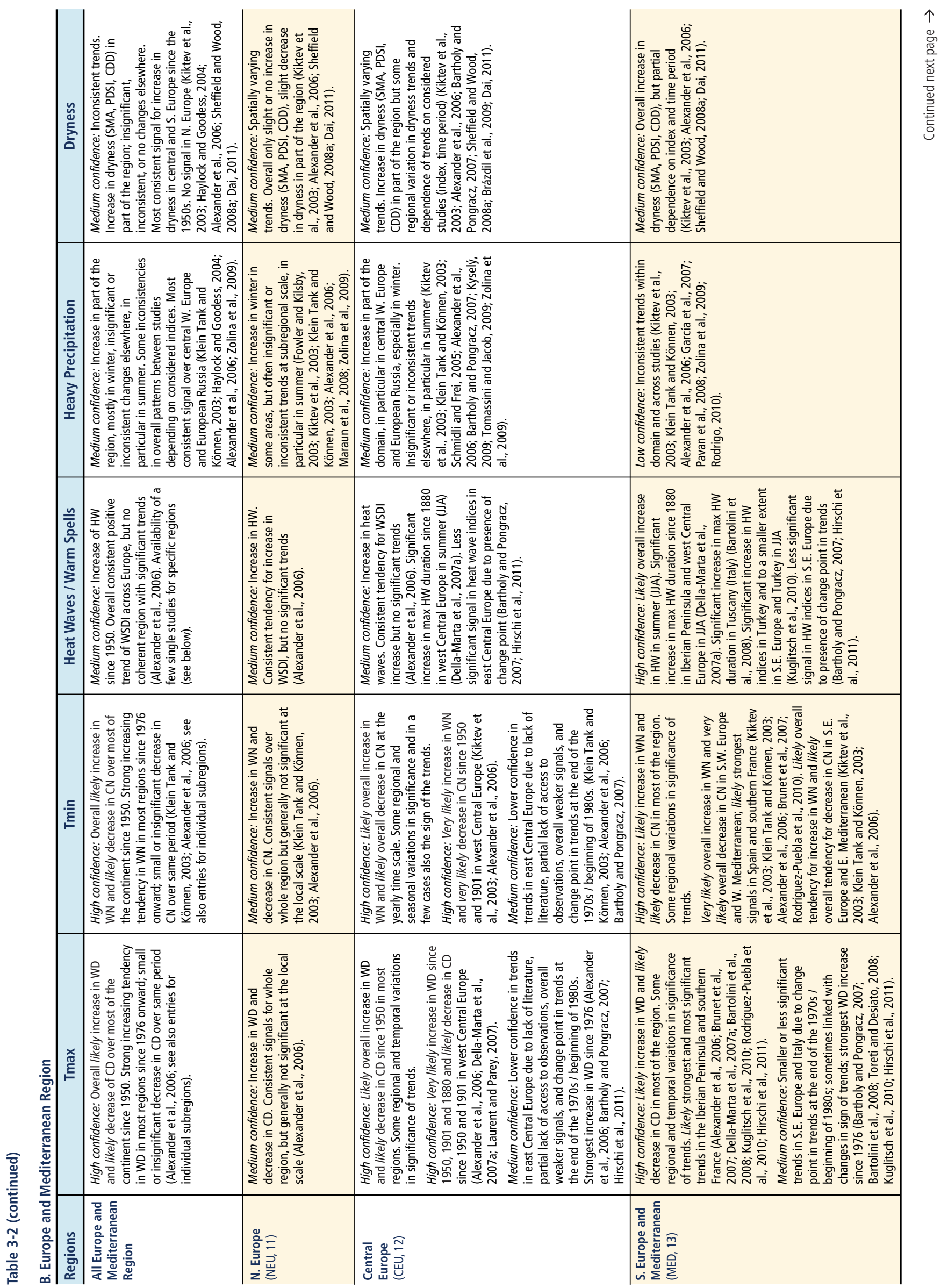




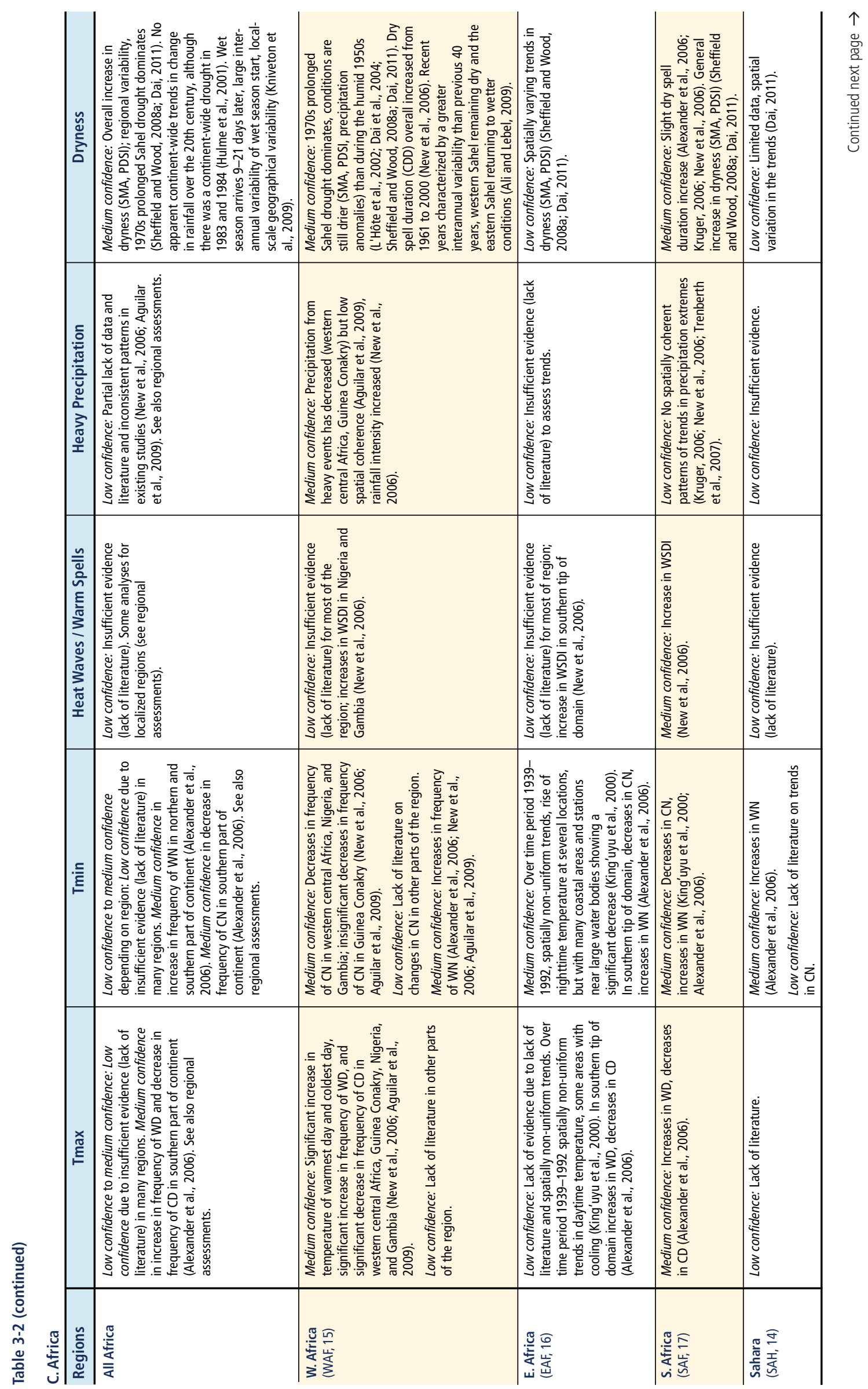




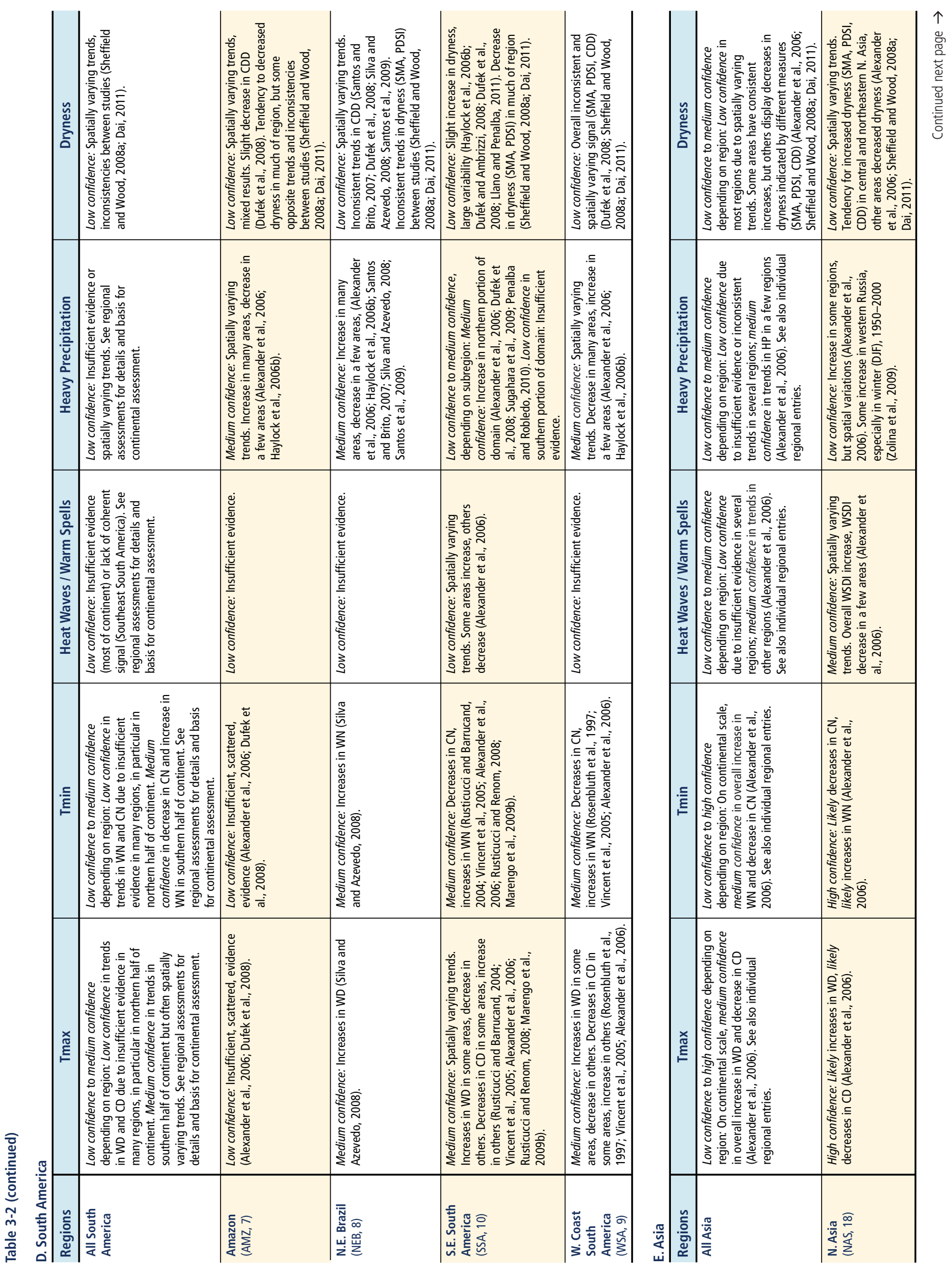




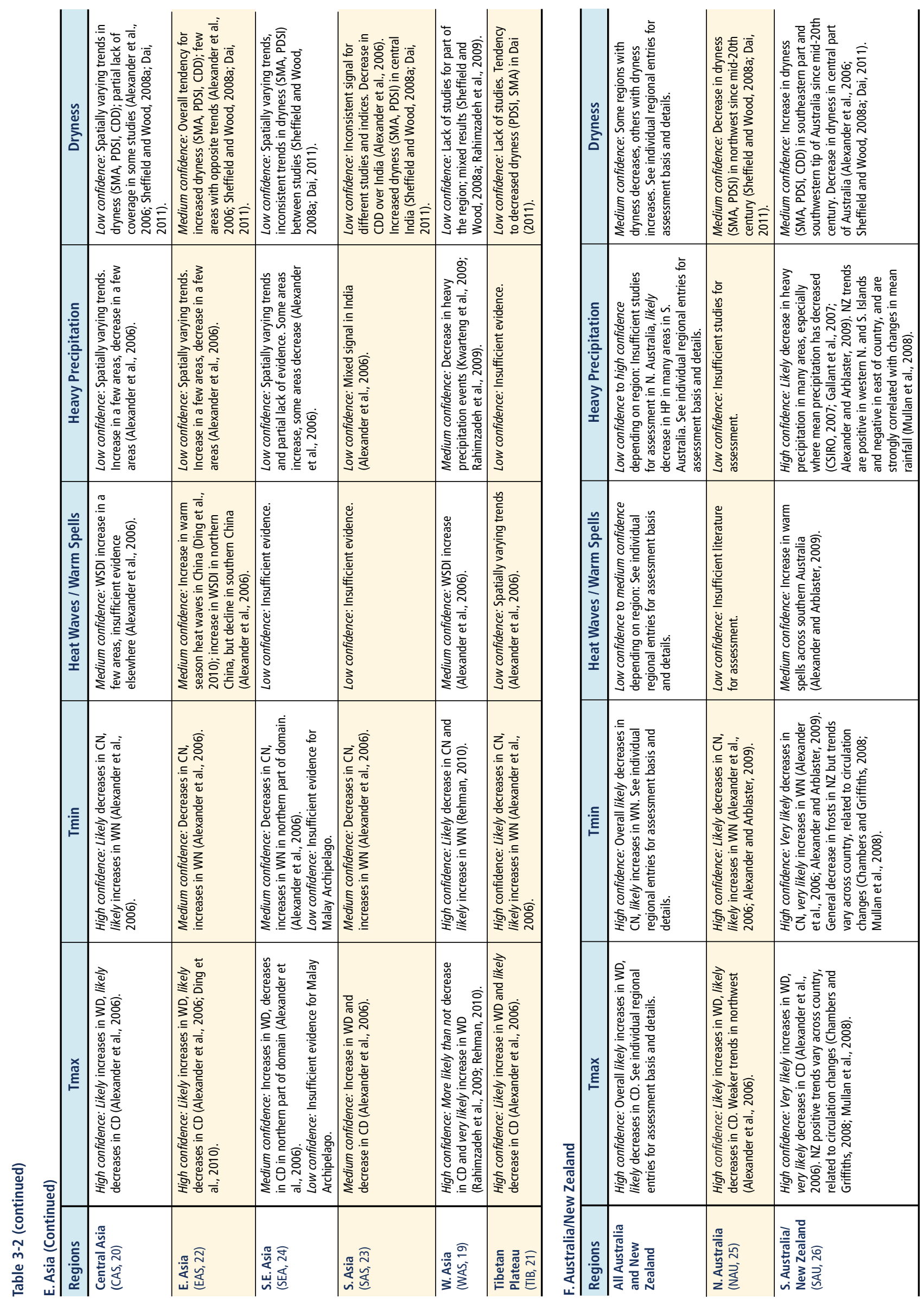




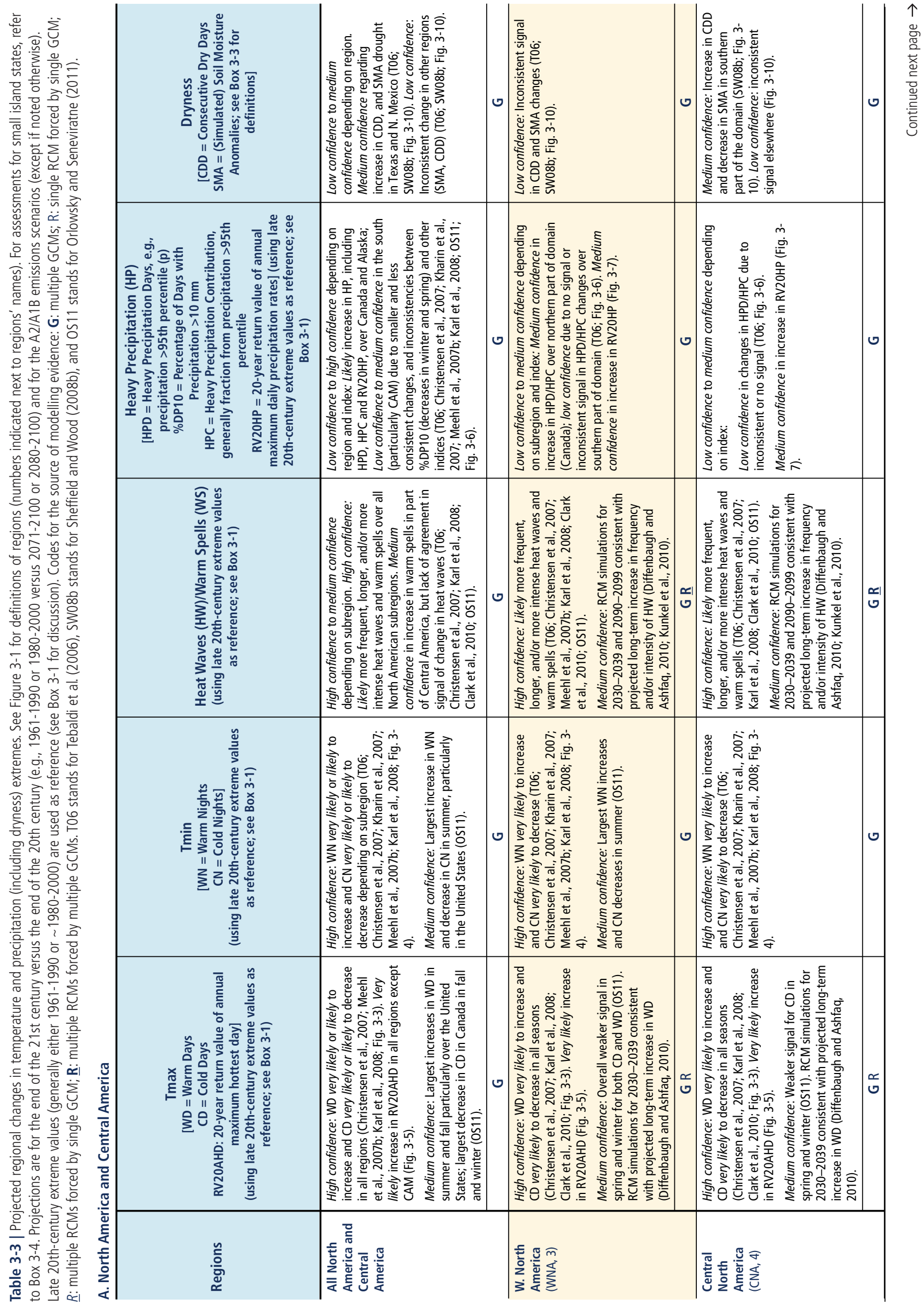




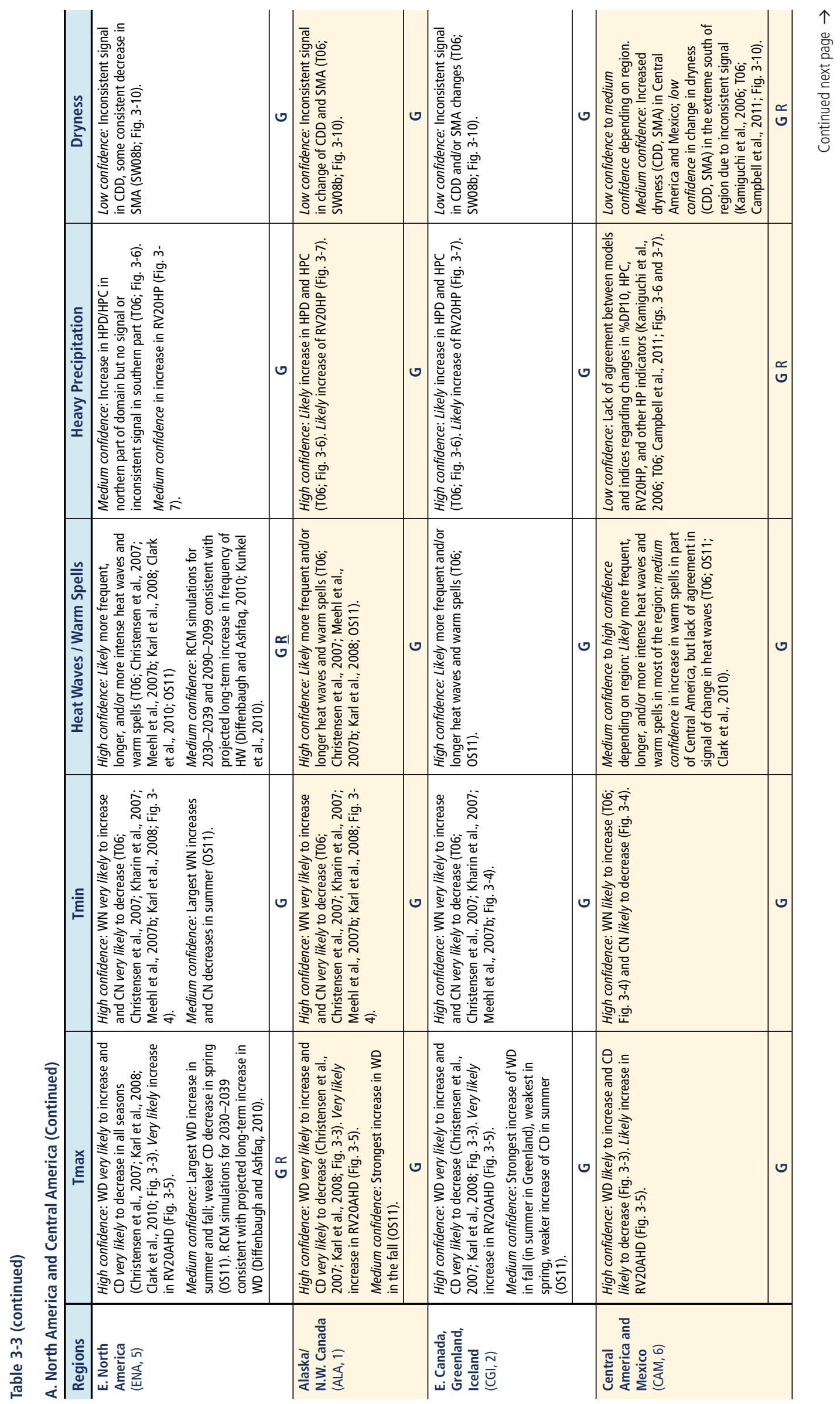




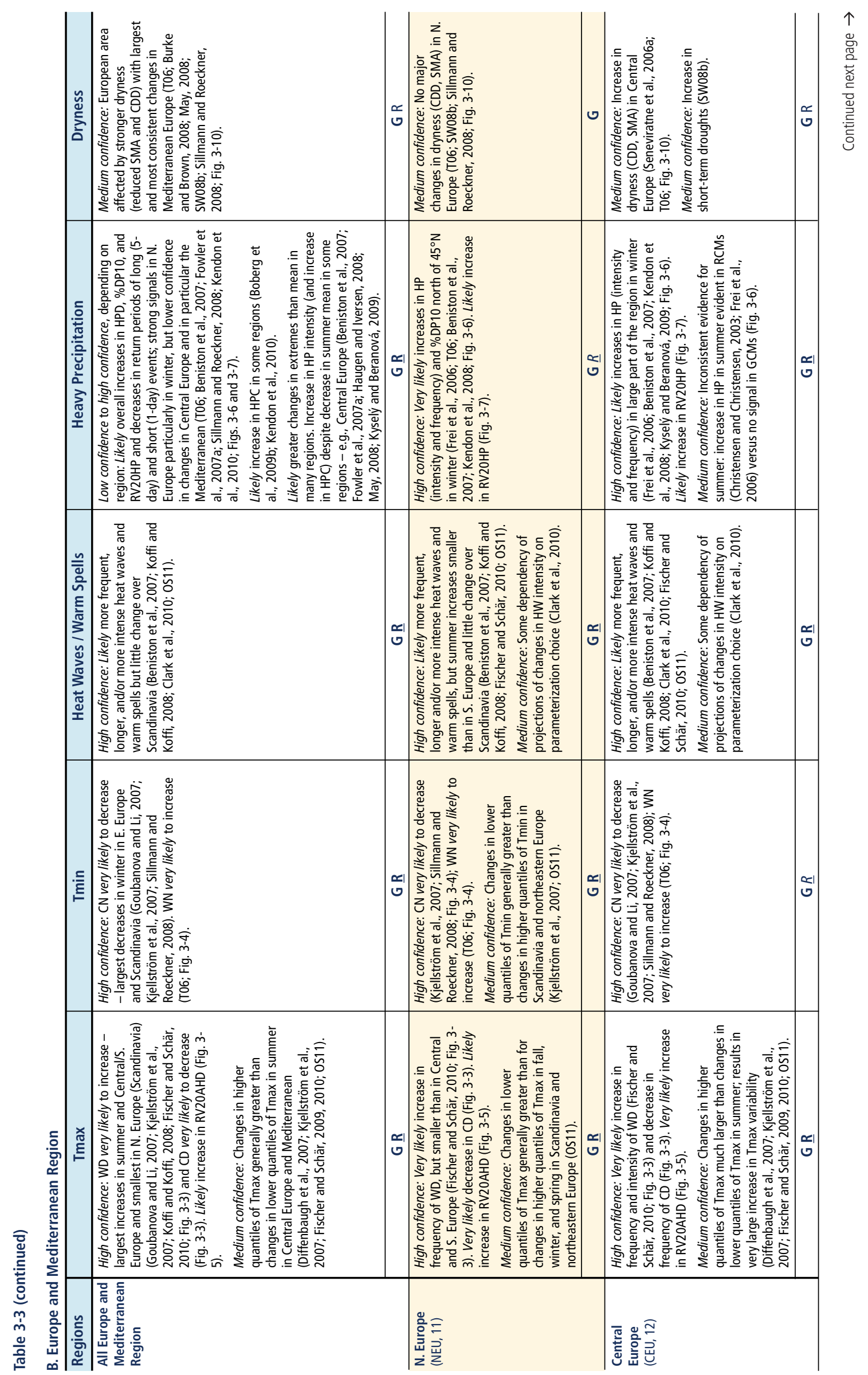




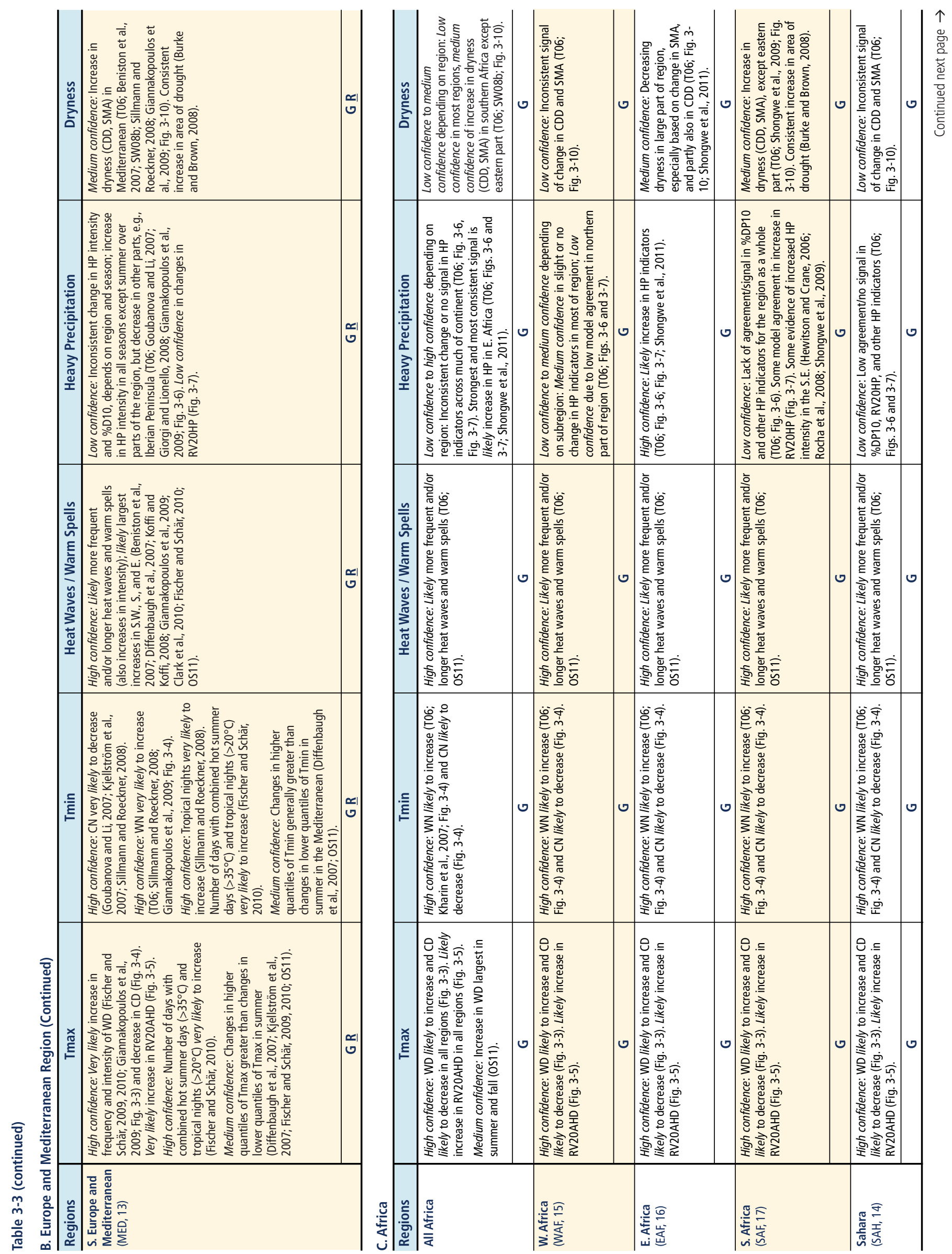




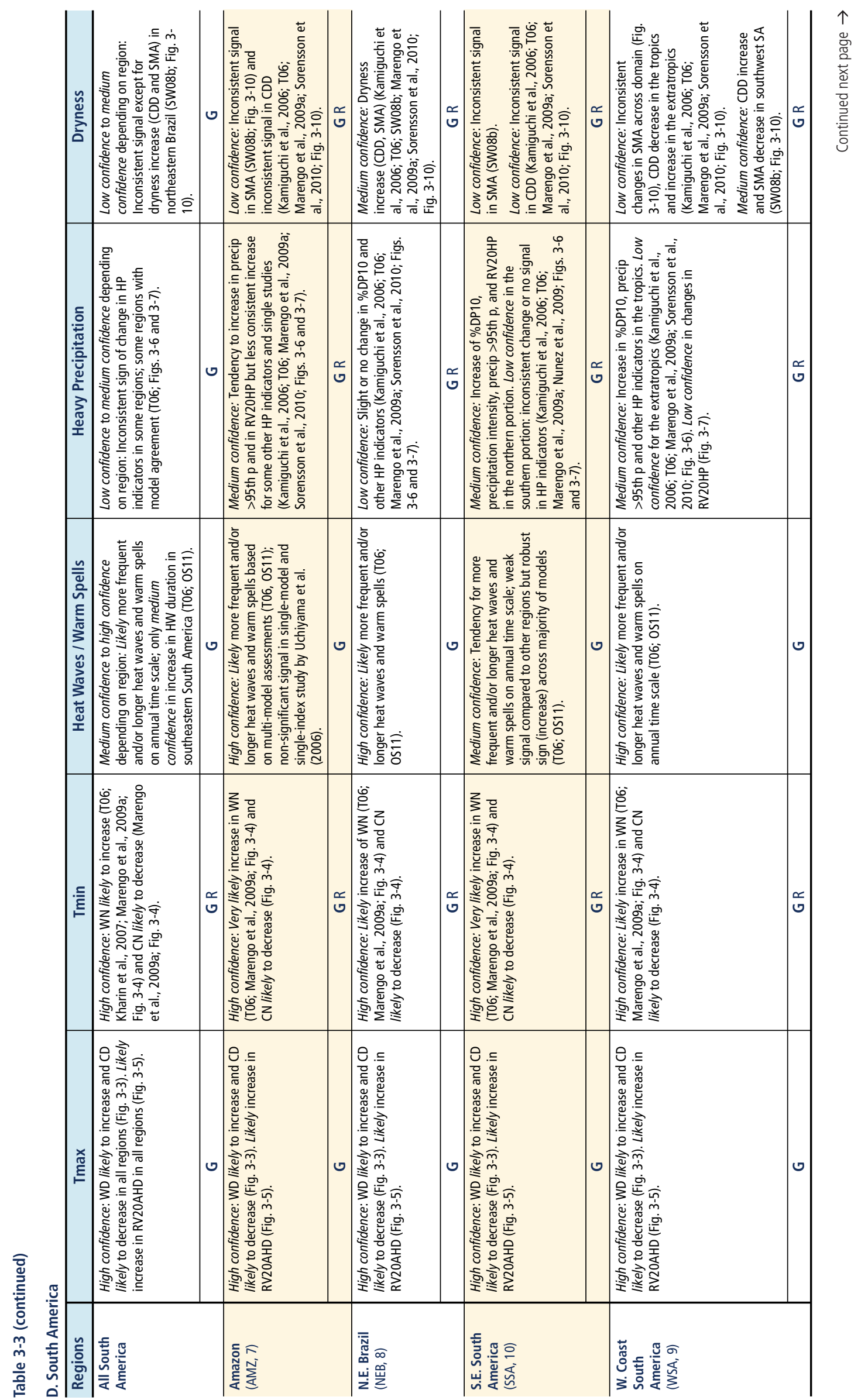




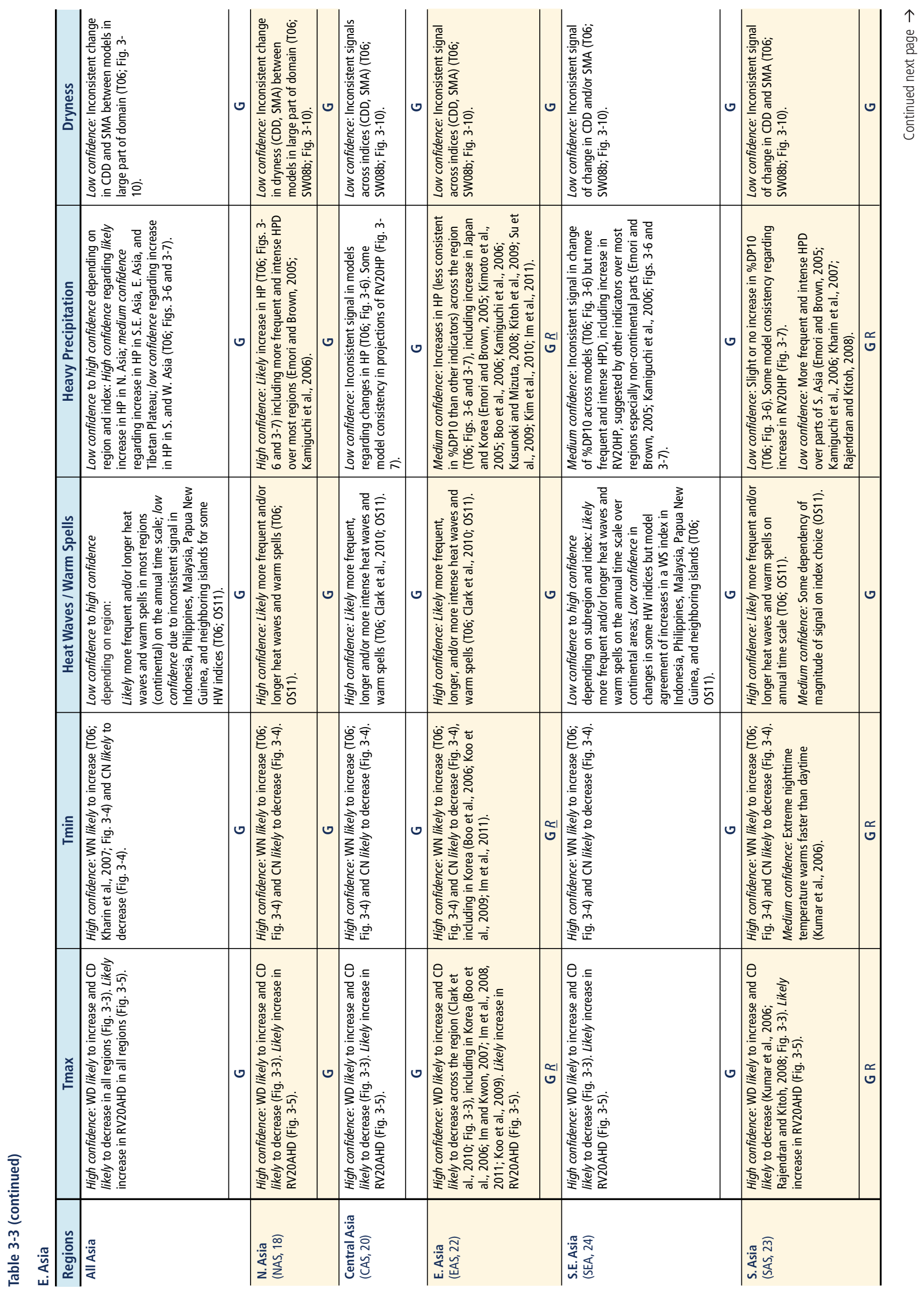




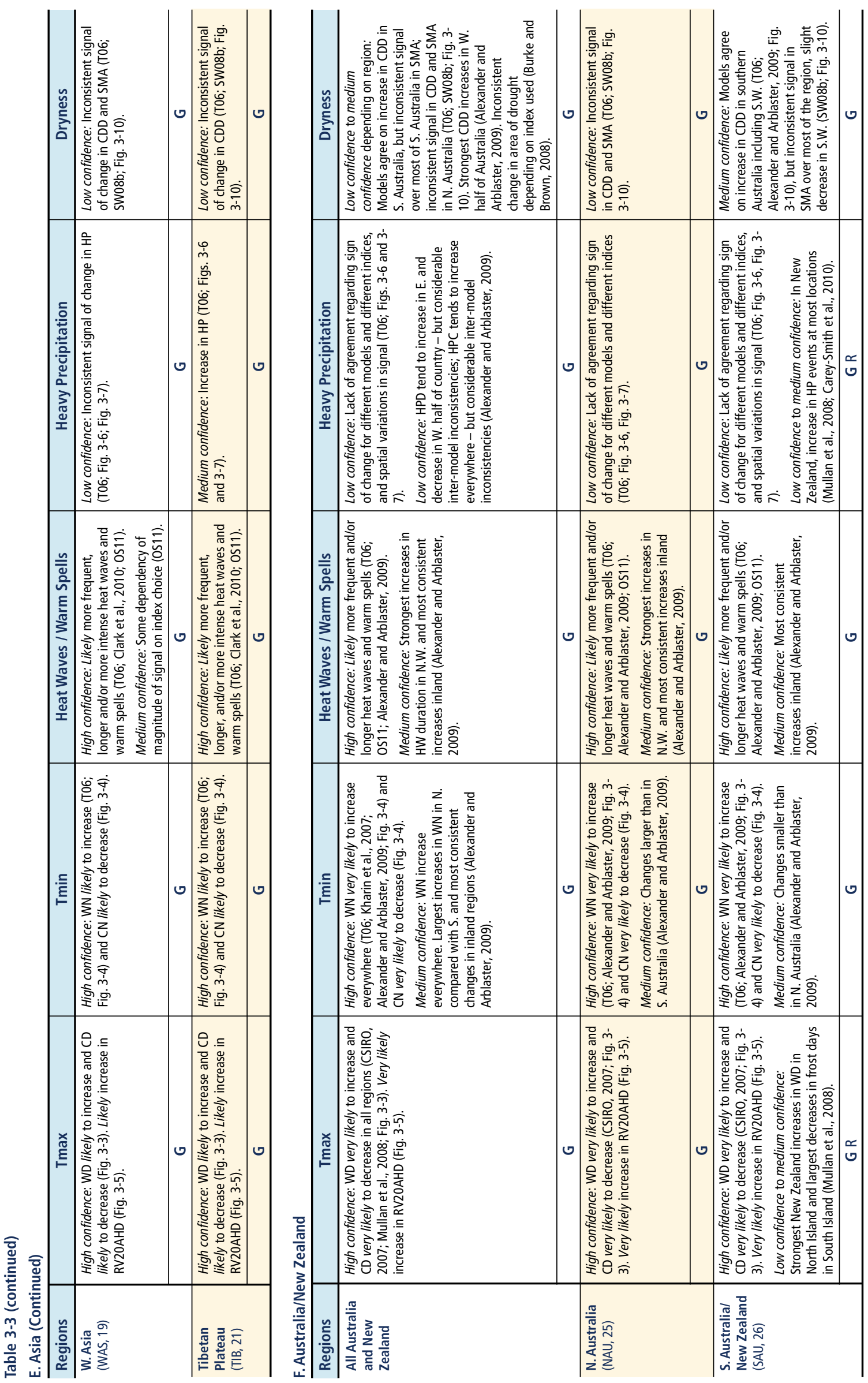




\section{References}

A digital library of non-journal-based literature cited in this chapter that may not be readily available to the public has been compiled as part of the IPCC review and drafting process, and can be accessed via either the IPCC Secretariat or IPCC Working Group II web sites.

Abbs, D., and T. Rafter, 2008: The Effect of Climate Change on Extreme Rainfall Events in the Western Port Region. CSIRO Marine and Atmospheric Research, Aspendale, Australia, $23 \mathrm{pp}$.

Abeysirigunawardena, D.S. and I.J. Walker, 2008: Sea level responses to climatic variability and change in northern British Columbia. Atmosphere-Ocean, 46(3), 277-296.

Abeysirigunawardena, D.S., E. Gilleland, D. Bronaugh, and W. Wong, 2009: Extreme wind regime responses to climate variability and change in the inner south coast of British Columbia, Canada. Atmosphere-Ocean, 47(1), 41-62.

Adams, P.N., D.L. Imnan, and N.E. Graham, 2008: Southern California deep-water wave climate: Characterization and application to coastal processes. Journal of Coastal Research, 24(4), 1022-1035.

Aguilar, E., T.C. Peterson, P. Ramírez Obando, R. Frutos, J.A. Retana, M. Solera, J. Soley, I. González García, R.M. Araujo, A. Rosa Santos, V.E. Valle, M. Brunet, L. Aguilar, L. Álvarez, M. Bautista, C. Castañón, L. Herrara, E. Ruano, J.J. Sinay, E. Sánchez, G.I. Hernández Oviedo, F. Obed, J.E. Salgado, J.L. Vázquez, M. Baca, M. Gutiérrez, C. Centella, J. Espinosa, D. Martinez, B. Olmedo, C.E. Ojeda Espinoza, R. Núñez, M. Haylock, H. Benavides, and R. Mayorga, 2005: Changes in precipitation and temperature extremes in Central America and northern South America, 19612003. Journal of Geophysical Research - Atmospheres, 110, D23107.

Aguilar, E., A. Aziz Barry, M. Brunet, L. Ekang, A. Fernandes, M. Massoukina, J. Mbah, A. Mhanda, D.J. do Nascimento, T.C. Peterson, M. Thamba Umba, M. Tomou, and $X$. Zhang, 2009: Changes in temperature and precipitation extremes in western central Africa, Guinea Conakry, and Zimbabwe, 1955-2006. Journal of Geophysical Research - Atmospheres, 114, D02115.

Aizen, V.B., V.A. Kuzmichenok, A.B. Surazakov, and E.M. Aizen, 2007: Glacier changes in the Tien Shan as determined from topographic and remotely sensed data. Global and Planetary Change, 56(3-4), 328-340.

Alexander, L.V. and J.M. Arblaster, 2009: Assessing trends in observed and modelled climate extremes over Australia in relation to future projections. International Journal of Climatology, 29(3), 417-435.

Alexander, L.V. and S. Power, 2009: Severe storms inferred from 150 years of subdaily pressure observations along Victoria's "Shipwreck Coast." Australian Meteorological and Oceanographic Journal, 58(2), 129-133.

Alexander, L.V., X. Zhang, T.C. Peterson, J. Caesar, B. Gleason, A.M.G. Klein Tank, M. Haylock, D. Collins, B. Trewin, F. Rahimzadeh, A. Tagipour, K.R. Kumar, J. Revadekar, G. Griffiths, L. Vincent, D.B. Stephenson, J. Burn, E. Aguilar, M. Brunet, M. Taylor, M. New, P. Zhai, M. Rusticucci, and J.L. Vazquez-Aguirre, 2006: Global observed changes in daily climate extremes of temperature and precipitation. Journal of Geophysical Research - Atmospheres, 111, D05109.

Alexander, L.V., P. Uotila, and N. Nicholls, 2009: Influence of sea surface temperature variability on global temperature and precipitation extremes. Journal of Geophysical Research - Atmospheres, 114, D18116.

Alexander, L.V., X.L. Wang, H. Wan, and B. Trewin, 2011: Significant decline in storminess over southeast Australia since the late 19th century. Australian Meteorological and Oceanographic Journal, 61(1), 23-30.

Ali, A. and T. Lebel, 2009: The Sahelian standardized rainfall index revisited. International Journal of Climatology, 29(12), 1705-1714.

Allamano, P., P. Claps, and F. Laio, 2009: Global warming increases flood risk in mountainous areas. Geophysical Research Letters, 36, L24404.

Allan, J.C. and P.D. Komar, 2006: Climate controls on US West Coast erosion processes. Journal of Coastal Research, 22(3), 511-529.
Allan, R., S. Tett, and L. Alexander, 2009: Fluctuations in autumn-winter severe storms over the British Isles: 1920 to present. International Journal of Climatology, 29(3), 357-371.

Allan, R.P. and B.J. Soden, 2008: Atmospheric warming and the amplification of precipitation extremes. Science, 321(5895), 1481-1484.

Allen, M., 2003: Liability for climate change: will it ever be possible to sue anyone for damaging the climate? Nature, 421(6926), 891-892.

Allen, M.R., D.J. Frame, C. Huntingford, C.D. Jones, J.A. Lowe, M. Meinshausen, and N. Meinshausen, 2009: Warming caused by cumulative carbon emissions towards the trillionth tonne. Nature, 458(7242), 1163-1166.

Allen, S.K., S.C. Cox, and I.F. Owens, 2011: Rock-avalanches and other landslides in the central Southern Alps of New Zealand: A regional assessment of possible climate change impacts. Landslides, 8(1), 33-48.

An, S.I., A. Timmermann, L. Bejarano, F.F. Jin, F. Justino, Z. Liu, and A.W. Tudhope, 2004: Modeling evidence for enhanced El Niño-Southern Oscillation amplitude during the Last Glacial Maximum. Paleoceanography, 19, PA4009.

Anders, I., and B. Rockel, 2009: The influence of prescribed soil type distribution on the representation of present climate in a regional climate model. Climate Dynamics, 33(2-3), 177-186.

Andersen, O.B., S.I. Seneviratne, J. Hinderer, and P. Viterbo, 2005: GRACE-derived terrestrial water storage depletion associated with the 2003 European heat wave. Geophysical Research Letters, 32, L18405.

Anderson, C.J., R.W. Arritt, E.S. Takle, Z.T. Pan, W.J. Gutowski, F.O. Otieno, R. da Silva, D. Caya, J.H. Christensen, D. Luthi, M.A. Gaertner, C. Gallardo, F. Giorgi, S.Y. Hong, C. Jones, H.M.H. Juang, J.J. Katzfey, W.M. Lapenta, R. Laprise, J.W. Larson, G.E. Liston, J.L. McGregor, R.A. Pielke, J.O. Roads, and J.A. Taylor, 2003: Hydrological processes in regional climate model simulations of the central United States flood of June-July 1993. Journal of Hydrometeorology, 4(3), 584-598.

Andrade, C., H.O. Pires, R. Taborda, and M.C. Freitas, 2007: Projecting future changes in wave climate and coastal response in Portugal by the end of the 21st century. Journal of Coastal Research, SI50, 253-257.

Andreadis, K.M. and D.P. Lettenmaier, 2006: Trends in 20th century drought over the continental United States. Geophysical Research Letters, 33, L10403.

Andreadis, K.M., E.A. Clark, A.W. Wood, A.F. Hamlet, and D.P. Lettenmaier, 2005: Twentieth-century drought in the conterminous United States. Journal of Hydrometeorology, 6(6), 985-1001.

Arblaster, J.M., G.A. Meehl, and D.J. Karoly, 2011: Future climate change in the Southern Hemisphere: Competing effects of ozone and greenhouse gases. Geophysical Research Letters, 38, L02701.

Arriaga-Ramírez, S. and T. Cavazos, 2010: Regional trends of daily precipitation indices in Northwest Mexico and the Southwest United States. Journal of Geophysical Research - Atmospheres, 115, D14111.

Aryal, S.K., B.C. Bates, E.D. Campbell, Y. Li, M.J. Palmer, and N.R. Viney, 2009: Characterizing and modeling temporal and spatial trends in rainfall extremes. Journal of Hydrometeorology, 10(1), 241-253.

Ashfaq, M., Y. Shi, W.W. Tung, R.J. Trapp, X. Gao, S.J. Pal, and N.S. Diffenbaugh, 2009: Suppression of south Asian summer monsoon precipitation in the 21st century. Geophysical Research Letters, 36, L01704.

Asokan, S.M., and D. Dutta, 2008: Analysis of water resources in the Mahanadi River Basin, India under projected climate conditions. Hydrological Processes, 22(18), 3589-3603.

Augustinus, P., 1995: Rock mass strength and the stability of some glacial valley slopes. Zeitschrift Für Geomorphologie, 39(1), 55-68.

Aunan, K., and B. Romstad, 2008: Strong coasts and vulnerable communities: Potential implications of accelerated sea-level rise for Norway. Journal of Coastal Research, 24(2), 403-409.

Bader, J., M.D.S. Mesquita, K.I. Hodges, N. Keenlyside, S. Østerhus, and M. Miles, 2011: A review on Northern Hemisphere sea-ice, storminess and the North Atlantic Oscillation: Observations and projected changes. Atmospheric Research, 101(4), 809-834, doi:10.1016/j.atmosres.2011.1004.1007.

Bajracharya, S.R. and P. Mool, 2009: Glaciers, glacial lakes and glacial lake outburst floods in the Mount Everest region, Nepal. Annals of Glaciology, 50(53), 81-86. 
Ballester, J., F. Giorgi, and X. Rodo, 2010: Changes in European temperature extremes can be predicted from changes in PDF central statistics. Climatic Change, 98(1-2), 277-284.

Barla, G., F. Dutto, and G. Mortara, 2000: Brenva glacier rock avalanche of 18 January 1997 on the Mount Blanc range, northwest Italy. Landslide News, 13, 2-5.

Barnett, D.N., S.J. Brown, J.M. Murphy, D.M.H. Sexton, and M.J. Webb, 2006: Quantifying uncertainty in changes in extreme event frequency in response to doubled $\mathrm{CO}_{2}$ using a large ensemble of GCM simulations. Climate Dynamics, 26(5), 489-511.

Barnett, T.P., D.W. Pierce, H.G. Hidalgo, C. Bonfils, B.D. Santer, T. Das, G. Bala, A.W. Wood, T. Nozawa, A.A. Mirin, D.R. Cayan, and M.D. Dettinger, 2008: Humaninduced changes in the hydrology of the western United States. Science, 319(5866), 1080-1083.

Barring, L. and K. Fortuniak, 2009: Multi-indices analysis of southern Scandinavian storminess 1780-2005 and links to interdecadal variations in the NW EuropeNorth Sea region. International Journal of Climatology, 29(3), 373-384.

Barring, L., and H. von Storch, 2004: Scandinavian storminess since about 1800. Geophysical Research Letters, 31, L20202.

Barring, L., T. Holt, M.L. Linderson, M. Radziejewski, M. Moriondo, and J.P. Palutikof, 2006: Defining dry/wet spells for point observations, observed area averages, and regional climate model gridboxes in Europe. Climate Research, 31(1), 35-49.

Barriopedro, D., E.M. Fischer, J. Luterbacher, R.M. Trigo, and R. García-Herrera, 2011: The hot summer of 2010: Redrawing the temperature record map of Europe. Science, 332(6026), 220-224.

Barros, V., L. Chamorro, G. Coronel, and J. Baez, 2004: The major discharge events in the Paraguay River: Magnitudes, source regions, and climate forcings. Journal of Hydrometeorology, 5(6), 1161-1170.

Bartholy, J., and R. Pongracz, 2007: Regional analysis of extreme temperature and precipitation indices for the Carpathian Basin from 1946 to 2001. Global and Planetary Change, 57, 83-95.

Bartolini, G., M. Morabito, A. Crisci, D. Grifoni, T. Torrigiani, M. Petralli, G. Maracchi, and S. Orlandini, 2008: Recent trends in Tuscany (Italy) summer temperature and indices of extremes. International Journal of Climatology, 28(13), 1751-1760.

Bates, B.C., Z.W. Kundzewics, S. Wu, and J.P. Palutikof, 2008: Climate Change and Water. Technical Paper of the Intergovernmental Panel on Climate Change. IPCC Secretariat, Geneva, Switzerland, $210 \mathrm{pp}$.

Beaulant, A.L., B. Joly, O. Nuissier, S. Somot, V. Ducrocq, A. Joly, F. Sevault, M. Deque, and D. Ricard, 2011: Statistico-dynamical downscaling for Mediterranean heavy precipitation. Quarterly Journal of the Royal Meteorological Society, 137(656), 736-748.

Begueria, S., S.M. Vicente-Serrano, and M. Angulo-Martinez, 2010: A multiscalar global drought dataset: The SPElbase: A new gridded product for the analysis of drought variability and impacts. Bulletin of the American Meteorological Society, 91(10), 1351-1354.

Beirlant, J., Y. Goegebeur, J. Teugels, and J. Segers, 2004: Statistics of Extremes: Theory and Applications. John Wiley \& Sons, Chichester, West Sussex, England, $498 \mathrm{pp}$.

Beltaos, S. and T. Prowse, 2009: River-ice hydrology in a shrinking cryosphere. Hydrological Processes, 23(1), 122-144.

Bender, M.A., T.R. Knutson, R.E. Tuleya, J.J. Sirutis, G.A. Vecchi, S.T. Garner, and I.M. Held, 2010: Modeled impact of anthropogenic warming on the frequency of intense Atlantic hurricanes. Science, 327(5964), 454-458.

Benestad, R.E., 2001: A comparison between two empirical downscaling strategies. International Journal of Climatology, 21(13), 1645-1668.

Benestad, R.E., 2003: How often can we expect a record event? Climate Research, 25(1), 3-13

Benestad, R.E., 2006: Can we expect more extreme precipitation on the monthly time scale? Journal of Climate, 19(4), 630-637.

Benestad, R.E., 2007: Novel methods for inferring future changes in extreme rainfall over Northern Europe. Climate Research, 34(3), 195-210.

Benestad, R.E., and J.E. Haugen, 2007: On complex extremes: flood hazards and combined high spring-time precipitation and temperature in Norway. Climatic Change, 85(3-4), 381-406
Benestad, R.E., I. Hanssen-Bauer, and E.J. Forland, 2007: An evaluation of statistical models for downscaling precipitation and their ability to capture long-term trends. International Journal of Climatology, 27(5), 649-665.

Bengtsson, L., S. Hagemann, and K.I. Hodges, 2004: Can climate trends be calculated from reanalysis data? Journal of Geophysical Research - Atmospheres, 109, D11111.

Bengtsson, L., K.I. Hodges, and E. Roeckner, 2006: Storm tracks and climate change. Journal of Climate, 19(15), 3518-3543.

Bengtsson, L., K.I. Hodges, M. Esch, N. Keenlyside, L. Kornblueh, J.J. Luo, and T. Yamagata, 2007: How may tropical cyclones change in a warmer climate? Tellus Series A - Dynamic Meteorology and Oceanography, 59(4), 539-561.

Bengtsson, L., K.I. Hodges, and N. Keenlyside, 2009: Will extratropical storms intensify in a warmer climate? Journal of Climate, 22(9), 2276-2301.

Beniston, M., 2004: The 2003 heat wave in Europe: A shape of things to come? An analysis based on Swiss climatological data and model simulations. Geophysical Research Letters, 31, L02202.

Beniston, M., 2009a: Decadal-scale changes in the tails of probability distribution functions of climate variables in Switzerland. International Journal of Climatology, 29(10), 1362-1368.

Beniston, M., 2009b: Trends in joint quantiles of temperature and precipitation in Europe since 1901 and projected for 2100. Geophysical Research Letters, 36, L07707.

Beniston, M., D.B. Stephenson, O.B. Christensen, C.A.T. Ferro, C. Frei, S. Goyette, K. Halsnaes, T. Holt, K. Jylha, B. Koffi, J. Palutikof, R. Schoell, T. Semmler, and K. Woth, 2007: Future extreme events in European climate: an exploration of regional climate model projections. Climatic Change, 81, 71-95.

Benito, G., A. Diez-Herrero, and M.F. de Villalta, 2003: Magnitude and frequency of flooding in the Tagus basin (Central Spain) over the last millennium. Climatic Change, 58(1-2), 171-192.

Benito, G., M. Barriendos, C. Llasat, M. Machado, and V. Thorndycraft, 2005: Impacts on natural hazards of climatic origin. Flood risk. In: A Preliminary General Assessment of the Impacts in Spain Due to the Effects of Climate Change [Moreno, J.M. (ed.)]. Ministry of Environment, Spain, pp. 507-527.

Benito, G., V.R. Thorndycraft, M. Rico, Y. Sanchez-Moya, and A. Sopena, 2008: Palaeoflood and floodplain records from Spain: Evidence for long-term climate variability and environmental changes. Geomorphology, 101(1-2), 68-77.

Bergametti, G. and F. Dulac, 1998: Mineral aerosols: Renewed interest for climate forcing and tropospheric chemistry studies. IGACtivities Newsletter, 11, 13-17.

Bernier, N.B., K.R. Thompson, J. Ou, and H. Ritchie, 2007: Mapping the return periods of extreme sea levels: Allowing for short sea level records, seasonality, and climate change. Global and Planetary Change, 57(1-2), 139-150.

Betts, R.A., O. Boucher, M. Collins, P.M. Cox, P.D. Falloon, N. Gedney, D.L. Hemming, C. Huntingford, C.D. Jones, D.M.W. Sexton, and M.J. Web, 2007: Projected increase in continental runoff due to plant responses to increasing carbon dioxide. Nature, 448(7157), 1037-1041.

Bevan, S.L.N., P. R. J. North, W.M.F. Grey, S.O. Los, and S.E. Plummer, 2009: Impact of atmospheric aerosol from biomass burning on Amazon dry-season drought Journal of Geophysical Research - Atmospheres, 114, D09204.

Bhutiyani, M.R., V.S. Kale, and N.J. Pawar, 2008: Changing streamflow patterns in the rivers of northwestern Himalaya: Implications of global warming in the 20th century. Current Science, 95(5), 618-626.

Biasutti, M., A.H. Sobel, and S.J. Camargo, 2009: The role of the Sahara Low in summertime Sahel rainfall variability and change in the CMIP3 models. Journal of Climate, 22(21), 5755-5771.

Bindoff, N.L., J. Willebrand, V. Artale, A. Cazenave, J.M. Gregory, S. Gulev, K. Hanawa, C. Le Quéré, S. Levitus, Y. Nojiri, C.K. Shum, L.D. Talley, and A.S. Unnikrishnan, 2007: Observations: Oceanic climate change and sea level. In: Climate Change 2007: The Physical Science Basis. Contribution of Working Group I to the Fourth Assessment Report of the Intergovernmental Panel on Climate Change [Solomon, S., D. Qin, M. Manning, Z. Chen, M. Marquis, K.B. Averyt, M. Tignor and H.L. Miller (eds.)]. Cambridge University Press, Cambridge, UK, pp. 385-432.

Bister, M. and K.A. Emanuel, 1998: Dissipative heating and hurricane intensity. Meteorology and Atmospheric Physics, Vienna, Austria, 65(3-4), 233-240. 
Bister, M. and K.A. Emanuel, 2002: Low frequency variability of tropical cyclone potential intensity 2. Climatology for 1982-1995. Journal of Geophysical Research - Atmospheres, 107(D22), 4621-4624.

Björnsson, H., 2003: Subglacial lakes and jökulhlaups in Iceland. Global and Planetary Change, 35(3-4), 255-271.

Blenkinsop, S. and H.J. Fowler, 2007a: Changes in drought frequency, severity and duration for the British Isles projected by the PRUDENCE regional climate models. Journal of Hydrology, 342, 50-71.

Blenkinsop, S. and H.J. Fowler, 2007b: Changes in European drought characteristics projected by the PRUDENCE regional climate models. International Journal of Climatology, 27(12), 1595-1610.

Blewitt, G., Z. Altamimi, J. Davis, R. Gross, C.-Y. Kuo, F.G. Lemoine, A.W. Moore, R.E. Neilan, H.-P. Plag, M. Rothacher, C.K. Shum, M.G. Sideris, T. Schöne, P. Tregoning, and S. Zerbini, 2010: Geodetic observations and global reference frame contributions to understanding sea-level rise and variability. In: Understanding Sea-Level Rise and Variability [Church, J.A., P.L. Woodworth, T. Aarup, and W.S. Wilson (eds.)]. Wiley-Blackwell, Chichester, UK, pp. 256-284.

Boberg, F., P. Berg, P. Thejll, W.J. Gutowski, and J.H. Christensen, 2009a: Improved confidence in climate change projections of precipitation evaluated using daily statistics from the PRUDENCE ensemble. Climate Dynamics, 32(7-8), 1097-1106.

Boberg, F., P. Berg, P. Thejll, W.J. Gutowski, and J.H. Christensen, 2009b: Improved confidence in climate change projections of precipitation further evaluated using daily statistics from the PRUDENCE ensemble. Climate Dynamics, 35(7-8), 1509-1520.

Boe, J., and L. Terray, 2008: Uncertainties in summer evapotranspiration changes over Europe and implications for regional climate change. Geophysical Research Letters, 35, L05702.

Boer, G.J., 2009: Changes in interannual variability and decadal potential predictability under global warming. Journal of Climate, 22, 3098-3109.

Bollasina, M., and S. Nigam, 2009: Indian Ocean SST, evaporation, and precipitation during the South Asian summer monsoon in IPCC-AR4 coupled simulations. Climate Dynamics, 33(7-8), 1017-1032.

Bonan, G.B., 2008: Forests and climate change: Forcing, feedbacks, and the climate benefit of forests. Science, 320, 1444-1449.

Bondi, F. and L. Salvatori, 2003: The 5-6 May 1998 mudflows in Campania, Italy. In: Lessons Learnt from Landslide Disasters in Europe [Hervas, J. (ed.)]. European Commission, Brussels, Belgium, pp. 102.

Bongaerts, P., T. Ridgway, E. Sampayo, and O. Hoegh-Guldberg, 2010: Assessing the 'deep reef refugia' hypothesis: focus on Caribbean reefs. Coral Reefs, 29(2), 309-327.

Boo, K.O., W.T. Kwon, and H.J. Baek, 2006: Change of extreme events of temperature and precipitation over Korea using regional projection of future climate change. Geophysical Research Letters, 33, L01701.

Brabson, B.B., D.H. Lister, P.D. Jones, and J.P. Palutikof, 2005: Soil moisture and predicted spells of extreme temperatures in Britain. Journal of Geophysical Research-Atmospheres, 110, D05104.

Branstator, G., and F. Selten, 2009: "Modes of Variability" and climate change. Journal of Climate, 22(10), 2639-2658.

Brayshaw, D.J., B. Hoskins, and M. Blackburn, 2008: The storm-track response to idealized SST perturbations in an aquaplanet GCM. Journal of the Atmospheric Sciences, 65(9), 2842-2860.

Brázdil, R., C. Pfister, H. Wanner, H. Von Storch, and J. Luterbacher, 2005: Historical climatology in Europe - The state of the art. Climatic Change, 70(3), 363-430.

Brázdil, R., Z.W. Kundzewicz, and G. Benito, 2006: Historical hydrology for studying flood risk in Europe. Hydrological Sciences Journal, 51(5), 739-764.

Brázdil, R., M. Trnka, P. Dobrovolny, K. Chromi, P. Hlavinka, and Z. Zalud, 2009: Variability of droughts in the Czech Republic, 1881-2006. Theoretical and Applied Climatology, 97(3-4), 297-315.

Breda, N., and V. Badeau, 2008: Forest tree responses to extreme drought and some biotic events: Towards a selection according to hazard tolerance? Comptes Rendus Geoscience, 340(9-10), 651-662.

Brockhaus, P., D. Luthi, and C. Schär, 2008: Aspects of the diurnal cycle in a regional climate model. Meteorologische Zeitschrift, 17(4), 433-443.
Bromirski, P.D., R.E. Flick, and D.R. Cayan, 2003: Storminess variability along the California coast: 1858-2000. Journal of Climate, 16(6), 982.

Brooks, H.E., and N. Dotzek, 2008: The spatial distribution of severe convective storms and an analysis of their secular changes. In: Climate Extremes and Society [Diaz, H.F., and R. Murnane (eds.)]. Cambridge University Press, Cambridge, NY, pp. 35-53.

Brovkin, V., T. Raddatz, C.H. Reick, M. Claussen, and V. Gayler, 2009: Global biogeophysical interactions between forest and climate. Geophysical Research Letters, 36, L07405.

Brown, J., M. Collins, and A. Tudhope, 2006: Coupled model simulations of midHolocene ENSO and comparisons with coral oxygen isotope record. Advances in Geosciences, 6, 29-33.

Brown, J.M., A.J. Souza, and J. Wolf, 2010: Surge modelling in the eastern Irish Sea: present and future storm impact. Ocean Dynamics, 60(2), 227-236.

Brown, R.D., and P.W. Mote, 2009: The response of Northern Hemisphere snow cover to a changing climate. Journal of Climate, 22(8), 2124-2145.

Brown, S.J., J. Caesar, and C.A.T. Ferro, 2008: Global changes in extreme daily temperature since 1950. Journal of Geophysical Research - Atmospheres, 113, D05115.

Brunet, M., P.D. Jones, J. Sigró, O. Saladié, E. Aguilar, A. Moberg, P.M. Della-Marta, D. Lister, A. Walther, and D. López, 2007: Temporal and spatial temperature variability and change over Spain during 1850-2005. Journal of Geophysical Research - Atmospheres, 112, D12117.

Bryan, K.R., P.S. Kench, and D.E. Hart, 2008: Multi-decadal coastal change in New Zealand: Evidence, mechanisms and implications. New Zealand Geographer, 64(2), 117-128

Büntgen, U., V. Trouet, D. Frank, H.H. Leuschner, D. Friedrichs, J. Luterbacher, and J. Esper, 2010: Tree-ring indicators of German summer drought over the last millennium. Quaternary Science Reviews, 29(7-8), 1005-1016.

Burke, E.J., and S.J. Brown, 2008: Evaluating uncertainties in the projection of future drought. Journal of Hydrometeorology, 9(2), 292-299.

Burke, E.J., S.J. Brown, and N. Christidis, 2006: Modeling the recent evolution of global drought and projections for the twenty-first century with the Hadley Centre climate model. Journal of Hydrometeorology, 7(5), 1113-1125.

Burke, E.J., R.H.J. Perry, and S.J. Brown, 2010: An extreme value analysis of UK drought and projections of change in the future. Journal of Hydrology, 388(1-2), 131-143.

Burkholder, B.A. and D.J. Karoly, 2007: An assessment of US climate variability using the Climate Extremes Index. In: Proceedings of the Nineteenth Conference on Climate Variability and Change, San Antonio, TX, 15-18 January 2007, pp. 2B.9.

Burton, A., C.G. Kilsby, H.J. Fowler, P.S.P. Cowpertwait, and P.E. O'Connell, 2008: RainSim: A spatial-temporal stochastic rainfall modelling system. Environmental Modelling \& Software, 23(12), 1356-1369.

Caesar, J., L. Alexander, and R. Vose, 2006: Large-scale changes in observed daily maximum and minimum temperatures: Creation and analysis of a new gridded data set. Journal of Geophysical Research - Atmospheres, 111, D05101.

Caesar, J., L.V. Alexander, B. Trewin, K. Tse-ring, L. Sorany, V. Vuniyayawa, N. Keosavang, A. Shimana, M.M. Htay, J. Karmacharya, D.A. Jayasinghearachchi, J. Sakkamart, A. Soares, L.T. Hung, L.T. Thuong, C.T. Hue, N.T.T. Dung, P.V. Hung, H.D. Cuong, N.M. Cuong, and S. Sirabaha, 2011: Changes in temperature and precipitation extremes over the Indo-Pacific region from 1971 to 2005. International Journal of Climatology, 31(6), 791-801.

Callaghan, J., and S. Power, 2011: Variability and decline in the number of severe tropical cyclones making land-fall over eastern Australia since the late nineteenth century. Climate Dynamics, 37(3-4), 647-662, doi:10.1007/s0038200010-00883-00382.

Camberlin, P., V. Moron, R. Okoola, N. Philippon, and W. Gitau, 2009: Components of rainy seasons' variability in Equatorial East Africa: onset, cessation, rainfall frequency and intensity. Theoretical and Applied Climatology, 98(3-4), 237-249.

Cambers, G., 2009: Caribbean beach changes and climate change adaptation. Aquatic Ecosystem Health \& Management, 12(2), 168-176. 
Cameron, D., 2006: An application of the UKCIP02 climate change scenarios to flood estimation by continuous simulation for a gauged catchment in the northeast of Scotland, UK (with uncertainty). Journal of Hydrology, 328(1-2), 212-226.

Camilloni, I.A. and V.R. Barros, 2003: Extreme discharge events in the Paraná River and their climate forcing. Journal of Hydrology, 278(1-4), 94-106.

Campbell, J.D., M.A. Taylor, T.S. Stephenson, R.A. Watson, and F.S. Whyte, 2011: Future climate of the Caribbean from a regional climate model. International Journal of Climatology, 31(12), 1866-1878, doi:10.1002/joc.2200.

Camuffo, D., C. Bertolin, M. Barriendos, F. Dominguez-Castro, C. Cocheo, S. Enzi, M. Sghedoni, A. della Valle, E. Garnier, M.J. Alcoforado, E. Xoplaki, J. Luterbacher, N. Diodato, M. Maugeri, M.F. Nunes, and R. Rodriguez, 2010: 500-year temperature reconstruction in the Mediterranean Basin by means of documentary data and instrumental observations. Climatic Change, 101(1-2), 169-199.

Cane, M.A., 2005: The evolution of El Niño, past and future. Earth and Planetary Science Letters, 230(3-4), 227-240.

Cao, Z., 2008: Severe hail frequency over Ontario, Canada: Recent trend and variability. Geophysical Research Letters, 35, L14803.

Caplan-Auerbach, J., and C. Huggel, 2007: Precursory seismicity associated with frequent, large ice avalanches on Iliamna volcano, Alaska, USA. Journal of Glaciology, 53(180), 128-140.

Capra, L., 2006: Abrupt climatic changes as triggering mechanisms of massive volcanic collapses. Journal of Volcanology and Geothermal Research, 155(3-4), 329-333.

Carey, M., 2005: Living and dying with glaciers: people's historical vulnerability to avalanches and outburst floods in Peru. Global and Planetary Change, 47(2-4), 122-134.

Carey-Smith, T., S. Dean, J. Vial, and C. Thompson, 2010: Changes in precipitation extremes for New Zealand: climate model predictions. Weather and Climate, 30, 23-48.

Carter, T.R., R.N. Jones, X. Lu, S. Bhadwal, C. Conde, L.O. Mearns, B.C. O'Neill, M.D.A. Rounsevell, and M.B. Zurek, 2007: New assessment methods and the characterisation of future conditions. In: Climate Change 2007. Impacts, Adaptation and Vulnerability. Contribution of Working Group II to the Fourth Assessment Report of the Intergovernmental Panel on Climate Change [Parry, M.L., O.F. Canziani, J.P. Palutikof, P.J. Van Der Linde, and C.E. Hanson (eds.)]. Cambridge University Press, Cambridge, UK, pp. 133-171.

Cassou, C., L. Terray, and A.S. Phillips, 2005: Tropical Atlantic influence on European heat waves. Journal of Climate, 18(15), 2805-2811.

Catto, J.L., L.C. Shaffrey, and K.I. Hodges, 2010: Can climate models capture the structure of extratropical cyclones? Journal of Climate, 23(7), 1621-1635.

Cavazos, T., C. Turrent, and D.P. Lettenmaier, 2008: Extreme precipitation trends associated with tropical cyclones in the core of the North American monsoon. Geophysical Research Letters, 35, L21703.

Cayan, D.R., P.D. Bromirski, K. Hayhoe, M. Tyree, M.D. Dettinger, and R.E. Flick, 2008: Climate change projections of sea level extremes along the California coast. Climatic Change, 87(S1), 57-73.

Cayan, D.R., T. Das, D.W. Pierce, T.P. Barnett, M. Tyree, and A. Gershunov, 2010: Future dryness in the southwest US and the hydrology of the early 21 st century drought. Proceedings of the National Academy of Sciences, 107(50), 21271-21276.

Cazenave, A. and W. Llovel, 2010: Contemporary sea level rise. Annual Review of Marine Science, 2, 145-173.

CCSP, 2008: Weather and Climate Extremes in a Changing Climate. Regions of Focus: North America, Hawaii, Caribbean, and U.S. Pacific Islands. A Report by the U.S. Climate Change Science Program and the Subcommittee on Global Change Research [Karl, T.R., G.A. Meehl, D.M. Christopher, S.J. Hassol, A.M. Waple, and W.L. Murray (eds.)]. Department of Commerce, NOAA's National Climatic Data Center, Washington, DC, $164 \mathrm{pp}$

Chambers, L.E. and G.M. Griffiths, 2008: The changing nature of temperature extremes in Australia and New Zealand. Australian Meteorological Magazine, 57(1), 13-35.

Chan, J.C.L. and M. Xu, 2009: Inter-annual and inter-decadal variations of landfalling tropical cyclones in East Asia. Part I: Time series analysis. International Journal of Climatology, 29(9), 1285-1293.
Chang, E.K.M., 2007: Assessing the Increasing Trend in Northern Hemisphere Winter Storm Track Activity Using Surface Ship Observations and a Statistical Storm Track Model. Journal of Climate, 20, 5607-5628.

Chang, E.K.M., 2009: Are band-pass variance statistics useful measures of storm track activity? Re-examining storm track variability associated with the NAO using multiple storm track measures. Climate Dynamics, 33(2-3), 277-296.

Chang, E.K.M. and Y. Guo, 2007: Is the number of North Atlantic tropical cyclones significantly underestimated prior to the availability of satellite observations? Geophysical Research Letters, 34, L14801.

Chauvin, F., J.F. Royer, and M. Deque, 2006: Response of hurricane-type vortices to global warming as simulated by ARPEGE-Climat at high resolution. Climate Dynamics, 27(4), 377-399.

Chen, C.-T. and T. Knutson, 2008: On the verification and comparison of extreme rainfall indices from climate models. Journal of Climate, 21(7), 1605-1621.

Chen, J., F.P. Brissette, and R. Leconte, 2011: Uncertainty of downscaling method in quantifying the impact of climate change on hydrology. Journal of Hydrology, 401(3-4), 190-202.

Chen, J.L., C.R. Wilson, B.D. Tapley, Z.L. Yang, and G.Y. Niu, 2009: 2005 drought event in the Amazon River basin as measured by GRACE and estimated by climate models. Journal of Geophysical Research - Solid Earth, 114, B05404.

Chenoweth, M. and M. Devine, 2008: A document-based 318-year record of tropical cyclones in the Lesser Antilles, 1690-2007. Geochemistry Geophysics Geosystems, 9, Q08013.

Cherchi, A., S. Masina, and A. Navarra, 2008: Impact of extreme $\mathrm{CO}_{2}$ levels on tropical climate: a CGCM study. Climate Dynamics, 31(7-8), 743-758.

Cherry, N.J., 1988: Comment on long-term associations between wind speeds and the urban heat-island of Phoenix, Arizona. Journal of Applied Meteorology, 27(7), 878-880

Chiapello, I., C. Moulin, and J.M. Prospero, 2005: Understanding the long-term variability of African dust transport across the Atlantic as recorded in both Barbados surface concentrations and large-scale Total Ozone Mapping Spectrometer (TOMS) optical thickness. Journal of Geophysical Research Atmospheres, 110, D18S10.

Chiarle, M., S. lannotti, G. Mortara, and P. Deline, 2007: Recent debris flow occurrences associated with glaciers in the Alps. Global and Planetary Change, 56(1-2), 123-136.

Chini, N., P. Stansby, J. Leake, J. Wolf, J. Roberts-Jones, and J. Lowe, 2010: The impact of sea level rise and climate change on inshore wave climate: A case study for East Anglia (UK). Coastal Engineering, 57(11-12), 973-984.

Choi, G., D. Collins, R. Guoyu, B. Trewin, M. Baldi, Y. Fukuda, M. Afzaal, T. Pianmana, P. Gomboluudev, P.T.T. Huong, N. Lias, W.T. Kwon, K.O. Boo, Y.M. Cha, and Y. Zhou, 2009: Changes in means and extreme events of temperature and precipitation in the Asia-Pacific Network region, 1955-2007. International Journal of Climatology, 29(13), 1956-1975.

Chowdhury, M.R., P.S. Chu, and T. Schroeder, 2007: ENSO and seasonal sea-level variability - A diagnostic discussion for the US-affiliated Pacific Islands. Theoretical and Applied Climatology, 88(3-4), 213-224

Christensen, J.H. and O.B. Christensen, 2003: Climate modeling: Severe summertime flooding in Europe. Nature, 421, 805-806.

Christensen, J.H. and O.B. Christensen, 2007: A summary of the PRUDENCE model projections of changes in European climate by the end of this century. Climatic Change, 81(S1), 7-30.

Christensen, J.H., B. Hewitson, A. Busuioc, A. Chen, X. Gau, I. Held, R. Jones, R. Kolli, W. Kwon, R. Laprise, V. Magaña Rueda, L. Mearns, C. Menéndez, J. Räisänen, A. Rinke, A. Sarr, and P. Whetton, 2007: Regional climate projections. In: Climate Change 2007: The Physical Science Basis. Contribution of Working Group I to the Fourth Assessment Report of the Intergovernmental Panel on Climate Change [Solomon, S., D. Qin, M. Manning, Z. Chen, M. Marquis, K.B. Averyt, M. Tignor and H.L. Miller (eds.)]. Cambridge University Press, Cambridge, UK, pp 847-940.

Christidis, N., P.A. Stott, S. Brown, G. Hegerl, and J. Caesar, 2005: Detection of changes in temperature extremes during the second half of the 20th century. Geophysical Research Letters, 32, L20716. 
Christidis, N., P.A. Stott, G.S. Jones, H. Shiogama, T. Nozawa, and J. Luterbacher, 2011a: Human activity and anomalously warm seasons in Europe. International Journal of Climatology, doi:10.1002/joc.2262.

Christidis, N., P.A. Stott, and S.J. Brown, 2011b: The role of human activity in the recent warming of extremely warm daytime temperatures. Journal of Climate, 24(7), 1922-1930.

Church, J.A. and N.J. White, 2011: Sea-level rise from the late 19th to the early 21st century. Surveys in Geophysics, 32(4-5), 585-602, doi:10.1007/s10712-1001119119-10711.

Church, J.A., J.R. Hunter, K.L. McInnes, and N.J. White, 2006a: Sea-level rise around the Australian coastline and the changing frequency of extreme sea-level events. Australian Meteorological Magazine, 55(4), 253-260.

Church, J.A., N.J. White, and J.R. Hunter, 2006b: Sea-level rise at tropical Pacific and Indian Ocean Islands. Global and Planetary Change, 53(3), 155-168.

Church, J.A., N.J. White, J.R. Hunter, K.L. McInnes, P. J. Cowell , and S.P. O'Farrell, 2008: Sea-level rise. In: Transitions: Pathways Towards Sustainable Urban Development in Australia [Newton, E.P. (ed.)]. CSIRO Publishing and Springer Science, Collingwood, Australia, pp. 691.

Church, J.A., D. Roemmich, C.M. Domingues, J.K. Willis, N.J. White, J.E. Gilson, D. Stammer, A. Köhl, D.P. Chambers, F.W. Landerer, J. Marotzke, J.M. Gregory, T. Suzuki, A. Cazenave, and P.-Y. Le Traon, 2010: Ocean temperature and salinity contributions to global and regional sea-level change. In: Understanding SeaLevel Rise and Variability [Church, J.A., P.L. Woodworth, T. Aarup, and W.S. Wilson (eds.)]. Wiley-Blackwell, Chichester, UK, pp. 143-176.

Church, J.A., J.M. Gregory, N.J. White, S.M. Platten, and J.X. Mitrovica, 2011: Understanding and projecting sea level change. Oceanography, 24(2), 130-143.

Chust, G., A. Borja, P. Liria, I. Galparsoro, M. Marcos, A. Caballero, and R. Castro, 2009: Human impacts overwhelm the effects of sea-level rise on Basque coastal habitats (N Spain) between 1954 and 2004. Estuarine Coastal and Shelf Science, 84(4), 453-462.

Ciais, P., M. Reichstein, N. Viovy, A. Granier, J. Ogee, V. Allard, M. Aubinet, N. Buchmann, C. Bernhofer, A. Carrara, F. Chevallier, N. De Noblet, A. Friend, P. Friedlingstein, T. Grunwald, B. Heinesch, P. Keronen, A. Knohl, G. Krinner, D. Loustau, G. Manca, G. Matteucci, F. Miglietta, J. Ourcival, D. Papale, K. Pilegaard, S. Rambal, G. Seufert, J. Soussana, M. Sanz, E. Schulze, T. Vesala, and R. Valentini, 2005: Europe-wide reduction in primary productivity caused by the heat and drought in 2003. Nature, 437(7058), 529-533.

Clague, J.J. and S.G. Evans, 2000: A review of catastrophic drainage of morainedammed lakes in British Columbia. Quaternary Science Reviews, 19(17-18), 1763-1783.

Clark, R.T., S.J. Brown, and J.M. Murphy, 2006: Modeling northern hemisphere summer heat extreme changes and their uncertainties using a physics ensemble of climate sensitivity experiments. Journal of Climate, 19(17), 4418-4435.

Clark, R.T., J.M. Murphy, and S.J. Brown, 2010: Do global warming targets limit heatwave risk? Geophysical Research Letters, 37, L17703.

Clarke, G.K.C., 1982: Glacier outburst floods from 'Hazard Lake,' Yukon Territory, and the problem of flood magnitude prediction. Journal of Glaciology, 28(98), 3-21.

Clarke, M.L., and H.M. Rendell, 2009: The impact of North Atlantic storminess on western European coasts: A review. Quaternary International, 195, 31-41.

Clemmensen, L.B., M. Bjornsen, A. Murray, and K. Pedersen, 2007: Formation of aeolian dunes on Anholt, Denmark since AD 1560: A record of deforestation and increased storminess. Sedimentary Geology, 199(3-4), 171-187.

Clow, D.W., 2010: Changes in the timing of snowmelt and streamflow in Colorado: a response to recent warming. Journal of Climate, 23(9), 2293-2306.

Cobb, K.M., C.D. Charles, H. Cheng, and R.L. Edwards, 2003: El Niño/Southern Oscillation and tropical Pacific climate during the last millennium. Nature, 424, 271-276.

Coelho, C., R. Silva, F. Veloso-Gomes, and F. Taveira-Pinto, 2009: Potential effects of climate change on northwest Portuguese coastal zones. ICES Journal of Marine Science, 66(7), 1497-1507.

Coles, S., 2001: An Introduction to Statistical Modeling of Extreme Values. SpringerVerlag, Heidelberg, Germany, 208 pp.
Collini, E.A., E.H. Berbery, V. Barros, and M. Pyle, 2008: How does soil moisture influence the early stages of the South American monsoon? Journal of Climate, 21(2), 195-213.

Collins, M., B.B.B. Booth, G.R. Harris, J.M. Murphy, D.M.H. Sexton, and M.J. Webb, 2006: Towards quantifying uncertainty in transient climate change. Climate Dynamics, 27(2-3), 127-147.

Collins, M., S.I. An, W.J. Cai, A. Ganachaud, E. Guilyardi, F.F. Jin, M. Jochum, M. Lengaigne, S. Power, A. Timmermann, G. Vecchi, and A. Wittenberg, 2010: The impact of global warming on the tropical Pacific ocean and El Nino. Nature Geoscience, 3(6), 391-397.

Compo, G.P., J.S. Whitaker, and P.D. Sardeshmukh, 2006: Feasibility of a 100-year reanalysis using only surface pressure data. Bulletin of the American Meteorological Society, 87(2), 175-190.

Conroy, J.L., A. Restrepo, J.T. Overpeck, M. Seinitz-Kannan, J.E. Cole, M.B. Bush, and P.A. Colinvaux, 2009: Unprecedented recent warming of surface temperatures in the eastern tropical Pacific Ocean. Nature Geoscience, 2(1), 46-50.

Conway, D., C.E. Hanson, R. Doherty, and A. Persechino, 2007: GCM simulations of the Indian Ocean dipole influence on East African rainfall: Present and future. Geophysical Research Letters, 34, L03705.

Conway, D., A. Persechino, S. Ardoin-Bardin, H. Hamandawana, C. Dieulin, and G. Mahe, 2009: Rainfall and water resources variability in sub-Saharan Africa during the twentieth century. Journal of Hydrometeorology, 10(1), 41-59.

Cook, B.I., G.B. Bonan, and S. Levis, 2006: Soil moisture feedbacks to precipitation in southern Africa. Journal of Climate, 19(17), 4198-4206.

Cook, K.H. and E.K. Vizy, 2006: Coupled model simulations of the west African monsoon system: Twentieth- and twenty-first-century simulations. Journal of Climate, 19(15), 3681-3703.

Coppola, E. and F. Giorgi, 2005: Climate change in tropical regions from highresolution time-slice AGCM experiments. Quarterly Journal of the Royal Meteorological Society, 131(612), 3123-3145.

Cordero, E.C. and P.M.D. Forster, 2006: Stratospheric variability and trends in models used for the IPCC AR4. Atmospheric Chemistry and Physics, 6(12), 5369-5380.

Corti, T., V. Muccione, P. Köllner-Heck, D. Bresch, and S.I. Seneviratne, 2009: Simulating past droughts and associated building damages in France. Hydrology and Earth System Sciences, 13(9), 1739-1747.

Costa, A.C., and A. Soares, 2009: Trends in extreme precipitation indices derived from a daily rainfall database for the South of Portugal. International Journal of Climatology, 29(13), 1956-1975.

CSIRO, 2007: Climate Change in Australia. CSIRO, Australia, 148 pp.

Cubasch, U., J. Waszkewitz, G. Hegerl, and J. Perlwitz, 1995: Regional climate changes as simulated in time-slice experiments. Climatic Change, 31(2-4), 273-304.

Cullen, N.J., T. Molg, G. Kaser, K. Hussein, K. Steffen, and D.R. Hardy, 2006: Kilimanjaro Glaciers: Recent areal extent from satellite data and new interpretation of observed 20th century retreat rates. Geophysical Research Letters, 33, L16502.

Cunderlik, J.M., and T.B.M.J. Ouarda, 2009: Trends in the timing and magnitude of floods in Canada. Journal of Hydrology, 375(3-4), 471-480.

Cunningham, P., and M.J. Reeder, 2009: Severe convective storms initiated by intense wildfires: Numerical simulations of pyro-convection and pyro-tornadogenesis. Geophysical Research Letters, 36, L12812.

da Silva, A.E. and L.M.V. de Carvalho, 2007: Large-scale index for South America Monsoon (LISAM). Atmospheric Science Letters, 8(2), 51-57.

Dai, A., T.T. Qian, K.E. Trenberth, and J.D. Milliman, 2009: Changes in continental freshwater discharge from 1948 to 2004. Journal of Climate, 22(10), 2773-2792

Dai, A., 2011: Drought under global warming: a review. Wiley Interdisciplinary Reviews: Climate Change, 2(1), 45-65.

Dai, A.G., K.E. Trenberth, and T.T. Qian, 2004: A global dataset of Palmer Drought Severity Index for 1870-2002: Relationship with soil moisture and effects of surface warming. Journal of Hydrometeorology, 5(6), 1117-1130.

Dairaku, K., S. Emori, and H. Higashi, 2008: Potential changes in extreme events under global climate change. Journal of Disaster Research, 3(1), 39-50. 
Danard, M.B., S.K. Dube, G. Gönnert, A. Munroe, T.S. Murty, P. Chittibabu, A.D. Rao, and P.C. Sinha, 2004: Storm surges from extra-tropical cyclones. Natural Hazards, 32(2), 177-190.

Dankers, R., O.B. Christensen, L. Feyen, M. Kalas, and A. de Roo, 2007: Evaluation of very high-resolution climate model data for simulating flood hazards in the Upper Danube Basin. Journal of Hydrology, 347(3-4), 319-331.

Dankers, R. and L. Feyen, 2008: Climate change impact on flood hazard in Europe: An assessment based on high-resolution climate simulations. Journal of Geophysical Research - Atmospheres, 113, D19105.

Dankers, R. and L. Feyen, 2009: Flood hazard in Europe in an ensemble of regional climate scenarios. Journal of Geophysical Research - Atmospheres, 114, D16108.

Dasgupta, S., B. Laplante, C. Meisner, D. Wheeler, and J. Yan, 2009: The impact of sea level rise on developing countries: a comparative analysis. Climatic Change, 93(3-4), 379-388.

Dawson, J.L., and S.G. Smithers, 2010: Shoreline and beach volume change between 1967 and 2007 at Raine Island, Great Barrier Reef, Australia. Global and Planetary Change, 72(3), 141-154.

de Elía, R., D. Caya, H. Côté, A. Frigon, S. Biner, M. Giguère, D. Paquin, R. Harvey, and D. Plummer, 2008: Evaluation of uncertainties in the CRCM-simulated North American climate. Climate Dynamics, 30(2-3), 113-132.

de Jong, R., S. Bjorck, L. Bjorkman, and L.B. Clemmensen, 2006: Storminess variation during the last 6500 years as reconstructed from an ombrotrophic peat bog in Halland, southwest Sweden. Journal of Quaternary Science, 21(8), 905-919.

de Jong, R., K. Schoning, and S. Bjorck, 2007: Increased aeolian activity during humidity shifts as recorded in a raised bog in south-west Sweden during the past 1700 years. Climate of the Past, 3(3), 411-422.

Dean, S.M. and P.A. Stott, 2009: The effect of local circulation variability on the detection and attribution of New Zealand temperature trends. Journal of Climate, 22(23), 6217-6229.

Debernard, J.B. and L.P. Roed, 2008: Future wind, wave and storm surge climate in the Northern Seas: a revisit. Tellus Series A - Dynamic Meteorology and Oceanography, 60(3), 427-438.

Deeming, K.R., B. McGuire, and P. Harrop, 2010: Climate forcing of volcano lateral collapse: evidence from Mount Etna, Sicily. Philosophical Transactions of the Royal Society A: Mathematical, Physical and Engineering Sciences, 368(1919), 2559-2577.

Defeo, O., A. McLachlan, D.S. Schoeman, T.A. Schlacher, J. Dugan, A. Jones, M. Lastra, and F. Scapini, 2009: Threats to sandy beach ecosystems: A review. Estuarine Coastal and Shelf Science, 81(1), 1-12.

DeGaetano, A.T., 2009: Time-dependent changes in extreme-precipitation returnperiod amounts in the Continental United States. Journal of Applied Meteorology and Climatology, 48, 2086-2099.

Delaloye, R., T. Strozzi, C. Lambiel, E. Perruchoud, and H. Raetzo, 2008: Landslide-like development of rockglaciers detected with ERS-1/2 SAR interferometry. In: Proceedings of the FRINGE 2007 Symposium at ESRIN [Lacoste, H., and L. Ouwehand (eds.)], Frascati, Italy, 26-30 November 2007, ESA Communication Production Office, ESTEC, Noordwijk, The Netherlands, pp. 1-6.

Delgado, J.M., H. Apel, and B. Merz, 2009: Flood trends and variability in the Mekong River. Hydrology and Earth System Sciences, 6(3), 6691-6719.

Della-Marta, P.M. and H. Wanner, 2006: A method of homogenizing the extremes and mean of daily temperature measurements. Journal of Climate, 19, 4179-4197.

Della-Marta, P.M., M.R. Haylock, J. Luterbacher, and H. Wanner, 2007a: Doubled length of western European summer heat waves since 1880. Journal of Geophysical Research - Atmospheres, 112, D15103.

Della-Marta, P.M., J. Luterbacher, H. von Weissenfluh, E. Xoplaki, M. Brunet, and H. Wanner, 2007b: Summer heat waves over western Europe 1880-2003, their relationship to large-scale forcings and predictability. Climate Dynamics, 29(2-3), 251-275.

Della-Marta, P.M., H. Mathis, C. Frei, M.A. Liniger, J. Kleinn, and C. Appenzeller, 2009: The return period of wind storms over Europe. International Journal of Climatology, 29(3), 437-459.
DeMaria, M., J.A. Knaff, and B.H. Connell, 2001: A tropical cyclone genesis parameter for the tropical Atlantic. Weather and Forecasting, 16(2), 219-233.

Deo, R.C., J.I. Syktus, C.A. McAlpine, P.J. Lawrence, H.A. McGowan, and S.R. Phinn, 2009: Impact of historical land cover change on daily indices of climate extremes including droughts in eastern Australia. Geophysical Research Letters, 36, L08705.

Department of Climate Change, 2009: Climate Change Risks to Australia's Coast. A First Pass National Assessment. Report published by the Department of Climate Change, Australian Government, Canberra, Australia, 172 pp.

Déqué, M., S. Somot, E. Sanchez-Gomez, C. Goodess, D. Jacob, G. Lenderink, and 0. Christensen, 2011: The spread amongst ENSEMBLES regional scenarios: regional climate models, driving general circulation models and interannual variability. Climate Dynamics, doi:10.1007/s00382-00011-01053-x.

Deser, C., R.A. Tomas, and S. Peng, 2007: The transient atmospheric circulation response to North Atlantic SST and sea ice anomalies. Journal of Climate, 20(18), 4751-4767.

Di Baldassarre, G., A. Montanari, H. Lins, D. Koutsoyiannis, L. Brandimarte, and G. Blöschl, 2010: Flood fatalities in Africa: From diagnosis to mitigation. Geophysical Research Letters, 37, L22402.

Dibike, Y.B., P. Gachon, A. St-Hilaire, T. Ouarda, and V.T.V. Nguyen, 2008: Uncertainty analysis of statistically downscaled temperature and precipitation regimes in Northern Canada. Theoretical and Applied Climatology, 91(1-4), 149-170.

Diffenbaugh, N.S. and M. Ashfaq, 2010: Intensification of hot extremes in the United States. Geophysical Research Letters, 37(15), L15701.

Diffenbaugh, N.S., J.S. Pal, F. Giorgi, and X. Gao, 2007: Heat stress intensification in the Mediterranean climate change hotspot. Geophysical Research Letters, 34 L11706.

Diffenbaugh, N.S., R.J. Trapp, and H. Brooks, 2008: Does global warming influence tornado activity? Eos Transactions (AGU), 89(53), 553.

Ding, T., W. Qian, and Z. Yan, 2010: Changes in hot days and heat waves in China during 1961-2007. International Journal of Climatology, 30(10), 1452-1462.

Dirmeyer, P.A., R.D. Koster, and Z. Guo, 2006: Do global models properly represent the feedback between land and atmosphere? Journal of Hydrometeorology, 7(6), 1177-1198.

Dobrovolny, P., A. Moberg, R. Brazdil, C. Pfister, R. Glaser, R. Wilson, A. van Engelen, D. Limanowka, A. Kiss, M. Halickova, J. Mackova, D. Riemann, J. Luterbacher, and R. Bohm, 2010: Monthly, seasonal and annual temperature reconstructions for Central Europe derived from documentary evidence and instrumental records since AD 1500. Climatic Change, 101(1-2), 69-107.

Dodet, G., X. Bertin, and R. Taborda, 2010: Wave climate variability in the North-East Atlantic Ocean over the last six decades. Ocean Modelling, 31(3-4), 120-131.

Dole, R., M. Hoerling, J. Perlwitz, J. Eischeid, P. Pegion, T. Zhang, X.-W. Quan, T. Xu, and D. Murray, 2011: Was there a basis for anticipating the 2010 Russian heat wave? Geophysical Research Letters, 38, L06702.

Donat, M.G., G.C. Leckebusch, J.G. Pinto, and U. Ulbrich, 2010a: European storminess and associated circulation weather types: future changes deduced from a multimodel ensemble of GCM simulations. Climate Research, 42(1), 27-43

Donat, M.G., G.C. Leckebusch, J.G. Pinto, and U. Ulbrich, 2010b: Examination of wind storms over Central Europe with respect to circulation weather types and NAO phases. International Journal of Climatology, 30(9), 1289-1300.

Donat, M.G., G.C. Leckebusch, S. Wild, and U. Ulbrich, 2011: Future changes of European winter storm losses and extreme wind speeds in multi-model GCM and RCM simulations. Natural Hazards and Earth System Sciences, 11(5), 1351-1370.

Dong, B., R. Sutton, and T. Woollings, 2011: Changes of interannual NAO variability in response to greenhouse gases forcing. Climate Dynamics, 37(7-8), 1621-1641, doi:10.1007/s00382-00010-00936-00386.

Donnelly, J.P., and J.D. Woodruff, 2007: Intense hurricane activity over the past 5,000 years controlled by El Niño and the West African monsoon. Nature, 447, 465-468.

D'Onofrio, A., J.P. Boulanger, and E.C. Segura, 2010: CHAC: a weather pattern classification system for regional climate downscaling of daily precipitation. Climatic Change, 98(3-4), 405-427. 
Donohue, R.J., T.R. McVicar, and M.L. Roderick, 2010: Assessing the ability of potential evaporation formulations to capture the dynamics in evaporative demand within a changing climate. Journal of Hydrology, 386(1-4), 186-197.

Doser, D.I., K.R. Wiest, and J. Sauber, 2007: Seismicity of the Bering Glacier region and its relation to tectonic and glacial processes. Tectonophysics, 439(1-4), 119-127.

Douglas, E.M., R.M. Vogel, and C.N. Kroll, 2000: Trends in floods and low flows in the United States: impact of spatial correlation. Journal of Hydrology, 240(1-2), 90-105.

Dragani, W.C., P.B. Martin, C.G. Simionato, and M.I. Campos, 2010: Are wind wave heights increasing in south-eastern south American continental shelf between $32^{\circ} \mathrm{S}$ and $40^{\circ} \mathrm{S}$ ? Continental Shelf Research, 30(5), 481-490.

Dufek, A.S. and T. Ambrizzi, 2008: Precipitation variability in Sao Paulo State, Brazil. Theoretical and Applied Climatology, 93(3-4), 167-178.

Dufek, A.S., T. Ambrizzi, and R.P. da Rocha, 2008: Are reanalysis data useful for calculating climate indices over South America? In: Trends and Directions in Climate Research [Gimeno, L., R. GarciaHerrera, and R.M. Trigo (eds.)]. Vol. 1146, Annals of the New York Academy of Sciences, New York, NY, USA, pp. 87-104.

Durante, F., and G. Salvadori, 2010: On the construction of multivariate extreme value models via copulas. Environmetrics, 21(2), 143-161.

Durão, R.M., M.J. Pereira, A.C. Costa, J. Delgado, G. del Barrio, and A. Soares, 2010: Spatial-temporal dynamics of precipitation extremes in southern Portugal: a geostatistical assessment study. International Journal of Climatology, 30(10), 1526-1537.

Durre, I., J.M. Wallace, and D.P. Lettenmaier, 2000: Dependence of extreme daily maximum temperatures on antecedent soil moisture in the contiguous United States during summer. Journal of Climate, 13(14), 2641-2651.

Durre, I., M.J. Menne, and R.S. Vose, 2008: Strategies for evaluating quality assurance procedures. Journal of Applied Meteorology and Climatology, 47(6), 1785-1791.

Dussaillant, A., G. Benito, W. Buytaert, P. Carling, C. Meier, and F. Espinoza, 2010: Repeated glacial-lake outburst floods in Patagonia: an increasing hazard? Natural Hazards, 54(2), 469-481.

Dutton, J.F., C.J. Poulsen, and J.L. Evans, 2000: The effect of global climate change on the regions of tropical convection in CSM1. Geophysical Research Letters, 27(19), 3049-3052.

Easterling, D.R., D.M. Anderson, S.J. Cohen, W.J. Gutowski, G.J. Holland, K.E. Kunkel, T.C. Peterson, R.S. Pulwarty, R.J. Stouffer, and M.F. Wehner, 2008: Measures to improve our understanding of weather and climate extremes. In: Weather and Climate Extremes in a Changing Climate. Regions of focus: North America, Hawaii, Caribbean, and U.S. Pacific Islands. [Karl, T.R., G.A. Meehl, D.M. Christopher, S.J. Hassol, A.M. Waple, and W.L. Murray (eds.)]. A Report by the U.S. Climate Change Science Program and the Subcommittee on Global Change Research, Washington, DC, pp. 117-126.

Eichler, T., and W. Higgins, 2006: Climatology and ENSO-related variability of North American extratropical cyclone activity. Journal of Climate, 19(10), 2076-2093.

Elsner, J.B., J.P. Kossin, and T.H. Jagger, 2008: The increasing intensity of the strongest tropical cyclones. Nature, 455(7209), 92-95.

Eltahir, E.A.B. and P.A.J.F. Yeh, 1999: On the asymmetric response of aquifer water level to floods and droughts in Illinois. Water Resources Research, 35(4), 1199-1217.

Ely, L.L., Y. Enzel, V.R. Baker, and D.R. Cayan, 1993: A 5000-year record of extreme floods and climate-change in the southwestern United-States. Science, 262(5132), 410-412.

Emanuel, K.A., 1987: Dependence of hurricane intensity on climate. Nature, 326(61112), 483-485.

Emanuel, K.A., 2000: A statistical analysis of tropical cyclone intensity. Monthly Weather Review, 128(4), 1139-1152.

Emanuel, K.A., 2007: Environmental factors affecting tropical cyclone power dissipation. Journal of Climate, 20(22), 5497-5509.

Emanuel, K.A., 2010: Tropical cyclone activity downscaled from NOAA-CIRES reanalysis, 1908-1958. Journal of Advances in Modeling Earth Systems, 2, 1, doi:10.3894/JAMES.2010.2.1.
Emanuel, K.A., R. Sundararajan, and J. Williams, 2008: Hurricanes and global warming: Results from downscaling IPCC AR4 simulations. Bulletin of the American Meteorological Society, 89(3), 347-367.

Emori, S. and S.J. Brown, 2005: Dynamic and thermodynamic changes in mean and extreme precipitation under changed climate. Geophysical Research Letters, 32, L17706.

Engelstaedter, S., I. Tegen, and R. Washington, 2006: North African dust emissions and transport. Earth-Science Reviews, 79(1-2), 73-100.

Essery, R., N. Rutter, J. Pomeroy, R. Baxter, M. Stahli, D. Gustafsson, A. Barr, P. Bartlett, and K. Elder, 2009: An evaluation of forest snow process simulations. Bulletin of the American Meteorological Society, 90(8), 1120-1135.

Evan, A.T., D.J. Vimont, A.K. Heidinger, J.P. Kossin, and R. Bennartz, 2009: The role of aerosols in the evolution of tropical North Atlantic Ocean temperature anomalies. Science, 324(5928), 778-781.

Evans, S.G., and J.J. Clague, 1998: Rock avalanche from Mount Munday, Waddington Range, British Columbia, Canada. Landslide News, 11, 23-25.

Favre, A., and A. Gershunov, 2006: Extra-tropical cyclonic/anticyclonic activity in North-Eastern Pacific and air temperature extremes in Western North America. Climate Dynamics, 26(6), 617-629.

Favre, A., and A. Gershunov, 2009: North Pacific cyclonic and anticyclonic transients in a global warming context: possible consequences for Western North American daily precipitation and temperature extremes. Climate Dynamics, 32(7-8), 969-987.

Fedorov, A. and P. Konstantinov, 2003: Observations of surface dynamics with thermokarst initiation, Yukechi site, central Yukutia. In: Proceedings of the 8th International Conference on Permafrost [Philips, M., S.M. Springman, and L.U. Arenson (eds.)]. Zurich, Switzerland, 21-25 July 2003, Balkema, Netherlands, pp. 239-243.

Fekete, B.M., C.J. Vörösmarty, J.O. Roads, and C.J. Willmott, 2004: Uncertainties in precipitation and their impacts on runoff estimates. Journal of Climate, 17(2), 294-304.

Feyen, L., and R. Dankers, 2009: Impact of global warming on streamflow drought in Europe. Journal of Geophysical Research - Atmospheres, 114, D17116.

Findell, K.L. and T.L. Delworth, 2005: A modeling study of dynamic and thermodynamic mechanisms for summer drying in response to global warming. Geophysical Research Letters, 32, L16702.

Findell, K.L. and E.A.B. Eltahir, 2003a: Atmospheric controls on soil moisture-boundary layer interactions. Part I: Framework development. Journal of Hydrometeorology, 4(3), 552-569.

Findell, K.L. and E.A.B. Eltahir, 2003b: Atmospheric controls on soil moisture-boundary layer interactions. Part II: Feedbacks within the continental United States. Journal of Hydrometeorology, 4(3), 570-583.

Finnis, J., M.M. Holland, M.C. Serreze, and J.J. Cassano, 2007: Response of Northern Hemisphere extratropical cyclone activity and associated precipitation to climate change, as represented by the Community Climate System Model. Journal of Geophysical Research - Biogeosciences, 112, G04S42.

Fiore, M.M.E., E.E. D'Onofrio, J.L. Pousa, E.J. Schnack, and G.R. Bertola, 2009: Storm surges and coastal impacts at Mar del Plata, Argentina. Continental Shelf Research, 29(14), 1643-1649.

Fischer, E.M. and C. Schär, 2009: Future changes in daily summer temperature variability: driving processes and role for temperature extremes. Climate Dynamics, 33(7-8), 917-935.

Fischer, E.M., and C. Schär, 2010: Consistent geographical patterns of changes in high-impact European heatwaves. Nature Geoscience, 3(6), 398-403.

Fischer, E.M., S.I. Seneviratne, D. Lüthi, and C. Schär, 2007a: The contribution of landatmosphere coupling to recent European summer heatwaves. Geophysical Research Letters, 34, L06707.

Fischer, E.M., S.I. Seneviratne, P.L. Vidale, D. Lüthi, and C. Schär, 2007b: Soil moistureatmosphere interactions during the 2003 European summer heatwave. Journal of Climate, 20(20), 5081-5099.

Fischer, L., H. Eisenbeiss, A. Kääb, C. Huggel, and W. Haeberli, 2011: Monitoring topographic changes in a periglacial high-mountain face using high-resolution DTMs, Monte Rosa East Face, Italian Alps. Permafrost and Periglacial Processes, 22(2), 140-152. 
Fleig, A.K., L.M. Tallaksen, H. Hisdal, and D.M. Hannah, 2011: Regional hydrological drought in north-western Europe: linking a new Regional Drought Area Index with weather types. Hydrological Processes, 25(7), 1163-1179.

Fogt, R.L., J. Perlwitz, A.J. Monaghan, D.H. Bromwich, J.M. Jones, and G.J. Marshall, 2009: Historical SAM variability. Part II: Twentieth-century variability and trends from reconstructions, observations, and the IPCC AR4 models. Journal of Climate, 22(20), 5346-5365.

Fontaine, B., P. Roucou, M. Gaetani, and R. Marteau, 2011: Recent changes in precipitation, ITCZ convection and northern tropical circulation over North Africa (1979-2007). International Journal of Climatology, 31(5), 633-648.

Forbes, D.L., G.S. Parkes, G.K. Manson, and L.A. Ketch, 2004: Storms and shoreline retreat in the southern Gulf of St. Lawrence. Marine Geology, 210(1-4), 169-204.

Founda, D., and C. Giannakopoulos, 2009: The exceptionally hot summer of 2007 in Athens, Greece - A typical summer in the future climate? Global and Planetary Change, 67(3-4), 227-236.

Fowler, H.J., and M. Ekstrom, 2009: Multi-model ensemble estimates of climate change impacts on UK seasonal precipitation extremes. International Journal of Climatology, 29(3), 385-416.

Fowler, H.J., and C.G. Kilsby, 2003: A regional frequency analysis of United Kingdom extreme rainfall from 1961 to 2000. Intl Journal of Climatology, 23(11), 1313-1334.

Fowler, H.J., and R.L. Wilby, 2010: Detecting changes in seasonal precipitation extremes using regional climate model projections: Implications for managing fluvial flood risk. Water Resources Research, 46, W0525.

Fowler, H.J., M. Ekstrom, S. Blenkinsop, and A.P. Smith, 2007a: Estimating change in extreme European precipitation using a multimodel ensemble. Journal of Geophysical Research - Atmospheres, 112, D18104.

Fowler, H.J., S. Blenkinsop, and C. Tebaldi, 2007b: Linking climate change modelling to impacts studies: recent advances in downscaling techniques for hydrological modelling. International Journal of Climatology, 27(12), 1547-1578.

Francou, B., E. Ramirez, B. Caceres, and J. Mendoza, 2000: Glacier evolution in the tropical Andes during the last decades of the 20th century: Chacaltaya, Bolivia, and Antizana, Ecuador. Ambio, 29(7), 416-422.

Frappier, A.B., T. Knutson, K.B. Liu, and K. Emanuel, 2007a: Perspective: Coordinating paleoclimate research on tropical cyclones with hurricane-climate theory and modelling. Tellus Series A - Dynamic Meteorology and Oceanography, 59(4), 529-537.

Frappier, A.B., D. Sahagian, S.J. Carpenter, L.A. Gonzalez, and B.R. Frappier, 2007b: Stalagmite stable isotope record of recent tropical cyclone events. Geology, 35(2), 111-114

Frederiksen, J.S., and C.S. Frederiksen, 2007: Interdecadal changes in southern hemisphere winter storm track modes. Tellus Series A - Dynamic Meteorology and Oceanography, 59(5), 599-617.

Frei, C. and C. Schär, 2001: Detection of probability of trends in rare events: Theory and application to heavy precipitation in the Alpine region. Journal of Climate, 14(7), 1568-1584.

Frei, C., J.H. Christensen, M. Deque, D. Jacob, R.G. Jones, and P.L. Vidale, 2003: Daily precipitation statistics in regional climate models: Evaluation and intercomparison for the European Alps. Journal of Geophysical Research -Atmospheres, 108, 4124.

Frei, C., R. Schöll, S. Fukutome, J. Schmidli, and P.L. Vidale, 2006: Future change of precipitation extremes in Europe: Intercomparison of scenarios from regional climate models. Journal of Geophysical Research - Atmospheres, 111, D06105.

Frey, H., W. Haeberli, A. Linsbauer, C. Huggel, and F. Paul, 2010: A multi-level strategy for anticipating future glacier lake formation and associated hazard potentials. Natural Hazards and Earth System Sciences, 10(2), 339-352.

Frich, P., L.V. Alexander, P.M. Della-Marta, B. Gleason, M. Haylock, A.M.G. Klein Tank, and T. Peterson, 2002: Observed coherent changes in climatic extremes during the second half of the twentieth century. Climate Research, 19(3), 193-212.

Friedlingstein, P., P. Cox, R. Betts, L. Bopp, W. von Bloh, V. Brovkin, P. Cadule, S. Doney, M. Eby, I. Fung, G. Bala, J. John, C. Jones, F. Joos, T. Kato, M. Kawamiya, W. Knorr, K. Lindsay, H.D. Matthews, T. Raddatz, P. Rayner, C. Reick, E. Roeckner, K.G. Schnitzler, R. Schnur, K. Strassmann, A.J. Weaver, C. Yoshikawa, and N. Zeng, 2006: Climate-carbon cycle feedback analysis: Results from the C4MIP model intercomparison. Journal of Climate, 19(14), 3337-3353.
Fu, C.B., S.Y. Wang, Z. Xiong, W.J. Gutowski, D.K. Lee, J.L. McGregor, Y. Sato, H. Kato, J.W. Kim, and M.S. Suh, 2005: Regional climate model intercomparison project for Asia. Bulletin of the American Meteorological Society, 86(2), 257-266.

Fujibe, F., N. Yamazaki, and K. Kobayashi, 2006: Long-term changes of heavy precipitation and dry weather in Japan (1901-2004). Journal of the Meteorological Society of Japan, 84(6), 1033-1046.

Fujihara, Y., K. Tanaka, T. Watanabe, T. Nagano, and T. Kojiri, 2008: Assessing the impacts of climate change on the water resources of the Seyhan River Basin in Turkey: Use of dynamically downscaled data for hydrologic simulations. Journal of Hydrology, 353(1-2), 33-48.

Funk, C., M.D. Dettinger, J.C. Michaelsen, J.P. Verdin, M.E. Brown, M. Barlow, and A. Hoell, 2008: Warming of the Indian Ocean threatens eastern and southern African food security but could be mitigated by agricultural development. Proceedings of the National Academy of Sciences, 105(32), 11081-11086.

Fyfe, J.C., 2003: Extratropical southern hemisphere cyclones: Harbingers of climate change? Journal of Climate, 16(17), 2802-2805.

Gallant, A.J.E., and D.J. Karoly, 2010: A combined climate extremes index for the Australian region. Journal of Climate, 23(23), 6153-6165.

Gallant, A.J.E., K.J. Hennessy, and J. Risbey, 2007: Trends in rainfall indices for six Australian regions: 1910-2005. Australian Meteorological Magazine, 56(4), 223-239.

Gan, M.A., V.B. Rao, and M.C.L. Moscati, 2006: South American monsoon indices. Atmospheric Science Letters, 6(4), 219-223.

García, J., M.C. Gallego, A. Serrano, and J. Vaquero, 2007: Trends in block-seasonal extreme rainfall over the Iberian Peninsula in the second half of the twentieth century. Journal of Climate, 20(1), 113-130.

Gastineau, G., and B.J. Soden, 2009: Model projected changes of extreme wind events in response to global warming. Geophysical Research Letters, 36, L10810.

Gavriliev, P.P., and P.V. Efremov, 2003: Effects of cryogenic processes on Yakutian landscapes under climate warming. In: Permafrost [Philips, M., S.M. Springman, and L.U. Arenson (eds.)]. Proceedings of the 8th International Conference on Permafrost, Zurich, Switzerland, 21-25 July 2003, Balkema, The Netherlands, pp. 277-282.

Gedney, N., P.M. Cox, R.A. Betts, O. Boucher, C. Huntingford, and P.A. Stott, 2006 Detection of a direct carbon dioxide effect in continental river runoff records. Nature, 439(7078), 835-838.

Geertsema, M., J.J. Clague, J.W. Schwab, and S.G. Evans, 2006: An overview of recent large catastrophic landslides in northern British Columbia, Canada. Engineering Geology, 83(1-3), 120-143.

Gehrels, W.R., D.F. Belknap, B.R. Pearce, and B. Gong, 1995: Modeling the contribution of $M(2)$ tidal amplification to the holocene rise of mean high water in the Gulf of Maine and the Bay of Fundy. Marine Geology, 124(1-4), 71-85.

Geng, Q. and M. Sugi, 2001: Variability of the North Atlantic cyclone activity in winter analyzed from NCEP-NCAR reanalysis data. Journal of Climate, 14(18), 3863-3873.

Gerten, D., S. Rost, W. von Bloh, and W. Lucht, 2008: Causes of change in 20th century global river discharge. Geophysical Research Letters, 35, L20405.

Giannakopoulos, C., P. Le Sager, M. Bindi, M. Moriondo, E. Kostopoulou, and C.M. Goodess, 2009: Climatic changes and associated impacts in the Mediterranean resulting from a $2^{\circ} \mathrm{C}$ global warming. Global and Planetary Change, 68(3) 209-224.

Giannini, A., R. Saravanan, and P. Chang, 2003: Oceanic forcing of Sahel rainfall on interannual to interdecadal time scales. Science, 302(5647), 1027-1030.

Giannini, A., M. Biasutti, and M.M. Verstraete, 2008: A climate model-based review of drought in the Sahel: Desertification, the re-greening and climate change. Global and Planetary Change, 64(3-4), 119-128.

Gibelin, A.L., and M. Deque, 2003: Anthropogenic climate change over the Mediterranean region simulated by a global variable resolution model. Climate Dynamics, 20(4), 327-339.

Gillett, N.P. and P.A. Stott, 2009: Attribution of anthropogenic influence on seasonal sea level pressure. Geophysical Research Letters, 36, L23709.

Gillett, N.P., R.J. Allan, and T.J. Ansell, 2005: Detection of external influence on sea level pressure with a multi-model ensemble. Geophysical Research Letters, 32 , L19714. 
Gillett, N.P., P.A. Stott, and B.D. Santer, 2008a: Attribution of cyclogenesis region sea surface temperature change to anthropogenic influence. Geophysical Research Letters, 35, L09707.

Gillett, N.P., D.A. Stone, P.A. Stott, T. Nozawa, A.Y. Karpechko, G.C. Hegerl, M.F. Wehner, and P.D. Jones, 2008b: Attribution of polar warming to human influence. Nature Geoscience, 1, 750-754.

Ginoux, P., J.M. Prospero, O. Torres, and M. Chin, 2004: Long-term simulation of global dust distribution with the GOCART model: correlation with North Atlantic Oscillation. Environmental Modelling \& Software, 19(2), 113-128.

Giorgi, F., 2006: Climate change hot-spots. Geophysical Research Letters, 33, L08707.

Giorgi, F. and P. Lionello, 2008: Climate change projections for the Mediterranean region. Global and Planetary Change, 63(2-3), 90-104.

Giorgi, F., C. Jones, and G.R. Asra, 2009: Addressing climate information needs at the regional level: the CORDEX framework. WMO Bulletin, 58(3), 175-183.

Glade, T., 1998: Establishing the frequency and magnitude of landslide-triggering rainstorm events in New Zealand. Environmental Geology, 55(2), 160-174.

Gleason, K.L., J.H. Lawrimore, D.H. Levinson, and T.R. Karl, 2008: A revised U.S. climate extremes index. Journal of Climate, 21(10), 2124-2137.

Gong, S.L., X.Y. Zhang, T.L. Zhao, X.B. Zhang, L.A. Barrie, I.G. McKendry, and C.S. Zhao, 2006: A simulated climatology of Asian dust aerosol and its trans-Pacific transport. Part II: Interannual variability and climate connections. Journal of Climate, 19(1), 104-122.

Goodkin, N.F., K.A. Hughen, S.C. Doney, and W.B. Curry, 2008: Increased multidecadal variability of the North Atlantic Oscillation since 1781. Nature Geoscience, 1, 844-848.

Goubanova, K. and L. Li, 2007: Extremes in temperature and precipitation around the Mediterranean basin in an ensemble of future climate scenario simulations. Global and Planetary Change, 57(1-2), 27-42.

Goudie, A.S., 2009: Dust storms: Recent developments. Journal of Environmental Management, 90(1), 89-94.

Goudie, A.S., and N.J. Middleton, 1992: The changing frequency of dust storms through time. Climatic Change, 20(3), 197-225.

Grabemann, I., and R. Weisse, 2008: Climate change impact on extreme wave conditions in the North Sea: an ensemble study. Ocean Dynamics, 58(3-4), 199-212.

Graham, L.P., J. Andreasson, and B. Carlsson, 2007: Assessing climate change impacts on hydrology from an ensemble of regional climate models, model scales and linking methods - a case study on the Lule River basin. Climatic Change, 81(S1), 293-307.

Graham, N.E. and H.F. Diaz, 2001: Evidence for intensification of North Pacific winter cyclones since 1948. Bulletin of the American Meteorological Society, 82(9), 1869-1893.

Gravelle, G. and N. Mimura, 2008: Vulnerability assessment of sea-level rise in Viti Levu, Fiji Islands. Sustainability Science, 3(2), 171-180.

Green, D., L. Alexander, K.L. Mclnnes, J. Church, N. Nicholls, and W. White, 2009: An assessment of climate change impacts and adaptation for the Torres Strait Islands, Australia. Climatic Change, 102(3-4), 405-433.

Greene, A.M., A. Giannini, and S.E. Zebiak, 2009: Drought return times in the Sahel: A question of attribution. Geophysical Research Letters, 36, L12701.

Greeves, C.Z., V.D. Pope, R.A. Stratton, and G.M. Martin, 2007: Representation of Northern Hemisphere winter storm tracks in climate models. Climate Dynamics, 28(7-8), 683-702.

Grell, G.A., L. Schade, R. Knoche, A. Pfeiffer, and J. Egger, 2000: Nonhydrostatic climate simulations of precipitation over complex terrain. Journal of Geophysical Research - Atmospheres, 105(D24), 29595-29608.

Griffiths, G.M., M.J. Salinger, and I. Leleu, 2003: Trends in extreme daily rainfall across the South Pacific and relationship to the South Pacific Convergence Zone. International Journal of Climatology, 23(8), 847-869.

Griffiths, G.M., L.E. Chambers, M.R. Haylock, M.J. Manton, N. Nicholls, H.J. Baek, Y. Choi, P.M. Della-Marta, A. Gosai, N. Iga, R. Lata, V. Laurent, L. Maitrepierre, H. Nakamigawa, N. Ouprasitwong, D. Solofa, L. Tahani, D.T. Thuy, L. Tibig, B. Trewin, K. Vediapan, and P. Zhai, 2005: Change in mean temperature as a predictor of extreme temperature change in the Asia-Pacific region. International Journal of Climatology, 25(10), 1301-1330.
Grimm, A.M. and R.G. Tedeschi, 2009: ENSO and extreme rainfall events in South America. Journal of Climate, 22(7), 1589-1609.

Grimm, A.M., J. Pal, and F. Giorgi, 2007: Connection between spring conditions and peak summer monsoon rainfall in South America: Role of soil moisture, surface temperature, and topography in eastern Brazil. Journal of Climate, 20(24), 5929-5945

Grinsted, A., A.J. Moore, and S. Jevrejeva, 2010: Reconstructing sea level from paleo and projected temperatures 200 to 2100 AD. Climate Dynamics, 34(4), 461-472.

Groisman, P.Y., and R.W. Knight, 2008: Prolonged dry episodes over the conterminous United States: New tendencies emerging during the last 40 years. Journal of Climate, 21(9), 1850-1862.

Gruber, S., and W. Haeberli, 2007: Permafrost in steep bedrock slopes and its temperature-related destabilization following climate change. Journal of Geophysical Research - Earth Surface, 112, F02S18.

Gruber, S., M. Hoelzle, and W. Haeberli, 2004: Permafrost thaw and destabilization of Alpine rock walls in the hot summer of 2003. Geophysical Research Letters, 31, L13504.

Gualdi, S., E. Scoccimarro, and A. Navarra, 2008: Changes in tropical cyclone activity due to global warming: Results from a high-resolution coupled general circulation model. Journal of Climate, 21(20), 5204-5228.

Guilyardi, E., A. Wittenberg, A. Fedorov, M. Collins, C.Z. Wang, A. Capotondi, G.J. van Oldenborgh, and T. Stockdale, 2009: Understanding El Niño in oceanatmosphere General Circulation Models: Progress and challenges. Bulletin of the American Meteorological Society, 90(3), 325-340.

Gulev, S.K. and V. Grigorieva, 2004: Last century changes in ocean wind wave height from global visual wave data. Geophysical Research Letters, 31, L24302.

Gulev, S.K., O. Zolina, and S. Grigoriev, 2001: Extratropical cyclone variability in the Northern Hemisphere winter from the NCEP/NCAR reanalysis data. Climate Dynamics, 17(10), 795-809.

Guo, H., M. Xu, and Q. Hu, 2011: Changes in near-surface wind speed in China: 1969-2005. International Journal of Climatology, 31(2), 349-358.

Gutowski, W.J., L.E. Branscome, and D.A. Stewart, 1992: Life-cycles of moist baroclinic eddies. Journal of the Atmospheric Sciences, 49(4), 306-319.

Gutowski, W.J., S.G. Decker, R.A. Donavon, Z.T. Pan, R.W. Arritt, and E.S. Takle, 2003: Temporal-spatial scales of observed and simulated precipitation in central U.S. climate. Journal of Climate, 16(22), 3841-3847.

Gutowski, W.J., G.C. Hegerl, G.J. Holland, T.R. Knutson, L.O. Mearns, R.J. Stouffer, P.J. Webster, M.F. Wehner, and F.W. Zwiers, 2008a: Causes of observed changes in extremes and projections of future changes. In: Weather and Climate Extremes in a Changing Climate. Regions of Focus: North America, Hawaii, Caribbean, and U.S. Pacific Islands. [Karl, T.R., G.A. Meehl, D.M. Christopher, S.J. Hassol, A.M. Waple, and W.L. Murray (eds.)]. A Report by the U.S. Climate Change Science Program and the Subcommittee on Global Change Research, Washington, DC, pp. 81-116.

Gutowski, W.J., S.S. Willis, J.C. Patton, B.R.J. Schwedler, R.W. Arritt, and E.S. Takle, 2008b: Changes in extreme, cold-season synoptic precipitation events under global warming. Geophysical Research Letters, 35, L20710.

Haarsma, R.J., F. Selten, B.V. Hurk, W. Hazeleger, and X.L. Wang, 2009: Drier Mediterranean soils due to greenhouse warming bring easterly winds over summertime central Europe. Geophysical Research Letters, 36, L04705.

Haeberli, W., 1983: Frequency and characteristics of glacier floods in the Swiss Alps. Annals of Glaciology, 4, 85-90.

Haeberli, W., and C.R. Burn, 2002: Natural hazards in forests: glacier and permafrost effects as related to climate change. In: Environmental Change and Geomorphic Hazards in Forests, IUFRO Research Series 9 [Sidle, R.C. (ed.)]. CABI Publishing, Wallingford, UK, pp. 167-202.

Haeberli, W. and M. Funk, 1991: Borehole temperatures at the Colle Gnifetti coredrilling site (Monte Rosa, Swiss Alps). Journal of Glaciology, 37(125), 37-46.

Haeberli, W. and R. Hohmann, 2008: Climate, glaciers and permafrost in the Swiss Alps 2050: scenarios, consequences and recommendations. In: Permafrost on a Warming Planet: Impacts on Ecosystems, Infrastructure and Climate [Kane, D.L., and K.M. Hinkel (eds.)]. Proc. of the 9th Intl. Conf. on Permafrost at University of Alaska, Fairbanks, 9 June - 3 July 2008, Inst. of Northern Engineering, pp. 607-612. 
Haeberli, W., M. Wegmann, and D. Vonder Mühll, 1997: Slope stability problems related to glacier shrinkage and permafrost degradation in the Alps. Eclogae Geologicae Helvetiae, 90(3), 407-414.

Haeberli, W., A. Kääb, D. Vonder Mühll, and P. Teysseire, 2001: Prevention of outburst floods from periglacial lakes at Grubengletscher, Valais, Swiss Alps. Journal of Glaciology, 47(156), 111-122.

Haeberli, W., C. Huggel, A. Kääb, S. Zgraggen-Oswald, A. Polkvoj, I. Galushkin, I. Zotikov, and N. Osokin, 2004: The Kolka-Karmadon rock/ice slide of 20 September 2002: an extraordinary event of historical dimensions in North Ossetia, Russian Caucasus. Journal of Glaciology, 50(171), 533-546.

Haerter, J.0. and P. Berg, 2009: Unexpected rise in extreme precipitation caused by a shift in rain type? Nature Geoscience, 2(6), 372-373.

Haigh, I., R. Nicholls, and N. Wells, 2009: Mean sea level trends around the English Channel over the 20th century and their wider context. Continental Shelf Research, 29(17), 2083-2098.

Haigh, I., R. Nicholls, and N. Wells, 2010: Assessing changes in extreme sea levels: Application to the English Channel, 1900-2006. Continental Shelf Research, 30(9), 1042-1055

Hall, A., X. Qu, and J.D. Neelin, 2008: Improving predictions of summer climate change in the United States. Geophysical Research Letters, 35, L01702.

Hampel, A., R. Hetzel, and G. Maniatis, 2010: Response of faults to climate-driven changes in ice and water volumes on Earth's surface. Philosophical Transactions of the Royal Society A: Mathematical, Physical and Engineering Sciences, 368(1919), 2501-2517.

Hanna, E., J. Cappelen, R. Allan, T. Jonsson, F. Le Blancq, T. Lillington, and K. Hickey, 2008: New insights into North European and North Atlantic surface pressure variability, storminess, and related climatic change since 1830. Journal of Climate, 21(24), 6739-6766.

Hannaford, J., and T.J. Marsh, 2008: High-flow and flood trends in a network of undisturbed catchments in the UK. International Journal of Climatology, 28(10), 1325-1338.

Hanson, C.E., J.P. Palutikof, and T.D. Davies, 2004: Objective cyclone climatologies of the North Atlantic - a comparison between the ECMWF and NCEP Reanalyses. Climate Dynamics, 22(6-7), 757-769.

Harper, B., T. Hardy, L. Mason, and R. Fryar, 2009: Developments in storm tide modelling and risk assessment in the Australian region. Natural Hazards, 51(1), 225-238.

Harris, C., L.U. Arenson, H.H. Christiansen, B. Etzelmüller, R. Frauenfelder, S. Gruber, W. Haeberli, C. Hauck, M. Hölzle, O. Humlum, K. Isaksen, A. Kääb, M.A. KernLütschg, M. Lehning, N. Matsuoka, J.B. Murton, J. Nötzli, M. Phillips, N. Ross, M. Seppälä, S.M. Springman, and D. Vonder Mühll, 2009: Permafrost and climate in Europe: Monitoring and modelling thermal, geomorphological and geotechnical responses. Earth-Science Reviews, 92(3-4), 117-171.

Haug, G.H., K.A. Hughen, D.M. Sigman, L.C. Peterson, and U. Röhl, 2001: Southward migration of the Intertropical Convergence Zone through the Holocene. Science, 293(5533), 1304-1308.

Haugen, J.E. and T. Iversen, 2008: Response in extremes of daily precipitation and wind from a downscaled multi-model ensemble of anthropogenic global climate change scenarios. Tellus Series A - Dynamic Meteorology and Oceanography, 60(3), 411-426.

Hawkins, E. and R. Sutton, 2009: The potential to narrow uncertainty in regional climate predictions. Bulletin of the American Meteorological Society, 90(8), 1095-1107.

Hawkins, E. and R. Sutton, 2011: The potential to narrow uncertainty in projections of regional precipitation change. Climate Dynamics, 37(1-2), 407-418.

Hay, L.E., M.P. Clark, M. Pagowski, G.H. Leavesley, and W.J. Gutowski, 2006: One-way coupling of an atmospheric and a hydrologic model in Colorado. Journal of Hydrometeorology, 7(4), 569-589.

Haylock, M.R. and C.M. Goodess, 2004: Interannual variability of European extreme winter rainfall and links with mean large-scale circulation. International Journal of Climatology, 24(6), 759-776.

Haylock, M.R., G.C. Cawley, C. Harpham, R.L. Wilby, and C.M. Goodess, 2006a: Downscaling heavy precipitation over the United Kingdom: A comparison of dynamical and statistical methods and their future scenarios. International Journal of Climatology, 26(10), 1397-1415.
Haylock, M.R., T.C. Peterson, L.M. Alves, T. Ambrizzi, Y.M.T. Anunciacao, J. Baez, V.R. Barros, M.A. Berlato, M. Bidegain, G. Coronel, V. Corradi, V.J. Garcia, A.M. Grimm, D. Karoly, J.A. Marengo, M.B. Marino, D.F. Moncunill, D. Nechet, J. Quintana, E. Rebello, M. Rusticucci, J.L. Santos, I. Trebejo, and L.A. Vincent, 2006b: Trends in total and extreme South American rainfall in 1960-2000 and links with sea surface temperature. Journal of Climate, 19(8), 1490-1512.

Haylock, M.R., N. Hofstra, A. Tank, E.J. Klok, P.D. Jones, and M. New, 2008: A European daily high-resolution gridded data set of surface temperature and precipitation for 1950-2006. Journal of Geophysical Research - Atmospheres, 113(D20119).

Hegerl, G.C., F.W. Zwiers, P.A. Stott, and V.V. Kharin, 2004: Detectability of anthropogenic changes in annual temperature and precipitation extremes. Journal of Climate, 17(19), 3683-3700.

Hegerl, G.C., F.W. Zwiers, P. Braconnot, N.P. Gillett, Y. Luo, J.A. Marengo Orsini, N. Nicholls, J.E. Penner, and P.A. Stott, 2007: Understanding and Attributing Climate Change. In: Climate Change 2007: The Physical Science Basis. Contribution of Working Group I to the Fourth Assessment Report of the Intergovernmental Panel on Climate Change [Solomon, S., D. Qin, M. Manning, Z. Chen, M. Marquis, K.B. Averyt, M. Tignor and H.L. Miller (eds.)]. Cambridge University Press, Cambridge, UK, pp. 663-745.

Hegerl, G.C., O. Hoegh-Guldberg, G. Casassa, M.P. Hoerling, R.S. Kovats, C. Parmesan, D.W. Pierce, and P.A. Stott, 2010: Good practice guidance paper on detection and attribution related to anthropogenic climate change. In: Meeting Report of the Intergovernmental Panel on Climate Change Expert Meeting on Detection and Attribution of Anthropogenic Climate Change [Stocker, T.F., C.B. Field, D. Qin, V. Barros, G.-K. Plattner, M. Tignor, P.M. Midgley, and K.L. Ebi (eds.)]. IPCC Working Group I Technical Support Unit, University of Bern, Bern, Switzerland.

Hegglin, E. and C. Huggel, 2008: An integrated assessment of vulnerability to glacial hazards. Mountain Research and Development, 28(3), 299-309.

Heim Jr., R.R., 2002: A review of twentieth-century drought indices used in the United States. Bulletin of the American Meteorological Society, 83(8), 1149-1165.

Held, I.M. and B.J. Soden, 2006: Robust responses of the hydrological cycle to global warming. Journal of Climate, 19(21), 5686-5699.

Hemer, M.A., 2010: Historical trends in Southern Ocean storminess: Long-term variability of extreme wave heights at Cape Sorell, Tasmania. Geophysical Research Letters, 37, L18601.

Hemer, M.A., J.A. Church, and J.R. Hunter, 2010: Variability and trends in the directional wave climate of the Southern Hemisphere. International Journal of Climatology, 30(4), 475-491.

Hendon, H.H., D.W.J. Thompson, and M.C. Wheeler, 2007: Australian rainfall and surface temperature variations associated with the Southern Hemisphere annular mode. Journal of Climate, 20(11), 2452-2467.

Herath, S. and U. Ratnayake, 2004: Monitoring rainfall trends to predict adverse impacts - a case study from Sri Lanka (1964-1993). Global Environmental Change - Human and Policy Dimensions, 14(1), 71-79.

Hewitson, B.C. and R.G. Crane, 2006: Consensus between GCM climate change projections with empirical downscaling: Precipitation downscaling over South Africa. International Journal of Climatology, 26(10), 1315-1337.

Hidalgo, H.G., T. Das, M.D. Dettinger, D.R. Cayan, D.W. Pierce, T.P. Barnett, G. Bala, A. Mirin, A.W. Wood, C. Bonfils, B.D. Santer, and T. Nozawa, 2009: Detection and attribution of streamflow timing changes to climate change in the western United States. Journal of Climate, 22(13), 3838-3855.

Hinkel, J. and R.J.T. Klein, 2009: Integrating knowledge to assess coastal vulnerability to sea-level rise: The development of the DIVA tool. Global Environmental Change - Human and Policy Dimensions, 19(3), 384-395.

Hinzman, L.D., N.D. Bettez, W.R. Bolton, F.S. Chapin, M.B. Dyurgerov, B.G. Fastie, B. Griffith, R.D. Hollister, A. Hope, H.P. Huntington, A.M. Jenson, G.J. Jia, R. Jorgenson, D. Kane, D.R. Klein, G. Kofinas, A.H. Lynch, A.H. Lloyd, D. McGuire, F.E. Nelson, W.C. Oechel, T.E. Osterkamp, C.H. Racine, V.E. Romanovsky, R.S. Stone, D.A. Stow, M. Sturm, C.E. Tweedies, G.L. Vourlitis, M.D. Walker, D.A. Walker, P.J. Webber, J.M. Welker, K.S. Winker, and K. Yoshikawa, 2005: Evidence and implications of recent climate change in northern Alaska and other arctic regions. Climatic Change, 72(3), 251-298. 
Hirabayashi, Y., S. Kanae, K. Motoya, K. Masuda, and P. Doll, 2008a: A 59-year (1948-2006) global meteorological forcing data set for land surface models. Part II: Global snowfall estimation. Hydrological Research Letters, 2, 65-69.

Hirabayashi, Y., S. Kanae, S. Emori, T. Oki, and M. Kimoto, 2008b: Global projections of changing risks of floods and droughts in a changing climate. Hydrological Sciences Journal, 53(4), 754-772.

Hirsch, M.E., A.T. DeGaetano, and S.J. Colucci, 2001: An East Coast winter storm climatology. Journal of Climate, 14(5), 882-899.

Hirschi, M., S.I. Seneviratne, V. Alexandrov, F. Boberg, C. Boroneant, O.B. Christensen, H. Formayer, B. Orlowsky, and P. Stepanek, 2011: Observational evidence for soil-moisture impact on hot extremes in southeastern Europe. Nature Geoscience, 4(1), 17-21.

Hodges, K.I., B.J. Hoskins, J. Boyle, and C. Thorncroft, 2003: A comparison of recent reanalysis datasets using objective feature tracking: Storm tracks and tropical easterly waves. Monthly Weather Review, 131(9), 2012-2037.

Hoegh-Guldberg, 0., 1999: Climate change, coral bleaching and the future of the world's coral reefs. Marine and Freshwater Research, 50(8), 839-866.

Hoelzle, M., G. Darms, and S. Suter, 2010: Evidence of accelerated englacial warming in the Monte Rosa area, Switzerland/Italy. The Cryosphere Discussions, 4(4), 2277-2305.

Hoerling, M., J. Hurrell, J. Eischeid, and A. Phillips, 2006: Detection and attribution of twentieth-century northern and southern African rainfall change. Journal of Climate, 19(16), 3989-4008.

Hohenegger, C., P. Brockhaus, and C. Schär, 2008: Towards climate simulations at cloud-resolving scales. Meteorology Zeitschrift, 17(4), 383-394.

Hohenegger, C., P. Brockhaus, C.S. Bretherton, and C. Schar, 2009: The soil moistureprecipitation feedback in simulations with explicit and parameterized convection. Journal of Climate, 22(19), 5003-5020.

Holland, G.J. and P.J. Webster, 2007: Heightened tropical cyclone activity in the North Atlantic: Natural variability or climate trend? Philosophical Transactions of the Royal Society A, 365, 2695-2716.

Hope, P.K., W. Drosdowsky, and N. Nicholls, 2006: Shifts in the synoptic systems influencing southwest Western Australia. Climate Dynamics, 26(7-8), 751-764.

Horton, R., C. Herweijer, C. Rosenzweig, J. Liu, V. Gornitz, and A.C. Ruane, 2008: Sea level rise projections for current generation CGCMs based on the semi-empirical method. Geophysical Research Letters, 35, L02715.

Hossain, F., I. Jeyachandran, and R. Pielke Jr, 2009: Have large dams altered extreme precipitation patterns. EOS Transactions (AGU), 90(48), 453.

Hourdin, F., I. Musat, F. Guichard, P.M. Ruti, F. Favot, M.A. Filiberti, M. Pham, J.Y. Grandpeix, J. Polcher, P. Marquet, A. Boone, J.P. Lafore, J.L. Redelsperger, A. Dell'aquila, T.L. Doval, A.K. Traore, and H. Gallee, 2010: AMMA-model intercomparison project. Bulletin of the American Meteorological Society, 91(1), 95-104.

Huebener, H., U. Cubasch, U. Langematz, T. Spangehl, F. Niehoerster, I. Fast, and M. Kunze, 2007: Ensemble climate simulations using a fully coupled oceantroposphere-stratosphere general circulation model. Philosophical Transactions of the Royal Society A, 365(1857), 2089-2101.

Huggel, C., 2009: Recent extreme slope failures in glacial environments: effects of thermal perturbation. Quaternary Science Reviews, 28(11-12), 1119-1130.

Huggel, C., W. Haeberli, A. Kääb, D. Bieri, and S. Richardson, 2004: An assessment procedure for glacial hazards in the Swiss Alps. Canadian Geotechnical Journal, 41(6), 1068-1083.

Huggel, C., S. Zgraggen-Oswald, W. Haeberli, A. Kääb, A. Polkvoj, I. Galushkin, and S.G. Evans, 2005: The 2002 rock/ice avalanche at Kolka/Karmadon, Russian Caucasus: assessment of extraordinary avalanche formation and mobility, and application of QuickBird satellite imagery. Natural Hazards and Earth System Sciences, 5(2), 173-187.

Huggel, C., J. Caplan-Auerbach, C.F. Waythomas, and R.L. Wessels, 2007: Monitoring and modeling ice-rock avalanches from ice-capped volcanoes: A case study of frequent large avalanches on Iliamna Volcano, Alaska. Journal of Volcanology and Geothermal Research, 168(1-4), 114-136.

Huggel, C., J. Caplan-Auerbach, and R. Wessels, 2008: Recent extreme avalanches: Triggered by climate change. Eos Transactions (AGU), 89(47), 469-470.
Huggel, C., N. Salzmann, S. Allen, J. Caplan-Auerbach, L. Fischer, W. Haeberli, C. Larsen, D. Schneider, and R. Wessels, 2010: Recent and future warm extreme events and high-mountain slope failures. Philosophical Transactions of the Royal Society A, 368, 2435-2459.

Hulme, M., R. Doherty, T. Ngara, M. New, and D. Lister, 2001: African climate change: 1900-2100. Climate Research, 17(2), 145-168.

Hundecha, Y. and A. Bardossy, 2008: Statistical downscaling of extreme of daily precipitation and temperature and construction of their future scenarios. International Journal of Climatology, 28(5), 589-610.

Hundecha, Y., A. St-Hilaire, T.B.M.J. Ouarda, S. El Adlouni, and P. Gachon, 2008: A nonstationary extreme value analysis for the assessment of changes in extreme annual wind speed over the Gulf of St. Lawrence, Canada. Journal of Applied Meteorology and Climatology, 47(11), 2745-2759.

Hungate, B.A., J.S. Dukes, M.R.L.Y. Shaw, and C.B. Field, 2003: Nitrogen and climate change. Science, 302(5650), 1512-1513.

Hunter, J., 2010: Estimating sea-level extremes under conditions of uncertain sealevel rise. Climatic Change, 99(3-4), 331-350.

Huss, M., D. Farinotti, A. Bauder, and M. Funk, 2008: Modelling runoff from highly glacierized alpine drainage basins in a changing climate. Hydrological Processes, 22(19), 3888-3902.

Im, E.S. and W.-T. Kwon, 2007: Characteristics of extreme climate sequences over Korea using a regional climate change scenario. SOLA, 3, 17-20.

Im, E.S., J.B. Ahn, W.T. Kwon, and F. Giorgi, 2008: Multi-decadal scenario simulation over Korea using a one-way double-nested regional climate model system. Part 2: Future climate projection (2021-2050). Climate Dynamics, 30(2-3), 239-254.

Im, E.S., I.W. Jung, and D.H. Bae, 2011: The temporal and spatial structures of recent and future trends in extreme indices over Korea from a regional climate projection. International Journal of Climatology, 31(1), 72-86.

Inoue, J., J.P. Liu, J.O. Pinto, and J.A. Curry, 2006: Intercomparison of Arctic Regional Climate Models: Modeling clouds and radiation for SHEBA in May 1998. Journal of Climate, 19(17), 4167-4178.

IPCC, 2007a: Climate Change 2007: The Physical Science Basis. Contribution of Working Group I to the Fourth Assessment Report of the Intergovernmental Panel on Climate Change [Solomon, S., D. Qin, M. Manning, Z. Chen, M. Marquis, K.B. Averyt, M. Tignor, and H.L. Miller (eds.)]. Cambridge University Press, Cambridge, UK, $996 \mathrm{pp}$

IPCC, 2007b: Summary for Policymakers. In: Climate Change 2007: The Physical Science Basis. Contribution of Working Group I to the Fourth Assessment Report of the Intergovernmental Panel on Climate Change [Solomon, S., D. Qin, M. Manning, Z. Chen, M. Marquis, K.B. Averyt, M. Tignor and H.L. Miller (eds.)]. Cambridge University Press, Cambridge, UK, pp. 1-18.

Irish, J.L. and D.T. Resio, 2010: A hydrodynamics-based surge scale for hurricanes. Ocean Engineering, 37(1), 69-81.

Iverson, R.M., 2000: Landslide triggering by rain infiltration. Water Resources Research, 36(7), 1897-1910.

Jakob, D., 2010: Challenges in developing a high-quality surface wind-speed data set for Australia. Australian Meteorological and Oceanographic Journal, 60(4), 227-236.

Jakob, M. and H. Weatherly, 2003: A hydroclimatic threshold for landslide initiation on the North Shore Mountains of Vancouver, British Columbia. Geomorphology, 54(3-4), 137-156

Janicot, S., 2009: A comparison of Indian and African monsoon variability at different time scales. Comptes Rendus Geoscience, 341(8-9), 575-590.

Jansen, E., J. Overpeck, K.R. Briffa, J.C. Duplessy, F. Joos, V. Masson-Delmotte, V. Olago, B. Otto-Bliesner, W.R. Peltier, S. Rahmstorf, R. Ramesh, D. Raynaud, D. Rind, O. Solomina, R. Villalba, and D. Zhang, 2007: Palaeoclimate. In: Climate Change 2007: The Physical Science Basis. Contribution of Working Group I to the Fourth Assessment Report of the Intergovernmental Panel on Climate Change [Solomon, S., D. Qin, M. Manning, Z. Chen, M. Marquis, K.B. Averyt, M. Tignor and H.L. Miller (eds.)]. Cambridge University Press, Cambridge, UK, pp. 433-497.

Jiang, T., Z.W. Kundzewicz, and B. Su, 2008: Changes in monthly precipitation and flood hazard in the Yangtze River Basin, China. International Journal of Climatology, 28(11), 1471-1481. 
Jiang, Y., Y. Luo, Z.C. Zhao, and S.W. Tao, 2010a: Changes in wind speed over China during 1956-2004. Theoretical and Applied Climatology, 99(3-4), 421-430.

Jiang, Y., Y. Luo, Z.C. Zhao, Y. Shi, Y.L. Xu, and J.H. Zhu, 2010b: Projections of wind changes for 21st century in China by three regional climate models. Chinese Geographical Science, 20(3), 226-235.

Johnson, N.C. and S.-P. Xie, 2010: Changes in the sea surface temperature threshold for tropical convection. Nature Geoscience, 3(12), 842-845.

Joly, M. and A. Voldoire, 2010: Role of the Gulf of Guinea in the inter-annual variability of the West African monsoon: what do we learn from CMIP3 coupled simulations? International Journal of Climatology, 30(12), 1843-1856.

Jomelli, V., V.P. Pech, C. Chochillon, and D. Brunstein, 2004: Geomorphic variations of debris flows and recent climatic change in the French Alps. Climatic Change, 64(1), 77-102.

Jones, B.M., C.D. Arp, M.T. Jorgenson, K.M. Hinkel, J.A. Schmutz, and P.L. Flint, 2009: Increase in the rate and uniformity of coastline erosion in Arctic Alaska. Geophysical Research Letters, 36, L03503.

Jones, G.S., P.A. Stott, and N. Christidis, 2008: Human contribution to rapidly increasing frequency of very warm Northern Hemisphere summers. Journal of Geophysical Research, 113, D02109.

Jorgenson, M.T., Y.L. Shur, and E.R. Pullman, 2006: Abrupt increase in permafrost degradation in Arctic Alaska. Geophysical Research Letters, 33, L02503.

Joseph, R. and S. Nigam, 2006: ENSO evolution and teleconnections in IPCC's twentieth-century climate simulations: realistic representation? Journal of Climate, 19(17), 4360-4377.

Jull, M. and D. McKenzie, 1996: The effect of deglaciation on mantle melting beneath Iceland. Journal of Geophysical Research - Solid Earth, 101(B10), 21815-21828.

Jung, M., M. Reichstein, P. Ciais, S.I. Seneviratne, J. Sheffield, M.L. Goulden, G. Bonan, A. Cescatti, J.Q. Chen, R. de Jeu, A.J. Dolman, W. Eugster, D. Gerten, D. Gianelle, N. Gobron, J. Heinke, J. Kimball, B.E. Law, L. Montagnani, Q.Z. Mu, B. Mueller, K. Oleson, D. Papale, A.D. Richardson, O. Roupsard, S. Running, E. Tomelleri, N. Viovy, U. Weber, C. Williams, E. Wood, S. Zaehle, and K. Zhang, 2010: Recent decline in the global land evapotranspiration trend due to limited moisture supply. Nature, 467(7318), 951-954.

Jung, T., S.K. Gulev, I. Rudeva, and V. Soloviov, 2006: Sensitivity of extratropical cyclone characteristics to horizontal resolution in the ECMWF model. Quarterly Journal of the Royal Meteorological Society, 132(619), 1839-1857.

Kääb, A., R. Frauenfelder, and I. Roer, 2007: On the response of rockglacier creep to surface temperature increase. Global and Planetary Change, 56(1-2), 172-187.

Kallis, G., 2008: Droughts. Annual Review of Environment and Resources, 33, 85-118.

Kamiguchi, K., A. Kitoh, T. Uchiyama, R. Mizuta, and A. Noda, 2006: Changes in precipitation-based extremes indices due to global warming projected by a global 20-km-mesh atmospheric model. SOLA, 2, 64-67.

Kanada, S., M. Nakano, and T. Kato, 2010a: Changes in mean atmospheric structures around Japan during July due to global warming in regional climate experiments using a cloud system resolving model. Hydrological Research Letters, 4, 11-14.

Kanada, S., M. Nakano, and T. Kato, 2010b: Climatological characteristics of daily precipitation over Japan in the Kakushin regional climate experiments using a non-hydrostatic 5-km-mesh model: Comparison with an outer global 20-kmmesh atmospheric climate model. SOLA, 6, 117-120.

Kang, I.S. and J. Shukla, 2006: Dynamic seasonal prediction and predictability of the monsoon. In: The Asian Monsoon [Wang, B. (ed.)]. Springer/Praxis, New York, NY, pp. 585-612.

Karl, T.R., G.A. Meehl, D.M. Christopher, S.J. Hassol, A.M. Waple, and W.L. Murray, 2008: Weather and Climate Extremes in a Changing Climate. Regions of Focus: North America, Hawaii, Caribbean, and U.S. Pacific Islands. A Report by the U.S. Climate Change Science Program and the Subcommittee on Global Change Research, Washington, DC, $164 \mathrm{pp}$.

Karl, T.R., J.M. Melillo, and T.C. Peterson (eds.), 2009: Global Climate Change Impacts in the United States. Cambridge University Press, New York, NY, 189 pp.

Karoly, D.J. and Q.G. Wu, 2005: Detection of regional surface temperature trends. Journal of Climate, 18(21), 4337-4343.
Kaser, G., J.G. Cogley, M.B. Dyurgerov, M.F. Meier, and A. Ohmura, 2006: Mass balance of glaciers and ice caps: Consensus estimates for 1961-2004. Geophysical Research Letters, 33, L19501.

Kay, A.L., H.N. Davies, V.A. Bell, and R.G. Jones, 2009: Comparison of uncertainty sources for climate change impacts: flood frequency in England. Climatic Change, 92(1-2), 41-63.

Kay, G. and R. Washington, 2008: Future southern African summer rainfall variability related to a southwest Indian Ocean dipole in HadCM3. Geophysical Research Letters, 35, L12701.

Keeling, R.F., A. Kortzinger, and N. Gruber, 2010: Ocean deoxygenation in a warming world. Annual Review of Marine Science, 2, 199-229.

Keenlyside, N.S., M. Latif, J. Jungclaus, L. Kornblueh, and E. Roeckner, 2008: Advancing decadal-scale climate prediction in the North Atlantic sector. Nature, 453, 84-88.

Kellogg, C.A. and D.W. Griffin, 2006: Aerobiology and the global transport of desert dust. Trends in Ecology \& Evolution, 21(11), 638-644.

Kendon, E.J., D.P. Rowell, R.G. Jones, and E. Buonomo, 2008: Robustness of future changes in local precipitation extremes. Journal of Climate, 21(17), 4280-4297.

Kendon, E.J., D.P. Rowell, and R.G. Jones, 2010: Mechanisms and reliability of future projected changes in daily precipitation. Climate Dynamics, 35(2-3), 489-509.

Kenyon, J., and G.C. Hegerl, 2008: Influence of modes of climate variability on global temperature extremes. Journal of Climate, 21(15), 3872-3889.

Kershaw, J.A., J.J. Clague, and S.G. Evans, 2005: Geomorphic and sedimentological signature of a two-phase outburst flood from moraine-dammed Queen Bess Lake, British Columbia, Canada. Earth Surface Processes and Landforms, 30(1), $1-25$.

Kharin, V., F.W. Zwiers, X. Zhang, and G.C. Hegerl, 2007: Changes in temperature and precipitation extremes in the IPCC ensemble of global coupled model simulations. Journal of Climate, 20(8), 1419-1444.

Khon, V.C., I.I. Mokhov, E. Roeckner, and V.A. Semenov, 2007: Regional changes of precipitation characteristics in Northern Eurasia from simulations with global climate model. Global and Planetary Change, 57(1-2), 118-123.

Kidston, J., and E.P. Gerber, 2010: Intermodel variability of the poleward shift of the austral jet stream in the CMIP3 integrations linked to biases in 20th century climatology. Geophysical Research Letters, 37(9), L09708.

Kiktev, D., D.M.H. Sexton, L. Alexander, and C.K. Folland, 2003: Comparison of modeled and observed trends in indices of daily climate extremes. Journal of Climate, 16(22), 3560-3571.

Kiktev, D.M., J. Caesar, and L. Alexander, 2009: Temperature and precipitation extremes in the second half of the twentieth century from numerical modeling results and observational data. Izvestiya Atmospheric and Oceanic Physics, 45, 284-293.

Kilsby, C., P. Jones, A. Burton, A. Ford, H. Fowler, C. Harpham, P. James, A. Smith, and R. Wilby, 2007: A daily weather generator for use in climate change studies. Environmental Modelling and Software, 22(12), 1705-1719.

Kim, H.J., R.C. Sidle, R.D. Moore, and R. Hudson, 2004: Throughflow variability during snowmelt in a forested mountain catchment, coastal British Columbia, Canada. Hydrological Processes, 18(7), 1219-1236.

Kim, H.J., B. Wang, and Q.H. Ding, 2008: The global monsoon variability simulated by CMIP3 coupled climate models. Journal of Climate, 21(20), 5271-5294.

Kim, S., E. Nakakita, Y. Tachikawa, and K. Takara, 2010: Precipitation changes in Japan under the A1B climate change scenario. Annual Journal of Hydraulic Engineering, JSCE, 54, 127-132.

Kim, S.K., W.P. Hong, and Y.M. Kim, 1991: Prediction of rainfall-triggered landslides in Korea. In: Proceedings of the 6th International Symposium on Landslides, Christchurch, New Zealand [Bell, D.H. (ed.)]. Balkema, Rotterdam, The Netherlands, pp. 989-994.

Kimoto, M., N. Yasutomi, C. Yokoyama, and S. Emori, 2005: Projected changes in precipitation characteristics around Japan under the global warming. SOLA, 1, 85-88

King'uyu, S.M., L.A. Ogallo, and E.K. Anyamba, 2000: Recent trends of minimum and maximum surface temperatures over eastern Africa. Journal of Climate, 13(16), 2876-2886. 
Kitoh, A. and T. Uchiyama, 2006: Changes in onset and withdrawal of the East Asian summer rainy season by multi-model global warming experiments. Journal of the Meteorological Society of Japan, 84(2), 247-258.

Kitoh, A., T. Ose, K. Kurihara, S. Kusunoki, M. Sugi, and KAKUSHIN Team-3 Modeling Group, 2009: Projection of changes in future weather extremes using superhigh-resolution global and regional atmospheric models in the KAKUSHIN Program: Results of preliminary experiments. Hydrological Research Letters, 3, 49-53.

Kitoh, A., S. Kusunoki, and T. Nakaegawa, 2011: Climate change projections over South America in the late 21st century with the 20 and $60 \mathrm{~km}$ mesh Meteorological Research Institute atmospheric general circulation model (MRIAGCM). Journal of Geophysical Research - Atmospheres, 116, D06105.

Kjellström, E. and P. Lind, 2009: Changes in the water budget in the Baltic Sea drainage basin in future warmer climates as simulated by the regional climate model RCA3. Boreal Environment Research, 14(1), 114-124.

Kjellström, E., L. Bärring, D. Jacob, R. Jones, G. Lenderink, and C. Schär, 2007: Modelling daily temperature extremes: recent climate and future changes over Europe. Climatic Change, 81(S1), 249-265.

Klein Tank, A.M.G., and G.P. Können, 2003: Trends in indices of daily temperature and precipitation extremes in Europe, 1946-1999. Journal of Climate, 16(22), 3665-3680.

Klein Tank, A.M.G., T.C. Peterson, D.A. Quadir, S. Dorji, X. Zou, H. Tang, K. Santhosh, U.R. Joshi, A.K. Jaswal, R.K. Kolli, A.B. Sikder, N.R. Deshpande, J.V. Revadekar, K. Yeleuova, S. Vandasheva, M. Faleyeva, P. Gomboluudev, K.P. Budhathoki, A. Hussain, M. Afzaal, L. Chandrapala, H. Anvar, D. Amanmurad, V.S. Asanova, P.D. Jones, M.G. New, and T. Specktorman, 2006: Changes in daily temperature and precipitation extremes in central and south Asia. Journal of Geophysical Research, 111, D16105.

Knapp, K.R. and J.P. Kossin, 2007: A new global tropical cyclone data set from ISCCP B1 geostationary satellite observations. Journal of Applied Remote Sensing, 1, 013505.

Knapp, K.R. and M.C. Kruk, 2010: Quantifying inter-agency differences in tropical cyclone best track wind speed estimates. Monthly Weather Review, 138(4), 1459-1473.

Kniveton, D.R., R. Layberry, C.J.R. Williams, and M. Peck, 2009: Trends in the start of the wet season over Africa. International Journal of Climatology, 29(9), 1216-1225.

Knowles, N., M.D. Dettinger, and D.R. Cayan, 2006: Trends in snowfall versus rainfall in the western United States. Journal of Climate, 19(18), 4545-4559.

Knox, J.C., 2000: Sensitivity of modern and Holocene floods to climate change. Quaternary Science Reviews, 19(1-5), 439-457.

Knutson, T.R. and R.E. Tuleya, 2004: Impact of $\mathrm{CO}_{2}$-induced warming on simulated hurricane intensity and precipitation: Sensitivity to the choice of climate model and convective parameterization. Journal of Climate, 17(18), 3477-3495.

Knutson, T.R., T.L. Delworth, K.W. Dixon, I.M. Held, J. Lu, V. Ramaswamy, M.D. Schwarzkopf, G. Stenchikov, and R.J. Stouffer, 2006: Assessment of twentiethcentury regional surface temperature trends using the GFDL CM2 coupled models. Journal of Climate, 19(9), 1624-1651.

Knutson, T.R., J.J. Sirutis, S.T. Garner, I.M. Held, and R.E. Tuleya, 2007: Simulation of the recent multidecadal increase of Atlantic hurricane activity using an $18-\mathrm{km}$ grid regional model. Bulletin of the American Meteorological Society, 88(10), 1549-1565.

Knutson, T.R., J.J. Sirutis, S.T. Garner, G.A. Vecchi, and I.M. Held, 2008: Simulated reduction in Atlantic hurricane frequency under twenty-first- century warming conditions. Nature Geoscience, 1(6), 359-364.

Knutson, T.R., J.L. McBride, J. Chan, K. Emanuel, G. Holland, C. Landsea, I. Held, J.P. Kossin, A.K. Srivastava, and M. Sugi, 2010: Tropical cyclones and climate change. Nature Geoscience, 3(3), 157-163.

Knutti, R., 2010: The end of model democracy? Climatic Change, 102(3-4), 395-404.

Knutti, R., R. Furrer, C. Tebaldi, J. Cermak, and G.A. Meehl, 2010a: Challenges in combining projections from multiple climate models. Journal of Climate, 23(10), 2739-2758
Knutti, R., G. Abramowitz, M. Collins, V. Eyring, P.J. Glecker, B. Hewitson, and L. Mearns, 2010b: Good practice guidance paper on assessing and combining multi model climate projections. In: Meeting Report of the Intergovernmental Panel on Climate Change Expert Meeting on Assessing and Combining MultiModel Climate Projections [Stocker, T.F., Q. Dahe, G.-K. Plattner, M. Tignor, and P.M. Midgley (eds.)]. IPCC Working Group I Technical Support Unit, University of Bern, Bern, Switzerland, pp. 1-13.

Kochel, R.C., and V.R. Baker, 1982: Paleoflood hydrology. Science, 215(4531), 353-361.

Kodama, C. and T. Iwasaki, 2009: Influence of the SST rise on baroclinic instability wave activity under an aquaplanet condition. Journal of the Atmospheric Sciences, 66(8), 2272-2287.

Koffi, B. and E. Koffi, 2008: Heat waves across Europe by the end of the 21st century: multiregional climate simulations. Climate Research, 36(2), 153-168.

Komar, P.D. and J.C. Allan, 2008: Increasing hurricane-generated wave heights along the US East Coast and their climate controls. Journal of Coastal Research, 24(2), 479-488.

Kont, A., J. Jaagus, R. Aunap, U. Ratas, and R. Rivis, 2008: Implications of sea-level rise for Estonia. Journal of Coastal Research, 24(2), 423-431.

Koo, G.S., K.O. Boo, and W.T. Kwon, 2009: Projection of temperature over Korea using an MM5 regional climate simulation. Climate Research, 40(2-3), 241-248.

Kossin, J.P., 2008: Is the North Atlantic hurricane season getting longer? Geophysical Research Letters, 35, L23705.

Kossin, J.P. and D.J. Vimont, 2007: A more general framework for understanding Atlantic hurricane variability and trends. Bulletin of the American Meteorology Society, 88(11), 1767-1781.

Kossin, J.P., K.R. Knapp, D.J. Vimont, R.J. Murnane, and B.A. Harper, 2007: A globally consistent reanalysis of hurricane variability and trends. Geophysical Research Letters, 34, L04815.

Kossin, J.P., S.J. Camargo, and M. Sitkowski, 2010: Climate modulation of North Atlantic hurricane tracks. Journal of Climate, 23(11), 3057-3076.

Koster, R.D. and M.J. Suarez, 2001: Soil moisture memory in climate models. Journal of Hydrometeorology, 2(6), 558-570.

Koster, R.D., M.J. Suarez, P. Liu, U. Jambor, A. Berg, M. Kistler, R. Reichle, M. Rodell, and J. Famiglietti, 2004a: Realistic initialization of land surface states: Impacts on subseasonal forecast skill. Journal of Hydrometeorology, 5(6), 1049-1063.

Koster, R.D., P.A. Dirmeyer, Z.C. Guo, G. Bonan, E. Chan, P. Cox, C.T. Gordon, S. Kanae, E. Kowalczyk, D. Lawrence, P. Liu, C.H. Lu, S. Malyshev, B. McAvaney, K. Mitchell, D. Mocko, T. Oki, K. Oleson, A. Pitman, Y.C. Sud, C.M. Taylor, D. Verseghy, R. Vasic, Y.K. Xue, and T. Yamada, 2004b: Regions of strong coupling between soil moisture and precipitation. Science, 305, 1138-1140.

Koster, R.D., Z.C. Guo, R.Q. Yang, P.A. Dirmeyer, K. Mitchell, and M.J. Puma, 2009: On the nature of soil moisture in land surface models. Journal of Climate, 22(16), 4322-4335.

Kotlyakov, V.M., O.V. Rototaeva, and G.A. Nosenko, 2004: The September 2002 Kolka glacier catastrophe in North Ossetia, Russian Federation: evidence and analysis. Mountain Research and Development, 24(1), 78-83.

Kripalani, R.H., J.H. Oh, A. Kulkarni, S.S. Sabade, and H.S. Chaudhari, 2007: South Asian summer monsoon precipitation variability: Coupled climate model simulations and projections under IPCC AR4. Theoretical and Applied Climatology, 90(3-4), 133-159.

Krishnamurthy, C.K.B., U. Lall, and H.H. Kwon, 2009: Changing frequency and intensity of rainfall extremes over India from 1951 to 2003. Journal of Climate, 22(18), 4737-4746.

Kruger, A.C., 2006: Observed trends in daily precipitation indices in South Africa: 1910-2004. International Journal of Climatology, 26(15), 2275-2285.

Kubota, H. and J.C.L. Chan, 2009: Interdecadal variability of tropical cyclone landfall in the Philippines from 1902 to 2005. Geophysical Research Letters, 36, L12802.

Kürbis, K., M. Mudelsee, G. Tetzlaff, and R. Brazdil, 2009: Trends in extremes of temperature, dewpoint, and precipitation from long instrumental series from central Europe. Theoretical and Applied Climatology, 98(1-2), 187-195.

Kuglitsch, F.G., A. Toreti, E. Xoplaki, P.M. Della-Marta, J. Luterbacher, and H. Wanner, 2009: Homogenization of daily maximum temperature series in the Mediterranean. Journal of Geophysical Research - Atmospheres, 114, D15108. 
Kuglitsch, F.G., A. Toreti, E. Xoplaki, P.M. Della-Marta, C. Zerefos, M. Türkes, and J. Luterbacher, 2010: Heat wave changes in the eastern Mediterranean since 1960. Geophysical Research Letters, 37, L04802.

Kuleshov, Y., L. Qi, R. Fawcett, and D. Jones, 2008: On tropical cyclone activity in the Southern Hemisphere: Trends and the ENSO connection. Geophysical Research Letters, 35, L14S08.

Kumar, K.R., A.K. Sahai, K.K. Kumar, S.K. Patwardhan, P.K. Mishra, J.V. Revadekar, K. Kamala, and G.B. Pant, 2006: High-resolution climate change scenarios for India for the 21st century. Current Science, 90(3), 334-345.

Kundzewicz, Z.W., L.J. Mata, N.W. Arnell, P. Doll, P. Kabat, B. Jimenez, K.A. Miller, T. Oki, Z. Sen, and I.A. Shiklomanov, 2007: Freshwater resources and their management. In: Climate Change 2007. Impacts, Adaptation and Vulnerability. Contribution of Working Group II to the Fourth Assessment Report of the Intergovernmental Panel on Climate Change [Parry, M.L., O.F. Canziani, J.P. Palutikof, P.J. Van Der Linde, and C.E. Hanson (eds.)]. Cambridge University Press, Cambridge, UK, pp. 173-210.

Kunkel, K.E., P.D. Bromirski, H.E. Brooks, T. Cavazos, A.V. Douglas, D.R. Easterling, K.A. Emanuel, P.Y. Groisman, G.J. Holland, T.R. Knutson, J.P. Kossin, P.D. Komar, D.H. Levinson, and R.L. Smith, 2008: Observed changes in weather and climate extremes. In: Weather and Climate Extremes in a Changing Climate. Regions of Focus: North America, Hawaii, Caribbean, and U.S. Pacific Islands. [Karl, T.R. G.A. Meehl, D.M. Christopher, S.J. Hassol, A.M. Waple, and W.L. Murray (eds.)]. A Report by the U.S. Climate Change Science Program and the Subcommittee on Global Change Research, Washington, DC, pp. 222.

Kunkel, K.E., X.-Z. Liang, and J. Zhu, 2010: Regional climate model projections and uncertainties of U.S. summer heat waves. Journal of Climate, 23(16), 4447-4458.

Kunz, M., J. Sander, and C. Kottmeier, 2009: Recent trends of thunderstorm and hailstorm frequency and their relation to atmospheric characteristics in southwest Germany. International Journal of Climatology, 29(15), 2283-2297.

Kusunoki, S. and R. Mizuta, 2008: Future changes in the Baiu rain band projected by a 20-km mesh global atmospheric model: sea surface temperature dependence. SOLA, 4, 85-88.

Kwarteng, A.Y., A.S. Dorvlo, and G.T.V. Kumar, 2009: Analysis of a 27-year rainfall data (1977-2003) in the Sultanate of Oman. International Journal of Climatology, 29(4), 605-617.

Kyselý, J., 2009: Trends in heavy precipitation in the Czech Republic over 1961-2005. International Journal of Climatology, 29(12), 1745-1758.

Kyselý, J. and R. Beranová, 2009: Climate-change effects on extreme precipitation in central Europe: uncertainties of scenarios based on regional climate models. Theoretical and Applied Climatology, 95(3-4), 361-374.

Laine, A., M. Kageyama, D. Salas-Melia, G. Ramstein, S. Planton, S. Denvil, and S. Tyteca, 2009: An energetics study of wintertime Northern Hemisphere storm tracks under $4 \times \mathrm{CO}_{2}$ conditions in two ocean-atmosphere coupled models. Journal of Climate, 22(3), 819-839.

Lambeck, K., C.D. Woodroffe, F. Antonioli, M. Anzidei, W.R. Gehrels, J. Laborel, and A.J. Wright, 2010: Paleoenvironmental records, geophysical modeling, and reconstruction of sea-level trends and variability on centennial and longer timescales. In: Understanding Sea-Level Rise and Variability [Church, J.A., P.L. Woodworth, T. Aarup, and W.S. Wilson (eds.)]. Wiley-Blackwell, Chichester, UK, pp. 61-121.

Landsea, C.W., 1993: A climatology of intense (or major) Atlantic hurricanes. Monthly Weather Review, 121(6), 1703-1713.

Landsea, C.W., 2007: Counting Atlantic tropical cyclones back to 1900. EOS Transactions (AGU), 88(18), 197-202.

Landsea, C.W., C. Anderson, N. Charles, G. Clark, J. Dunion, J. Fernandez-Partagas, P. Hungerford, C. Neumann, and M. Zimmer, 2004: The Atlantic hurricane database re-analysis project: Documentation for the 1851-1910 alterations and additions to the HURDAT database. In: Hurricanes and Typhoons: Past, Present and Future [Murnane, R.J. and K.B. Liu (eds.)]. Columbia University Press, New York, NY, pp. 177-221.

Landsea, C.W., B.A. Harper, K. Hoarau, and J.A. Knaff, 2006: Can we detect trends in extreme tropical cyclones? Science, 313(5786), 452-454.
Lantz, T.C., and S.V. Kokelj, 2008: Increasing rates of retrogressive thaw slump activity in the Mackenzie Delta region, N.W.T., Canada. Geophysical Research Letters, 35, L06502.

Laprise, R., R. de Elia, D. Caya, S. Biner, P. Lucas-Picher, E. Diaconescu, M. Leduc, A. Alexandru, L. Separovic, and C. Canadian Network Regional, 2008: Challenging some tenets of regional climate modelling. Meteorology and Atmospheric Physics, 100(1-4), 3-22.

Larsen, C.F., R.J. Motyka, A.A. Arendt, K.A. Echelmeyer, and P.E. Geissler, 2007: Glacier changes in southeast Alaska and northwest British Columbia and contribution to sea level rise. Journal of Geophysical Research, 112, F01007.

Lau, K.M. and H.T. Wu, 2007: Detecting trends in tropical rainfall characteristics, 1973-2003. International Journal of Climatology, 27(8), 979-988.

Lau, K.M., M.K. Kim, and K.M. Kim, 2006: Asian summer monsoon anomalies induced by aerosol direct forcing: the role of the Tibetan Plateau. Climate Dynamics, 26(7-8), 855-864.

Lau, K.M., Y.P. Zhou, and H.T. Wu, 2008a: Have tropical cyclones been feeding more extreme rainfall? Journal of Geophysical Research, 113, D23113.

Lau, N.C., A. Leetma, and M.J. Nath, 2008b: Interactions between the responses of North American climate to El Niño-La Niña and to the secular warming trend in the Indian-Western Pacific Oceans. Journal of Climate, 21(3), 476-494.

Laurent, C. and S. Parey, 2007: Estimation of 100-year return-period temperatures in France in a non-stationary climate: Results from observations and IPCC scenarios. Global and Planetary Change, 57(1-2), 177-188.

Le Cozannet, G., S. Lecacheux, E. Delvallee, N. Desramaut, C. Oliveros, and R. Pedreros, 2011: Teleconnection pattern influence on sea-wave climate in the Bay of Biscay. Journal of Climate, 24(3), 641-652.

Le Quesne, C., D.W. Stahle, M.K. Cleaveland, M.D. Therrell, J.C. Aravena, and J. Barichivich, 2006: Ancient Austrocedrus tree-ring chronologies used to reconstruct central Chile precipitation variability from A.D. 1200 to 2000 . Journal of Climate, 19(22), 5731-5744.

Le Quesne, C., C. Acuna, J.A. Boninsegna, A. Rivera, and J. Barichivich, 2009: Longterm glacier variations in the Central Andes of Argentina and Chile, inferred from historical records and tree-ring reconstructed precipitation. Palaeogeography, Palaeoclimatology, Palaeoecology, 281(3-4), 334-344.

Leake, J., J. Wolf, J. Lowe, P. Stansby, G. Jacoub, R. Nicholls, M. Mokrech, S. NicholsonCole, M. Walkden, A. Watkinson, and S. Hanson, 2007: Predicted wave climate for the UK: towards an integrated model of coastal impacts of climate change. In: Estuarine and Coastal Modeling Congress 2007 [Spaulding, M. (ed.)] Proceedings of the Tenth International Conference on Estuarine and Coastal Modeling Congress 2007, Newport, Rhode Island, 5-7 Nov 2007, American Society of Civil Engineers, pp 393-406.

Leander, R., T.A. Buishand, B.J.J.M. van den Hurk, and M.J.M. de Wit, 2008: Estimated changes in flood quantiles of the river Meuse from resampling of regional climate model output. Journal of Hydrology, 351(3-4), 331-343.

Leckebusch, G.C., B. Koffi, U. Ulbrich, J.G. Pinto, T. Spangehl, and S. Zacharias, 2006: Analysis of frequency and intensity of European winter storm events from a multi-model perspective, at synoptic and regional scales. Climate Research, 31(1), 59-74.

Leckebusch, G.C., D. Renggli, and U. Ulbrich, 2008: Development and application of an objective storm severity measure for the Northeast Atlantic region. Meteorologische Zeitschrift, 17(5), 575-587.

Leclercq, P., J. Oerlemans, and J. Cogley, 2011: Estimating the glacier contribution to sea-level rise for the period 1800-2005. Surveys in Geophysics, 32(4-5), 519-535, doi:10.1007/s10712-10011-19121-10717.

Lee, T. and M.J. McPhaden, 2010: Increasing intensity of El Niño in the centralequatorial Pacific. Geophysical Research Letters, 37, L14603.

Legates, D.R., H.F. Lins, and G.J. McCabe, 2005: Comments on "Evidence for global runoff increase related to climate warming" by Labat et al. Advances in Water Resources, 28(12), 1310-1315.

Lehmann, A., K. Getzlaff, and J. Harlass, 2011: Detailed assessment of climate variability in the Baltic Sea area for the period 1958 to 2009. Climate Research, 46(2), 185-196 
Lehner, B., P. Doll, J. Alcamo, T. Henrichs, and F. Kaspar, 2006: Estimating the impact of global change on flood and drought risks in Europe: A continental, integrated analysis. Climatic Change, 75(3), 273-299.

Leloup, J., M. Lengaigne, and J.-P. Boulanger, 2008: Twentieth century ENSO characteristics in the IPCC database. Climate Dynamics, 30(2-3), 227-291.

Lemke, P., J. Ren, R.B. Alley, I. Allison, J. Carrasco, G. Flato, Y. Fujii, G. Kaser, P. Mote, R.H. Thomas, and T. Zhang, 2007: Observations: Changes in snow, ice and frozen ground. In: Climate Change 2007: The Physical Science Basis. Contribution of Working Group I to the Fourth Assessment Report of the Intergovernmental Panel on Climate Change [Solomon, S., D. Qin, M. Manning, Z. Chen, M. Marquis, K.B. Averyt, M. Tignor and H.L. Miller (eds.)]. Cambridge University Press, Cambridge, UK, and New York, NY, pp. 337-383.

Lenderink, G. and E. Van Meijgaard, 2008: Increase in hourly precipitation extremes beyond expectations from temperature changes. Nature Geoscience, 1(8), 511-514.

Lenderink, G. and E. van Meijgaard, 2009: Unexpected rise in extreme precipitation caused by a shift in rain type? Nature Geoscience, 2(6), 373-373.

Lenton, T.M., H. Held, E. Kriegler, J.W. Hall, W. Lucht, S. Rahmstorf, and H.J. Schellnhuber, 2008: Tipping elements in the Earth's climate system. Proceedings of the National Academy of Sciences, 105(6), 1786-1793.

Leslie, L.M., M. Leplastrier, and B.W. Buckley, 2008: Estimating future trends in severe hailstorms over the Sydney Basin: A climate modelling study. Atmospheric Research, 87(1), 37-57.

Lewis, S.L., P.M. Brando, O.L. Phillips, G.M.F. van der Heijden, and D. Nepstad, 2011: The 2010 Amazon Drought. Science, 331(6017), 554.

Lewkowicz, A.G. and C. Harris, 2005: Morphology and geotechnique of active-layer detachment failures in discontinuous and continuous permafrost, northern Canada. Geomorphology, 69(1-4), 275-297.

L'Hôte, Y., G. Mahé, B. Somé, and J.P. Triboulet, 2002: Analysis of a Sahelian annual rainfall index from 1896 to 2000; the drought continues. Hydrological Sciences Journal, 47(4), 563-572.

Li, H., A. Dai, T. Zhou, and J. Lu, 2008: Responses of East Asian summer monsoon to historical SST and atmospheric forcing during 1950-2000. Climate Dynamics, 34(4), 501-514.

Li, X.Y., L.Y. Liu, and J.H. Wang, 2004: Wind tunnel simulation of aeolian sandy soil erodibility under human disturbance. Geomorphology, 59(1-4), 3-11.

Liggins, F., R.A. Betts, and B. McGuire, 2010: Projected future climate changes in the context of geological and geomorphological hazards. Philosophical Transactions of the Royal Society A: Mathematical, Physical and Engineering Sciences, 368(1919), 2347-2367.

Lim, E.-P., and I. Simmonds, 2009: Effect of tropospheric temperature change on the zonal mean circulation and SH winter extratropical cyclones. Climate Dynamics, 33(1), 19-32.

Lin, J.L., B.E. Mapes, K.M. Weickmann, G.N. Kiladis, S.D. Schubert, M.J. Suarez, J.T. Bacmeister, and M.I. Lee, 2008a: North American monsoon and convectively coupled equatorial waves simulated by IPCC AR4 coupled GCMs. Journal of Climate, 21(12), 2919-2937.

Lin, J.L., K.M. Weickman, G.N. Kiladis, B.E. Mapes, S.D. Schubert, M.J. Suarez, J.T. Bacmeister, and M.I. Lee, 2008b: Subseasonal variability associated with Asian summer monsoon simulated by 14 IPCC AR4 coupled GCMs. Journal of Climate, 21(18), 4541-4567.

Lindström, G. and S. Bergström, 2004: Runoff trends in Sweden 1807-2002. Hydrological Sciences Journal, 49(1), 69-83.

Lins, H.F. and J.R. Slack, 1999: Streamflow trends in the United States. Geophysical Research Letters, 26(2), 227-230.

Lionello, P., S. Cogo, M.B. Galati, and A. Sanna, 2008: The Mediterranean surface wave climate inferred from future scenario simulations. Global and Planetary Change, 63(2-3), 152-162.

Lipovsky, P., S. Evans, J. Clague, C. Hopkinson, R. Couture, P. Bobrowsky, G. Ekström, M. Demuth, K. Delaney, N. Roberts, G. Clarke, and A. Schaeffer, 2008: The July 2007 rock and ice avalanches at Mount Steele, St. Elias Mountains, Yukon, Canada. Landslides, 5(4), 445-455.
Liu, B., M. Xu, and M. Henderson, 2011: Where have all the showers gone? Regional declines in light precipitation events in China, 1960-2000. International Journal of Climatology, 31(8), 1177-1191.

Llano, M.P. and O. Penalba, 2011: A climatic analysis of dry sequences in Argentina. International Journal of Climatology, 31(4), 504-513.

Llasat, M.C., M. Barriendos, A. Barrera, and T. Rigo, 2005: Floods in Catalonia (NE Spain) since the 14th century. Climatological and meteorological aspects from historical documentary sources and old instrumental records. Journal of Hydrology, 313(1-2), 32-47.

Lloyd-Hughes, B., and M.D. Saunders, 2002: A drought climatology for Europe. International Journal of Climatology, 22(13), 1571-1592.

Lobell, D.B., C.J. Bonfils, L.M. Kueppers, and M.A. Snyder, 2008: Irrigation cooling effect on temperature and heat index extremes. Geophysical Research Letters, 35, L09705.

Loeptien, U., O. Zolina, S. Gulev, M. Latif, and V. Soloviov, 2008: Cyclone life cycle characteristics over the Northern Hemisphere in coupled GCMs. Climate Dynamics, 31(5), 507-532.

Lohmann, U. and J. Feichter, 2007: Global indirect aerosol effects: a review. Atmospheric Chemistry and Physics, 5(3), 715-737.

Long, Z., W. Perrie, J. Gyakum, R. Laprise, and D. Caya, 2009: Scenario changes in the climatology of winter midlatitude cyclone activity over eastern North America and the Northwest Atlantic. Journal of Geophysical Research - Atmospheres, 114, D12111.

Lorenz, D.J., and E.T. DeWeaver, 2007: Tropopause height and zonal wind response to global warming in the IPCC scenario integrations. Journal of Geophysical Research-Atmospheres, 112, D10119.

Lorenz, D.J. and D.L. Hartmann, 2003: Eddy-zonal flow feedback in the Northern Hemisphere winter. Journal of Climate, 16(8), 1212-1227.

Lorenz, R., E.B. Jaeger, and S.I. Seneviratne, 2010: Persistence of heat waves and its link to soil moisture memory. Geophysical Research Letters, 37, L09703.

Lowe, J.A., P.L. Woodworth, T. Knutson, R.E. McDonald, K.L. McInnes, K. Woth, H. von Storch, J. Wolf, V. Swail, N.B. Bernier, S. Gulev, K.J. Horsburgh, A.S. Unnikrishnan, J.R. Hunter, and R. Weisse, 2010: Past and future changes in extreme sea levels and waves. In: Understanding Sea-Level Rise and Variability [Church, J.A., P.L. Woodworth, T. Aarup, and W.S. Wilson (eds.)]. Wiley-Blackwell, Chichester, UK, pp. 326-375.

Lu, J., G.A. Vecchi, and T. Reichler, 2007: Expansion of the Hadley cell under global warming. Geophysical Research Letters, 34, L06805.

Lugon, R. and M. Stoffel, 2010: Rock-glacier dynamics and magnitude-frequency relations of debris flows in a high-elevation watershed: Ritigraben, Swiss Alps. Global and Planetary Change, 73(3-4), 202-210.

Lupikasza, E., 2010: Spatial and temporal variability of extreme precipitation in Poland in the period 1951-2006. International Journal of Climatology, 30(7), 991-1007.

Luterbacher, J., D. Dietrich, E. Xoplaki, M. Grosjean, and H. Wanner, 2004: European seasonal and annual temperature variability, trends, and extremes since 1500 . Science, 303(5663 ), 1499-1503.

Lynch, A.H., J.A. Curry, R.D. Brunner, and J.A. Maslanik, 2004: Toward an integrated assessment of the impacts of extreme wind events on Barrow, Alaska. Bulletin of the American Meteorological Society, 85(2), 209-221.

Mabuchi, K., Y. Sato, and H. Kida, 2005a: Climatic impact of vegetation change in the Asian tropical region. Part I: Case of the Northern Hemisphere summer. Journal of Climate, 18(3), 410-428.

Mabuchi, K., Y. Sato, and H. Kida, 2005b: Climatic impact of vegetation change in the Asian tropical region. Part II: Case of the Northern Hemisphere winter and impact on the extratropical circulation. Journal of Climate, 18(3), 429-446.

Mann, M.E. and K.A. Emanuel, 2006: Atlantic hurricane trends linked to climate change. Eos Transactions (AGU), 87(24), 233-241.

Mann, M.E., K.A. Emanuel, G.J. Holland, and P.J. Webster, 2007a: Atlantic tropical cyclones revisited. Eos Transactions (AGU), 88, 349-350.

Mann, M.E., T.A. Sabbatelli, and U. Neu, 2007b: Evidence for a modest undercount bias in early historical Atlantic tropical cyclone counts. Geophysical Research Letters, 34, L22707. 
Mann, M.E., J.D. Woodruff, J.P. Donnelly, and Z. Zhang, 2009: Atlantic hurricanes and climate over the past 1,500 years. Nature, 460, 880-883.

Manson, G.K., and S.M. Solomon, 2007: Past and future forcing of Beaufort Sea coastal change. Atmosphere-Ocean, 45(2), 107-122.

Manton, M.J., P.M. Della-Marta, M.R. Haylock, K.J. Hennessy, N. Nicholls, L.E. Chambers, D.A. Collins, G. Daw, A. Finet, D. Gunawan, K. Inape, H. Isobe, T.S. Kestin, P. Lefale, C.H. Leyu, T. Lwin, L. Maitrepierre, N. Ouprasitwong, C.M. Page, J. Pahalad, N. Plummer, M.J. Salinger, R. Suppiah, V.L. Tran, B. Trewin, I. Tibig, and D. Yee, 2001: Trends in extreme daily rainfall and temperature in Southeast Asia and the South Pacific: 1961-1998. International Journal of Climatology, 21(3), 269-284.

Maraun, D., T.J. Osborn, and N.P. Gillet, 2008: United Kingdom daily precipitation intensity: improved early data, error estimates and an update from 2000 to 2006. International Journal of Climatology, 28(6), 833-842.

Maraun, D., F. Wetterhall, A.M. Ireson, R.E. Chandler, E.J. Kendon, M. Widmann, S. Brienen, H.W. Rust, T. Sauter, M. Themessl, V.K.C. Venema, K.P. Chun, C.M. Goodess, R.G. Jones, C. Onof, M. Vrac, and I. Thiele-Eich, 2010: Precipitation downscaling under climate change. Recent developments to bridge the gap between dynamical models and the end user. Reviews of Geophysics, 48, RG3003.

Marcos, M., M.N. Tsimplis, and A.G.P. Shaw, 2009: Sea level extremes in southern Europe. Journal of Geophysical Research - Oceans, 114, C01007.

Marengo, J.A., R. Jones, L.M. Alves, and M.C. Valverde, 2009a: Future change of temperature and precipitation extremes in South America as derived from the PRECIS regional climate modeling system. International Journal of Climatology, 29(15), 2241-2255.

Marengo, J.A., M. Rusticucci, O. Penalba, and M. Renom, 2009b: An intercomparison of observed and simulated extreme rainfall and temperature events during the last half of the twentieth century: part 2: historical trends. Climatic Change, 98(3-4), 509-529.

Marengo, J.A., J. Tomasella, W. Soares, L. Alves, and C. Nobre, 2011: Extreme climatic events in the Amazon basin. Theoretical and Applied Climatology, doi:10.1007/s00704-00011-00465-00701.

Mariotti, A., N. Zeng, J.H. Yoon, V. Artale, A. Navarra, P. Alpert, and L.Z.X. Li, 2008: Mediterranean water cycle changes: transition to drier 21st century conditions in observations and CMIP3 simulations. Environmental Research Letters, 3(4), 044001.

Marques, C., A. Rocha, and J. Corte-Real, 2011: Global diagnostic energetics of five state-of-the-art climate models. Climate Dynamics, 36(9-10), 1767-1794.

Martin, J.H., and R.M. Gordon, 1988: Northeast Pacific iron distributions in relation to phytoplankton productivity. Deep-Sea Research Part A - Oceanographic Research Papers, 35(2), 177-196.

Mastin, L.G., 1994: Explosive tephra emissions at Mount St. Helens, 1989-1991: The violent escape of magmatic gas following storms? Geological Society of America Bulletin, 106(2), 175-185.

Mastrandrea, M.D., C.B. Field, T.F. Stocker, O. Edenhofer, K.L. Ebi, D.J. Frame, H. Held, E. Kriegler, K.J. Mach, P.R. Matschoss, G.-K. Plattner, G.W. Yohe, and F.W. Zwiers, 2010: Guidance Note for Lead Authors of the IPCC Fifth Assessment Report on Consistent Treatment of Uncertainties. Intergovernmental Panel on Climate Change (IPCC), Geneva, Switzerland.

Matthews, A.J., J. Barclay, S. Carn, G. Thompson, J. Alexander, R. Herd, and C. Williams, 2002: Rainfall-induced volcanic activity on Montserrat. Geophysical Research Letters, 29(13), 1644.

Matulla, C., W. Schoener, H. Alexandersson, H. von Storch, and X.L. Wang, 2008: European storminess: late nineteenth century to present. Climate Dynamics, 31(2-3), 125-130.

Maue, R.N., 2009: Northern Hemisphere tropical cyclone activity. Geophysical Research Letters, 36, L05805.

May, W., 2008: Potential future changes in the characteristics of daily precipitation in Europe simulated by the HIRHAM regional climate model. Climate Dynamics, 30(6), 581-603.

McCabe, G.J., and D.M. Wolock, 2002: A step increase in streamflow in the conterminous United States. Geophysical Research Letters, 29(24), 2185.
McDonald, R., 2011: Understanding the impact of climate change on Northern Hemisphere extra-tropical cyclones. Climate Dynamics, 37(7-8), 1399-1425, doi:10.1007/s00382-00010-00916-x.

McGranahan, G., D. Balk, and B. Anderson, 2007: The rising tide: assessing the risks of climate change and human settlements in low elevation coastal zones. Environment and Urbanization, 19(1), 17-37.

McGuire, B., 2010: Potential for a hazardous geospheric response to projected future climate changes. Philosophical Transactions of the Royal Society A, 368(1919), 2317-2345.

McInnes, K.L., K.J.E. Walsh, G.D. Hubbert, and T. Beer, 2003: Impact of sea-level rise and storm surges on a coastal community. Natural Hazards, 30(2), 187-207.

McInnes, K.L., I. Macadam, and J.G. O'Grady, 2009a: The Effect of Climate Change on Extreme Sea Levels along Victoria's Coast. A Project Undertaken for the Department of Sustainability and Environment, Victoria as part of the 'Future Coasts' Program. CSIRO, Victoria, Australia, 55 pp.

McInnes, K.L., I. Macadam, G.D. Hubbert, and J.G. O'Grady, 2009b: A modelling approach for estimating the frequency of sea level extremes and the impact of climate change in southeast Australia. Natural Hazards, 51(1), 115-137.

McInnes, K.L., T.A. Erwin, and J.M. Bathols, 2011: Global Climate Model projected changes in $10 \mathrm{~m}$ wind speed and direction due to anthropogenic climate change. Atmospheric Science Letters, doi:10.1002/asl.1341.

McKee, T.B., N.J. Doesken, and J. Kleist, 1993: The relationship of drought frequency and duration to time scales. In: Proceedings of the 8th Conference on Applied Climatology, Anaheim, California, 17-22 Jan 1993, pp. 179-184.

McKendry, I.G., K.B. Strawbridge, N.T. O'Neill, A.M. Macdonald, P.S.K. Liu, W.R. Leaitch, K.G. Anlauf, L. Jaegle, T.D. Fairlie, and D.L. Westphal, 2007: Trans-Pacific transport of Saharan dust to western North America: A case study. Journal of Geophysical Research - Atmospheres, 112, D01103.

McVicar, T.R., T.G. Van Niel, L.T. Li, M.L. Roderick, D.P. Rayner, L. Ricciardulli, and R.J. Donohue, 2008: Wind speed climatology and trends for Australia, 1975-2006: Capturing the stilling phenomenon and comparison with near-surface reanalysis output. Geophysical Research Letters, 35, L20403.

Mearns, L.O., W. Gutowski, R. Jones, R. Leung, S. McGinnis, A. Nunes, and Y. Qian, 2009: A regional climate change assessment program for North America. Eos Transactions (AGU), 90(36), 311.

Meehl, G.A. and C. Tebaldi, 2004: More intense, more frequent, and longer lasting heat waves in the 21st century. Science, 305(5686 ), 994-997.

Meehl, G.A., J.M. Arblaster, and C. Tebaldi, 2005: Understanding future patterns of increased precipitation intensity in climate model simulations. Geophysical Research Letters, 32, L18719.

Meehl, G.A., C. Covey, T. Delworth, M. Latif, B. McAvaney, J.F.B. Mitchell, R.J. Stouffer, and K.E. Taylor, 2007a: The WCRP CMIP3 multimodel dataset - A new era in climate change research. Bulletin of the American Meteorological Society, 88(9), 1383-1394.

Meehl, G.A., T.F. Stocker, W.D. Collins, P. Friedlingstein, A.T. Gaye, J.M. Gregory, A. Kitoh, R. Knutti, J.M. Murphy, A. Noda, S.C.B. Raper, I.G. Watterson, A.J. Weaver, and Z.C. Zhao, 2007b: Global climate projections. In: Climate Change 2007: The Physical Science Basis. Contribution of Working Group I to the Fourth Assessment Report of the Intergovernmental Panel on Climate Change [Solomon, S., D. Qin, M. Manning, Z. Chen, M. Marquis, K.B. Averyt, M. Tignor and H.L. Miller (eds.)]. Cambridge University Press, Cambridge, UK, and New York, NY, pp. 747-845.

Meehl, G.A., J.M. Arblaster, and W.D. Collins, 2008: Effects of black carbon aerosols on the Indian monsoon. Journal of Climate, 21(12), 2869-2882.

Meehl, G.A., J.M. Arblaster, K. Matthes, F. Sassi, and H. van Loon, 2009a: Amplifying the Pacific climate system response to a small 11-year solar cycle forcing. Science, 325(5944), 1114-1118.

Meehl, G.A., A. Hu, and B.D. Santer, 2009b: The mid-1970s climate shift in the Pacific and the relative roles of forced versus inherent decadal variability. Journal of Climate, 22(3), 780-792.

Meehl, G.A., C. Tebaldi, G. Walton, D. Easterling, and L. McDaniel, 2009c: The relative increase of record high maximum temperatures compared to record low minimum temperatures in the U.S. Geophysical Research Letters, 36, L23701. 
Meinshausen, M., N. Meinshausen, W. Hare, S.C.B. Raper, K. Frieler, R. Knutti, D.J. Frame, and M.R. Allen, 2009: Greenhouse-gas emission targets for limiting global warming to $2^{\circ} \mathrm{C}$. Nature, $\mathbf{4 5 8 ( 7 2 4 2 ) ,}$, 1158-1162.

Menendez, C.G., M. de Castro, J.P. Boulanger, A. D'Onofrio, E. Sanchez, A.A. Sorensson, J. Blazquez, A. Elizalde, D. Jacob, H. Le Treut, Z.X. Li, M.N. Nunez, N. Pessacg, S. Pfeiffer, M. Rojas, A. Rolla, P. Samuelsson, S.A. Solman, and C. Teichmann, 2010: Downscaling extreme month-long anomalies in southern South America. Climatic Change, 98(3-4), 379-403.

Menendez, M. and P.L. Woodworth, 2010: Changes in extreme high water levels based on a quasi-global tide-gauge dataset. Journal of Geophysical Research, 115, C10011.

Menendez, M., F.J. Mendez, I.J. Losada, and N.E. Graham, 2008: Variability of extreme wave heights in the northeast Pacific Ocean based on buoy measurements. Geophysical Research Letters, 35, L22607.

Menne, M.J., and C.N. Williams Jr., 2009: Homogenization of temperature series via pairwise comparisons. Journal of Climate, 22(7), 1700-1717.

Merrifield, M.A., Y.L. Firing, and J.J. Marra, 2007: Annual climatologies of extreme water levels. In: Aha Hulikoa: Extreme Events. Proceedings of the Hawaiian Winter Workshop, University of Hawaii at Manoa, 23 -26 Jan 2007. SOEST, University of Hawaii, Manoa, HI, pp. 27-32.

Miller, R., G. Schmidt, and D. Shindell, 2006: Forced annular variations in the 20th century Intergovernmental Panel on Climate Change Fourth Assessment Report models. Journal of Geophysical Research, 111, D18101.

Mills, G.A., 2005: A re-examination of the synoptic and mesoscale meteorology of Ash Wednesday 1983. Australian Meteorological Magazine, 54(1), 35-55.

Milly, P.C.D. and K.A. Dunne, 2011: Hydrologic adjustment of climate-model projections: The potential pitfall of potential evapotranspiration. Earth Interactions, 15(1), 1-14.

Milly, P.C.D., R.T. Wetherald, K.A. Dunne, and T.L. Delworth, 2002: Increasing risk of great floods in a changing climate. Nature, 415(6871), 514-517.

Mimura, N., 1999: Vulnerability of island countries in the South Pacific to sea level rise and climate change. Climate Research, 12(2-3), 137-143.

Min, S.-K., X. Zhang, and F. Zwiers, 2008: Human-induced arctic moistening. Science, 320(5875), 518-520.

Min, S.-K., X. Zhang, F.W. Zwiers, and G.C. Hegerl, 2011: Human contribution to more intense precipitation extremes. Nature, 470(7334), 378-381.

Min, S.K., and A. Hense, 2007: A Bayesian assessment of climate change using multimodel ensembles. Part II: Regional and seasonal mean surface temperatures. Journal of Climate, 20(12), 2769-2790.

Mitrovica, J.X., M.E. Tamisiea, E.R. Ivins, L.L.A. Vermeersen, G.A. Milne, and K. Lambeck, 2010: Surface mass loading on a dynamic earth: complexity and contamination in the geodetic analysis of global sea-level trends. In: Understanding Sea-Level Rise and Variability [Church, J.A., P.L. Woodworth, T. Aarup, and W.S. Wilson (eds.)]. Wiley-Blackwell, Chichester, UK, pp. 285-325.

Moberg, A., P.D. Jones, D. Lister, A. Walther, M. Brunet, J. Jacobeit, L.V. Alexander, P.M. Della-Marta, J. Luterbacher, P. Yiou, D. Chen, A.M.G.K. Tank, O. Saladie, J. Sigro, E. Aguilar, H. Alexandersson, C. Almarza, I. Auer, M. Barriendos, M. Begert, H. Bergstroem, R. Boehm, C.J. Butler, J. Caesar, A. Drebs, D. Founda, F.-W. Gerstengarbe, G. Micela, M. Maugeri, H. Osterle, K. Pandzic, M. Petrakis, L. Srnec, R. Tolasz, H. Tuomenvirta, P.C. Werner, H. Linderholm, A. Philipp, H. Wanner, and E. Xoplaki, 2006: Indices for daily temperature and precipitation extremes in Europe analyzed for the period 1901-2000. Journal of Geophysical Research - Atmospheres, 111, D22106.

Moise, A.F., and R. Colman, 2009: Tropical Australia and the Australian Monsoon: general assessment and projected changes. In: 18th World IMACS Congress and MODSIM09 International Congress on Modelling and Simulation. Modelling and Simulation Society of Australia and New Zealand and International Association for Mathematics and Computers in Simulation, July 2009 [Anderssen, R.S., R.D. Braddock, and L.T.H. Newham (eds.)]. pp. 2042-2048.

Morgenstern, O., H. Akiyoshi, S. Bekki, P. Braesicke, N. Butchart, M.P. Chipperfield, D. Cugnet, M. Deushi, S.S. Dhomse, R.R. Garcia, A. Gettelman, N.P. Gillett, S.C. Hardiman, J. Jumelet, D.E. Kinnison, J.F. Lamarque, F. Lott, M. Marchand, M. Michou, T. Nakamura, D. Olivie, T. Peter, D. Plummer, J.A. Pyle, E. Rozanov, D. Saint-Martin, J.F. Scinocca, K. Shibata, M. Sigmond, D. Smale, H. Teyssedre, W. Tian, A. Voldoire, and Y. Yamashita, 2010: Anthropogenic forcing of the Northern Annular Mode in CCMVal-2 models. Journal of Geophysical Research -Atmospheres, 115, D00M03.
Mori, N., T. Yasuda, H. Mase, T. Tom, and Y. Oku, 2010: Projection of extreme wave climate change under global warming. Hydrological Research Letters, 4, 15-19.

Mudelsee, M., M. Borngen, G. Tetzlaff, and U. Grunewald, 2003: No upward trends in the occurrence of extreme floods in central Europe. Nature, 425(6954), 166-169.

Muir-Wood, R., 2000: Deglaciation Seismotectonics: a principal influence on intraplate seismogenesis at high latitudes. Quaternary Science Reviews, 19(14-15), 1399-1411.

Mullan, B., D. Wratt, S. Dean, M. Hollis, S. Allan, T. Williams, and G. Kenny, 2008: Climate Change Effects and Impacts Assessment: A Guidance Manual for Local Government in New Zealand. 2nd ed. Ministry for the Environment, Wellington, New Zealand, $149 \mathrm{pp}$.

Müller, W.A. and E. Roeckner, 2008: ENSO teleconnections in projections of future climate in ECHAM5/MPI-OM. Climate Dynamics, 31(5), 533-549.

Murakami, H., B. Wang, and A. Kitoh, 2011: Future change of western North Pacific typhoons: Projections by a 20-km-mesh global atmospheric model. Journal of Climate, 24(4), 1154-1169.

Murphy, J.M., B.B.B. Booth, M. Collins, G.R. Harris, D.M.H. Sexton, and M.J. Webb, 2007: A methodology for probabilistic predictions of regional climate change from perturbed physics ensembles. Philosophical Transactions of the Royal Society A, 365(1857), 1993-2028.

Murphy, J.M., D.M. Sexton, G.J. Jenkins, B.B.B. Booth, C.C. Brown, R.T. Clark, M. Collins, G.R. Harris, E.J. Kendon, R.A. Betts, S.J. Brown, K.A. Humphrey, M.P. McCarthy, R.E. McDonald, A. Stephens, C. Wallace, R. Warren, R. Wilby, and R.A. Wood, 2009: UK Climate Projections Science Report: Climate change projections. Met Office Hadley Centre, Exeter, UK.

Nakaegawa, T. and W. Vergara, 2010: First projection of climatological mean river discharges in the Magdalena River Basin, Colombia, in a changing climate during the 21st century. Hydrological Research Letters, 4, 50-54.

Nakamura, M., S. Kanada, Y. Wakazuki, C. Muroi, A. Hashimoto, T. Kato, A. Noda, M. Yoshizaki, and K. Yasunaga, 2008: Effects of global warming on heavy rainfall during the Baiu season projected by a cloud-system-resolving model. Journal of Disaster Research, 3(1), 15-24.

Nandintsetseg, B., J.S. Greene, and C.E. Goulden, 2007: Trends in extreme daily precipitation and temperature near lake Hövsgöl, Mongolia. International Journal of Climatology, 27(3), 341-347.

Narama, C., Y. Shimamura, D. Nakayama, and K. Abdrakhmatov, 2006: Recent changes of glacier coverage in the western Terskey-Alatoo range, Kyrgyz Republic, using Corona and Landsat. Annals of Glaciology, 43(1), 223-229.

National Climate Centre, 2009: The Exceptional January-February 2009 Heatwave in Southeastern Australia. Special Climate Statement 17 (www.bom.gov.au/ climate/current/statements/scs17d.pdf), Bureau of Meteorology, Melbourne, Australia.

Neill, S.P., J.D. Scourse, G.R. Bigg, and K. Uehara, 2009: Changes in wave climate over the northwest European shelf seas during the last 12,000 years. Journal of Geophysical Research - Oceans, 114, C06015.

Neu, U., 2008: Is recent major hurricane activity normal? Nature, 451(7181), E5.

New, M., B. Hewitson, D.B. Stephenson, A. Tsiga, A. Kruger, A. Manhique, B. Gomez, C.A.S. Coelho, D.N. Masisi, E. Kulunaga, E. Mbambalala, F. Adesina, H. Saleh, J. Kanyanga, J. Adosi, L. Bulane, L. Fortunata, M.L. Mdoka, and R. Lajoie, 2006: Evidence of trends in daily climate extremes over southern and west Africa. Journal of Geophysical Research, 111, D14102.

Newhall, C.G. and R.S. Punongbayan (eds.), 1996: Fire and Mud: Eruptions and Lahars of Mount Pinatubo, Philippines. University of Washington Press, Seattle, WA, $1126 \mathrm{pp}$.

Niall, S. and K. Walsh, 2005: The impact of climate change on hailstorms in southeastern Australia. International Journal of Climatology, 25(14), 1933-1952.

Nicholls, N., 1995: Long-term climate monitoring and extreme events. Climatic Change, 31(2-4), 231-245.

Nicholls, N., 2008: Recent trends in the seasonal and temporal behaviour of the El Niño Southern Oscillation. Geophysical Research Letters, 35, L19703.

Nicholls, N., 2010: Local and remote causes of the southern Australian autumnwinter rainfall decline, 1958-2007. Climate Dynamics, 34(6), 835-845. 
Nicholls, N. and L. Alexander, 2007: Has the climate become more variable or extreme? Progress 1992-2006. Progress in Physical Geography, 31(1), 77-87.

Nicholls, R.J., 2010: Impacts of and responses to sea-level rise. In: Understanding Sea-Level Rise and Variability [Church, J.A., P.L. Woodworth, T. Aarup, and W.S. Wilson (eds.)]. Wiley-Blackwell, Chichester, UK, pp. 17-51.

Nicholls, R.J. and A.C. de la Vega-Leinert, 2008: Implications of sea-level rise for Europe's coasts: An introduction. Journal of Coastal Research, 24(2), 285-287.

Nicholls, R.J., P.P. Wong, V.R. Burkett, J.O. Codignotto, J.E. Hay, R.F. McLean, S. Ragoonaden, and C.D. Woodroffe, 2007: Coastal systems and low-lying areas. In: Climate Change 2007. Impacts, Adaptation and Vulnerability. Contribution of Working Group II to the Fourth Assessment Report of the Intergovernmental Panel on Climate Change [Parry, M.L., O.F. Canziani, J.P. Palutikof, P.J. Van Der Linden, and C.E. Hanson (eds.)]. Cambridge University Press, Cambridge, UK, pp. 315-356.

Nieto-Ferreira, R. and T. Rickenbach, 2011: Regionality of monsoon onset in South America: a three stage conceptual model. International Journal of Climatology, 31(9), 1309-1321.

Nissen, K.M., G.C. Leckebusch, J.G. Pinto, D. Renggli, S. Ulbrich, and U. Ulbrich, 2010: Cyclones causing wind storms in the Mediterranean: characteristics, trends and links to large-scale patterns. Natural Hazards and Earth System Sciences, 10(7), 1379-1391.

Niu, F., G. Cheng, W. Ni, and D. Jin, 2005: Engineering-related slope failure in permafrost regions of the Qinghai-Tibet Plateau. Cold Regions Science and Technology, 42(3), 215-225.

Noetzli, J., S. Gruber, T. Kohl, N. Salzmann, and W. Haeberli, 2007: Three-dimensional distribution and evolution of permafrost temperatures in idealized highmountain topography. Journal of Geophysical Research - Earth Surface, 112, F02S13.

Noetzli, J. and S. Gruber, 2009: Transient thermal effects in Alpine permafrost. The Cryosphere, 3(1), 85-99.

Nott, J., J. Haig, H. Neil, and D. Gillieson, 2007: Greater frequency variability of landfalling tropical cyclones at centennial compared to seasonal and decadal scales. Earth and Planetary Science Letters, 255(3-4), 367-372.

Nott, J., S. Smithers, K. Walsh, and E. Rhodes, 2009: Sand beach ridges record 6000 year history of extreme tropical cyclone activity in northeastern Australia. Quaternary Science Reviews, 28(15-16), 1511-1520.

Nunez, M.N., S.A. Solman, and M.F. Cabre, 2009: Regional climate change experiments over southern South America. II: Climate change scenarios in the late twentyfirst century. Climate Dynamics, 32(7-8), 1081-1095.

Nyberg, J., B.A. Malmgren, A. Winter, M.R. Jury, K. Halimeda Kilbourne, and T.M. Quinn, 2007: Low Atlantic hurricane activity in the 1970s and 1980s compared to the past 270 years. Nature, $\mathbf{4 4 7 ( 7 1 4 5 ) , 6 9 8 - 7 0 1 . ~}$

0'Gorman, P.A., 2010: Understanding the varied response of the extratropical storm tracks to climate change. Proceedings of the National Academy of Sciences, 107(45), 19176-19180.

0'Gorman, P.A. and T. Schneider, 2008: Energy of midlatitude transient eddies in idealized simulations of changed climates. Journal of Climate, 21(22), 5797-5806.

0'Gorman, P.A. and T. Schneider, 2009a: The physical basis for increases in precipitation extremes in simulations of 21 st-century climate change. Proceedings of the National Academy of Sciences, 106(35), 14773-14777.

O'Gorman, P.A., and T. Schneider, 2009b: Scaling of precipitation extremes over a wide range of climates simulated with an idealized GCM. Journal of Climate, 22(21), 5676-5685.

Oki, T., T. Nishimura, and P. Dirmeyer, 1999: Assessment of annual runoff from land surface models using Total Runoff Integrating Pathways (TRIP). Journal of the Meteorological Society of Japan, 77(1), 235-255

Okin, G.S., D.A. Gillette, and J.E. Herrick, 2006: Multi-scale controls on and consequences of aeolian processes in landscape change in arid and semi-arid environments. Journal of Arid Environments, 65(2), 253-275.

Oouchi, K., J. Yoshimura, H. Yoshimura, R. Mizuta, S. Kusunoki, and A. Noda, 2006: Tropical cyclone climatology in a global-warming climate as simulated in a 20 km-mesh global atmospheric model: Frequency and wind intensity analyses. Journal of the Meteorological Society of Japan, 84(2), 259-276.
Oppikofer, T., M. Jaboyedoff, and H.R. Keusen, 2008: Collapse at the eastern Eiger flank in the Swiss Alps. Nature Geoscience, 1(8), 531-535.

Orlowsky, B. and S.I. Seneviratne, 2011: Global changes in extremes events: Regional and seasonal dimension. Climatic Change, doi:10.1007/s10584-011-0122-9.

Orsolini, Y. and A. Sorteberg, 2009: Projected changes in Eurasian and Arctic Summer cyclones under global warming in the Bergen Climate Model. Atmospheric and Oceanic Science Letters, 2(1), 62.

Osterkamp, T.E., 2005: The recent warming of permafrost in Alaska. Global and Planetary Change, 49, 187-202.

Osterkamp, T.E., 2007: Characteristics of the recent warming of permafrost in Alaska. Journal of Geophysical Research, 112, F02S02.

Osterkamp, T.E., M.T. Jorgenson, E.A.G. Schuur, Y.L. Shur, M.Z. Kanevskiy, J.G. Vogel, and V.E. Tumskoy, 2009: Physical and ecological changes associated with warming permafrost and thermokarst in Interior Alaska. Permafrost and Periglacial Processes, 20(3), 235-256.

Osti, R., and S. Egashira, 2009: Hydrodynamic characteristics of the Tam Pokhari glacial lake outburst flood in the Mt. Everest region, Nepal. Hydrological Processes, 23(20), 2943-2955.

Otto-Bliesner, B.L., R. Schneider, E.C. Brady, M. Kucera, A. Abe-Ouchi, E. Bard, P. Braconnot, M. Crucifix, C.D. Hewitt, M. Kageyama, O. Marti, A. Paul, A. RosellMelé, C. Waelbroeck, S.L. Weber, M. Weinelt, and Y. Yu, 2009: A comparison of PMIP2 model simulations and the MARGO proxy reconstruction for tropical sea surface temperatures at last glacial maximum. Climate Dynamics, 32(6), 799-815.

Paciorek, C.J., J.S. Risbey, V. Ventura, and R.D. Rosen, 2002: Multiple indices of Northern Hemisphere cyclone activity, winters 1949-99. Journal of Climate, 15(13), 1573-1590.

Paeth, H. and H.-P. Thamm, 2007: Regional modelling of future African climate north of 15 degrees $S$ including greenhouse warming and land degradation. Climatic Change, 83(3), 401-427.

Pagli, C. and F. Sigmundsson, 2008: Will present day glacier retreat increase volcanic activity? Stress induced by recent glacier retreat and its effect on magmatism at the Vatnajökull ice cap, Iceland. Geophysical Research Letters, 35, L09304.

Pall, P., M.R. Allen, and D.A. Stone, 2007: Testing the Clausius-Clapeyron constraint on changes in extreme precipitation under $\mathrm{CO}_{2}$ warming. Climate Dynamics, 28(4), 351-363

Pall, P., T. Aina, D.A. Stone, P.A. Stott, T. Nozawa, A.G.J. Hilbberts, D. Lohmann, and M.R. Allen, 2011: Anthropogenic greenhouse gas contribution to flood risk in England and Wales in autumn 2000. Nature, 470(7334), 382-385.

Palmer, W.C., 1965: Meteorological Drought. Report 45, US Weather Bureau, Washington, DC.

Pan, Z.T., R.W. Arritt, E.S. Takle, W.J. Gutowski, C.J. Anderson, and M. Segal, 2004: Altered hydrologic feedback in a warming climate introduces a "warming hole." Geophysical Research Letters, 31, L17109.

Pattanaik, D.R. and M. Rajeevan, 2010: Variability of extreme rainfall events over India during southwest monsoon season. Meteorological Applications, 17(1), 88-104.

Paul, F. and W. Haeberli, 2008: Spatial variability of glacier elevation changes in the Swiss Alps obtained from two digital elevation models. Geophysical Research Letters, 35, L21502.

Paul, F., A. Kääb, M. Maisch, T. Kellenberger, and W. Haeberli, 2004: Rapid disintegration of Alpine glaciers observed with satellite data. Geophysical Research Letters, 31, L21402.

Pavan, V., R. Tomozeiu, C. Cacciamani, and M. Di Lorenzo, 2008: Daily precipitation observations over Emilia-Romagna: mean values and extremes. International Journal of Climatology, 28(15), 2065-2079.

Peel, M.C. and T.A. McMahon, 2006: Continental runoff: A quality-controlled global runoff data set. Nature, 444(E14-E15)

Penalba, O.C. and F.A. Robledo, 2010: Spatial and temporal variability of the frequency of extreme daily rainfall regime in the La Plata Basin during the 20th century. Climate Change, 98(3-4), 531-550.

Perkins, S.E., A.J. Pitman, and S.A. Sisson, 2009: Smaller projected increases in 20year temperature returns over Australia in skill-selected climate models. Geophysical Research Letters, 36, L06710. 
Peters, E., P.J.J.F. Torfs, H.A.J. van Lanen, and G. Bier, 2003: Propagation of drought through groundwater - a new approach using linear reservoir theory. Hydrological Processes, 17(15), 3023-3040.

Peterson, T.C. and M.J. Manton, 2008: Monitoring changes in climate extremes - A tale of international collaboration. Bulletin of the American Meteorological Society, 89(9), 1266-1271.

Peterson, T.C., M.A. Taylor, R. Demerritte, D.L. Duncombe, S. Burton, F. Thompson, A. Porter, M. Mercedes, E. Villegas, R.S. Fils, A. Klein Tank, A. Martis, R. Warner, A. Joyette, W. Mills, L. Alexander, and B. Gleason, 2002: Recent changes in climate extremes in the Caribbean region. Journal of Geophysical Research Atmospheres, 107, 4601.

Peterson, T.C., X. Zhang, M. Brunet-India, and J.L. Vazquez-Aguirre, 2008a: Changes in North American extremes derived from daily weather data. Journal of Geophysical Research - Atmospheres, 113, D07113.

Peterson, T.C., D. Anderson, S.J. Cohen, M. Cortez, R. Murname, C. Parmesan, D. Phillips, R. Pulwarty, and J. Stone, 2008b: Why weather and climate extremes matter. In: Weather and Climate Extremes in a Changing Climate. Regions of Focus: North America, Hawaii, Caribbean, and U.S. Pacific Islands. [Karl, T.R., G.A. Meehl, D.M. Christopher, S.J. Hassol, A.M. Waple, and W.L. Murray (eds.)]. A Report by the U.S. Climate Change Science Program and the Subcommittee on Global Change Research, Washington, DC, pp. 11-33.

Petoukhov, V. and V.A. Semenov, 2010: A link between reduced Barents-Kara sea ice and cold winter extremes over northern continents. Journal of Geophysical Research-Atmospheres, 115, D21111.

Petrow, T. and B. Merz, 2009: Trends in flood magnitude, frequency and seasonality in Germany in the period 1951-2002. Journal of Hydrology, 371(1-4), 129-141.

Pezza, A.B., and I. Simmonds, 2008: Large-scale factors in tropical and extratropical cyclone transition and extreme weather events. Trends and Directions in Climate Research: Annals of the New York Academy of Sciences, 1146, 189-211.

Pezza, A.B., I. Simmonds, and J.A. Renwick, 2007: Southern Hemisphere cyclones and anticyclones: Recent trends and links with decadal variability in the Pacific Ocean. International Journal of Climatology, 27(11), 1403-1419.

Pfeffer, W.T., J.T. Harper, and S. O'Neel, 2008: Kinematic constraints on glacier contributions to 21st-century sea-level rise. Science, 321(5894), 1340-1343.

Philander, S.G., 1990: El Niño, La Niña and the Southern Oscillation. Academic Press, San Diego, CA, 293 pp.

Piani, C., J.O. Haerter, and E. Coppola, 2010: Statistical bias correction for daily precipitation in regional climate models over Europe. Theoretical and Applied Climatology, 99(1-2), 187-192.

Piao, S., P. Friedlingstein, P. Ciais, N. de Noblet-Ducoudré, D. Labat, and S. Zaehle, 2007: Changes in climate and land use have a larger direct impact than rising $\mathrm{CO}_{2}$ on global river runoff trends. Proceedings of the National Academy of Sciences, 104(39), 15242-15247.

Pielke Jr., R.A., J. Gratz, C.W. Landsea, D. Collins, M. Saunders, and R. Musulin, 2008: Normalized hurricane damages in the United States. Natural Hazards Review, 9(1), 29-42.

Pinto, J.G., T. Spangehl, U. Ulbrich, and P. Speth, 2006: Assessment of winter cyclone activity in a transient ECHAM4-OPYC3 GHG experiment. Meteorologische Zeitschrift, 15(3), 279-291.

Pinto, J.G., U. Ulbrich, G.C. Leckebusch, T. Spangehl, M. Reyers, and S. Zacharias, 2007a: Changes in storm track and cyclone activity in three SRES ensemble experiments with the ECHAM5/MPI-OM1 GCM. Climate Dynamics, 29(2-3), 195-210.

Pinto, J.G., E.L. Frohlich, G.C. Leckebusch, and U. Ulbrich, 2007b: Changing European storm loss potentials under modified climate conditions according to ensemble simulations of the ECHAM5/MPI-OM1 GCM. Natural Hazards and Earth System Sciences, 7(1), 165-175.

Pinto, J.G., S. Zacharias, A.H. Fink, G.C. Leckebusch, and U. Ulbrich, 2009: Factors contributing to the development of extreme North Atlantic cyclones and their relationship with the NAO. Climate Dynamics, 32(5), 711-737.

Pinto, J.G., M. Reyers, and U. Ulbrich, 2011: The variable link between PNA and NAO in observations and in multi-century CGCM simulations. Climate Dynamics, 36(1), 337-354
Pirazzoli, P.A., and A. Tomasin, 2003: Recent near-surface wind changes in the central Mediterranean and Adriatic areas. International Journal of Climatology, 23(8), 963-973.

Pitman, A.J., N. de Noblet-Ducoudré, F.T. Cruz, E. Davin, G.B. Bonan, V. Brovkin, C. M., D. C., V. Gayler, B.J.J.M. van den Hurk, P.J. Lawrence, M.K. van der Molen, C. Müller, C.H. Reick, S.I. Seneviratne, B.J. Strengers, and A. Voldoire, 2009: Uncertainties in climate responses to past land cover change: first results from the LUCID intercomparison study. Geophysical Research Letters, 36, L14814.

Planton, S., M. Deque, F. Chauvin, and L. Terray, 2008: Expected impacts of climate change on extreme climate events. Comptes Rendus Geoscience, 340(9-10), 564-574.

Portmann, R.W., S. Solomon, and G.C. Hegerl, 2009: Linkages between climate change, extreme temperature and precipitation across the United States. Proceedings of the National Academy of Sciences, 106(18), 7324-7329.

Poulter, B., F. Hattermann, E.D. Hawkins, S. Zaehle, S. Sitch, N. Restrepo-Coupe, U. Heyder, and W. Cramer, 2010: Robust dynamics of Amazon dieback to climate change with perturbed ecosystem model parameters. Global Change Biology, 16(9), 2476-2495

Power, S.B. and I.N. Smith, 2007: Weakening of the Walker Circulation and apparent dominance of El Niño both reach record levels, but has ENSO really changed? Geophysical Research Letters, 34, L18702.

Prospero, J.M. and P.J. Lamb, 2003: African droughts and dust transport to the Caribbean: Climate change implications. Science, 302(5647), 1024-1027.

Prospero, J.M., E. Blades, R. Naidu, and M.C. Lavoie, 2009: Reply to: African dust and asthma in the Caribbean-medical and statistical perspectives by M.A. Monteil and R. Antoine. International Journal of Biometeorology, 53(5), 383-385.

Prudhomme, C. and H. Davies, 2009: Assessing uncertainties in climate change impact analyses on the river flow regimes in the UK. Part 2: future climate. Climatic Change, 93(1-2), 197-222.

Pruszak, Z., and E. Zawadzka, 2008: Potential implications of sea-level rise for Poland. Journal of Coastal Research, 24(2), 410-422.

Pryor, S.C., R.J. Barthelmie, and E.S. Riley, 2007: Historical evolution of wind climates in the USA. Journal of Physics: Conference Series, 75, 012065.

Pryor, S.C., J.A. Howe, and K.E. Kunkel, 2009: How spatially coherent and statistically robust are temporal changes in extreme precipitation in the contiguous USA? International Journal of Climatology, 29(1), 31-45.

Purvis, M.J., P.D. Bates, and C.M. Hayes, 2008: A probabilistic methodology to estimate future coastal flood risk due to sea level rise. Coastal Engineering, 55(12), 1062-1073.

Qian, B., S. Gameda, and H. Hayhoe, 2008: Performance of stochastic weather generators LARS-WG and AAFC-WG for reproducing daily extremes of diverse Canadian climates. Climate Research, 37(1), 17-33.

Raff, D.A., T. Pruitt, and L.D. Brekke, 2009: A framework for assessing flood frequency based on climate projection information. Hydrology and Earth System Sciences, 13(11), 2119-2136.

Rahimzadeh, F., A. Asgari, and E. Fattahi, 2009: Variability of extreme temperature and precipitation in Iran during recent decades. International Journal of Climatology, 29(3), 329-343.

Rahmstorf, S., 2007: A semi-empirical approach to projecting future sea-level rise. Science, 315(5810), 368-370.

Rahmstorf, S., M. Crucifix, A. Ganopolski, H. Goosse, I. Kamenkovich, R. Knutti, G. Lohmann, R. Marsh, L.A. Mysak, Z.M. Wang, and A.J. Weaver, 2005: Thermohaline circulation hysteresis: A model intercomparison. Geophysical Research Letters, 32, L23605.

Raia, A., and I.F.D. Cavalcanti, 2008: The life cycle of the South American monsoon system. Journal of Climate, 21(23), 6227-6246.

Raible, C.C., 2007: On the relation between extremes of midlatitude cyclones and the atmospheric circulation using ERA40. Geophysical Research Letters, 34, L07703.

Raible, C.C., P.M. Della-Marta, C. Schwierz, H. Wernli, and R. Blender, 2008: Northern hemisphere extratropical cyclones: A comparison of detection and tracking methods and different reanalyses. Monthly Weather Review, 136(3), 880-897. 
Räisänen, J., 2007: How reliable are climate models? Tellus Series $A$ - Dynamic Meteorology and Oceanography, 59, 2-29.

Raje, D., and P.P. Mujumdar, 2010: Constraining uncertainty in regional hydrologic impacts of climate change: Nonstationarity in downscaling. Water Resources Research, 46, W07543.

Rajeevan, M., J. Bhate, and A.K. Jaswal, 2008: Analysis of variability and trends of extreme rainfall events over India using 104 years of gridded daily rainfall data. Geophysical Research Letters, 35, L18707.

Rajendran, K., and A. Kitoh, 2008: Indian summer monsoon in future climate projection by a super high-resolution global model. Current Science, 95(11), 1560-1569.

Ramsay, H.A., and A.H. Sobel, 2011: The effects of relative and absolute sea surface temperature on tropical cyclone potential intensity using a single column model. Journal of Climate, 24(1), 183-193.

Ranasinghe, R., and M. Stive, 2009: Rising seas and retreating coastlines. Climatic Change, 97(3), 465-468.

Ranasinghe, R., R. McLoughlin, A. Short, and G. Symonds, 2004: The Southern Oscillation Index, wave climate, and beach rotation. Marine Geology, 204(3-4), 273-287.

Randall, D.A., R.A. Wood, S. Bony, R. Colman, T. Fichefet, J. Fyfe, V. Kattsov, A. Pitman, J. Shukla, J. Srinivasan, R.J. Stouffer, A. Sumi, and K.E. Taylor, 2007: Climate models and their evaluation. In: Climate Change 2007: The Physical Science Basis. Contribution of Working Group I to the Fourth Assessment Report of the Intergovernmental Panel on Climate Change [Solomon, S., D. Qin, M. Manning, Z. Chen, M. Marquis, K.B. Averyt, M. Tignor and H.L. Miller (eds.)]. Cambridge University Press, Cambridge, UK, and New York, NY, pp. 589-662.

Rappaport, E.N., 2000: Loss of life in the United States associated with recent Atlantic tropical cyclones. Bulletin of the American Meteorological Society, 81(9), 2065-2073.

Rauthe, M., M. Kunz, and C. Kottmeier, 2010: Changes in wind gust extremes over Central Europe derived from a small ensemble of high resolution regional climate models. Meteorologische Zeitschrift, 19(3), 299-312.

Ravanel, L. and P. Deline, 2011: Climate influence on rockfalls in high-Alpine steep rockwalls: The north side of the Aiguilles de Chamonix (Mont Blanc massif) since the end of the 'Little Ice Age'. The Holocene, 21(2), 357-365.

Re, M., and V. Ricardo Barros, 2009: Extreme rainfalls in SE South America. Climatic Change, 96(1-2), 119-136.

Rebetez, M., O. Dupont, and M. Giroud, 2008: An analysis of the July 2006 heatwave extent in Europe compared to the record year of 2003. Theoretical and Applied Climatology, 95(1-2), 1-7.

Redmond, K.T., Y. Enzel, P.K. House, and F. Biondi, 2002: Climate impact on flood frequency at the decadal to millennial time scales. In: Ancient Floods, Modern Hazards: Principles and Applications of Paleoflood Hydrology [House, P.K., R.H. Webb, V.R. Baker, and D.R. Levish (eds.)]. Vol. 5. American Geophysical Union Water Science and Applications, AGU, Washington, DC, pp. 21-46.

Regonda, S.K., B. Rajagopalan, M. Clark, and J. Pitlick, 2005: Seasonal cycle shifts in hydroclimatology over the western United States. Journal of Climate, 18(2), 372-384.

Rehman, S., 2010: Temperature and rainfall variation over Dhahran, Saudi Arabia, (1970-2006). International Journal of Climatology, 30(3), 445-449.

Reichert, B.K., L. Bengtsson, and J. Oerlemans, 2002: Recent glacier retreat exceeds internal variability. Journal of Climate, 15(21), 3069-3081.

Reichstein, M., P. Ciais, D. Papale, R. Valentini, S. Running, N. Viovy, W. Cramer, A. Granier, J. Ogée, V. Allard, M. Aubinet, C. Bernhofer, N. Buchmann, A. Carrara, T. Grünwald, M. Heimann, B. Heinesch, A. Knohl, W. Kutsch, D. Loustau, G. Manca, G. Matteucci, F. Miglietta, J.M. Ourcival, K. Pilegaard, J. Pumpanen, S. Rambal, S. Schaphoff, S. Seufert, J.F. Soussana, M.J. Sanz, T. Vesala, and M. Zhao, 2007: Reduction of ecosystem productivity and respiration during the European summer 2003 climate anomaly: a joint flux tower, remote sensing and modeling analysis. Global Change Biology, 13(3), 634-651.

Rein, B., A. Lückge, L. Reinhardt, F. Sirocko, A. Wolf, and W.C. Dullo, 2005: El Niño variability off Peru during the last 20,000 years. Paleoceanography, 20, PA4003.
Renard, B., and M. Lang, 2007: Use of a Gaussian copula for multivariate extreme value analysis: Some case studies in hydrology. Advances in Water Resources, 30(4), 897-912.

Renard, B., M. Lang, P. Bois, A. Dupeyrat, O. Mestre, H. Niel, E. Sauquet, C. Prudhomme, S. Parey, E. Paquet, L. Neppel, and J. Gailhard, 2008: Regional methods for trend detection: Assessing field significance and regional consistency. Water Resources Research, 44, W08419.

Reynolds, J.M., 1998: High-altitude glacial lake hazard assessment and mitigation: a Himalayan perspective. Geological Society, London, Engineering Geology Special Publications, 15(1), 25-34.

Reynolds, J.M., A. Dolecki, and C. Portocarrero, 1998: The construction of a drainage tunnel as part of glacial lake hazard mitigation at Hualcan, Cordillera Blanca, Peru. Engineering Geology Special Publications, 15(1), 41-48.

Richardson, S.D. and J.M. Reynolds, 2000: An overview of glacial hazards in the Himalayas. Quaternary International, 65, 31-47.

Rickenmann, D. and M. Zimmermann, 1993: The 1987 debris flows in Switzerland: documentation and analysis. Geomorphology (Amsterdam), 8(2-3), 175-189.

Rignot, E., I. Velicogna, M.R. van den Broeke, A. Monaghan, and J. Lenaerts, 2011: Acceleration of the contribution of the Greenland and Antarctic ice sheets to sea level rise. Geophysical Research Letters, 38(L05503).

Rist, A., and M. Phillips, 2005: First results of investigations on hydrothermal processes within the active layer above alpine permafrost in steep terrain. Norsk Geografisk Tidsskrift, 59(2), 177-183.

Robeson, S., 2004: Trends in time-varying percentiles of daily minimum and maximum temperature over North America. Geophysical Research Letters, 31, L04203.

Robinson, W.A., 2000: A baroclinic mechanism for the eddy feedback on the zonal index. Journal of the Atmospheric Sciences, 57(3), 415-422.

Robock, A., K.Y. Vinnikov, G. Srinivasan, J.K. Entin, S.E. Hollinger, N.A. Speranskaya, S. Liu, and A. Namkhai, 2000: The global soil moisture data bank. Bulletin of the American Meteorological Society, 81(6), 1281-1299.

Robson, A.J., T.K. Jones, D.W. Reed, and A.C. Bayliss, 1998: A study of national trend and variation in UK floods. International Journal of Climatology, 18(2), 165-182.

Rocha, A., P. Melo-Goncalves, C. Marques, J. Ferreira, and J.M. Castanheira, 2008: Highfrequency precipitation changes in southeastern Africa due to anthropogenic forcing. International Journal of Climatology, 28(9), 1239-1253.

Rockel, B., and K. Woth, 2007: Extremes of near-surface wind speed over Europe and their future changes as estimated from an ensemble of RCM simulations Climatic Change, 81(S1), 267-280.

Rodda, J.C., M.A. Little, H.J.E. Rodda, and P.E. McSharry, 2010: A comparative study of the magnitude, frequency and distribution of intense rainfall in the United Kingdom. International Journal of Climatology, 30(12), 1776-1783.

Rodier, J.A., and M. Roche, 1984: World Catalogue of Maximum Observed Floods. IAHS Pub. No. 143, IAHS Press, Wallingford, UK

Rodrigo, F.S., 2010: Changes in the probability of extreme daily precipitation observed from 1951 to 2002 in the Iberian Peninsula. International Journal of Climatology, 30(10), 1512-1525

Rodríguez-Puebla, C., A. Encinas, L. García-Casado, and S. Nieto, 2010: Trends in warm days and cold nights over the Iberian Peninsula: relationships to largescale variables. Climatic Change, 100(3), 667-684.

Roer, I., W. Haeberli, and M. Avian, 2008: Observations and considerations on destabilizing active rock glaciers in the European Alps. In: Permafrost on a Warming Planet: Impacts on Ecosystems, Infrastructure and Climate [Kane, D.L. and K.M. Hinkel (eds.)]. Proceedings of the Ninth International Conference on Permafrost at University of Alaska, Fairbanks, 29 June - 03 July 2008, Institute of Northern Engineering, pp 1505-1510.

Roesch, A., 2006: Evaluation of surface albedo and snow cover in AR4 coupled climate models. Journal of Geophysical Research, 111, D15111.

Romanovsky, V.E., D.S. Drozdov, N.G. Oberman, G.V. Malkova, A.L. Kholodov, S.S. Marchenko, N.G. Moskalenko, D.O. Sergeev, N.G. Ukraintseva, A.A. Abramov D.A. Gilichinsky, and A.A. Vasiliev, 2010: Thermal state of permafrost in Russia. Permafrost and Periglacial Processes, 21(2), 136-155.

Ropelewski, C.F., and M.A. Bell, 2008: Shifts in the statistics of daily rainfall in South America conditional on ENSO phase. Journal of Climate, 21(5), 849-865. 
Rosenbluth, B., H.A. Fuenzalida, and P. Aceituno, 1997: Recent temperature variations in southern South America. International Journal of Climatology, 17(1), 67-85.

Rosenzweig, C., G. Casassa, D.J. Karoly, A. Imeson, C. Liu, A. Menzel, S. Rawlins, T.L. Root, B. Seguin, and P. Tryjanowski, 2007: Assessment of Observed Changes and Responses in Natural and Managed Systems. In: Climate Change 2007. Impacts, Adaptation and Vulnerability. Contribution of Working Group II to the Fourth Assessment Report of the Intergovernmental Panel on Climate Change [Parry, M.L., O.F. Canziani, J.P. Palutikof, P.J. Van Der Linden, and C.E. Hanson (eds.)]. Cambridge University Press, Cambridge, UK, pp. 79-131.

Rotstayn, L.D., M.D. Keywood, B.W. Forgan, A.J. Gabric, I.E. Galbally, J.L. Gras, A.K. Luhar, G.H. McTainsh, R.M. Mitchell, and S.A. Young, 2009: Possible impacts of anthropogenic and natural aerosols on Australian climate: A review. International Journal of Climatology, 29(4), 461-479.

Rott, H., S.H. Yueh, D.W. Cline, C. Duguay, R. Essery, C. Haas, F. Heliere, M. Kern, G. Macelloni, E. Malnes, T. Nagler, J. Pulliainen, H. Rebhan, and A. Thompson, 2010: Cold regions hydrology high-resolution observatory for snow and cold land processes. IEEE Proceedings, 98(5), 752-765.

Rowell, D.P. and R.G. Jones, 2006: Causes and uncertainty of future summer drying over Europe. Climate Dynamics, 27(2-3), 281-299.

Ruckstuhl, C. and J.R. Norris, 2009: How do aerosol histories affect solar "dimming" and "brightening" over Europe? IPCC-AR4 models versus observations. Journal of Geophysical Research - Atmospheres, 114, D00D04

Rusticucci, M. and M. Barrucand, 2004: Observed trends and changes in temperature extremes over Argentina. Journal of Climate, 17(20), 4099-4107.

Rusticucci, M. and M. Renom, 2008: Variability and trends in indices of qualitycontrolled daily temperature extremes in Uruguay. International Journal of Climatology, 28(8), 1083-1095.

Rusticucci, M., J. Marengo, O. Penalba, and M. Renom, 2010: An intercomparison of model-simulated in extreme rainfall and temperature events during the last half of the twentieth century. Part 1: mean values and variability. Climate Change, 98(3-4), 493-508.

Ryan, B.F., I.G. Watterson, and J.L. Evans, 1992: Tropical cyclone frequencies inferred from Gray's yearly genesis parameter - Validation of GCM tropical climates. Geophysical Research Letters, 19(18), 1831-1834.

Sailor, D.J., M. Smith, and M. Hart, 2008: Climate change implications for wind power resources in the Northwest United States. Renewable Energy, 33(11), 2393-2406.

Saito, K., M. Kimoto, T. Zhang, K. Takata, and S. Emori, 2007: Evaluating a highresolution climate model: Simulated hydrothermal regimes in frozen ground regions and their change under the global warming scenario. Journal of Geophysical Research - Earth Surface, 112, F02S11.

Santer, B.D., T.M.L. Wigley, P.J. Glecker, C. Bonfils, M.F. Wehner, K. AchutaRoa, T.P. Barnett, J.S. Boyle, W. Brüggemann, M. Fiorino, N. Gillet, J.E. Hansen, P.D. Jones, S.A. Klein, G.A. Meehl, S.C.B. Raper, R.W. Reynolds, K.E. Taylor, and W.M. Washington, 2006: Forced and unforced ocean temperature changes in Atlantic and Pacific tropical cyclogenesis regions. Proceedings of the National Academy of Sciences, 103(38), 13905-13910.

Santer, B.D., C. Mears, F.J. Wentz, K.E. Taylor, P.J. Gleckler, T.M.L. Wigley, T.P. Barnett, J.S. Boyle, W. Bruggemann, N.P. Gillett, S.A. Klein, G.A. Meehl, T. Nozawa, D.W. Pierce, P.A. Stott, W.M. Washington, and M.F. Wehner, 2007: Identification of human-induced changes in atmospheric moisture content. Proceedings of the National Academy of Sciences, 104(39), 15248-15253.

Santos, C.A., and J.I.B. Brito, 2007: Análise dos índices de extremos para o semiárido do Brasil e suas relações com TSM e IVDN. Revista Brasileira de Meteorologia, 22(3), 303-312.

Santos, C.A.C., J. Brito, I, B., T.V.R. Rao, and E.A. Meneses, 2009: Tendências dos Índices de precipitação no Estado do Ceará. Revista Brasileira de Meteorologia, 24(1), 39-47.

Sasaki, W., and H. Toshiyuki, 2007: Interannual variability and predictability of summertime significant wave heights in the western North Pacific. Journal of Oceanography, 63(2), 203-213.

Sauber, J.M., and B.F. Molnia, 2004: Glacier ice mass fluctuations and fault instability in tectonically active Southern Alaska. Global and Planetary Change, 42(1-4), 279-293.
Sauber, J.M., and N.A. Ruppert, 2008: Rapid ice mass loss: does it have an influence on earthquake occurrence in southern Alaska? In: Active Tectonics and Seismic Potential of Alaska [Freymueller, J.T., P.J. Haeussler, R. Wesson, and G. Ekström (eds.)]. American Geophysical Union, Washington, DC.

Scaife, A.A., C.K. Folland, L.V. Alexander, A. Moberg, and J.R. Knight, 2008: European climate extremes and the North Atlantic Oscillation. Journal of Climate, 21(1), 72-83.

Scaife, A.A., F. Kucharski, C.K. Folland, J. Kinter, S. Bronnimann, D. Fereday, A.M. Fischer, S. Grainger, E.K. Jin, I.S. Kang, J.R. Knight, S. Kusunoki, N.C. Lau, M.J. Nath, T. Nakaegawa, P. Pegion, S. Schubert, P. Sporyshev, J. Syktus, J.H. Yoon, N. Zeng, and T. Zhou, 2009: The CLIVAR C20C project: selected twentieth century climate events. Climate Dynamics, 33(5), 603-614.

Scaife, A.A., T. Spangehl, D. Fereday, U. Cubasch, U. Langematz, H. Akiyoshi, S. Bekki, P. Braesicke, N. Butchart, M. Chipperfield, A. Gettelman, S. Hardiman, M. Michou, E. Rozanov, and T. Shepherd, 2011: Climate change projections and stratosphere-troposphere interaction. Climate Dynamics, doi:10.1007/s0038200011-01080-00387.

Schaefer, K., T. Zhang, L. Bruhwiler, and A.P. Barrett, 2011: Amount and timing of permafrost carbon release in response to climate warming. Tellus Series $B-$ Chemical and Physical Meteorology, 63(2), 165-180.

Schär, C., and G. Jendritzky, 2004: Climate change: Hot news from summer 2003. Nature, 432(7017), 559-560.

Schär, C., P.L. Vidale, D. Lüthi, C. Frei, C. Häberli, M.A. Liniger, and C. Appenzeller, 2004: The role of increasing temperature variability in European summer heatwaves. Nature, 427(322), 332-336.

Scheffer, M., J. Bascompte, W.A. Brock, V. Brovkin, S.R. Carpenter, V. Dakos, H. Held, E.H. van Nes, M. Rietkerk, and G. Sugihara, 2009: Early-warning signals for critical transitions. Nature, 461(7260), 53-59.

Schiefer, E., B. Menounos, and R. Wheate, 2007: Recent volume loss of British Columbian glaciers, Canada. Geophysical Research Letters, 34, L16503.

Schmidli, J., and C. Frei, 2005: Trends of heavy precipitation and wet and dry spells in Switzerland during the 20th century. International Journal of Climatology, 25(6), 753-771.

Schmidli, J., C.M. Goodess, C. Frei, M.R. Haylock, Y. Hundecha, J. Ribalaygua, and T. Schmith, 2007: Statistical and dynamical downscaling of precipitation: An evaluation and comparison of scenarios for the European Alps. Journal of Geophysical Research - Atmospheres, 112, D04105.

Schneidereit, A., R. Blender, K. Fraedrich, and F. Lunkeit, 2007: Icelandic climate and north Atlantic cyclones in ERA-40 reanalyses. Meteorologische Zeitschrift, 16(1), 17-23.

Schölzel, C., and P. Friederichs, 2008: Multivariate non-normally distributed random variables in climate research - introduction to the copula approach. Nonlinear Processes in Geophysics, 15(5), 761-772.

Schubert, S.D., M.J. Suarez, P.J. Pegion, R.D. Koster, and J.T. Bacmeister, 2004: On the cause of the 1930s Dust Bowl. Science, 303(5665), 1855-1859.

Schubert, S.D., Y. Chang, M.J. Suarez, and P.J. Pegion, 2008a: ENSO and wintertime extreme precipitation events over the contiguous united states. Journal of Climate, 21(1), 22-39.

Schubert, S.D., M.J. Suarez, P.J. Pegion, R.D. Koster, and J.T. Bacmeister, 2008b: Potential predictability of long-term drought and pluvial conditions in the US Great Plains. Journal of Climate, 21(4), 802-816.

Schubert, S.D., D. Gutzler, H.L. Wang, A. Dai, T. Delworth, C. Deser, K. Findell, R. Fu, W. Higgins, M. Hoerling, B. Kirtman, R. Koster, A. Kumar, D. Legler, D. Lettenmaier, B. Lyon, V. Magana, K. Mo, S. Nigam, P. Pegion, A. Phillips, R. Pulwarty, D. Rind, A. Ruiz-Barradas, J. Schemm, R. Seager, R. Stewart, M. Suarez, J. Syktus, M.F. Ting, C.Z. Wang, S. Weaver, and N. Zeng, 2009: A US CLIVAR project to assess and compare the responses of global climate models to drought-related SST forcing patterns: Overview and results. Journal of Climate, 22(19), 5251-5272.

Scileppi, E., and J.P. Donnelly, 2007: Sedimentary evidence of hurricane strikes in western Long Island, New York. Geochemistry Geophysics Geosystems, 8, Q06011.

Scott, K.M., J.W. Vallance, N. Kerle, J. Luis Macías, W. Strauch, and G. Devoli, 2005: Catastrophic precipitation-triggered lahar at Casita volcano, Nicaragua: occurrence, bulking and transformation. Earth Surface Processes and Landforms, 30(1), 59-79. 
Seager, R., M.F. Ting, I. Held, Y. Kushnir, J. Lu, G. Vecchi, H.P. Huang, N. Harnik, A. Leetmaa, N.C. Lau, C.H. Li, J. Velez, and N. Naik, 2007: Model projections of an imminent transition to a more arid climate in southwestern North America. Science, 316(5828), 1181-1184.

Seager, R., A. Tzanova, and J. Nakamura, 2009: Drought in the southeastern United States: Causes, variability over the last millennium, and the potential for future hydroclimate change. Journal of Climate, 22(19), 5021-5045.

Seierstad, I.A., D.B. Stephenson, and N.G. Kvamsto, 2007: How useful are teleconnection patterns for explaining variability in extratropical storminess? Tellus Series A - Dynamic Meteorology and Oceanography, 59(2), 170-181.

Seleshi, Y. and P. Camberlin, 2006: Recent changes in dry spell and extreme rainfall events in Ethiopia. Theoretical and Applied Climatology, 83(1-4), 181-191.

Semenov, M.A., 2008: Simulation of extreme weather events by a stochastic weather generator. Climate Research, 35(3), 203-212.

Semmler, T., S. Varghese, R. McGrath, P. Nolan, S. Wang, P. Lynch, and C. O'Dowd, 2008: Regional model simulation of North Atlantic cyclones: Present climate and idealized response to increased sea surface temperature. Journal of Geophysical Research - Atmospheres, 113, D02107.

Sen Roy, S., 2009: A spatial analysis of extreme hourly precipitation patterns in India. International Journal of Climatology, 29(3), 345-355.

Seneviratne, S.I., D. Lüthi, M. Litschi, and C. Schär, 2006a: Land-atmosphere coupling and climate change in Europe. Nature, 443(7108), 205-209.

Seneviratne, S.I., R.D. Koster, Z.C. Guo, P.A. Dirmeyer, E. Kowalczyk, D. Lawrence, P. Liu, C.H. Lu, D. Mocko, K.W. Oleson, and D. Verseghy, 2006b: Soil moisture memory in AGCM simulations: Analysis of global land-atmosphere coupling experiment (GLACE) data. Journal of Hydrometeorology, 7(5), 1090-1112.

Seneviratne, S.I., T. Corti, E.L. Davin, M. Hirschi, E. Jaeger, I. Lehner, B. Orlowsky, and A.J. Teuling, 2010: Investigating soil moisture-climate interactions in a changing climate: A review. Earth Science Reviews, 99(3-4), 125-161.

Sepp, M., and J. Jaagus, 2011: Changes in the activity and tracks of Arctic cyclones. Climatic Change, 105(3-4), 577-595.

Shanahan, T.M., J.T. Overpeck, K.J. Anchukaitis, J.W. Beck, J.E. Cole, D.L. Dettman, J.A. Peck, C.A. Scholz, and J.W. King, 2009: Atlantic forcing of persistent drought in West Africa. Science, 324(5925), 377-380.

Shao, Y., and C.H. Dong, 2006: A review on East Asian dust storm climate, modelling and monitoring. Global and Planetary Change, 52(1-4), 1-22.

Shaw, S.B., and S.J. Riha, 2011: Assessing temperature-based PET equations under a changing climate in temperate, deciduous forests. Hydrological Processes, 25(9), 1466-1478.

Sheffield, J, and E.F. Wood, 2008a: Global trends and variability in soil moisture and drought characteristics, 1950-2000, from observation-driven simulations of the terrestrial hydrologic cycle. Journal of Climate, 21(3), 432-458.

Sheffield, J., and E.F. Wood, 2008b: Projected changes in drought occurrence under future global warming from multi-model, multi-scenario, IPCC AR4 simulations. Climate Dynamics, 31(1), 79-105.

Sheppard, C., D.J. Dixon, M. Gourlay, A. Sheppard, and R. Payet, 2005: Coral mortality increases wave energy reaching shores protected by reef flats: Examples from the Seychelles. Estuarine Coastal and Shelf Science, 64(2-3), 223-234.

Sherwood, S.C., and M. Huber, 2010: An adaptability limit to climate change due to heat stress. Proceedings of the National Academy of Sciences, 107(21), 9552-9555.

Shiklomanov, A.I., R.B. Lammers, M.A. Rawlins, L.C. Smith, and T.M. Pavelsky, 2007: Temporal and spatial variations in maximum river discharge from a new Russian data set. Journal of Geophysical Research - Biogeosciences, 112, G04S53.

Shiklomanov, N.I., D.A. Streletskiy, F.E. Nelson, R.D. Hollister, V.E. Romanovsky, C.E. Tweedie, J.G. Bockheim, and J. Brown, 2010: Decadal variations of active-layer thickness in moisture-controlled landscapes, Barrow, Alaska. Journal of Geophysical Research - Biogeosciences, 115, G00I04.

Shongwe, M.E., G.J. van Oldenborgh, B.J.J.M. van den Hurk, B. de Boer, C.A.S. Coelho, and M.K. van Aalst, 2009: Projected changes in mean and extreme precipitation in Africa under global warming. Part I: Southern Africa. Journal of Climate, 22(13), 3819-3837.
Shongwe, M.E., G.J. van Oldenborgh, B.J.J.M. van den Hurk, B. de Boer, C.A.S Coelho, and M.K. van Aalst, 2011: Projected changes in mean and extreme precipitation in Africa under global warming. Part II: East Africa. Journal of Climate, 24(14), 3718-3732.

Shukla, J., 2007: Monsoon mysteries. Science, 318(5848), 204-205.

Shulmeister, J., D.T. Rodbell, M.K. Gagan, and G.O. Seltzer, 2006: Inter-hemispheric linkages in climate change: paleo-perspectives for future climate change. Climates of the Past, 2(2), 167-185.

Sidle, R.C., and H. Ochiai, 2006: Landslides: processes, prediction, and land use. Water Resources Monograph 18, American Geophysical Union, Washington, DC, 312 pp.

Sigmundsson, F., V. Pinel, B. Lund, F. Albino, C. Pagli, H. Geirsson, and E. Sturkell, 2010: Climate effects on volcanism: influence on magmatic systems of loading and unloading from ice mass variations, with examples from Iceland. Philosophical Transactions of the Royal Society A, 368(1919), 2519-2534.

Sillmann, J. and E. Roeckner, 2008: Indices for extreme events in projections of anthropogenic climate change. Climatic Change, 86(1-2), 83-104.

Silva, A.G., and P. Azevedo, 2008: Índices de tendências de Mudanças Climáticas no Estado da Bahia. Engenheiria Ambiental, 5, 141-151.

Silva Dias, M.A.F., S. Rutledge, P. Kabat, P.L. Silva Dias, C. Nobre, G. Fisch, A.J. Dolman, E. Zipser, M. Garstang, A.O. Manzi, J.D. Fuentes, H.R. Rocha, J. Marengo, A. Plana-Fattori, L.D.A. Sá, R.C.S. Alvalá, M.O. Andreae, P. Artaxo, R. Gielow, and L. Gatti, 2002: Cloud and rain processes in a biosphere-atmosphere interaction context in the Amazon Region. Journal of Geophysical Research -Atmospheres, 107(D20), 8072.

Silvestri, G.E. and C.S. Vera, 2009: Nonstationary impacts of the Southern Annular Mode on Southern Hemisphere climate. Journal of Climate, 22(22), 6142-6148.

Simmonds, I. and K. Keay, 2002: Surface fluxes of momentum and mechanical energy over the North Pacific and North Atlantic Oceans. Meteorology and Atmospheric Physics, 80(1), 1-18

Simmonds, I., C. Burke, and K. Keay, 2008: Arctic climate change as manifest in cyclone behavior. Journal of Climate, 21(22), 5777-5796.

Simmons, A.J., K.M. Willett, P.D. Jones, P.W. Thorne, and D.P. Dee, 2010: Low-frequency variations in surface atmospheric humidity, temperature, and precipitation: Inferences from reanalyses and monthly gridded observational data sets. Journal of Geophysical Research - Atmospheres, 115, D01110.

Sinha, A., K.G. Cannariato, L.D. Stott, H. Cheng, R.L. Edwards, M.G. Yadava, R. Ramesh, and I.B. Singh, 2007: A 900-year (600 to 1500 A. D.) record of the Indian summer monsoon precipitation from the core monsoon zone of India. Geophysical Research Letters, 34, L16707.

Sinton, J., K. Grönvold, and K. Sæmundsson, 2005: Postglacial eruptive history of the Western Volcanic Zone, Iceland. Geochemistry Geophysics Geosystems, $\mathbf{6}$ Q12009.

Sjogren, P., 2009: Sand mass accumulation rate as a proxy for wind regimes in the SW Barents Sea during the past 3 ka. Holocene, 19(4), 591-598.

Small, I., J. van der Meer, and R.E.G., Upshur, 2001: Acting on an environmental health disaster: The case of the Aral Sea. Environmental Health Perspectives, 109(6), 547-549.

Smith, J.M., M.A. Cialone, T.V. Wamsley, and T.O. McAlpin, 2010: Potential impact of sea level rise on coastal surges in southeast Louisiana. Ocean Engineering, 37(1), 37-47.

Smith, K., and R. Ward, 1998: Floods. Physical Processes and Human Impacts. John Wiley, Chichester, UK, 382 pp.

Smith, L.C., 2000: Trends in Russian Arctic river-ice formation and breakup, 1917 to 1994. Physical Geography, 21(1), 46-56.

Smith, L.C., Y. Sheng, G.M. MacDonald, and L.D. Hinzman, 2005: Disappearing Arctic lakes. Science, 308(5727), 1429

Smith, S.L., S.A. Wolfe, D.W. Riseborough, and F.M. Nixon, 2009: Active-layer characteristics and summer climatic indices, Mackenzie Valley, Northwest Territories, Canada. Permafrost and Periglacial Processes, 20(2), 201-220.

Smith, S.L., V.E. Romanovsky, A.G. Lewkowicz, C.R. Burn, M. Allard, G.D. Clow, K. Yoshikawa, and J. Throop, 2010: Thermal state of permafrost in North America: a contribution to the international polar year. Permafrost and Periglacial Processes, 21(2), 117-135. 
Smits, A., A.M.G.K. Tank, and G.P. Konnen, 2005: Trends in storminess over the Netherlands, 1962-2002. International Journal of Climatology, 25(10), 1331-1344.

Soares, W.R., and J.A. Marengo, 2009: Assessments of moisture fluxes east of the Andes in South America in a global warming scenario. International Journal of Climatology, 29(10), 1395-1414.

Son, S.W., L.M. Polvani, D.W. Waugh, H. Akiyoshi, R. Garcia, D. Kinnison, S. Pawson, E. Rozanov, T.G. Shepherd, and K. Shibata, 2008: The impact of stratospheric ozone recovery on the Southern Hemisphere westerly jet. Science, 320(5882), 1486-1489.

Son, S.W., E.P. Gerber, J. Perlwitz, L.M. Polvani, N.P. Gillett, K.H. Seo, V. Eyring, T.G. Shepherd, D. Waugh, H. Akiyoshi, J. Austin, A. Baumgaertner, S. Bekki, P. Braesicke, C. Bruhl, N. Butchart, M.P. Chipperfield, D. Cugnet, M. Dameris, S. Dhomse, S. Frith, H. Garny, R. Garcia, S.C. Hardiman, P. Jockel, J.F. Lamarque, E. Mancini, M. Marchand, M. Michou, T. Nakamura, O. Morgenstern, G. Pitari, D.A. Plummer, J. Pyle, E. Rozanov, J.F. Scinocca, K. Shibata, D. Smale, H. Teyssedre, W. Tian, and Y. Yamashita, 2010: Impact of stratospheric ozone on Southern Hemisphere circulation change: A multimodel assessment. Journal of Geophysical Research-Atmospheres, 115, D00M07.

Sørensen, L.S., S.B. Simonsen, K. Nielsen, P. Lucas-Picher, G. Spada, G. Adalgeirsdottir, R. Forsberg, and C.S. Hvidberg, 2011: Mass balance of the Greenland ice sheet (2003-2008) from ICESat data - the impact of interpolation, sampling and firn density. The Cryosphere, 5(1), 173-186.

Sorensson, A.A., C.G. Menéndez, R. Ruscica, P. Alexander, P. Samuelsson, and U. Willén, 2010: Projected precipitation changes in South America: a dynamical downscaling within CLARIS. Meteorologische Zeitschrift, 19(4), 347-355.

Sorteberg, A. and J.E. Walsh, 2008: Seasonal cyclone variability at 70 degrees $\mathrm{N}$ and its impact on moisture transport into the Arctic. Tellus Series $A$ - Dynamic Meteorology and Oceanography, 60(3), 570-586.

Sosio, R., G.B. Crosta, and O. Hungr, 2008: Complete dynamic modeling calibration for the Thurwieser rock avalanche (Italian Central Alps) Engineering Geology, 100(1-2), 11-26.

Stahl, K., H. Hisdal, J. Hannaford, L.M. Tallaksen, H.A.J. van Lanen, E. Sauquet, S. Demuth, M. Fendekova, and J. Jódar, 2010: Streamflow trends in Europe: evidence from a dataset of near-natural catchments. Hydrology and Earth System Sciences Discussion, 7(4), 5769-5804.

Stephenson, D.B., H.F. Diaz, and R.J. Murnane, 2008: Definition, diagnosis, and origin of extreme weather and climate events. In: Climate Extremes and Society [Murnane, R.J. and H.F. Diaz (eds.)]. Cambridge University Press, Cambridge, UK, pp. 11-23.

Stephenson, T.S., C.M. Goodess, M.R. Haylock, A.A. Chen, and M.A. Taylor, 2008: Detecting inhomogeneities in Caribbean and adjacent Caribbean temperature data using sea-surface temperatures. Journal of Geophysical Research Atmospheres, 113, D21116

Sterl, A., C. Severijns, H. Dijkstra, W. Hazeleger, G.J. van Oldenborgh, M. van den Broeke, G. Burgers, B. van den Hurk, P.J. van Leeuwen, and P. van Velthoven, 2008: When can we expect extremely high surface temperatures? Geophysical Research Letters, 35, L14703.

Sterl, A., H. van den Brink, H. de Vries, R. Haarsma, and E. van Meijgaard, 2009: An ensemble study of extreme storm surge related water levels in the North Sea in a changing climate. Ocean Science, 5(3), 369-378.

Sterr, H., 2008: Assessment of vulnerability and adaptation to sea-level rise for the coastal zone of Germany. Journal of Coastal Research, 24(2), 380-393.

Stewart, I.S., J. Sauber, and J. Rose, 2000: Glacio-seismotectonics: ice sheets, crustal deformation and seismicity. Quaternary Science Reviews, 19(14-15), 1367-1389.

Stive, M.J.F., Z.B. Wang, and C. Lakhan, 2003: Morphodynamic modeling of tidal basins and coastal inlets. In: Advances in Coastal Modeling [Lakhan, V.C. (ed.)]. Elsevier, Amsterdam, The Netherlands, pp. 367-392.

Stoffel, M., I. Lièvre, D. Conus, M.A. Grichting, H. Raetzo, H.W. Gärtner, and M. Monbaron, 2005: 400 years of debris-flow activity and triggering weather conditions: Ritigraben, Valais, Switzerland. Arctic, Antarctic, and Alpine Research, 37(3), 387-395.

Stott, P.A., D.A. Stone, and M.R. Allen, 2004: Human contribution to the European heatwave of 2003. Nature, 432(7017), 610-614.
Stott, P.A., N.P. Gillett, G.C. Hegerl, D. Karoly, D. Stone, X. Zhang, and F.W. Zwiers, 2010: Detection and attribution of climate change: a regional perspective. Wiley Interdisciplinary Reviews: Climate Change, 1(2), 192-211.

Su, B., Z.W. Kundzewicz, and T. Jiang, 2009: Simulation of extreme precipitation over the Yangtze River Basin using Wakeby distribution. Theoretical and Applied Climatology, 96(3-4), 209-219.

Sugahara, S., R.P. da Rocha, and R. Silveira, 2009: Non-stationary frequency analysis of extreme daily rainfall in Sao Paulo, Brazil. International Journal of Climatology, 29(9), 1339-1349.

Sugi, M., A. Noda, and N. Sato, 2002: Influence of the global warming on tropical cyclone climatology: An experiment with the JMA global model. Journal of the Meteorological Society of Japan, 80(2), 249-272.

Sugi, M., H. Murakami, and J. Yoshimura, 2009: A reduction in global tropical cyclone frequency due to global warming. SOLA, 5, 164-167.

Sugiyama, M., H. Shiogama, and S. Emori, 2010: Precipitation extreme changes exceeding moisture content increases in MIROC and IPCC climate models. Proceedings of the National Academy of Sciences, 107(2), 571-575.

Suppiah, R., K. Hennessy, P.H. Whetton, K. Mclnnes, I. Macadam, J. Bathols, J. Ricketts, and C.M. Page, 2007: Australian climate change projections derived from simulations performed for the IPCC 4th Assessment Report. Australian Meteorological Magazine, 56(3), 131-152.

Suter, S., M. Laternser, W. Haeberli, R. Frauenfelder, and M. Hoelzle, 2001: Cold firn and ice of high-altitude glaciers in the Alps: measurements and distribution modelling. Journal of Glaciology, 47(156), 85-96.

Sutton, R.T., B. Dong, and J.M. Gregory, 2007: Land/sea warming ratio in response to climate change: IPCC AR4 model results and comparison with observations. Geophysical Research Letters, 34, L02701.

Suursaar, U., and T. Kullas, 2009: Decadal variations in wave heights off Cape Kelba, Saaremaa Island, and their relationships with changes in wind climate. Oceanologia, 51(1), 39-61.

Svensson, C., and D.A. Jones, 2002: Dependence between extreme sea surge, river flow and precipitation in eastern Britain. International Journal of Climatology, 22(10), 1149-1168

Swanson, K.L., 2008: Non-locality of Atlantic tropical cyclone intensities. Geochemistry Geophysics Geosystems, 9, Q04V01.

Syvitski, J.P.M., A.J. Kettner, I. Overeem, E.W.H. Hutton, M.T. Hannon, G.R. Brakenridge, J. Day, C. Vorosmarty, Y. Saito, L. Giosan, and R.J. Nicholls, 2009: Sinking deltas due to human activities. Nature Geoscience, 2(10), 681-686.

Tallaksen, L.M., H. Hisdal, and H.A.J. Van Lanen, 2009: Space-time modelling of catchment scale drought characteristics. Journal of Hydrology, 375(3-4), 363-372.

Tamura, T., K. Horaguchi, Y. Saito, L.N. Van, M. Tateishi, K.O.T. Thi, F. Nanayama, and K. Watanabe, 2010: Monsoon-influenced variations in morphology and sediment of a mesotidal beach on the Mekong River delta coast. Geomorphology, 116(1-2), 11-23.

Tanaka, H.L., N. Ishizaki, and D. Nohara, 2005: Intercomparison of the intensities and trends of Hadley, Walker and monsoon circulations in the global warming projections. SOLA, 1, 77-80.

Taschetto, A.S., C.C. Ummenhofer, A. Sen Gupta, and M.H. England, 2009: The effect of anomalous warming in the central Pacific on the Australian monsoon. Geophysical Research Letters, 36, L12704.

Taye, M.T., V. Ntegeka, N.P. Ogiramoi, and P. Willems, 2011: Assessment of climate change impact on hydrological extremes in two source regions of the Nile River Basin. Hydrology and Earth System Sciences, 15(1), 209-222.

Taylor, A.E., K. Wang, S.L. Smith, M.M. Burgess, and A.S. Judge, 2006: Canadian Arctic Permafrost Observatories: Detecting contemporary climate change through inversion of subsurface temperature time series. Journal of Geophysical Research - Solid Earth, 111, B02411.

Tebaldi, C. and R. Knutti, 2007: The use of the multi-model ensemble in probabilistic climate projections. Philosophical Transactions of the Royal Society A, 365(1857), 2053-2075

Tebaldi, C. and B. Sanso, 2009: Joint projections of temperature and precipitation change from multiple climate models: a hierarchical Bayesian approach. Journal of the Royal Statistical Society Series A - Statistics in Society, 172(1), 83-106. 
Tebaldi, C., K. Hayhoe, J.M. Arblaster, and G.A. Meehl, 2006: Going to the extremes. An intercomparison of model-simulated historical and future changes in extreme events. Climatic Change, 79(3-4), 185-211.

Teng, H., W.M. Washington, and G.A. Meehl, 2008: Interannual variations and future change of wintertime extratropical cyclone activity over North America in CCSM3. Climate Dynamics, 30(7-8), 673-686.

Teuling, A.J., M. Hirschi, A. Ohmura, M. Wild, M. Reichstein, P. Ciais, N. Buchmann, C. Ammann, L. Montagnani, A.D. Richardson, G. Wohlfahrt, and S.I. Seneviratne, 2009: A regional perspective on trends in continental evaporation. Geophysical Research Letters, 36, L02404.

Teuling, A.J., S.I. Seneviratne, R. Stockli, M. Reichstein, E. Moors, P. Ciais, S. Luyssaert, B. van den Hurk, C. Ammann, C. Bernhofer, E. Dellwik, D. Gianelle, B. Gielen, T. Grunwald, K. Klumpp, L. Montagnani, C. Moureaux, M. Sottocornola, and G. Wohlfahrt, 2010: Contrasting response of European forest and grassland energy exchange to heatwaves. Nature Geoscience, 3(10), 722-727.

Textor, C., M. Schulz, S. Guibert, S. Kinne, Y. Balkanski, S. Bauer, T. Berntsen, T. Berglen, O. Boucher, M. Chin, F. Dentener, T. Diehl, R. Easter, H. Feichter, D. Fillmore, S. Ghan, P. Ginoux, S. Gong, J.E. Kristjansson, M. Krol, A. Lauer, J.F. Lamarque, X. Liu, V. Montanaro, G. Myhre, J. Penner, G. Pitari, S. Reddy, O. Seland, P. Stier, T. Takemura, and X. Tie, 2006: Analysis and quantification of the diversities of aerosol life cycles within AeroCom. Atmospheric Chemistry and Physics, 6(7), 1777-1813.

ThemeßI, M.J., A. Gobiet, and A. Leuprecht, 2011: Empirical-statistical downscaling and error correction of daily precipitation from regional climate models. International Journal of Climatology, 31(10), 1530-1544, doi: $10.1002 /$ joc.2168.

Thodsen, H., 2007: The influence of climate change on stream flow in Danish rivers. Journal of Hydrology, 333(2-4), 226-238.

Thomas, D.S.G., M. Knight, and G.F.S. Wiggs, 2005: Remobilization of southern African desert dune systems by twenty-first century global warming. Nature, 435(7046), 1218-1221.

Thompson, L.G., E. Mosley-Thompson, H. Brecher, M. Davis, B. Leon, D. Les, P.N. Lin, T. Mashiotta, and K. Mountain, 2006: Abrupt tropical climate change: Past and present. Proceedings of the National Academy of Sciences, 103(28), 10536-10543.

Tietsche, S., D. Notz, J.H. Jungclaus, and J. Marotzke, 2011: Recovery mechanisms of Arctic summer sea ice. Geophysical Research Letters, 38, L02707.

Timbal, B., P. Hope, and S. Charles, 2008: Evaluating the consistency between statistically downscaled and global dynamical model climate change projections. Journal of Climate, 21(22), 6052-6059.

Timbal, B., E. Fernandez, and Z. Li, 2009: Generalization of a statistical downscaling model to provide local climate change projections for Australia. Environmental Modelling \& Software, 24(3), 341-358.

Timmermann, A., S. McGregor, and F.F. Jin, 2010: Wind effects on past and future regional sea level trends in the southern Indo-Pacific. Journal of Climate, 23(16), 4429-4437.

Tolika, K., P. Maheras, and I. Tegoulias, 2009: Extreme temperatures in Greece during 2007: Could this be a "return to the future"? Geophysical Research Letters, 36, L10813.

Tomassini, L. and D. Jacob, 2009: Spatial analysis of trends in extreme precipitation events in high-resolution climate model results and observations for Germany. Journal of Geophysical Research - Atmospheres, 114, D12113.

Toreti, A. and F. Desiato, 2008: Changes in temperature extremes over Italy in the last 44 years. International Journal of Climatology, 28(6), 733-745.

Toreti, A., E. Xoplaki, D. Maraun, F.G. Kuglitsch, H. Wanner, and J. Luterbacher, 2010: Characterisation of extreme winter precipitation in Mediterranean coastal sites and associated anomalous atmospheric circulation patterns. Natural Hazards and Earth System Sciences, 10(5), 1037-1050.

Trenberth, K.E., D. Stepaniak, J. Hurrell, and M. Fiorino, 2001: Quality of reanalyses in the tropics. Journal of Climate, 14(7), 1499-1510.

Trenberth, K.E., J. Fasullo, and L. Smith, 2005: Trends and variability in column-integrated atmospheric water vapor. Climate Dynamics, 24(7-8), 741-758.
Trenberth, K.E., P.D. Jones, P. Ambenje, R. Bojariu, D. Easterling, A. Klein Tank, D. Parker, F. Rahimzadeh, J.A. Renwick, M. Rusticucci, B. Solden, and P. Zhai, 2007: Observations: Surface and atmospheric climate change. In: Climate Change 2007: The Physical Science Basis. Contribution of Working Group I to the Fourth Assessment Report of the Intergovernmental Panel on Climate Change [Solomon, S., D. Qin, M. Manning, Z. Chen, M. Marquis, K.B. Averyt, M. Tignor and H.L. Miller (eds.)]. Cambridge University Press, Cambridge, UK, and New York, NY, pp. 235-336.

Trewin, B. and H. Vermont, 2010: Changes in the frequency of record temperatures in Australia, 1957-2009. Australian Meteorological and Oceanographic Journal, 60(2), 113-119.

Trigo, I.F., 2006: Climatology and interannual variability of storm-tracks in the EuroAtlantic sector: a comparison between ERA-40 and NCEP/NCAR reanalyses. Climate Dynamics, 26(2-3), 127-143.

Trömel, S. and C.D. Schönwiese, 2005: A generalized method of time series decomposition into significant components including probability assessments of extreme events and application to observed German precipitation data. Meteorologische Zeitschrift, 14, 417-427.

Trouet, V., J. Esper, N.E. Graham, A. Baker, J.D. Scourse, and D.C. Frank, 2009: Persistent positive North Atlantic Oscillation mode dominated the medieval climate anomaly. Science, 324(5923), 78-80.

Tsimplis, M.N., M. Marcos, and S. Somot, 2008: 21st century Mediterranean sea level rise: Steric and atmospheric pressure contributions from a regional model. Global and Planetary Change, 63(2-3), 105-111.

Tu, M., M.J. Hall, P.J.M. de Laat, and M.J.M. de Wit, 2005: Extreme floods in the Meuse river over the past century: aggravated by land-use changes? Physics and Chemistry of the Earth, 30(4-5), 267-276.

Tudhope, A.W., C.P. Chilcott, M.T. McCulloch, E.R. Cook, J. Chappell, R.M. Ellam, D.W. Lea, J.M. Lough, and G.B. Shimmield, 2001: Variability in the El Niño-Southern Oscillation through a glacial-interglacial cycle. Science, 291(5508), 1511-1517.

Tuffen, H., 2010: How will melting of ice affect volcanic hazards in the twenty-first century? Philosophical Transactions of the Royal Society A, 368(1919), 2535-2558.

Turner, A.G. and J.M. Slingo, 2009: Subseasonal extremes of precipitation and activebreak cycles of the Indian summer monsoon in a climate-change scenario. Quarterly Journal of the Royal Meteorological Society, 135(640), 549-567.

Turner, J., S.R. Colwell, G.J. Marshall, T.A. Lachlan-Cope, A.M. Carleton, P.D. Jones, V. Lagun, P.A. Reid, and S. lagovkina, 2005: Antarctic climate change during the last 50 years. International Journal of Climatology, 25(3), 279-294.

Turpeinen, H., A. Hampel, T. Karow, and G. Maniatis, 2008: Effect of ice sheet growth and melting on the slip evolution of thrust faults. Earth and Planetary Science Letters, 269(1-2), 230-241.

Uchiyama, T., R. Mizuta, K. Kamiguchi, A. Kitoh, and A. Noda, 2006: Changes in temperature-based extremes indices due to global warming projected by a global 20-km-mesh atmospheric model. SOLA, 2, 68-71.

Ulbrich, U., J.G. Pinto, H. Kupfer, G.C. Leckebusch, T. Spangehl, and M. Reyers, 2008: Changing northern hemisphere storm tracks in an ensemble of IPCC climate change simulations. Journal of Climate, 21(8), 1669-1679.

Ulbrich, U., G.C. Leckebusch, and J.G. Pinto, 2009: Extra-tropical cyclones in the present and future climate: a review. Theoretical and Applied Climatology, 96(1-2), 117-131.

Ullmann, A., P.A. Pirazzoli, and A. Tomasin, 2007: Sea surges in Camargue: Trends over the 20th century. Continental Shelf Research, 27(7), 922-934.

Ullmann, A., P.A. Pirazzoli, and V. Moron, 2008: Sea surges around the Gulf of Lions and atmospheric conditions. Global and Planetary Change, 63(2-3), 203-214.

Ummenhofer, C.C., A. Sen Gupta, M.J. Pook, and M.H. England, 2008: Anomalous rainfall over southwest Western Australia forced by Indian Ocean sea surface temperatures. Journal of Climate, 21(19), 5113-5134.

Ummenhofer, C.C., A. Sen Gupta, M.H. England, and C.J.C. Reason, 2009a: Contributions of Indian Ocean sea surface temperatures to enhanced East African rainfall. Journal of Climate, 22(4), 993-1013.

Ummenhofer, C.C., A. Sen Gupta, A.S. Taschetto, and M.H. England, 2009b: Modulation of Australian precipitation by meridional gradients in East Indian Ocean sea surface temperature. Journal of Climate, 22(21), 5597-5610. 
Ummenhofer, C.C., M.H. England, G.A. Meyers, P.C. McIntosh, M.J. Pook, J.S. Risbey, A. Sen Gupta, and A.S. Taschetto, 2009c: What causes Southeast Australia's worst droughts? Geophysical Research Letters, 36, L04706.

Vallée, S. and S. Payette, 2007: Collapse of permafrost mounds along a subarctic river over the last 100 years (northern Québec). Geomorphology, 90(1-2), 162-170.

Vallve, M.B., and J. Martin-Vide, 1998: Secular climatic oscillations as indicated by catastrophic floods in the Spanish Mediterranean coastal area (14th-19th centuries). Climatic Change, 38(4), 473-491.

Van den Brink, H.W., G.P. Konnen, J.D. Opsteegh, G.J. Van Oldenborgh, and G. Burgers, 2005: Estimating return periods of extreme events from ECMWF seasonal forecast ensembles. International Journal of Climatology, 25(10), 1345-1354.

van den Hurk, B.J.J.M., and E. van Meijgaard, 2010: Diagnosing land-atmosphere interaction from a Regional Climate Model simulation over West Africa. Journal of Hydrometeorology, 11(2), 467-481.

van der Linden, P. and J.F.B. Mitchell (eds.), 2009: ENSEMBLES: Climate Change and its Impacts: Summary of research and results from the ENSEMBLES project. Met Office Hadley Centre, Exeter, UK, $160 \mathrm{pp}$.

van der Schrier, G., K.R. Briffa, T.J. Osborn, and E.R. Cook, 2006a: Summer moisture availability across North America. Journal of Geophysical Research Atmospheres, 111, D11102.

van der Schrier, G., K.R. Briffa, P.D. Jones, and T.J. Osborn, 2006b: Summer moisture variability across Europe. Journal of Climate, 19(12), 2818-2834.

van Oldenborgh, G.J., S. Philip, and M. Collins, 2005: El Niño in a changing climate: a multi-model study. Ocean Science, 1(2), 267-298.

van Oldenborgh, G.J., S. Drijfhout, A. van Ulden, R. Haarsma, A. Sterl, C. Severijns, W. Hazeleger, and H. Dijkstra, 2009: Western Europe is warming much faster than expected. Climate of the Past, 5(1), 1-12.

van Pelt, S.C., P. Kabat, H.W. ter Maat, B.J.J.M. van den Hurk, and A.H. Weerts, 2009: Discharge simulations performed with a hydrological model using bias corrected regional climate model input. Hydrology and Earth System Sciences, 13(12), 2387-2397.

Vautard, R., P. Yiou, F. D'Andrea, N. de Noblet, N. Viovy, C. Cassou, J. Polcher, P. Ciais, M. Kageyama, and Y. Fan, 2007: Summertime European heat and drought waves induced by wintertime Mediterranean rainfall deficit. Geophysical Research Letters, 34, L07711.

Vautard, R., J. Cattiaux, P. Yiou, J.-N. Thepaut, and P. Ciais, 2010: Northern Hemisphere atmospheric stilling partly attributed to an increase in surface roughness. Nature Geoscience, 3(11), 756-761.

Vecchi, G.A. and T.R. Knutson, 2008: On estimates of historical North Atlantic tropical cyclone activity. Journal of Climate, 21(14), 3580-3600.

Vecchi, G.A. and B.J. Soden, 2007a: Effect of remote sea surface temperature change on tropical cyclone potential intensity. Nature, 450(7172), 1066-1070.

Vecchi, G.A. and B.J. Soden, 2007b: Global warming and the weakening of the tropical circulation. Journal of Climate, 20(17), 4316-4340.

Vecchi, G.A. and B.J. Soden, 2007c: Increased tropical Atlantic wind shear in model projections of global warming. Geophysical Research Letters, 34, L08702.

Vecchi, G.A., and A.T. Wittenberg, 2010: El Niño and our future climate: where do we stand? Climate Change, 1(2), 260-270.

Vecchi, G.A., B.J. Soden, A.T.Wittenberg, I.M. Held, A. Leetmaa, and M.J. Harrison, 2006: Weakening of tropical Pacific atmospheric circulation due to anthropogenic forcing. Nature, 441, 73-76.

Vecchi, G.A., K.L. Swanson, and B.J. Soden, 2008: Whither hurricane activity. Science, 322(5902), 687-689.

Velicogna, I., 2009: Increasing rates of ice mass loss from the Greenland and Antarctic ice sheets revealed by GRACE. Geophysical Research Letters, 36, L19503.

Vera, C. and G. Silvestri, 2009: Precipitation interannual variability in South America from the WCRP-CMIP3 multi-model dataset. Climate Dynamics, 32(7-8), 1003-1014

Vera, C., G. Silvestri, B. Liebmann, and P. Gonzalez, 2006: Climate change scenarios for seasonal precipitation in South America from IPCC-AR4 models. Geophysical Research Letters, 33, L13707.
Vermeer, M. and S. Rahmstorf, 2009: Global sea level linked to global temperature. Proceedings of the National Academy of Sciences, 106(51), 21527-21532.

Vicente-Serrano, S.M. and J.I. Lopez-Moreno, 2008: Nonstationary influence of the North Atlantic Oscillation on European precipitation. Journal of Geophysical Research-Atmospheres, 113, D20120.

Vicente-Serrano, S.M., S. Begueria, and J.I. Lopez-Moreno, 2010: A multiscalar drought index sensitive to global warming: The standardized precipitation evapotranspiration index. Journal of Climate, 23(7), 1696-1718.

Vidal, J.P., E. Martin, L. Franchisteguy, F. Habets, J.M. Soubeyroux, M. Blanchard, and M. Baillon, 2010: Multilevel and multiscale drought reanalysis over France with the Safran-Isba-Modcou hydrometeorological suite. Hydrology and Earth System Sciences, 14(3), 459-478.

Vilibic, I. and J. Sepic, 2010: Long-term variability and trends of sea level storminess and extremes in European Seas. Global and Planetary Change, 71(1-2), 1-12.

Villarini, G., F. Serinaldi, J.A. Smith, and W.F. Krajewski, 2009: On the stationarity of annual flood peaks in the continental United States during the 20th century. Water Resources Research, 45, W08417.

Vincent, C., E. Le Meur, D. Six, M. Funk, M. Hoelzle, and S. Preunkert, 2007: Very highelevation Mont Blanc glaciated areas not affected by the 20th century climate change. Journal of Geophysical Research - Atmospheres, 112, D09120.

Vincent, C., S. Auclair, and E.L.e. Meur, 2010: Outburst flood hazard for glacierdammed Lac de Rochemelon, France. Journal of Glaciology, 56(195), 91-100.

Vincent, L.A. and E. Mekis, 2006: Changes in daily and extreme temperature and precipitation indices for Canada over the 20th century. Atmosphere-Ocean, 44(2), 177-193

Vincent, L.A., X. Zhang, B.R. Bonsal, and W.D. Hogg, 2002: Homogenization of daily temperatures over Canada. Journal of Climate, 15(11), 1322-1334.

Vincent, L.A., T.C. Peterson, V.R. Barros, M.B. Marino, M. Rusticucci, G. Carrasco, E. Ramirez, L.M. Alves, T. Ambrizzi, M.A. Berlato, A.M. Grimm, J.A. Marengo, J. Molion, D.F. Moncunill, E. Rebello, Y.M.T. Anunciação, J. Quintana, J.L. Santos, J. Baez, G. Coronel, J. Garcia, I. Trebejo, M. Bidegain, M.R. Haylock, and D. Karoly, 2005: Observed trends in indices of daily temperature extremes in South America 1960-2000. Journal of Climate, 18(23), 5011-5023.

Vinchon, C., S. Aubie, Y. Balouin, L. Closset, M. Garcin, D. Idier, and C. Mallet, 2009: Anticipate response of climate change on coastal risks at regional scale in Aquitaine and Languedoc Roussillon (France). Ocean \& Coastal Management, 52(1), 47-56

Vinnikov, K.Y., A. Robock, N.A. Speranskaya, and A. Schlosser, 1996: Scales of temporal and spatial variability of midlatitude soil moisture. Journal of Geophysical Research - Atmospheres, 101(D3), 7163-7174.

Voldoire, A., and J.F. Royer, 2004: Tropical deforestation and climate variability. Climate Dynamics, 22(8), 857-874.

Vose, R.S., D.R. Easterling, and B. Gleason, 2005: Maximum and minimum temperature trends for the globe: an update through 2004. Geophysical Research Letters, 32, L23822.

Vrac, M., M. Stein, and K. Hayhoe, 2007: Statistical downscaling of precipitation through nonhomogeneous stochastic weather typing. Climate Research, 34(3), 169-184.

Vuichard, D. and M. Zimmermann, 1987: The 1985 catastrophic drainage of a moraine-dammed lake, Khumbu Himal, Nepal: cause and consequences. Mountain Research and Development, 7(2), 91-110.

Wakazuki, Y., M. Nakamura, S. Kanada, and C. Muroi, 2008: Climatological reproducibility evaluation and future climate projection of extreme precipitation events in the Baiu season using a high-resolution non-hydrostatic RCM in comparison with an AGCM. Journal of the Meteorological Society of Japan, 86(6), 951-967.

Walkden, M. and M. Dickson, 2008: Equilibrium erosion of soft rock shores with a shallow or absent beach under increased sea level rise. Marine Geology, 251(1-2), 75-84.

Walsh, J., 2005: Cryosphere and hydrology. In: Arctic Climate Impact Assessment. Cambridge Univesity Press, Cambridge, UK, pp. 183-242.

Wan, H., X.L. Wang, and V.R. Swail, 2010: Homogenization and trend analysis of Canadian near-surface wind speeds. Journal of Climate, 23(5), 1209-1225. 
Wang, A.H., T.J. Bohn, S.P. Mahanama, R.D. Koster, and D.P. Lettenmaier, 2009: Multimodel ensemble reconstruction of drought over the Continental United States. Journal of Climate, 22(10), 2694-2712.

Wang, B. and Q.H. Ding, 2006: Changes in global monsoon precipitation over the past 56 years. Geophysical Research Letters, 33, L06711.

Wang, B., Q.H. Ding, X.H. Fu, I.S. Kang, K. Jin, J. Shukla, and F. Doblas-Reyes, 2005: Fundamental challenge in simulation and prediction of summer monsoon rainfall. Geophysical Research Letters, 32, L15711.

Wang, G. and H.H. Hendon, 2007: Sensitivity of Australian rainfall to inter-El Niño variations. Journal of Climate, 20(16), 4211-4226.

Wang, G.L., 2005: Agricultural drought in a future climate: results from 15 global climate models participating in the IPCC 4th assessment. Climate Dynamics, 25(7-8), 739-753.

Wang, J. and X. Zhang, 2008: Downscaling and projection of winter extreme daily precipitation over North America. Journal of Climate, 21(5), 923-937.

Wang, S., R. McGrath, J. Hanafin, P. Lynch, T. Semmler, and P. Nolan, 2008: The impact of climate change on storm surges over Irish waters. Ocean Modelling, 25(1-2), 83-94

Wang, X., P. Zhai, and C. Wang, 2009: Variations in extratropical cyclone activity in northern East Asia. Advances in Atmospheric Sciences, 26(3), 471-479.

Wang, X.L., V.R. Swail, and F.W. Zwiers, 2006: Climatology and changes of extratropical cyclone activity: Comparison of ERA-40 with NCEP-NCAR reanalysis for 19582001. Journal of Climate, 19(13), 3145-3166.

Wang, X.L., V.R. Swail, F.W. Zwiers, X. Zhang, and Y. Feng, 2009a: Detection of external influence on trends of atmospheric storminess and northern oceans wave heights. Climate Dynamics, 32(2-3), 189-203.

Wang, X.L., F.W. Zwiers, V.R. Swail, and Y. Feng, 2009b: Trends and variability of storminess in the Northeast Atlantic region, 1874-2007. Climate Dynamics, 33(7-8), 1179-1195.

Wang, X.M., Y. Yang, Z.B. Dong, and C.X. Zhang, 2009: Responses of dune activity and desertification in China to global warming in the twenty-first century. Global and Planetary Change, 67(3-4), 167-185.

Wang, Y.Q., L.R. Leung, J.L. McGregor, D.K. Lee, W.C. Wang, Y.H. Ding, and F. Kimura, 2004: Regional climate modeling: Progress, challenges, and prospects. Journal of the Meteorological Society of Japan, 82(6), 1599-1628.

Wanner, H., J. Beer, J. Butikofer, T.J. Crowley, U. Cubasch, J. Fluckiger, H. Goosse, M. Grosjean, F. Joos, J.O. Kaplan, M. Kuttel, S.A. Muller, I.C. Prentice, O. Solomina, T.F. Stocker, P. Tarasov, M. Wagner, and M. Widmann, 2008: Mid- to Late Holocene climate change: an overview. Quaternary Science Reviews, 27(19-20), 1791-1828.

Watterson, I.G., 2006: The intensity of precipitation during extratropical cyclones in global warming simulations: a link to cyclone intensity? Tellus Series $A$ Dynamic Meteorology and Oceanography, 58(1), 82-97.

Webb, A.P. and P. Kench, 2010: The dynamic response of reef islands to sea level rise: Evidence from multi-decadal analysis of island change in the central Pacific. Global and Planetary Change, 72(3), 234-246.

Webster, P.J., 2008: Myanmar's deadly daffodil. Nature Geoscience, 1, 488-490.

Webster, P.J., G.J. Holland, J.A. Curry, and H.R. Chang, 2005: Changes in tropical cyclone number, duration, and intensity in a warming environment. Science, 309(5742), 1844-1846.

Wehner, M.F., G. Bala, P. Duffy, A.A. Mirin, and R. Romano, 2010: Towards direct simulation of future tropical cyclone statistics in a high-resolution global atmospheric model. Advances in Meteorology, 2010, Article 915303.

Weisse, R. and H. Günther, 2007: Wave climate and long-term changes for the Southern North Sea obtained from a high-resolution hindcast 1958-2002. Ocean Dynamics, 57(3), 161-172.

Weisse, R., H. Von Storch, and F. Feser, 2005: Northeast Atlantic and North Sea storminess as simulated by a regional climate model during 1958-2001 and comparison with observations. Journal of Climate, 18(3), 465-479.

Wells, N., S. Goddard, and M.J. Hayes, 2004: A self-calibrating Palmer Drought Severity Index. Journal of Climate, 17(12), 2335-2351.

Weng, H., S.K. Behera, and T. Yamagata, 2009: Anomalous winter climate conditions in the Pacific Rim during recent El Niño Modoki and El Niño events. Climate Dynamics, 32(5), 663-674.
Wernli, H., S. Dirren, M.A. Liniger, and M. Zillig, 2002: Dynamical aspects of the life cycle of the winter storm 'Lothar' (24-26 December 1999). Quarterly Journal of the Royal Meteorological Society, 128(580), 405-429.

Wettstein, J.J., and J.M. Wallace, 2010: Observed patterns of month-to-month stormtrack variability and their relationship to the background flow. Journal of the Atmospheric Sciences, 67(5), 1420-1437.

Whitney, F.A., H.J. Freeland, and M. Robert, 2007: Persistently declining oxygen levels in the interior waters of the eastern subarctic Pacific. Progress in Oceanography, 75(2), 179-199.

Wieczorek, G.F., T. Glade, M. Jakob, and O. Hungr, 2005: Climatic factors influencing occurrence of debris flows. In: Debris-Flow Hazards and Related Phenomena [Jakob, M. and O. Hungr (eds.)]. Springer, Berlin, Germany, pp. 325-362.

Wiggs, G.F.S., A.J. Baird, and R.J. Atherton, 2004: The dynamic effects of moisture on the entrainment and transport of sand by wind. Geomorphology, 59(1-4), 13-30.

Wilby, R.L., K.J. Beven, and N.S. Reynard, 2008: Climate change and fluvial flood risk in the UK: more of the same? Hydrological Processes, 22(14), 2511-2523.

Willett, K.M., N.P. Gillett, P.D. Jones, and P.W. Thorne, 2007: Attribution of observed surface humidity changes to human influence. Nature, 449(7163), 710-712.

Willett, K.M., P.D. Jones, N.P. Gillett, and P.W. Thorne, 2008: Recent changes in surface humidity: Development of the HadCRUH dataset. Journal of Climate, 21(20), 5364-5383.

Willett, K.M., P.D. Jones, P.W. Thorne, and N.P. Gillett, 2010: A comparison of large scale changes in surface humidity over land in observations and CMIP3 general circulation models. Environmental Research Letters, 5(2), 025210.

Wilson, P., J. McGourty, and M.D. Bateman, 2004: Mid- to late-Holocene coastal dune event stratigraphy for the north coast of Northern Ireland. Holocene, 14(3) 406-416.

Wing, A.A., A.H. Sobel, and S.J. Camargo, 2007: Relationship between the potential and actual intensities of tropical cyclones on interannual time scales. Geophysical Research Letters, 34, L08810.

Wittenberg, A.T., 2009: Are historical records sufficient to constrain ENSO simulations? Geophysical Research Letters, 36, L12702.

Wong, S., A.E. Dessler, N.M. Mahowald, P.R. Colarco, and A. da Silva, 2008: Long-term variability in Saharan dust transport and its link to North Atlantic sea surface temperature. Geophysical Research Letters, 35, L07812.

Woodroffe, C.D., 2008: Reef-island topography and the vulnerability of atolls to sea-level rise. Global and Planetary Change, 62(1-2), 77-96.

Woodruff, J.D., J.P. Donnelly, K.A. Emanuel, and P. Lane, 2008a: Assessing sedimentary records of paleohurricane activity using modeled hurricane climatology. Geochemistry Geophysics Geosystems, 9, Q09V10.

Woodruff, J.D., J.P. Donnelly, D. Mohrig, and W.R. Geyer, 2008b: Reconstructing relative flooding intensities responsible for hurricane-induced deposits from Laguna Playa Grande, Vieques, Puerto Rico. Geology, 36(5), 391-394.

Woodworth, P.L. and D.L. Blackman, 2004: Evidence for systematic changes in extreme high waters since the mid-1970s. Journal of Climate, 17(6), 1190-1197.

Woodworth, P.L., M. Menendez, and W.R. Gehrels, 2011: Evidence for centurytimescale acceleration in mean sea levels and for recent changes in extreme sea levels. Surveys in Geophysics, 32(4-5), 603-618, doi: 10.1007/s1071210011-19112-10718.

Woollings, T.J., B.J. Hoskins, M. Blackburn, and P. Berrisford, 2008: A new Rossby wave-breaking interpretation of the North Atlantic Oscillation. Journal of Atmospheric Science, 65(2), 609-626.

Woollings, T.J., A. Hannachi, B. Hoskins, and A. Turner, 2010: A regime view of the North Atlantic Oscillation and its response to anthropogenic forcing. Journal of Climate, 23(6), 1291-1307.

Wu, Q., and T. Zhang, 2010: Changes in active layer thickness over the QinghaiTibetan Plateau from 1995-2007. Journal of Geophysical Research, 115, D09107.

Wu, Y., M. Ting, R. Seager, H.-P. Huang, and M. Cane, 2011: Changes in storm tracks and energy transports in a warmer climate simulated by the GFDL CM2.1 model. Climate Dynamics, 37(1-2), 53-72.

Xie, B., Q. Zhang, and Y. Wang, 2008: Trends in hail in China during 1960-2005. Geophysical Research Letters, 35, L13801. 
Xie, S.-P., C. Deser, G.A. Vecchi, J. Ma, H. Teng, and A.T. Wittenberg, 2010: Global warming pattern formation: Sea surface temperature and rainfall. Journal of Climate, 23(4), 966-986.

Xin, W., L. Shiyin, G. Wanqin, and X. Junli, 2008: Assessment and simulation of glacier lake outburst floods for Longbasaba and Pida Lakes, China. Mountain Research and Development, 28(3), 310-317.

Xoplaki, E., J.F. Gonzalez-Rouco, J. Luterbacher, and H. Wanner, 2003: Mediterranean summer air temperature variability and its connection to the large-scale atmospheric circulation and SSTs. Climate Dynamics, 20(7-8), 723-739.

Xu, M., C.P. Chang, C.B. Fu, Y. Qi, A. Robock, D. Robinson, and H.M. Zhang, 2006: Steady decline of east Asian monsoon winds, 1969-2000: Evidence from direct ground measurements of wind speed. Journal of Geophysical Research Atmospheres, 111, D24111.

Yao, C., S. Yang, W. Qian, Z. Lin, and M. Wen, 2008: Regional summer precipitation events in Asia and their changes in the past decades. Journal of Geophysical Research-Atmospheres, 113, D17107.

Yeh, S.W., J.S. Kug, B. Dewitte, M.H. Kwon, B.P. Kirtman, and F.F. Jin, 2009: El Niño in a changing climate. Nature, 461, 511-514.

Yin, J., S.M. Griffies, and R.J. Stouffer, 2010: Spatial variability of sea level rise in twenty-first century projections. Journal of Climate, 23(17), 4585-4607.

Yiou, P., P. Ribereau, P. Naveau, M. Nogaj, and R. Brazdil, 2006: Statistical analysis of floods in Bohemia (Czech Republic) since 1825. Hydrological Sciences Journal, 51(5), 930-945.

Yoshimura, J., M. Sugi, and A. Noda, 2006: Influence of greenhouse warming on tropical cyclone frequency. Journal of the Meteorological Society of Japan, 84(2), 405-428.

You, Q., S. Kang, E. Aguilar, and Y. Yan, 2008: Changes in daily climate extremes in the eastern and central Tibetan Plateau during 1961-2005. Journal of Geophysical Research - Atmospheres, 113, D07101.

You, Q., S. Kang, E. Aguilar, N. Pepin, W.A. Flügel, Y. Yan, Y. Xu, Y. Zhang, and J. Huang, 2011: Changes in daily climate extremes in China and their connection to the large scale atmospheric circulation during 1961-2003. Climate Dynamics, 36(11-12), 2399-2417.

Young, I.R., S. Zieger, and A.V. Babanin, 2011: Global trends in wind speed and wave height. Science, 332(6028), 451-455.

Yu, J.Y. and H.Y. Kao, 2007: Decadal changes of ENSO persistence barrier in SST and ocean heat content indices: 1958-2001. Journal of Geophysical Research Atmospheres, 112, D13106.

Yu, K.F., J.X. Zhao, Q. Shi, and Q.S. Meng, 2009: Reconstruction of storm/tsunami records over the last 4000 years using transported coral blocks and lagoon sediments in the southern South China Sea. Quaternary International, 195(1-2), 128-137.

Zahn, M. and H. von Storch, 2010: Decreased frequency of North Atlantic polar lows associated with future climate warming. Nature, 467(7313), 309-312.

Zemp, M., W. Haeberli, M. Hoelzle, and F. Paul, 2006: Alpine glaciers to disappear within decades? Geophysical Research Letters, 33, L13504.

Zemp, M., W. Haeberli, and J. Eamer, 2007: Glaciers and ice caps. In: Global Outlook for Ice and Snow. UNEP/GRID, Arendal, Norway, pp. 115-152.

Zhai, P.M., X. Zhang, H. Wan, and X.H. Pan, 2005: Trends in total precipitation and frequency of daily precipitation extremes over China. Journal of Climate, 18(7), 1096-1108.

Zhang, K.Q., B.C. Douglas, and S.P. Leatherman, 2004: Global warming and coastal erosion. Climatic Change, 64(1-2), 41-58.

Zhang, Q., Y. Guan, and H. Yang, 2008: ENSO amplitude change in observation and coupled models. Advances in Atmospheric Sciences, 25(3), 361-366.

Zhang, R. and T.L. Delworth, 2009: A new method for attributing climate variations over the Atlantic Hurricane Basin's main development region. Geophysical Research Letters, 36, L06701.

Zhang, T., 2005: Influence of the seasonal snow cover on the ground thermal regime: An overview. Reviews of Geophysics, 43, RG4002.

Zhang, T., R.G. Barry, K. Knowles, J.A. Heginbottom, and J. Brown, 1999: Statistics and characteristics of permafrost and ground ice distribution in the Northern Hemisphere. Polar Geography, 23(2), 147-169.
Zhang, T., O.W. Frauenfeld, M.C. Serreze, A. Etringer, C. Oelke, J. McCreight, R.G. Barry, D. Gilichinsky, D. Yang, H. Ye, F. Ling, and S. Chudinova, 2005: Spatial and temporal variability of active layer thickness over the Russian Arctic drainage basin. Journal of Geophysical Research, 110, D16101.

Zhang, X., K.D. Harvey, W.D. Hogg, and T.R. Yuzyk, 2001: Trends in Canadian streamflow. Water Resources Research, 37(4), 987-998.

Zhang, X., F.W. Zwiers, and G. Li, 2004: Monte Carlo experiments on the detection of trends in extreme values. Journal of Climate, 17(10), 1945-1952.

Zhang, X., E. Aguilar, S. Sensoy, H. Melkonyan, U. Tagiyeva, N. Ahmed, N. Kutaladze, F. Rahimzadeh, A. Taghipour, T.H. Hantosh, P. Albert, M. Semawi, M.K. Ali, M.H.S. Al-Shabibi, Z. Al-Oulan, T. Zatari, I.A. Khelet, S. Hamoud, R. Sagir, M. Demircan, M. Eken, M. Adiguzel, L. Alexander, T.C. Peterson, and T. Wallis, 2005: Trends in Middle East climate extreme indices from 1950 to 2003. Journal of Geophysical Research - Atmospheres, 110, D22104.

Zhang, X., F.W. Zwiers, G.C. Hegerl, F.H. Lambert, N.P. Gillett, S. Solomon, P.A. Stott, and T. Nozawa, 2007: Detection of human influence on twentieth-century precipitation trends. Nature, 448(7152), 461-465.

Zhang, X., J. Wang, F.W. Zwiers, P. Ya, and P.Y. Groisman, 2010: The influence of large scale climate variability on winter maximum daily precipitation over North America. Journal of Climate, 23(11), 2902-2915.

Zhang, X.D., J.E. Walsh, J. Zhang, U.S. Bhatt, and M. Ikeda, 2004: Climatology and interannual variability of arctic cyclone activity: 1948-2002. Journal of Climate, 17(12), 2300-2317.

Zhang, X.D., A. Sorteberg, J. Zhang, R. Gerdes, and J.C. Comiso, 2008: Recent radical shifts of atmospheric circulations and rapid changes in Arctic climate system. Geophysical Research Letters, 35, L22701.

Zhang, X.Y., S.L. Gong, T.L. Zhao, R. Arimoto, Y.Q. Wang, and Z.J. Zhou, 2003: Sources of Asian dust and role of climate change versus desertification in Asian dust emission. Geophysical Research Letters, 30(24), 2272.

Zhang, Y.Q., C.M. Liu, Y.H. Tang, and Y.H. Yang, 2007: Trends in pan evaporation and reference and actual evapotranspiration across the Tibetan Plateau. Journal of Geophysical Research - Atmospheres, 112, D12110.

Zhao, C.S., X. Dabu, and Y. Li, 2004: Relationship between climatic factors and dust storm frequency in Inner Mongolia of China. Geophysical Research Letters, 31, L01103.

Zhao, L., Q. Wu, S.S. Marchenko, and N. Sharkhuu, 2010: Thermal state of permafrost and active layer in Central Asia during the international polar year. Permafrost and Periglacial Processes, 21(2), 198-207.

Zhao, M., I.M. Held, S.J. Lin, and G.A. Vecchi, 2009: Simulations of global hurricane climatology, interannual variability, and response to global warming using a 50-km resolution GCM. Journal of Climate, 22(24), 6653-6678.

Zhong, D.C., 1999: The dynamic changes and trends of modern desert in China. Advance in Earth Sciences, 14(3), 229-234 (in Chinese).

Zhou, T.J., L.X. Zhang, and H.M. Li, 2008a: Changes in global land monsoon area and total rainfall accumulation over the last half century. Geophysical Research Letters, 35, L16707.

Zhou, T.J., R.C. Yu, H.M. Li, and B. Wang, 2008b: Ocean forcing to changes in global monsoon precipitation over the recent half-century. Journal of Climate, 21(15), 3833-3852.

Zhou, Z.J. and G.C. Zhang, 2003: Typical severe dust storms in northern China during 1954-2002. Chinese Science Bulletin, 48(21), 2366-2370.

Zimmermann, M., P. Mani, and H. Romang, 1997: Magnitude-frequency aspects of alpine debris flows. Eclogae Geologicae Helvetiae, 90(3), 415-420.

Zin, W.Z.W., S. Jamaludin, S.M. Deni, and A.A. Jemain, 2009: Recent changes in extreme rainfall events in Peninsular Malaysia: 1971-2005. Theoretical and Applied Climatology, 99(3-4), 303-314.

Zolina, O., C. Simmer, A. Kapala, S. Bachner, S. Gulev, and H. Maechel, 2008: Seasonally dependent changes of precipitation extremes over Germany since 1950 from a very dense observational network. Journal of Geophysical Research - Atmospheres, 113, D06110.

Zolina, 0., C. Simmer, K. Belyaev, A. Kapala, and S. Gulev, 2009: Improving estimates of heavy and extreme precipitation using daily records from European rain gauges. Journal of Hydrometeorology, 10(3), 701-716. 
Zolina, 0., C. Simmer, S.K. Gulev, and S. Kollet, 2010: Changing structure of European precipitation: Longer wet periods leading to more abundant rainfalls. Geophysical Research Letters, 37, L06704.

Zorita, E., T.F. Stocker, and H. von Storch, 2008: How unusual is the recent series of warm years? Geophysical Research Letters, 35, L24706.
Zou, X., L.V. Alexander, D. Parker, and J. Caesar, 2006: Variations in severe storms over China. Geophysical Research Letters, 33, L17701.

Zwiers, F.W., X. Zhang, and Y. Feng, 2011: Anthropogenic influence on long return period daily temperature extremes at regional scales. Journal of Climate, 24(3), 881-892. 